\title{
Pathogen-induced cell wall remodeling and production of Danger Associated Molecular Patterns (DAMPs)
}

\author{
Dissertation \\ for the award of the degree \\ "Doctor rerum naturalium" \\ of the University of Goettingen \\ within the doctoral program \\ International Research Training Group 2172 - PRoTECT \\ of the Georg-August University School of Science (GAUSS) \\ submitted by \\ Sina Barghahn \\ from Oldenburg (Oldb), Germany
}

Göttingen 2020 



\section{Thesis Committee}

Prof. Dr. Volker Lipka

Department of Plant Cell Biology, Albrecht-von-Haller Institute for Plant Science, University of Goettingen

PD Dr. Till Ischebeck

Department of Plant Biochemistry, Albrecht-von-Haller Institute for Plant Science, University of Goettingen

Prof. Dr. Harry Brumer

Department of Chemistry, Michael Smith Laboratories, University of British Columbia

\section{Members of the Examination Board}

Referee: $\quad$ Prof. Dr. Volker Lipka

Department of Plant Cell Biology, Albrecht-von-Haller Institute for Plant Science, University of Goettingen

$2^{\text {nd }}$ Referee: PD Dr. Till Ischebeck

Department of Plant Biochemistry, Albrecht-von-Haller Institute for Plant Science, University of Goettingen

\section{Further members of the Examination Board}

Prof. Dr. Harry Brumer

Department of Chemistry, Michael Smith Laboratories, University of British Columbia

Prof. Dr. Ivo Feußner

Department of Plant Biochemistry, Albrecht-von-Haller Institute for Plant Science, University of Goettingen

Prof. Dr. Gerhard Braus

Department of Molecular Microbiology and Genetics, Institute of Microbiology and Genetics, University of Goettingen

\section{Dr. Marcel Wiermer}

Department of Molecular Biology of Plant-Microbe Interactions, Albrecht-von-Haller Institute for Plant Science, University of Goettingen 



\section{Promovierenden-Erklärung \\ der Georg-August-Universität Göttingen}

Die Gelegenheit zum vorliegenden Promotionsvorhaben ist mir nicht kommerziell vermittelt worden. Insbesondere habe ich keine Organisation eingeschaltet, die gegen Entgelt Betreuerinnen und Betreuer für die Anfertigung von Dissertationen sucht oder die mir obliegenden Pflichten hinsichtlich der Prüfungsleistungen für mich ganz oder teilweise erledigt.

Hilfe Dritter wurde bis jetzt und wird auch künftig nur in wissenschaftlich vertretbarem und prüfungsrechtlich zulässigem Ausmaß in Anspruch genommen. Insbesondere werden alle Teile der Dissertation selbst angefertigt; unzulässige fremde Hilfe habe ich dazu weder unentgeltlich noch entgeltlich entgegengenommen und werde dies auch zukünftig so halten.

Die Ordnung zur Sicherung der guten wissenschaftlichen Praxis an der Universität Göttingen wird von mir beachtet.

Eine entsprechende Promotion wurde an keiner anderen Hochschule im In- oder Ausland beantragt; die eingereichte Dissertation oder Teile von ihr wurden/werden nicht für ein anderes Promotionsvorhaben verwendet.

Mir ist bekannt, dass unrichtige Angaben die Zulassung zur Promotion ausschließen bzw. später zum Verfahrensabbruch oder zur Rücknahme des erlangten Grades führen können. 



\section{Abstract}

For a plant pathogen, overcoming the plant cell wall is crucial for a successful infection. Thus, pathogens evolved different strategies to invade their host plants. These include entry through natural openings such as stomata and wounds or direct penetration of plant cell walls with specialised invasion structures that generate high pressure as well as cell wall degrading enzymes (CWDEs). CWDEs can be classified into different groups according to their potential function and include e.g. Glycoside Hydrolases (GHs), which are implicated in the hydrolysis of glycosidic linkages in complex carbohydrates such as the plant cell wall component cellulose. GHs have been shown to be involved in pathogenicity of hemibiotrophic and necrotophic plant-pathogenic fungi. However, the role of GHs in biotrophic plant-pathogenic fungi has not been elucidated so far.

The first part of the present study focused on the identification and functional characterization of GH17 family members of the powdery mildew Blumeria graminis f.sp. hordei (Bgh) that may contribute to pathogenicity due to a transcriptional induction during infection of immunocompromised Arabidopsis plants. Of these, Bgh GH17 protein BGH06777 was successfully expressed in the heterologous system $P$. pastoris, purified and functionally characterized. The glycosylated enzyme showed optimal activity at $\mathrm{pH} 5.5$ in a temperature range from $25^{\circ} \mathrm{C}-51^{\circ} \mathrm{C}$ and hydrolysed $\beta-1,3$-glucans with a minimum length of four glucose residues. The catalytic efficiencies for hydrolysis of the $\beta$-1,3-glucan hexamer and pentamer were $1.858 \mathrm{mM}^{-1} \mathrm{~s}^{-1}$ and $0.3836 \mathrm{mM}^{-1} \mathrm{~s}^{-1}$, respectively. ${ }^{18} \mathrm{O}$ labelling of the products revealed that the enzyme contains at least six substrate binding sites comprised of four negative and two positive subsites. In conclusion, the detailed biochemical characterization conducted in this study suggests that BGH06777 might degrade $\beta$-1,3-glucans present in plant papillae, however, the exact function and localization of this protein remains to be shown.

Plants are able to perceive potential pathogens through the recognition of conserved non-self microbial structures, so-called pathogen or microbe-associated molecular patterns (PAMPs/MAMPs), at the plant surface via pattern recognition receptors (PRRs). Furthermore, plants can detect self-molecules that are only abundant upon cell damage or wounding, which are called damage or danger-associated molecular patterns (DAMPs). Both, MAMP or DAMP recognition triggers a signaling cascade that leads to the induction of defence responses. It is conceivable to postulate that the activity of CWDEs results in the release of cell-wall derived oligosaccharides with DAMP capacity. Thus, the second part of this study aimed at identifying novel cell-wall derived DAMPs and molecular components of the corresponding plant perception and signaling machinery. As a major result of this work, mixed linkage glucan (MLG) oligosaccharides were shown to trigger immune responses in the dicot model plant Arabidopsis and the monocot barley. The MLG-induced responses were similar to plant responses to the well-characterized MAMPs and DAMPs chitin, flg22 and OGs. In contrast to other MAMPs and DAMPs, MLG oligosaccharides did not elicit a detectable generation of reactive oxygen species or affect seedling growth in Arabidopsis. MLGs are abundant cell wall components of monocot grasses, e.g. barley, and the plant-pathogenic fungus Rhynchosporium commune (formerly $R$. secalis) but are absent in the dicot model plant Arabidopsis. Thus, MLG oligosaccharides might 
function in a plant-species specific manner as MAMP or DAMP (or both). A reverse genetic screen conducted with a collection of known DAMP/MAMP receptor and co-receptor mutants revealed that MLG perception and downstream signaling is likely to involve so far unknown molecular components. 


\section{Zusammenfassung}

Für ein Pflanzenpathogen ist das Überwinden der Zellwand entscheidend für eine erfolgreiche Infektion. Daher haben Pflanzenpathogene verschiedene Strategien entwickelt, um in Pflanzen einzudringen. Diese Strategien schließen das Eindringen über natürlich vorkommende Öffnungen wie Stomata oder Verwundungen, direkte Penetration mit spezialisierten Strukturen, die einen hohen Druck erzeugen, sowie Zellwand hydrolysierende Enzyme (CWDEs) ${ }^{1}$ ein. Abhängig von ihrer potentiellen Funktion, können CWDEs in verschiedene Gruppen klassifiziert werden. Unter Anderem gibt es die Klasse der Glycosyl Hydrolasen (GHs), die glykosidische Bindungen in komplexen Karbohydraten spalten wie zum Beispiel Zellulose, einem Bestandteil der pflanzlichen Zellwand. Es wurde gezeigt, dass GHs wichtig für die Pathogenität von hemibiotrophen und necrotrophen Pflanzenpathogenen sind. Die Rolle von GHs in biotrophen Pflanzenpathogenen wurde hingegen noch nicht geklärt.

Der erste Teil dieser Arbeit konzentrierte sich auf die Identifizierung und Charakterisierung der Mitglieder der GH17 Familie des Mehltau Pilzes Blumeria graminis f.sp. hordei (Bgh). Diese Familie spielt möglicherweise eine Rolle in der Pathogenität, da die Transkription dieser Familie während der Infektion von immunsupprimierten Arabidopsis Pflanzen induziert ist. Das Bgh Protein BGH06777 konnte erfolgreich in dem heterologen Expressionssystem $P$. pastoris exprimiert und schließlich aufgereinigt und funktional charakterisiert werden. Das glykolisierte Protein zeigte die optimale Aktivität bei einem pH Wert von 5.5 in einem Temperaturbereich von $25^{\circ} \mathrm{C}$ bis $51^{\circ} \mathrm{C}$ und hydrolisierte $\beta$-1,3-glukane mit einer minimalen Länge von vier Glukosemolekülen. Die katalytischen Effizienzen für die Hydrolyse des $\beta$-1,3-glukan Hexamers und $\beta$-1,3-Glukan Pentamers lagen bei jeweils $1.858 \mathrm{mM}^{-1} \mathrm{~s}^{-1}$ und $0.3836 \mathrm{mM}^{-1} \mathrm{~s}^{-1}$. Die Markierung der Hydrolyseprodukte mit ${ }^{18} \mathrm{O}$ ergab, dass das Enzym mindestens sechs Bindestellen für das Substrat hat bestehend aus vier negativen und zwei positiven Bindestellen. Die detallierte biochemische Analysis deutet an, dass BGH06777 $\beta$-1,3-glukane, die in pflanzlichen Papillen zu finden sind, hydrolysieren könnte. Die exakte Funktion sowie die Lokalisierung dieses Enzyms müssen jedoch noch gezeigt werden.

Pflanzen können potentielle Pathogene durch das Erkennen von konservierten mikrobiellen Struktuen, sogenannten Pathogen- oder Mikroben-assoziierten molekularen Mustern (PAMPs/MAMPs), an der Oberfläche durch membranständige Rezeptoren wahrnehmen. Weiterhin können Pflanzen auch Moleküle wahrnehmen, die von der Pflanze selbst stammen, aber nur nach Verwundung oder Beschädigung der Zelle vorhanden sind. Diese Moleküle werden Schaden- oder Gefahr-assoziierte molekulare Muster (DAMPs) genannt. Sowohl MAMPs als auch DAMPs aktivieren eine Signalkaskade, die zur Induktion der Immunantwort führt. Es ist vorstellbar, dass Zellwandfragmente mit DAMP Kapazität durch die Aktivität von CWDEs entstehen.

Der zweite Teil der Arbeit fokussiert sich daher auf die Identifizierung von neuen DAMP Molekülen, die von der Zellwand stammen, sowie den jeweiligen molekularen Komponenten, die an der Perzeption und der Signaltransduktion beteiligt sind. Eines der wichtigsten Ergebnisse dieser Arbeit

\footnotetext{
${ }^{1} \mathrm{Im}$ Folgenden werden für sämtliche Abkürzungen die englischen Abkürzungen verwendet (siehe auch: List of Abbreviations, V)
} 
ist, dass $\beta$-1,3;1,4-glukan Oligosaccharide Immunantworten in der dikotylen Pflanze Arabidopsis und der monokotylen Nutzpflanze Gerste auslösen. Diese Immunantworten ähneln den pflanzlichen Abwehrantworten, die durch die gut charakterisierten MAMPs und DAMPs Chitin, Flagellin und Oligogalakturonide ausgelöst werden. Im Gegensatz zu anderen MAMPs und DAMPs aktivieren die Oligosaccharide jedoch nicht die Generierung von reaktiven Sauerstoffspezies und beeinflussen auch nicht das Wachstum von Arabidopsis Setzlingen. Das $\beta-1,3 ; 1,4-$ glukan Polymer ist ein Bestandteil der Zellwand von monokotylen Gräsern, wie zum Beispiel Gerste, und dem Pflanzenpathogen Rhynchosporium commune (ehemals bekannt als $R$. secalis), aber ist kein Zellwandkomponent der dikotylen Modellpflanze Arabidopsis. Dies legt den Schluss nahe, dass $\beta-1,3 ; 1,4$-glucan Oligosaccharide in einer Pflanzenart-spezifischen Weise als MAMP oder DAMP (oder beidem) agieren. Ein revers genetischer Ansatz mit einer Kollektion von bekannten MAMP/DAMP Rezeptorund Co-Rezeptor Mutanten zeigte, dass die Perzeption sowie die Signaltransdkution wahrscheinlich bisher unbekannte molekulare Komponenten involviert. 


\section{List of Abbreviations}

$\begin{array}{ll}\alpha & \text { alpha/anti } \\ { }^{\circ} \mathrm{C} & \text { degree Celsius } \\ \mu \mathrm{g} & \text { microgramm } \\ \mu \mathrm{l} & \text { microliter } \\ \mu \mathrm{M} & \text { micromolar }\end{array}$

\section{A. fumigatus Aspergillus fumigatus}

AP alkaline phosphatase

APS ammonium persulfate

A. thaliana Arabidopsis thaliana

BAK1

BRI-1 ASSOCIATED KINASE 1

B. cinerea Botrytis cinerea

Bgh

Blumeria graminis f.sp. hordei

BIK1

BOTRYTIS INDUCED KINASE1

BMGY Buffered Glycerol-complex medium

BMMY Buffered Methanol-complex medium

BRI-1 brassinosteroid sensitive 1

BSA bovine serum albumin

B. subtilis Bacillus subtilis

$\begin{array}{ll}\mathrm{Ca}^{2+} & \text { Calcium } \\ \text { CBB } & \text { Coomassie Brilliant Blue } \\ \text { CBM } & \text { carbohydrate binding domain } \\ \text { CDNA } & \text { complementary DNA } \\ \text { CE } & \text { carbohydrate esterases } \\ \text { CEBiP } & \text { CHITIN ELICTOR BINDING PROTEIN } \\ \text { CERK1 } & \text { CHITIN ELICITOR RECEPTOR KINASE 1 } \\ \text { Col-0 } & \text { Columbia-0 } \\ \text { CSL } & \text { cellulose synthase like } \\ \text { CWDE } & \text { cell wall degrading enzyme } \\ & \\ \text { DAMP } & \text { danger/damage-associated molecular pattern } \\ \text { ddH } 20 & \text { double-distilled water } \\ \text { DNA } & \text { deoxyribonucleic acid } \\ \text { DNase } & \text { deoxyribonuclease } \\ \text { dNTP } & \text { desoxyribonucleotidetriphosphate } \\ \text { DTT } & \text { Dithiothreitol }\end{array}$




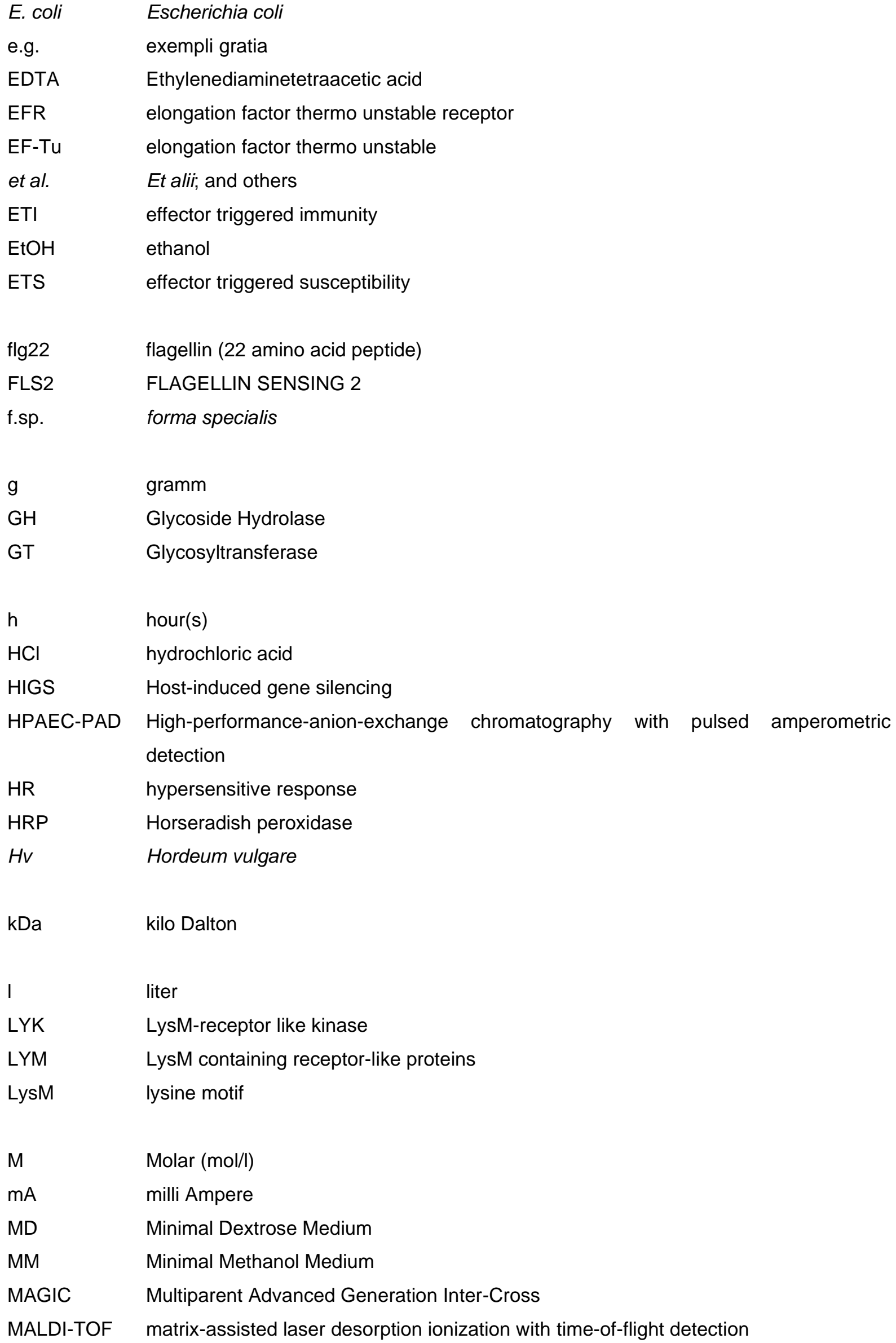




$\begin{array}{ll}\text { MAMP } & \text { microbe associated molecular pattern } \\ \text { MAPK } & \text { mitogen activated protein kinase } \\ \text { min } & \text { minute(s) } \\ \text { ml } & \text { milliliter } \\ \text { MLG } & \text { mixed linkage glucan } \\ \text { mM } & \text { millimolar } \\ \text { M. oryzae } & \text { Magnaporthe oryzae } \\ \text { MS } & \text { Murashige-Skoog } \\ \text { MTI } & \text { MAMP triggered immunity } \\ & \\ \text { OD } 600 & \text { Optical Density at a wavelength of } 600 \mathrm{~nm} \\ \text { OGs } & \text { oligogalacturonides } \\ \text { O. sativa } & \text { Oryza sativa } \\ \text { Os } & \text { Oryza sativa }\end{array}$

PCR Polymerase Chain Reaction

PEPR Pep receptor

Pep plant elicitor peptides

$\mathrm{pH} \quad$ negative decimal logarithm of the $\mathrm{H}^{+}$concentration

PIC Protease Inhibitor Cocktail

$\mathrm{PL} \quad$ polysaccharide lyase

P. pastoris Pichia pastoris

PRR Pattern recognition receptor

PTI PAMP-triggered immunity

PVDF polyveniylidene fluoride

\section{R. commune Rhynchosporium commune}

RLCK Receptor like cytoplasmic kinase

RLK receptor-like kinase

RLP receptor-like protein

RNA ribonucleic acid

ROS reactive oxygen species

rpm rounds per minute

RPM reads per million

RT room temperature

S second(s)

SCFE1 SCLEROTINIA CULTURE FILTRATE ELICITOR1

SDS Sodium dodecyl sulfate

SDS-PAGE Sodium dodecyl sulfate polyacrylamide gel electrophoresis 


$\begin{array}{ll}\text { SOBIR } & \text { SUPPRESSOR OF BIR1-1 } \\ \text { Taq } & \text { Thermus aquaticus } \\ \text { TAE } & \text { Tris-acetic acid EDTA } \\ \text { TBS-T } & \text { Tris buffered saline - Tween-20 } \\ \text { T-DNA } & \text { Transfer-DNA } \\ \text { TEMED } & \text { Tetramethylethylenediamine } \\ \text { TLC } & \text { Thin Layer Chromatography } \\ & \\ \text { U } & \text { Unit } \\ \text { V } & \\ \text { WAK1 } & \text { Volt } \\ \text { Ws } & \text { Wall-Associated kinase1 } \\ \text { YNB } & \text { Wassilewskija } \\ \text { YPD } & \text { Yeast Nitrogen Base } \\ & \text { Yeast Extract-Peptone-Dextrose }\end{array}$




\section{Table of contents}

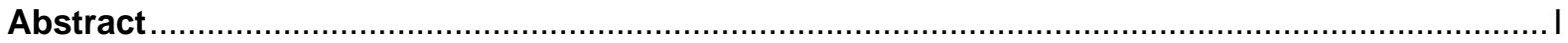

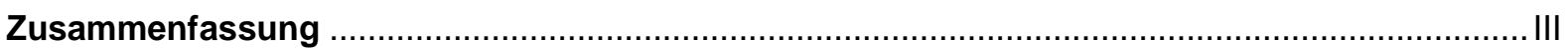

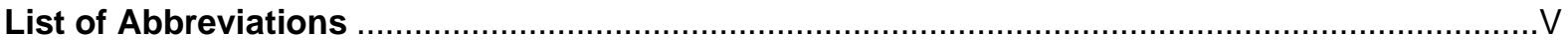

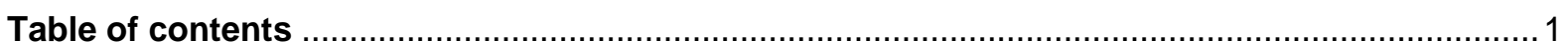

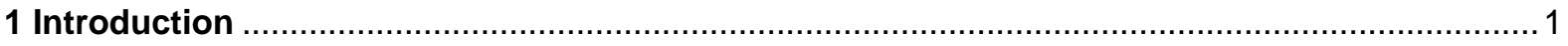

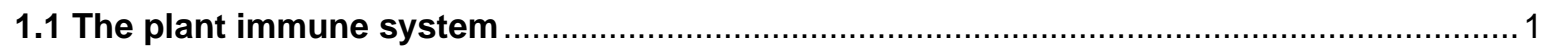

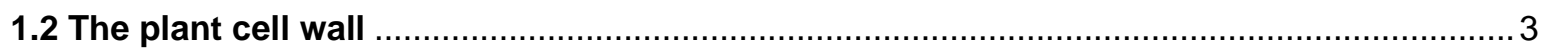

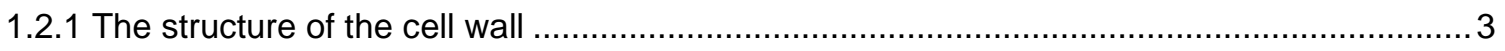

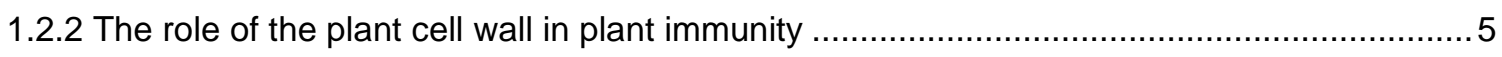

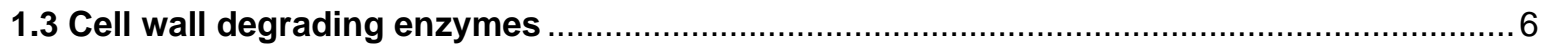

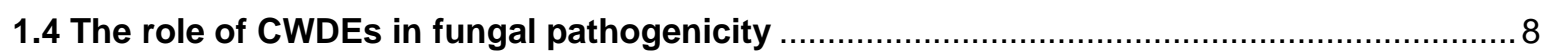

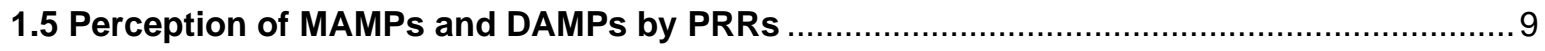

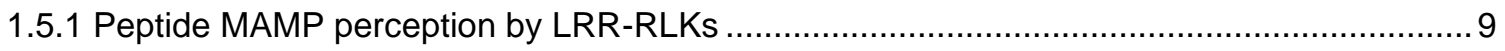

1.5.2 Perception of carbohydrate MAMPs by LysM domain containing RLKs ........................11

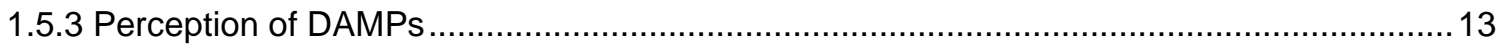

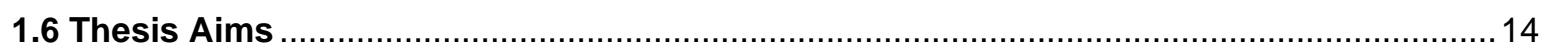

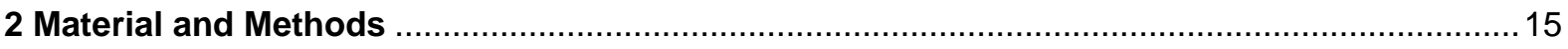

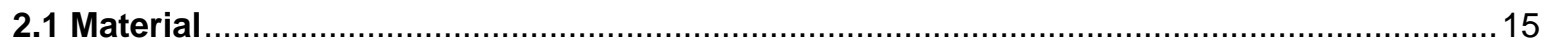

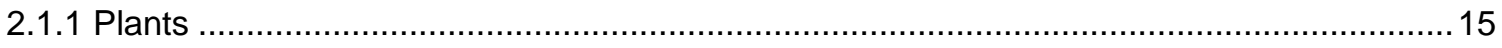

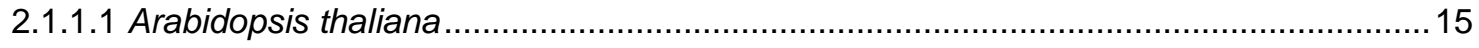

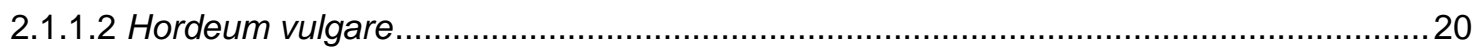

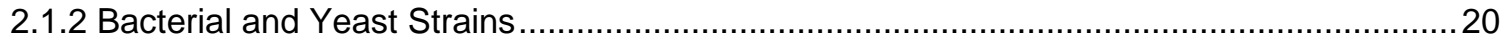

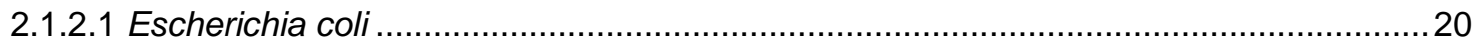

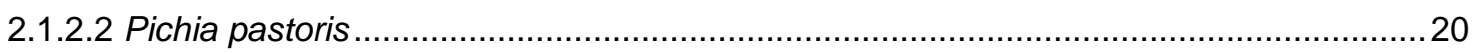

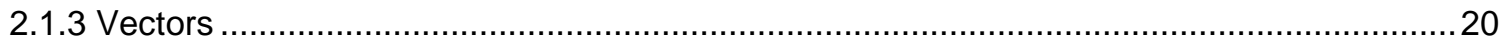

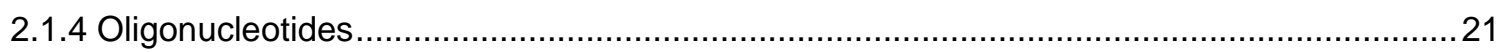




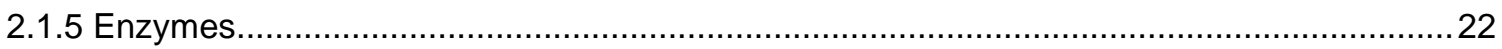

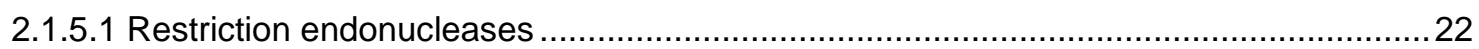

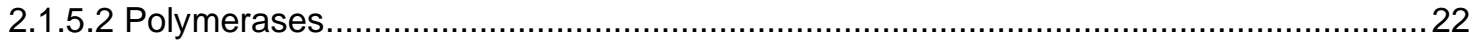

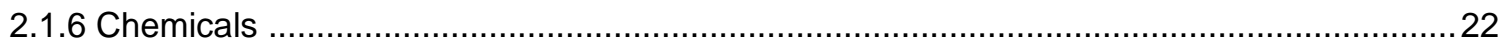

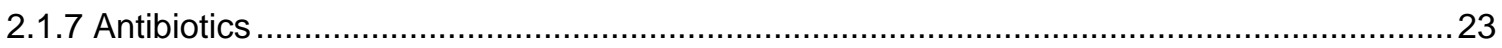

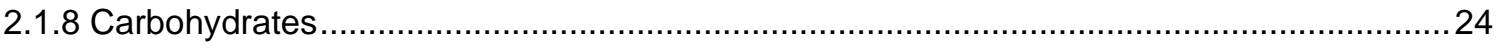

2.1.9 Media

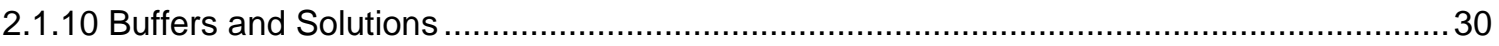

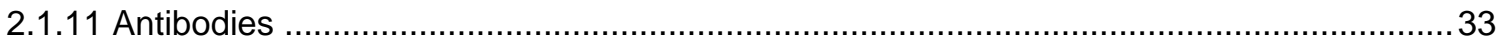

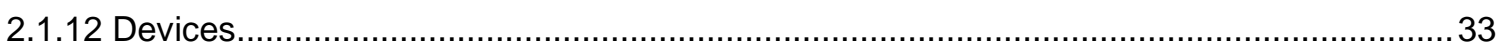

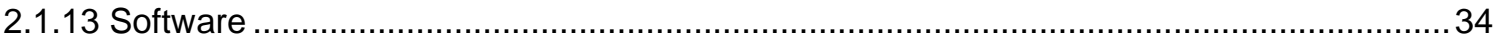

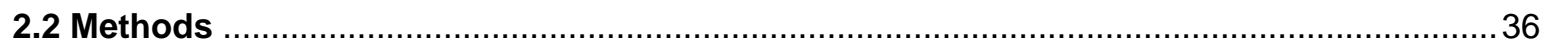

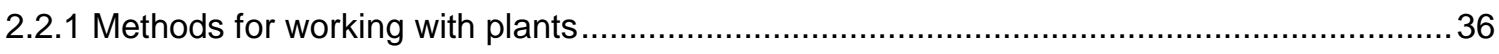

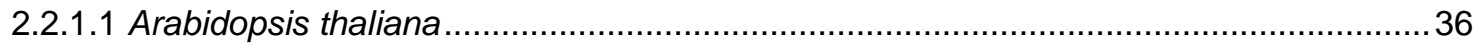

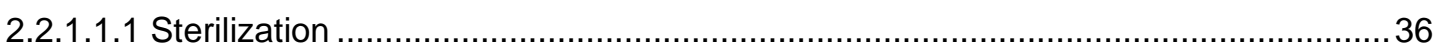

2.2.1.1.2 Plant growth conditions for growth on soil ......................................................... 36

2.2.1.1.3 Plant growth conditions for in-vitro culture ….................................................. 36

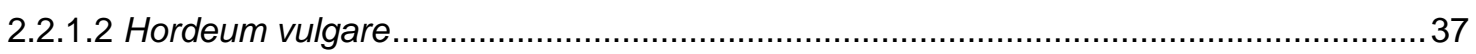

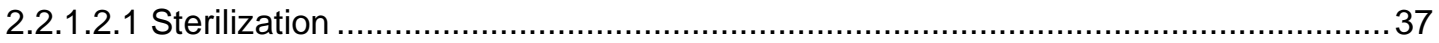

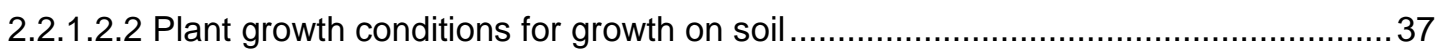

2.2.1.2.3 Treatment of $H$. vulgare for immunoblot analysis.............................................. 37

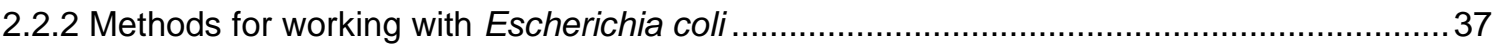

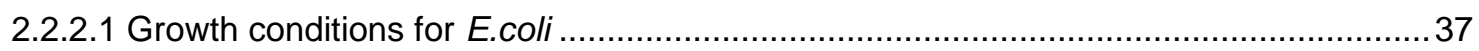

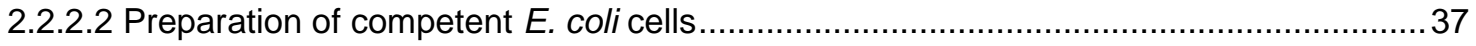

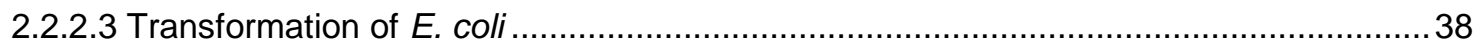

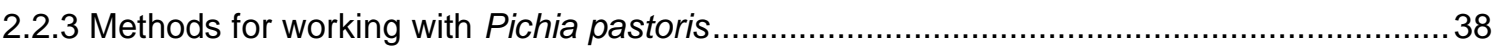

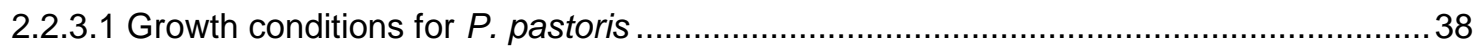

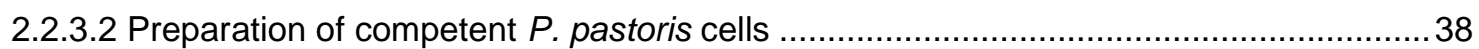

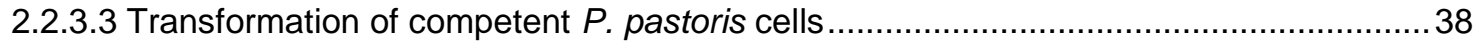

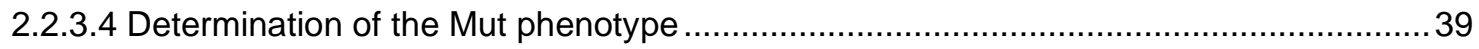

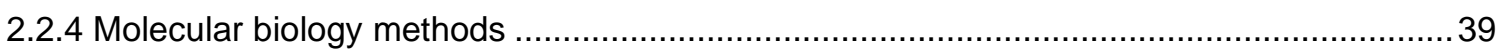


2.2.4.1 Isolation of genomic DNA of $A$. thaliana .

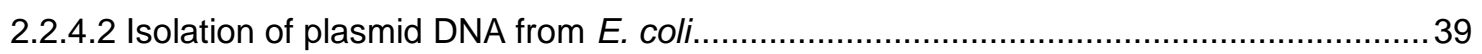

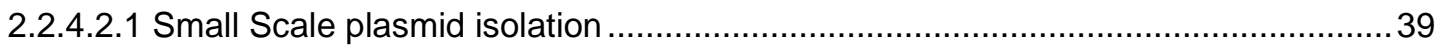

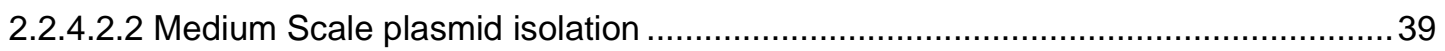

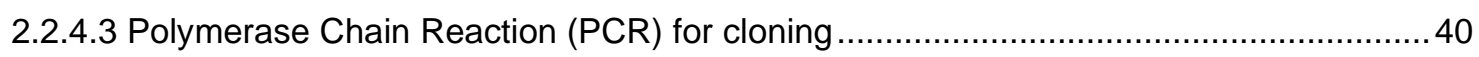

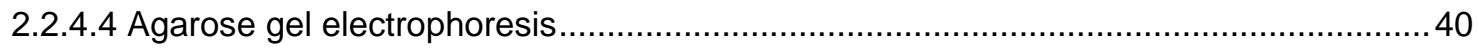

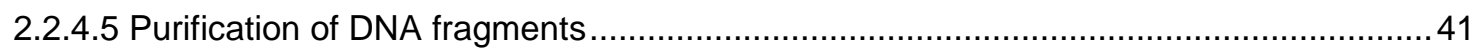

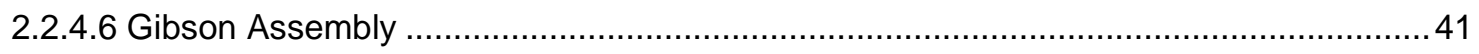

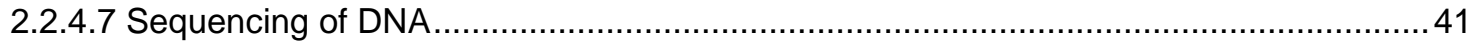

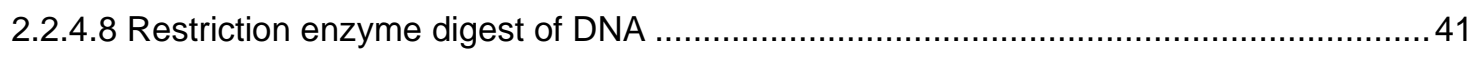

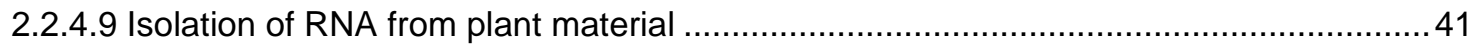

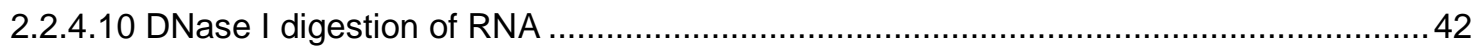

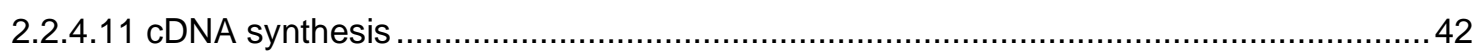

2.2.4.12 Quantitative reverse transcription PCR (qRT-PCR) ............................................. 42

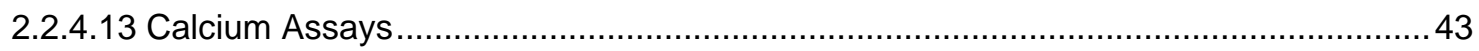

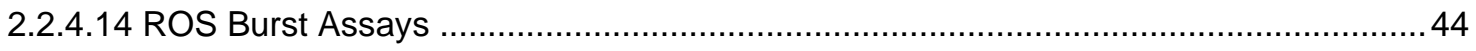

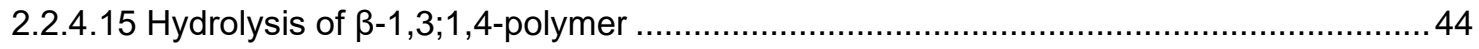

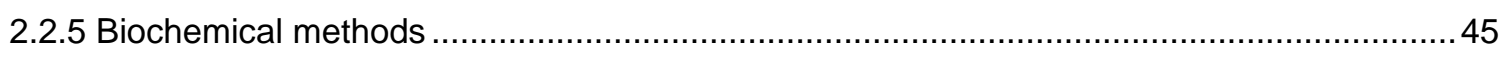

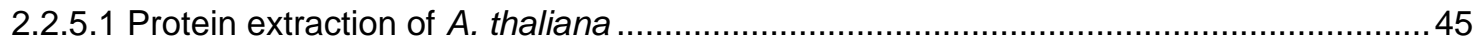

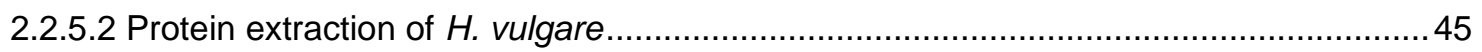

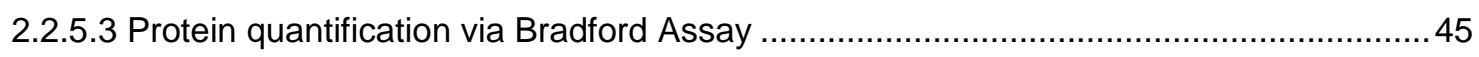

2.2.5.4 SDS-polyacrylamide gel electrophoresis (SDS-PAGE) …......................................... 46

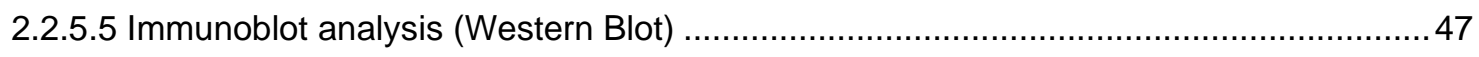

2.2.5.6 Coomassie Staining of PVDF membranes and SDS gels ....................................... 47

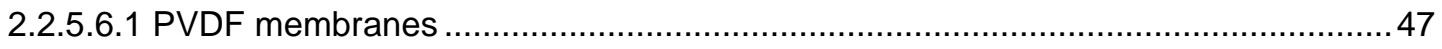

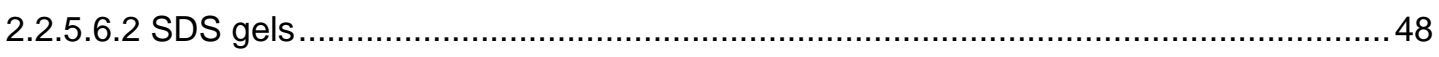

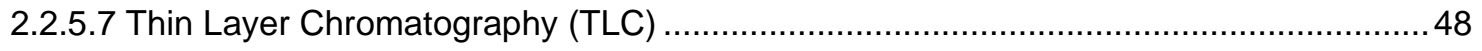

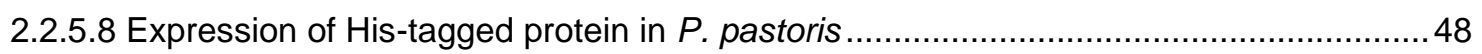

2.2.5.8.1 Small Scale Expression of secreted proteins in P. pastoris ................................... 48

2.2.5.8.2 Large Scale Expression of secreted proteins in P. pastoris................................... 49

2.2.5.9 Extraction and purification of His-tagged protein in P. pastoris .................................. 49 
2.2.5.10 Functional characterization of enzymes............................................................ 50

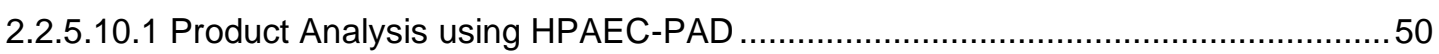

2.2.5.10.2 Confirmation of protein mass by mass-spectrometry ........................................ 50

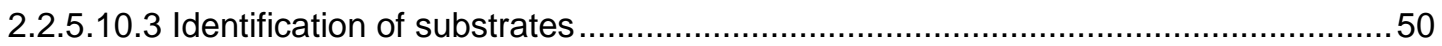

2.2.5.10.4 Identification of the temperature optimum ....................................................50

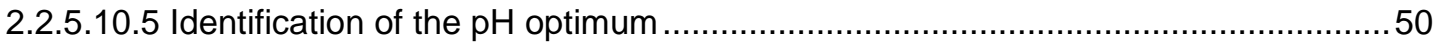

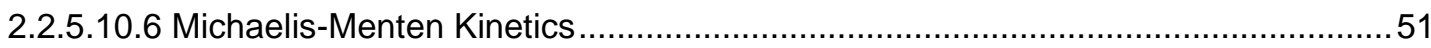

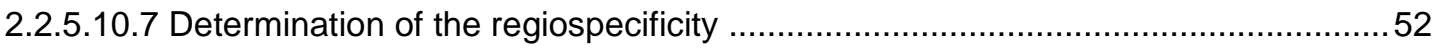

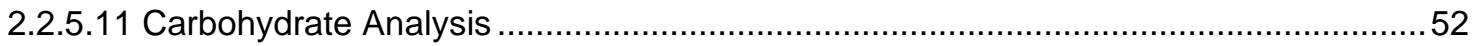

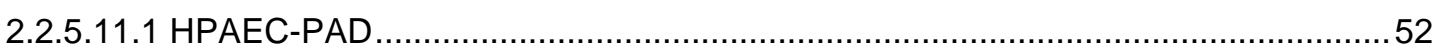

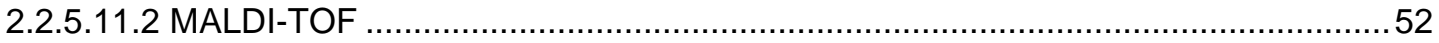

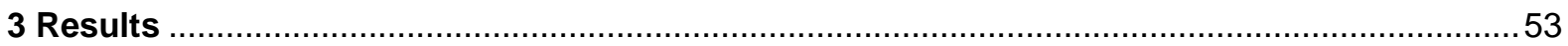

3.1 Identification and functional characterization of $\mathbf{B g h}$ CWDEs ........................................ 53

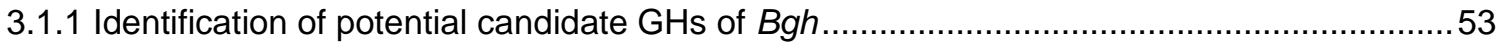

3.1.2 The family GH17 was chosen for further analysis ....................................................... 54

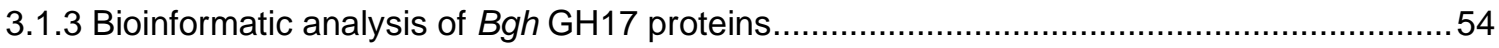

3.1.4 Analysis of the potential role of $\mathrm{Bgh} \mathrm{GH17}$ genes in pathogenicity .................................55

3.1.5 Recombinant production of Bgh GH17 proteins in Pichia pastoris ....................................56

3.1.6 $\beta$-1,3-glucan oligosaccharides are substrates of BGH06777 ........................................59

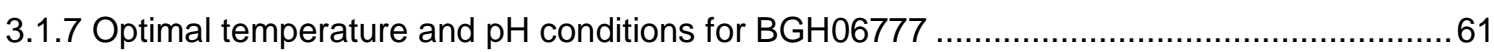

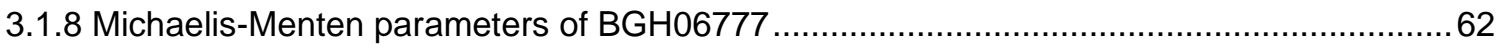

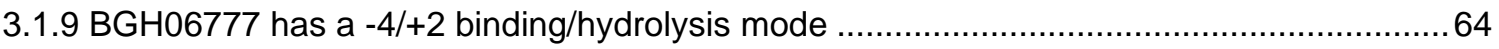

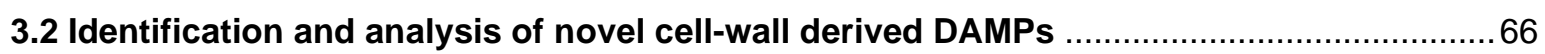

3.2.1 Screen to identify cell-wall derived DAMPs in Arabidopsis..............................................66

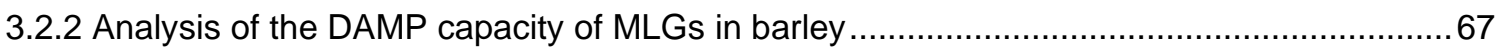

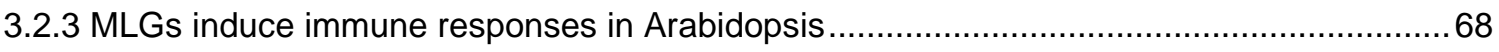

3.2.4 MLG oligosaccharides from a second company can induce immune responses in barley

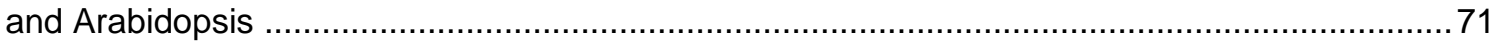

3.2.5 Commercially available MLGs do not contain major quantitative contaminants.................. 75

3.2.6 Hydrolysis products of the barley $\beta-1,3 ; 1,4$-glucan polymer induce defence responses in Arabidopsis 
3.2.7 Hydrolysis products of the barley $\beta-1,3 ; 1,4$-glucan polymer do not inhibit seedling growth 84

3.3 Molecular components involved in MLG perception could not be identified with reverse

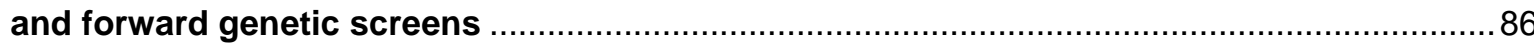

3.3.1 MLG perception does not involve LysM domain containing RLK and RLPs ......................86

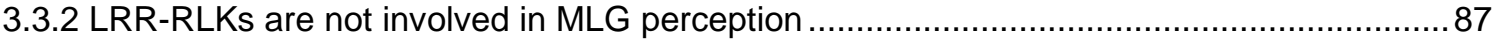

3.3.3 127 tested Arabidopsis ecotypes are MLG-sensitive.................................................... 90

4 Discussion

4.1 Identification and functional characterization of fungal CWDEs ....................................92

4.1.1 Genomic and transcriptomic data reveal $\mathrm{GH}$ family 17 as potentially involved in

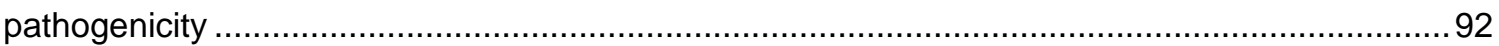

4.1.2 Only BGH06777 could be expressed and purified using the $P$. pastoris expression system .93

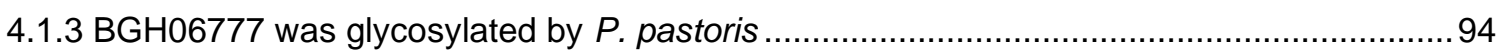

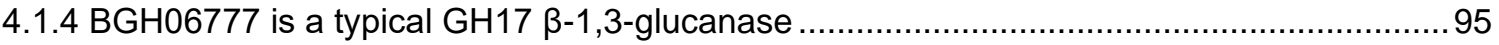

4.1.5 BGH06777 might be involved in papillae degradation ......................................................96

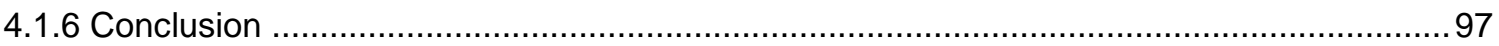

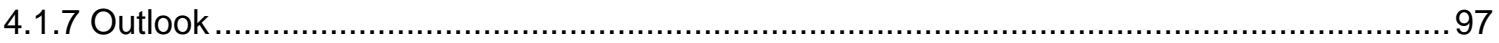

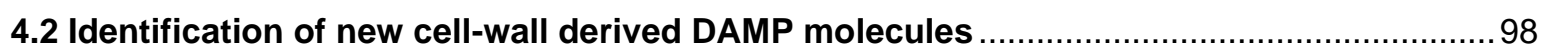

4.2.1 Cellohexaose, xylohexaose and linear $\beta$-1,3-glucan oligosaccharides could slightly induce

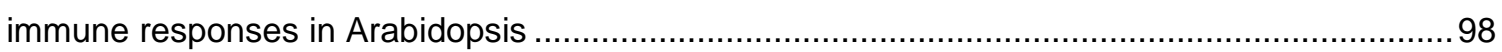

4.2.1.1 Arabidopsis can perceive cellulose-derived oligomers …........................................... 98

4.2.1.2 Xylohexaose elicitation induces the activation of MAPK …........................................99

4.2.1.3 Treatment with linear $\beta$-1,3-glucan oligosaccharides triggers MAPK phosphorylation 100

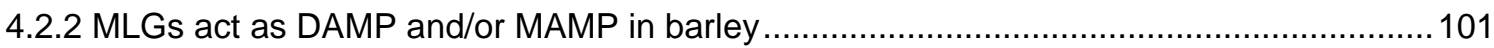

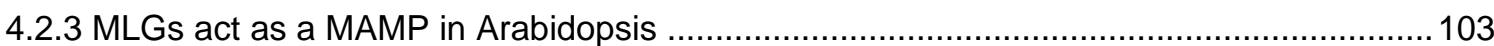

4.2.4 The MLG tetrasaccharide elicits stronger responses than the MLG trisaccharide in Arabidopsis 104

4.2.5 The amplitude and timing of MLG-triggered responses in Arabidopsis differs from chitinand flg22-induced responses 105

4.2.5.1 The calcium peak upon MLG elicitation occurs faster compared to chitin- and

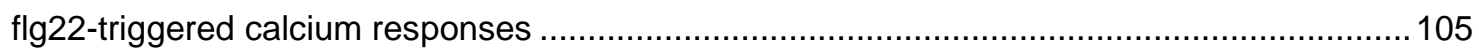

4.2.5.2 MLG perception leads to activation of MAPK6 and MAPK3 ….................................106 
4.2.5.3 Activation of MAPKs and upregulation of WRKY33 and WRKY53 was less induced upon MLG treatment than upon chitin or flg22 elicitation......................................................107

4.2.5.4 MLG oligosaccharides treatment does not influence seedling growth ....................... 108

4.2.6 Reverse genetics analyses reveal that so far unknown molecular components govern MLG

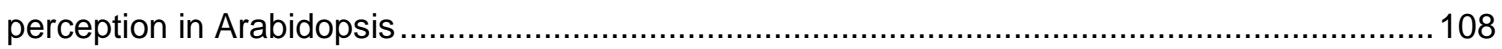

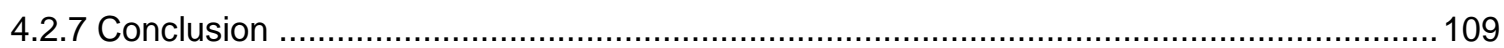

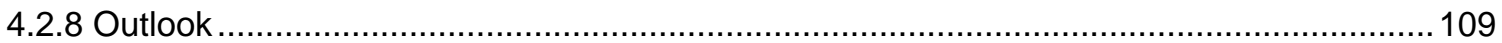

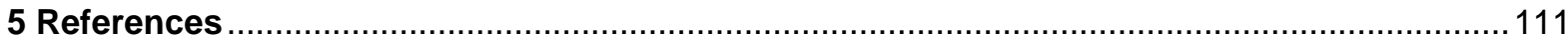

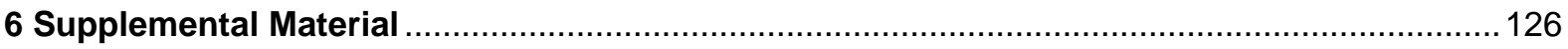

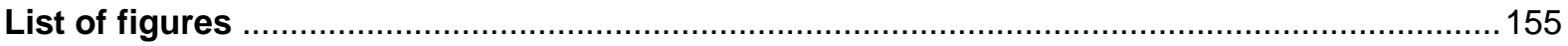

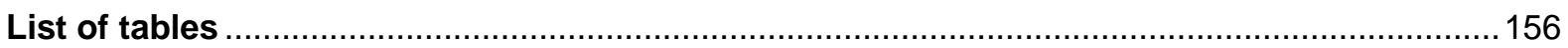

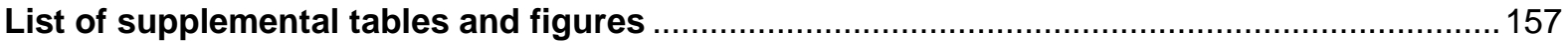

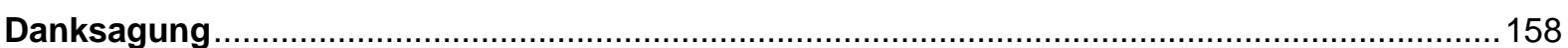

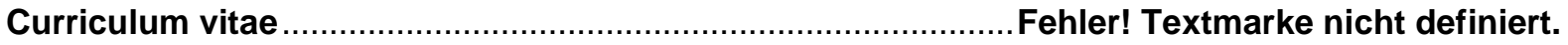




\section{Introduction}

\subsection{The plant immune system}

Plants are constantly exposed to a variety of different microbial pathogens. Microbial pathogens can be bacteria, fungi or oomycetes that exhibit different lifestyles and infection strategies (Jones and Dangl, 2006). In order to protect and defend themselves, plants rely on an innate immune system that includes a variety of defensive barriers and inducible responses (Dodds and Rathjen, 2010).

The first physical barriers that are encountered by microbial pathogens are the cuticle and the plant cell wall (Houston et al., 2016). Pathogens have evolved strategies to overcome the cuticle and the plant cell wall by entering the plant through natural openings such as stomata or wounds, formation of appressoria and/or the secretion of cell wall degrading enzymes (CWDEs) (Chisholm et al., 2006). Once the pathogen has overcome the plant cell wall, it faces the plasma membrane and the two-layered plant immune system. The first layer of plant immunity is referred to as microbe/pathogen associated molecular pattern (MAMP/PAMP)-triggered immunity (MTI/PTI) (Jones and Dangl, 2006; Chisholm et al., 2006). MTI/PTI is based on the recognition of non-self MAMPs, e.g. fungal chitin (Jones and Dangl, 2006). MAMPs are defined as essential and highly conserved molecular structures that cannot easily be modified and are present in a whole class of microbes but absent from host plants (Postel and Kemmerling, 2009). Besides the perception of MAMPs, plants can perceive host-derived molecules, e.g. cell wall derived oligogalacturonides (OGs), that are only abundant upon cell damage or pathogen attack. These host-derived molecules are referred to as damage/danger-associated molecular patterns (DAMPs) (Boller and Felix, 2009). MAMPs and DAMPs are perceived by pattern recognition receptors (PRRs) that reside in the plasma membrane (Figure 1) (Jones and Dangl, 2006; Boller and Felix, 2009). Upon perception of MAMPs or DAMPs, MTI is activated leading to a variety of cellular responses (Boller and Felix, 2009). The defence responses induced upon either MAMP or DAMP recognition are very similar and only differ in their threshold, timing and amplitude (Yu et al., 2017). The induced immune responses can be classified as early and late responses. Early responses are induced within minutes up to half an hour and include the influx of $\mathrm{Ca}^{2+}$ ions into the cytosol, the generation of reactive oxygen species (ROS) by plasma membrane localized NADPH oxidases, phosphorylation of proteins such as mitogen-activated protein kinases (MAPKs) or calcium dependent protein kinases (CDPKs) and transcriptional reprogramming (Boller and Felix, 2009). The activation of late responses occurs within hours and days and includes the inhibition of seedling growth and deposition of callose at the cell wall (Boller and Felix, 2009). PTI is effective against a broad spectrum of pathogens. However, pathogens have evolved strategies to overcome PTI by secreting effector molecules leading to effector triggered susceptibility (ETS) (Figure 1) (Jones and Dangl, 2006). Effectors are molecules that have the ability to change cell structures as well as cell functions and facilitate infection (Selin et al., 2016). They can be active in different cellular compartments and can either act in the apoplast (apoplastic effectors) or in the cytosol (cytoplasmic effectors) (Kamoun, 2006). To secrete effectors, fungal pathogens use haustoria or internal hyphae, while bacterial effectors are delivered directly into the host cell via the type III secretion system (Chatterjee et al., 2013; Selin et al., 2016). Upon secretion, effector molecules can interfere with PTI 
in various ways. Effectors are able to inhibit the kinase activity of certain PRRs (Xiang et al., 2008), dephosphorylate MAPK to inhibit further downstream signaling (Zhang et al., 2007), disturb vesicle trafficking (Block et al., 2008) or prevent the accumulation of the phytohormone salicylic acid, that is involved in defence against biotrophic pathogens (Block et al., 2008). In turn, plants evolved intracellular R-proteins to recognize effectors directly or indirectly by monitoring the status of host targets (Jones and Dangl, 2006) (Figure 1). R-proteins typically contain a nucleotide-binding (NB) domain and a leucine rich repeat (LRR) domain but differ in their $\mathrm{N}$-terminal domain. The $\mathrm{N}$-terminal domain of R-proteins can either contain a Toll, interleukin1-receptor (TIR) or a coiled-coiled (CC) domain (Dodds and Rathjen, 2010). The detection of effectors or the activity of effectors leads to a strong and rapid activation of defence responses which is referred to as effector triggered immunity (ETI) (Figure 1). Defence responses induced upon effector recognition strongly overlap with MAMP/DAMP induced responses but are typically stronger and include a hypersensitive response (HR). HR is programmed cell death and stops the growth of biotrophic pathogens (Jones and Dangl, 2006).

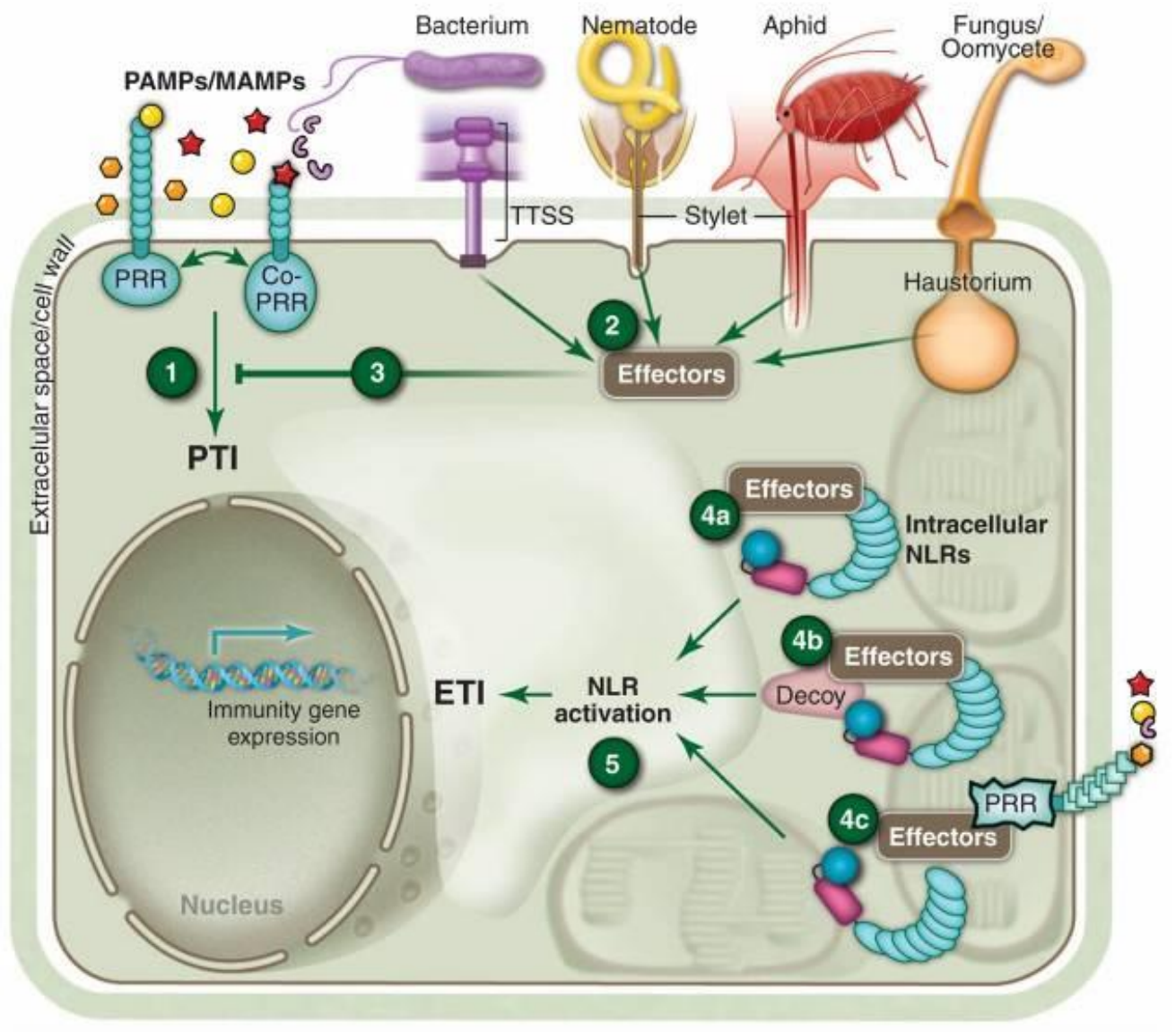

Figure 1. Schematic overview of the two-layered plant immune system. Plasma membrane located pattern recognition receptors (PRRs) perceive pathogen- or microbe associated molecular patterns (PAMPs/MAMPs) which results in the activation of PAMP-triggered immunity (PTI) (1). Pathogens evolved effector molecules that are secreted into the plant cell (2) to suppress PTI (3). The successful suppression of PTI results in effector triggered susceptibility (ETS). Plants, in turn, evolved R-proteins containing a nucleotide-binding domain and a leucine rich repeat domain (NLRs) to detect effectors either directly (4a) or indirectly (4b and 4c). Upon NLR activation (5), effector triggered immunity (ETI) is activated. The figure was adapted from Dangl et al., 2013. 


\subsection{The plant cell wall}

\subsubsection{The structure of the cell wall}

Plant cells are surrounded by a cell wall which is found between the plasma membrane and the middle lamella (Figure 2). The plant cell wall does not only provide shape to the cell but also retains flexibility for cell division. It is involved in intercellular adhesion and communication as the cell wall of young cells is porous and thus allows diffusion of water, hormones and low-molecular weight nutrients (Burton et al., 2010). Furthermore, the plant cell wall forms a structural barrier against various biotic as well as abiotic stresses (Malinovsky et al., 2014).

Typically, two different types of the plant cell wall can be distinguished: the primary and the secondary cell wall. Young growing cells are enclosed by primary cell walls, while secondary cell walls surround cells that have stopped to grow and to divide (Keegstra, 2010; Burton et al., 2010). Both, the primary and the secondary cell wall are composed of a complex matrix of diverse polysaccharides and a comparatively small amount of secreted, cell wall-specific proteins (Lagaert et al., 2009; Zhong and Ye, 2015). The three main classes of polysaccharides found in plant cell walls are cellulose, hemicelluloses and pectic polysaccharides. In brief, cellulose is cross linked to hemicelluloses and embedded in a matrix of pectin (Figure 1) (Lagaert et al., 2009). Notably, although cell walls are composed of these three polysaccharides, the fine structure as well as the three dimensional structure of the plant cell wall differs considerably between different species and different tissues (Burton et al., 2010; Malinovsky et al., 2014).

Cellulose is the most abundant component in primary and secondary cell walls of monocots and dicots and is a homopolymer consisting of $\beta-1,4$-linked glucose monomers. The glucan chains are synthesized individually at the plasma membrane by the cellulose synthase complex that consists of cellulose proteins and other protein complex partners (Keegstra, 2010; McFarlane et al., 2014). Upon synthesis, the glucan chains are able to crystallize into cellulose microfibrils by van der Waals forces or by forming hydrogen bonds (McFarlane et al., 2014). The crystallinity, length and the angle of the cellulose microfibrils in the cell wall mainly determine the physical properties of the cell wall as cellulose is the main polysaccharide (McFarlane et al., 2014).

Hemicelluloses are connected to cellulose microfibrils via hydrogen bonds and are thought to further interconnect and strengthen the cellulose network (Figure 1) (McFarlane et al., 2014). Hemicelluloses represent a diverse group of polysaccharides including xyloglucan, heteroxylans, heteromannans and mixed linkage glucans (MLGs). Disregarding MLGs, hemicelluloses are typically composed of a backbone of $\beta-1,4$-linked hexosyl residues that can be further substituted with various side chains (Pauly et al., 2013). The major hemicellulose present in the primary cell wall of dicots is xyloglucan (Scheller and Ulvskov, 2010). Xyloglucan consists of a backbone of $\beta-1,4$-linked glucose monomers that are decorated with xylosyl residues. In several species, three glycosyl residues are substituted with xylose followed by one unsubstituted glycosyl residue (Park and Cosgrove, 2015). The xylosyl residue can be further substituted with e.g. fucose, galactose, xylose or galacturonic acid (Schultink et al., 2014). Another group of hemicelluloses are heteroxylans. Heteroxylans are the most abundant hemicelluloses in monocot cell walls and secondary cell walls of dicots. Generally, heteroxylans are 


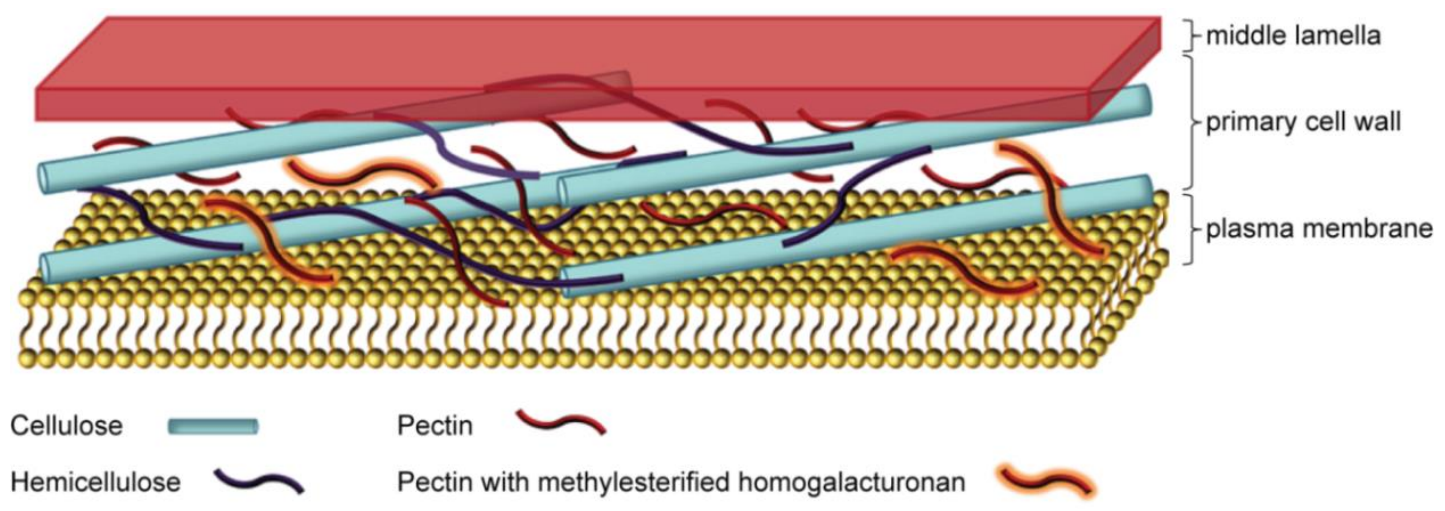

Figure 2. The structure of the primary cell wall. The primary cell wall is composed of cellulose microfibrils, hemicelluloses and pectic polysaccharides. The figure legend and the figure were modified from Malinovsky et al., 2014.

composed of a linear backbone of xylose. The xylose chains can be substituted with various residues e.g. glucuronosyl or arabinose depending on the species and the tissue (Scheller and Ulvskov, 2010). For example, glucuronoxylan has a xylose backbone substituted with glucuronosyl and methyl glucuronosyl residues and is mainly found in dicots, whereas glucuronoarabinoxylan contains arabinofuranosyl as well as methyl-glucuronosyl residues and is mainly found in monocots (Scheller and Ulvskov, 2010; Pauly et al., 2013). The hemicelluloses classified as heteromannans can be further divided into four classes, namely mannan, glucomannan, galactomannan and galactoglucomannan (Pauly et al., 2013). The backbone of mannan and galactomannan is composed of $\beta$-1,4-linked mannose, while glucomannan and galactoglucomannan are composed of glucose and mannose residues. Furthermore, galactosyl residues can be found as side chains in galactomannan as well as galactoglucomannan (Scheller and Ulvskov, 2010). The synthesis of hemicelluloses occurs in the Golgi apparatus and involves several glycosyl transferases (GTs) for the synthesis of mannans and heteroxylans as well as cellulose synthase like family C (CSLC) genes for the synthesis of xyloglucans (Scheller and Ulvskov, 2010; Pauly et al., 2013). MLGs represent an untypical class of hemicelluloses since they are composed of unsubstituted glucose monomers that are connected through both $\beta-1,3$ - and $\beta-1$-4-linkages resulting in a $\beta-1,3 ; 1,4$-polymer. Usually, cellotriosyl or cellotetrasyl units are connected through $\beta$-1,3-linkages (Burton and Fincher, 2014). In higher plants, MLGs are only present in the cell wall of grasses (Pauly et al., 2013). The grass-specific CSL gene families CSLF of rice and CSLH in barley were shown to be involved in MLG synthesis (Burton et al., 2006; Doblin et al., 2009).

Pectic polysaccharides represent the most complex cell wall polysaccharide and can be subdivided into three groups, namely homogalacturonan, rhamnogalacturonan I and rhamnogalacturonan II (Atmodjo et al., 2013). Homogalacturonan and rhamnogalacturonan II are composed of an a-1,4-linked galacturonic acid backbone. In contrast to homogalacturonan which is only partially methylesterified or acetylated, rhamnogalacturonan II can be substituted with four different side chains consisting of 12 different glycosyl residues e.g. methyl xylose (Caffall and Mohnen, 2009; Patova et al., 2014). Rhamnogalacturonan I consists of a backbone of alternating rhamnose and 
galacturonic acid in which galacturonic acid residues can be acetylated. Rhamnogalacturonan I can further be substituted with e.g. arabinan or galactan depending on the cell type and developmental stage (Atmodjo et al., 2013; Patova et al., 2014). Synthesis of pectic polysaccharides takes place in the Golgi and involves several GTs, methyltransferases and acetyltransferases (Atmodjo et al., 2013). Pectic polysaccharides are less prominent in secondary cell walls compared to primary cell walls (Caffall and Mohnen, 2009; Malinovsky et al., 2014). However, secondary cell walls are further reinforced with lignin to enhance the mechanical support (Zhong and Ye, 2015).

\subsubsection{The role of the plant cell wall in plant immunity}

The plant cell wall is not only a passive physical barrier but is also actively modified and reinforced upon pathogen attack (Underwood, 2012). The phenolic polymer lignin is deposited in the cell wall upon pathogen attack and is thought to be involved in cell wall reinforcement (Bellincampi et al., 2014). Also, callose-enriched appositions, called papillae, are formed in close proximity to fungal penetration sites between the cell wall and the plasma membrane (Bacete et al., 2018). Besides the $\beta$-1,3-glucan callose, papillae are composed of pectic polysaccharides, xyloglucan, cell wall structural proteins, peroxidases, ROS and phenolic compounds including lignin (Underwood, 2012). Papillae are thought to reinforce the plant cell wall and thus, slow down invasion of a pathogen. However, the impact of papillae formation and especially callose deposition on plant immunity is not clear (Voigt, 2014; Bacete et al., 2018). Nevertheless, it was shown that the effectiveness of papillae depends on the composition. Papillae that hinder penetration of the obligate powdery mildew Blumeria graminis f.sp. hordei (Bgh) in barley contain a higher amount of cellulose, callose and arabinoxylan compared to papillae that could not stop penetration of the powdery mildew (Chowdhury et al., 2014). Additionally, $\beta-1,3$-glucan oligosaccharides were shown to induce immune responses including the influx of $\mathrm{Ca}^{2+}$ ions and the activation of MAPK in $A$. thaliana (Mélida et al., 2018). In Nicotiana tabacum as well as in Vitis vinifera, the $\beta$-1,3-glucan polymer laminarin was shown to induce e.g. generation of ROS and expression of defence genes (Klarzynski et al., 2000; Aziz et al., 2003). Although elicitor active $\beta$-1,3-glucans might derive from fungal cell walls as it is an abundant fungal cell wall component, it might be possible that callose in papillae represent a source for DAMPs (Mélida et al., 2018).

Besides modifying the plant cell wall, the status of the plant cell wall is monitored. Defence responses are activated upon changes in expression or activity of proteins that play in role in cell wall remodeling and/or synthesis. These alterations can occur upon cell wall damage induced by pathogens (Bacete et al., 2018). Furthermore, the plant cell wall represents a source for DAMPs that may be generated upon action of CWDEs (Bacete et al., 2018). The probably best-studied DAMPs are OGs. OGs are likely derived upon degradation of the pectic polysaccharide homogalacturonan by polygalacturonases (Ferrari et al., 2013). During the early stages of infection, pectin degrading enzymes are secreted and start hydrolyzing pectic polysaccharides. However, plants have polygalacturonase inhibiting proteins (PGIPs) that inhibit the activity of pectin degrading enzymes and 
thereby favor the generation of PTI inducing OGs (De Lorenzo and Ferrari, 2002; Ferrari et al., 2013; Bellincampi et al., 2014). OGs with a length of 10 - 15 were shown to be elicitors of defence responses in plants (Ferrari et al., 2007; Denoux et al., 2008), although shorter fragments with less than 10 residues also exhibit elicitor activity (Davidsson et al., 2017). Upon perception of OGs, the generation of ROS, the phosphorylation of MAPK6 and MAPK3 as well as the transcriptional reprogramming is induced (Galletti et al., 2008; Galletti et al., 2011; Davidsson et al., 2017). Furthermore, A. thaliana plants pre-treated with OGs show an increase in resistance against Botrytis cinerea and Pectobacterium carotovorum (Ferrari et al., 2007; Davidsson et al., 2017). Besides OGs, cellulose-derived oligomers were shown to induce immune responses in $A$. thaliana. Upon treatment of $A$. thaliana with cellobiose several immune responses are activated including influx of $\mathrm{Ca}^{2+}$, activation of MAPK and transcriptional reprogramming. Pretreatment with cellobiose also increased resistance against the bacterial pathogen Pseudomonas syringae pv tomato DC3000 (de Azevedo Souza et al., 2017). Furthermore, the hemicellulose xyloglucan was recently identified as DAMP in $A$. thaliana and $V$. vinifera. Xyloglucan triggers the activation of MAPK and the expression of defence-related genes but not the generation of ROS in $A$. thaliana and V. vinifera (Claverie et al., 2018). In Nicotiana benthaminana and Oryza sativa, mannan oligosaccharides with a length of 2-6 residues trigger the influx of $\mathrm{Ca}^{2+}$ ions, the generation of ROS, the activation of MAPK and lead to an increase in resistance against pathogens. These results indicate that mannan oligosaccharides act as DAMP (Zang et al., 2019). The fact that $A$. thaliana and other plant species can recognize break down products of the plant cell wall and in turn activate immune responses indicate that plants monitor the status of the cell wall and that changes in the cell wall lead to activation of immune responses (de Azevedo Souza et al., 2017; Bacete et al., 2018).

\subsection{Cell wall degrading enzymes}

In order to successfully infect a plant, pathogens have to overcome the plant cell wall (Chisholm et al., 2006; Malinovsky et al., 2014). One of the strategies to overcome the cell wall is the secretion of CWDEs. CWDEs are enzymes that can act on and degrade specific components of the cell wall. Thereby, the cell wall structure is loosened which allows the pathogen to enter (Kubicek et al., 2014). CWDEs can be classified into different enzyme classes based on their function, namely carbohydrate esterases (CEs), polysaccharide lyases (PLs), glycoside hydrolases (GHs) and carbohydrate-binding modules (CBMs) (Carbohydrate Active Enzymes database, http://www.cazy.org/, Lombard et al., 2014). The different enzyme classes can be further divided into families based on sequence similarities which originated from the classification of GHs (Henrissat, 1991). The annotation of enzymes to families according to sequence similarities is based on the concept that the three dimensional structure of a protein depends on the amino acid sequence and that the structure of a protein determines its function (Davies and Henrissat, 1995). Currently, CEs comprise 17 families, while 40 families for PLs and more than 160 families for GHs are described (Carbohydrate Active Enzymes database, http://www.cazy.org/, Lombard et al., 2014). 
CEs are involved in the release of ester-linked acyl groups of substituted carbohydrates, e.g. in pectin methyl esters or acetylated xylan. Furthermore, cutinases that act on cutin are classified as CEs (Nakamura et al., 2017). CEs may use different reaction mechanisms but the most common one is the deacetylation mediated by a serine-histidine-aspartic acid catalytic triad, which is similar to the mechanism employed by lipases (Nakamura et al., 2017). The release of the acylated residues is believed to allow degradation of these polysaccharides since it may facilitate the access of GHs to the polysaccharide (Christov and Prior, 1993).

PLs cleave polysaccharides containing uronic acid, e.g. pectic polysaccharides and hydrolyse $\beta-1,4$-linkages via a $\beta$-elimination mechanism, which is a three stage reaction (Garron and Cygler, 2014). Briefly, as a first step, the carboxyl group of the substrate is neutralized and subsequently, a proton of the fifth carbon atom of the uronic acid is subtracted and an intermediate is formed. As a last step, electrons are removed from the carboxylic group of the substrate which results in the formation of a double bond between the fourth and fifth carbon atom of the substrate and consequently in the cleavage of the glycosidic bond (Michaud et al., 2003).

The hydrolysis of glycosidic linkages in glycosides, e.g. cellulose or xylose, is catalyzed by proteins classified as GH (Vuong and Wilson, 2010). Typically, two reaction mechanisms are found in GHs, which classify them as either retaining $\mathrm{GH}$ or inverting $\mathrm{GH}$. With few exceptions, either retaining or inverting GHs are found in one family (Koshland, 1953; Vuong and Wilson, 2010; Ardèvol and Rovira, 2015). Inverting GHs, use a one-step catalysis in which the nucleophilicity of a water molecule is enhanced by the enzymatic residue acting as base. The water molecule attacks the anomeric center and facilitates cleavage of the glycosidic bond (Figure 3 A) (Vuong and Wilson, 2010). Retaining GHs, however, use a two-step mechanism to catalyze the hydrolysis (Figure $3 \mathrm{~B}$ ). First, a proton is donated from the enzymatic acid residue to the oxygen atom of the substrate and the nucleophile of the enzyme attacks the anomeric center of the substrate. As a consequence, the intermediate is covalently bound to the enzyme and a glycosyl enzyme intermediate is formed. Next, the deprotonated carboxylate behaves as a base and facilitates together with an activated water molecule the hydrolysis of the glycosyl enzyme intermediate resulting in the release of the product (Vuong and Wilson, 2010).

CBMs do not possess catalytic activity themselves, however, are involved in binding of carbohydrates. They can be found at the C- or N-terminus of a CWDE or a carbohydrate active enzyme involved in the synthesis of carbohydrates. CBMs can increase the hydrolytic activity of an enzyme by directing the enzyme to its substrate (Shoseyov et al., 2006). 
A

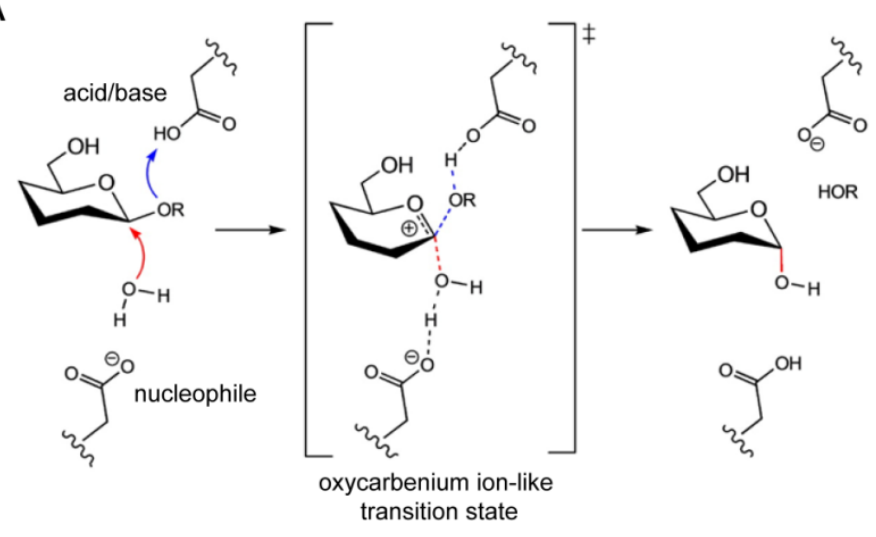

B

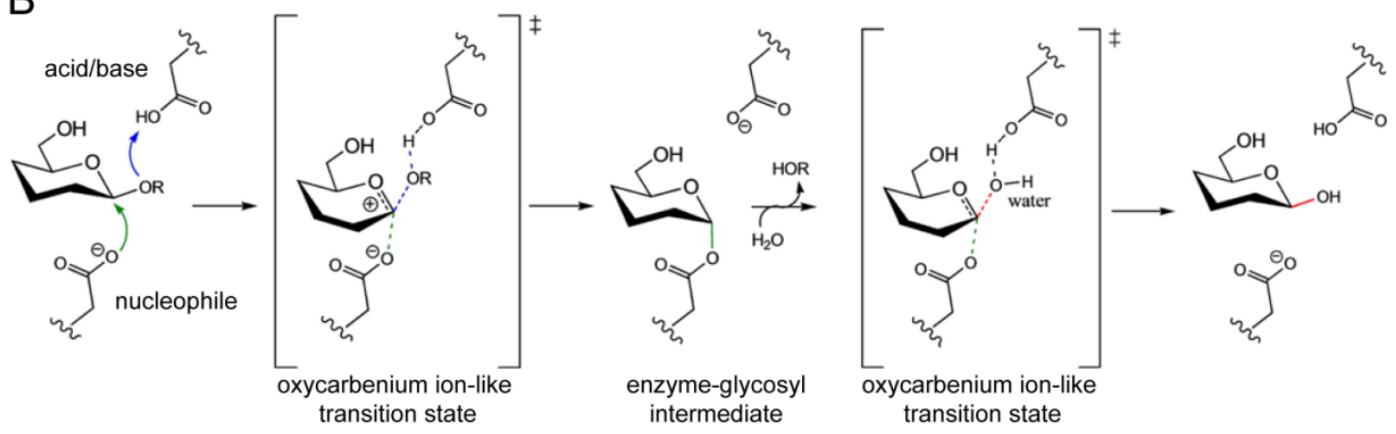

Figure 3. Mechanism of glycoside hydrolysis by GH. (A) Hydrolysis mechanism of inverting GH. (B) Hydrolysis mechanism of retaining GH. Figure and figure legend were modified from Ardèvol and Rovira, 2015.

\subsection{The role of CWDEs in fungal pathogenicity}

Fungal genomes harbour several genes encoding for CWDEs including CEs, GHs that can deconstruct cellulose, hemicelluloses and pectic polysaccharides as well as PLs that act on uronic acid-containing polysaccharide, e.g. on rhamnogalacturonan (Kubicek et al., 2014). However, genome analyses revealed that the arsenal of CWDEs differs between fungal species and that this difference might reflect the lifestyle as well as host specificity of the fungus (King et al., 2011; Zhao et al., 2014). Fungi that infect dicot species are characterized by a higher number of enzymes degrading pectic polysaccharides than fungi infecting monocot plants (Zhao et al., 2014). This observation reflects the fact that cell walls of dicot species contain more pectin than cell walls of monocot species (Kubicek et al., 2014). Furthermore, the number of genes encoding for CWDEs is higher in necrotrophic and hemibiotrophic fungi that eventually kill their host compared to the number of CWDE genes identified in biotrophic fungi, which rely on the living host and typically employ a stealth strategy for infection (Zhao et al., 2014). The obligate biotrophic fungus Bgh is the causal agent of powdery mildew on barley. A genome study conducted in 2010 showed that the genome of Bgh is characterized by a dramatically reduced number of genes encoding for enzymes involved in cell wall degradation. In this study, Bgh was shown to be equipped with only two lignocellulose-degrading enzymes, four hemicellulose degrading enzymes and one pectin $\beta-1,3$-glucan hydrolyzing enzyme but does not possess cellulose-, xylan- or pectin-degrading enzymes (Spanu et al., 2010). 


\subsection{Perception of MAMPs and DAMPs by PRRs}

So far, all known plant PRRs that perceive MAMPs or DAMPs are located at the plasma membrane and are either receptor-like kinases (RLKs) or receptor-like proteins (RLPs) (Macho and Zipfel, 2014; Mélida et al., 2018). RLKs contain an intracellular kinase domain, a single-pass transmembrane domain as well as an ectodomain that is involved in ligand perception. RLPs resemble RLKs structurally, however, they lack an intracellular kinase domain and the attachment to the plasma membrane of RLPs can be facilitated by a transmembrane domain or a GPI-anchor (Monaghan and Zipfel, 2012; Macho and Zipfel, 2014). RLKs and RLPs can be grouped into different categories depending on the domains present in the ectodomain. They can contain leucine-rich repeat domains (LRRs), lysin motifs (LysMs), epidermal growth factor (EGF) domains or lectin motifs. While LRR domain containing RLKs and RLPs were shown to perceive proteinaceous MAMPs, e.g. bacterial flagellin (flg), RLKs or RLPs that contain LysM, EGF-like domains or lectin motifs recognize carbohydrate MAMPs, e.g. fungal chitin (Macho and Zipfel, 2015; Tang et al., 2017). As RLPs lack an intracellular kinase domain, they likely associate with co-receptor RLKs to induce signal transduction. Additionally, the formation of homo- and heterocomplexes of RLK- and RLP-PRRs at the plasma membrane has been shown to be important for ligand perception and signal transduction (Macho and Zipfel, 2014). To transduce the signal from the plasma membrane to downstream signaling components, PRRs and their complex partners need cytoplasmic partners. Receptor-like cytoplasmic kinases (RLCK), that lack an extracellular domain, were shown to be direct targets of PRR complexes and involved in signal transduction (Liang and Zhou, 2018).

\subsubsection{Peptide MAMP perception by LRR-RLKs}

One example for a well-studied LRR-RLK is FLAGELLIN SENSING 2 (FLS2). The LRR-RLK FLS2 perceives the bacterial MAMP flg, the major component of bacterial flagella. FLS2 mediates flg perception in several plant species including Arabidopsis, rice, tomato and tobacco (Gómez-Gómez and Boller, 2000; Robatzek et al., 2007; Hann and Rathjen, 2007; Takai et al., 2008). An N-terminal epitope of flg consisting of 22 amino acids (flg22) is sufficient for perception via FLS2 and the subsequent induction of immune responses (Gómez-Gómez and Boller, 2000; Chinchilla et al., 2006). The influx of $\mathrm{Ca}^{2+}$ ions, the generation of ROS, the activation of MAPK and transcriptional reprogramming represent typical immune responses initiated upon flg22 perception (Monaghan and Zipfel, 2012). Plants that do not have a functional FLS2 were shown to be more susceptible towards bacterial pathogens (Zipfel et al., 2004).

The LRR-RLK ELONGATION FACTOR THERMO UNSTABLE RECEPTOR (EFR) is another well-characterized PRR. EFR perceives the bacterial elongation factor TU (EF-TU), which is highly conserved in bacteria (Zipfel et al., 2006). Epitopes of either 26 or 18 amino acids present at the acetylated N-terminus of EF-TU (elf26 or elf18) are sufficient to trigger MAMP-induced responses (Kunze et al., 2004). The responses induced upon elf18 perception are very similar to responses 
induced by flg22. Furthermore, loss of EFR in $A$. thaliana results in an enhanced susceptibility towards Agrobacterium tumefaciens (Zipfel et al., 2006).

In the last years, it became apparent that PRRs do not act alone but are present in multi-protein complexes at the plasma membrane (Monaghan and Zipfel, 2012; Macho and Zipfel, 2014). The BRI1-ASSOCIATED KINASE1/SOMATIC EMBRYOGENESIS RECEPTOR KINASE 3 (BAK1/SERK3) was initially shown to be involved in brassinosteroid (BR) signaling as a positive regulator of the $B R$ receptor BR INSENSITIVE 1 (BRI1) (Nam and Li, 2002; Li et al., 2002). Furthermore, BAK1 plays a role in immune signaling as an essential component of PRR LRR-RLK complexes (Liebrand et al., 2014). Thus, a defect in BAK1 results in compromised responses towards BR and MAMPs (Li et al., 2002; Chinchilla et al., 2007; Roux et al., 2011). BAK1 is a kinase active LRR-RLK with a short cytoplasmic domain (Liebrand et al., 2014). It directly interacts with the flg22 receptor FLS2 and the elf18 receptor EFR in a ligand dependent manner (Chinchilla et al., 2007; Roux et al., 2011). Upon ligand perception, a heterodimer between BAK1 and either EFR or FLS2 is formed and transphosphorylation events at the intracellular domains occur (Chinchilla et al., 2007; Roux et al., 2011; Heese et al., 2007; Schulze et al., 2010). The transphosphorylation events between BAK1 and the respective PRR LRR-RLK also involve the RLCK BOTRYTIS-INDUCED KINASE 1 (BIK1). BIK1 directly interacts with FLS2, EFR and BAK1. Upon flg22 perception, BIK1 is phosphorylated by BAK1 and subsequently, phosphorylates FLS2 and BAK1. After the transphosphorylation events, BIK1 is released from the complex and activates downstream signaling (Lu et al., 2010; Zhang et al., 2010).

Several LRR-RLPs have been identified as immune receptors in the last years (Jehle et al., 2013a; Jehle et al., 2013b; Zhang et al., 2013; Zhang et al., 2014; Albert et al., 2015). As RLPs lack an intracellular kinase domain, they require a signaling partner to transduce the signal upon ligand perception. The LRR-RLK SUPPRESSOR OF BIR1-1/EVERSHED (SOBIR1/EVR) was shown to interact constitutively with several LRR-RLP in tomato and $A$. thaliana and to be required for their function in plant immunity (Liebrand et al., 2013; Jehle et al., 2013a; Zhang et al., 2013; Zhang et al., 2014). RLPs that form a complex with RLKs are supposed to be bimolecular equivalents to RLKs (Gust and Felix, 2014). In Arabidopsis, the LRR-RLPs RLP1/ReMAx, RLP30, RLP23 as well as RLP42/RBPG1 have been shown to contribute to immunity in a SOBIR1-dependent manner. RLP1/ReMAX perceives the Xanthomonas campestris peptide MAMP eMAX. A sobir mutant lacks the ability to respond to eMAX demonstrating that SOBIR1 is involved in eMAX perception (Jehle et al., 2013a; Jehle, et al., 2013b). NECROSIS- AND ETHYLENE INDUCING PEPTIDE 1 (NEP1)-LIKE PROTEINs (NLPS) are peptidic MAMPs that trigger leaf necrosis. A conserved peptide of 20 amino acids from NLPs (nlp20) is sufficient to induce immune responses and is perceived by RLP23. RLP23 is constitutively found in a complex with SOBIR1 that mediates signal transduction (Bi et al., 2014; Albert et al., 2015). The SCLEROTINIA CULTURE FILTRATE ELICITOR1 (SCFE1) is a proteinaceous elicitor from Sclerotinia sclerotiorum that triggers typical MAMP responses in Arabidopsis and is perceived by RLP30. The activation of typical MAMP responses was shown to be dependent on SOBIR1 as sobir mutants show compromised immune responses upon SCFE1 treatment (Zhang et al., 2013). Furthermore, fungal endopolygalacturonases act as MAMP and are perceived by RLP42/RBPG1. In agreement with the model, RLP42/RBPG1 was shown to 
constitutively interact with SOBIR1 independent from ligand perception and is essential for RBPG1-mediated responses (Zhang et al., 2014). Interestingly, BAK1 is also required for the perception of nlp20, SCFE1 and fungal polygalacturonases (Zhang et al., 2013; Zhang et al., 2014; Albert et al., 2015).

\subsubsection{Perception of carbohydrate MAMPs by LysM domain containing RLKs}

The LysM domain represents a CBM that is important for binding $\mathrm{N}$-acetylglucosamine. In plants, LysM domain containing RLKs have been demonstrated to be involved in symbiosis or plant defence (Antolín-Llovera et al., 2014).

The fungal cell wall component chitin is composed of $\beta$-1,4-linked $N$-acetylglucosamine (Muzzarelli, 1977). Polymeric as well as oligomeric chitin can act as MAMP and induce defence responses in plants (Boller and Felix, 2009).

In O. sativa, chitin perception and subsequent signal transduction is facilitated by CHITIN ELICTOR BINDING PROTEIN (OsCEBiP) and CHITIN ELICITOR RECEPTOR-LIKE KINASE1 (OsCERK1). The plasma membrane residing LysM RLP OsCEBiP is the main chitin receptor and directly binds chitin via its second LysM domain (Kaku et al., 2006; Shimizu et al., 2010; Hayafune et al., 2014). One chitin molecule can bind to two OsCEBiP molecules which leads to the formation of a homodimer. The homodimer might then associate with two molecules of the LySM-RLK OsCERK1 for signal transduction (Hayafune et al., 2014; Shinya et al., 2015). Silencing of OsCEBiP and OsCERK1 leads to a reduction in chitin-induced defence responses and an increase in susceptibility towards fungal pathogens (Kaku et al., 2006; Kishimoto et al., 2010; Shimizu et al., 2010; Kouzai et al., 2014a; Kouzai et al., 2014b). Besides OsCERK1 and OsCEBiP, the OsRLCK185 can be found in the receptor complex. OsRLCK185 is phosphorylated upon chitin perception and triggers subsequent signaling events (Yamaguchi et al., 2013; Wang et al., 2017).

In $A$. thaliana, the LysM-RLK CHITIN ELICITOR RECEPTOR LIKE KINASE1 (CERK1) is crucial for chitin perception and confers resistance to fungal pathogens. Arabidopsis mutants lacking functional CERK1 are dramatically compromised in chitin induced immune responses e.g. the generation of ROS and show enhanced susceptibility towards fungal pathogens (Miya et al., 2007; Wan, et al., 2008b). The ectodomain of CERK1 harbours three LysM domains of which the second LysM domain was shown to directly bind chitin (Miya et al., 2007; Liu et al., 2012b). Binding of polymeric chitin or chitin octamers facilitates homodimerization of CERK1 and consequent phosphorylation of the intracellular domain (Petutschnig et al., 2010; Liu et al., 2012b). As shorter chitooligomers can bind to CERK1 but do not induce CERK1 phosphorylation and consequent immune responses, phosphorylation of CERK1 seems to be indispensable for signal transduction and activation of immune responses (Petutschnig et al., 2010; Liu et al., 2012b). Three OsCEBiP-related proteins are present in Arabidopsis, namely LYM1, LYM2 and LYM3 (LysM-containing receptor-like proteins 1-3) (Antolín-Llovera et al., 2014). LYM1 and LYM3 were demonstrated to be involved in bacterial peptidoglycan signaling (Willmann et al., 2011). The closest homolog of OsCEBiP, LYM2, binds chitin, 
however, is not required for the activation of chitin-triggered immune responses but regulates plasmodesmata flux in response to chitin (Shinya et al., 2012; Faulkner et al., 2013). In a proteomics approach, the LysM-RLKs LYK4 and LYK5 were shown to have chitin binding activity and represent possible receptor complex partners of CERK1 (Petutschnig et al., 2010). Although both, LYK4 and LYK5, have an intracellular kinase domain, they lack kinase activity (Wan et al., 2012; Cao et al., 2014). The knock-out mutant lyk4-1 shows a reduction in chitin induced responses, e.g. induction of chitin responsive genes, demonstrating the involvement of LYK4 in chitin signaling (Wan et al., 2012). The role of LYK5 in chitin signaling, however, is ambiguous. A T-DNA mutant in the Landsberg (Ler) background (lyk5-1) does not show a reduced induction of WRKY53 or MAPK3 upon chitin treatment (Wan et al., 2008b; Wan et al., 2012), but a minor reduction in CERK1 phosphorylation and MAPK activation (Cao et al., 2014). In contrast, a second T-DNA mutant in the Columbia-0 (Col-0) background (lyk5-2) shows reduction of chitin induced responses, e.g. $\mathrm{Ca}^{2+}$ influx (Cao et al., 2014). The single mutants of $l y k 4-1$ and $l y k 5-2$ do not resemble the cerk $1-2$ mutant regarding the reduction of the chitin-triggered immune responses, however, the lyk5-2 lyk4-1 double mutant showed the same reduction in chitin triggered ROS generation and MAPK activation as cerk1-2 (Cao et al., 2014). This demonstrates a role of LYK4 and LYK5 in chitin perception as well as functional redundancy of LYK4 and LYK5 (Cao et al., 2014). Furthermore, LYK5 was shown to form homodimers in the plasma membrane in a ligand-independent manner and to associate with CERK1 upon chitin perception (Cao et al., 2014). Based on these results, Cao et al (2014) proposed a new model for chitin perception: LYK5 is present at the plasma membrane as a homodimer without a stimulus. Upon chitin perception, LYK5 binds to CERK1 to form a heterotetramer consisting of two LYK5 and two CERK1 molecules. Consequently, CERK1 will be phosphorylated which is required for signal transduction (Cao et al., 2014).

Another carbohydrate MAMP is peptidoglycan. It is an abundant cell wall component of Gram-positive and Gram-negative bacteria and is composed of alternating $\beta$-1,4-linked $N$-acetylglucosamine and $\mathrm{N}$-acetylmuramic acid moieties (Lovering et al., 2012). In tobacco, rice and Arabidopsis peptidoglycan act as a MAMP, however, receptors for peptidoglycan have only been identified in rice and Arabidopsis (Gust, 2015). In rice, the two LysM-RLPs OsLYP4 and OsLYP6, which are homologs of OsCEBiP, as well as OsCERK1 are required for peptidoglycan perception (Liu et al., 2012a; Ao et al., 2014). Silencing of either OsLYP4 or OSLYP6 results in a reduction of peptidoglycan induced responses and higher susceptibility towards bacterial pathogens (Liu et al., 2012a). In the absence of a stimulus, OSLYP4 and OSLYP6 form a complex at the plasma membrane. Upon peptidoglycan perception, OsLYP4 and OsLYP6 dissociate and recruit OsCERK1 for signal transduction (Ao et al., 2014). In Arabidopsis, LYM1 and LYM3 as well as CERK1 were shown to be critical components of peptidoglycan perception. LYM1 and LYM3 reside in the plasma membrane and might build a peptidoglycan binding module. Upon peptidoglycan binding, CERK1 is recruited and might be required to transduce the signal from the extracellular to the intracellular space (Willmann et al., 2011). 
Since CERK1 is involved in the perception of several carbohydrate MAMPs, it was proposed that CERK1 acts as a co-receptor in plasma membrane complexes analogously to BAK1 (GimenezIbanez et al., 2009; Postel and Kemmerling, 2009).

\subsubsection{Perception of DAMPs}

Besides MAMPs, plants can perceive host derived DAMP molecules which lead to the activation of immune responses. In 2006, a family of plant peptides in Arabidopsis was identified that induce the activation of immune responses, namely Peps (plant elicitor peptides) (Huffaker et al., 2006). Active Peps might be derived from the precursor peptides PROPEPs which are small proteins of about 100 amino acids. However, whether PROPEPs need to be cleaved for activation is still under debate (Bartels and Boller, 2015). In Arabidopsis, the family of PROPEPs comprises eight members. All eight Peps were shown to be able to induce immune responses which were dependent on the plasma membrane residing LRR-RLKs PEP receptor 1 (PEPR1) and PEPR2. Consequently, a double mutant lacking PEPR1 and PEPR2 was shown to be insensitive to all Peps (Huffaker et al., 2006; Yamaguchi et al., 2006; Bartels et al., 2013). Similar to FLS2 and EFR, PEPRs associate with the co-receptor BAK1 upon binding of the elicitor to the LRR domain and both, BAK1 and PEPRs, are subsequently phosphorylated similar to FLS2 and EFR (Tang et al., 2015). Furthermore, the RLCK BIK1 is present in the receptor complex and gets phosphorylated upon ligand binding. Subsequently, BIK1 might dissociate from the complex and mediate downstream signaling (Liu et al., 2013). PROPEPs have also been identified in several angiosperms including crop plants (Bartels and Boller, 2015).

Short fragments composed of $\alpha-1,4$-linked galacturonic acid, called OGs, represent probably the best characterized plant DAMP and were already shown to induce immune responses in the 1980s (Ferrari et al., 2013). OGs might be released upon degradation of the cell wall component homogalacturonan by microbial polygalacturonases (Ferrari et al., 2013). The RLK wall-associated kinase 1 (WAK1) was shown to bind OGs via its N-terminal pectin binding domain (Decreux and Messiaen, 2005). The binding of OGs to WAK1 was stronger when dimers in a calcium-mediated egg-box confirmation were present (Cabrera et al., 2008). Furthermore, a domain swap approach suggests that WAK1 is involved in the perception of OGs. In a chimera consisting of the WAK1 ectodomain and the EFR kinase domain, the kinase domain was stimulated upon OG elicitation. Furthermore, the WAK1 kinase domain was activated upon elf18 treatment in a chimera consisting of the WAK1 kinase domain and the EFR ectodomain (Brutus et al., 2010).

Recently, $\beta-1,3-$ glucan oligosaccharides were shown to induce immune responses in Arabidopsis (Mélida et al., 2018). $\beta$-1,3-glucans are present in the plant in form of callose but are also abundant in the fungal cell wall, therefore might act as MAMP or DAMP. The mutant cerk1-2, lacking the CERK1 receptor, did not show activation of immune responses upon $\beta$-1,3-glucan elicitation indicating that CERK1 is involved in $\beta$-1,3-glucan oligosaccharides perception (Mélida et al., 2018). 


\subsection{Thesis Aims}

The plant cell wall is built of a complex network of cellulose, various hemicelluloses, pectic polysaccharides and glycoproteins and represents a physical barrier to invasive pathogens. In order to infect a plant, pathogens need to overcome the plant cell wall. To accomplish this, pathogens use penetration structures (appressoria) and/or secrete CWDEs (Chisholm et al., 2006). However, information about the role of CWDEs in the pathogenicity of biotrophic fungi is still missing. Therefore, the first aim of this work was to identify and functionally characterize CWDEs of the obligate biotrophic barley powdery mildew $B g h$ that may be required for pathogenicity. To this end, genomic as well as public available transcriptomic data should be analysed regarding potentially secreted and highly expressed GH families. Selected candidate genes should be expressed heterologously in Pichia pastoris and the respective substrates should be identified. Furthermore, the $\mathrm{pH}$ optimum and temperature optimum, Michaelis-Menten kinetics as well as the hydrolysis mode of the recombinant proteins should be determined.

The action of CWDEs during the infection process is believed to result in the generation of cell-wall derived oligosaccharides (Bacete et al., 2018). It is conceivable to postulate that plants evolved the capacity to perceive these cell-wall derived oligosaccharides resulting in the activation of immune responses. Thus, the second aim of this project was to identify new cell-wall derived DAMPs. Therefore, a collection of cell-wall derived oligo- and polysaccharides was purchased and analysed with regard to their ability to induce a $\mathrm{Ca}^{2+}$ influx in the Arabidopsis ecotype Col-0. As Arabidopsis ecotypes differ in their receptor repertoire, the generation of ROS as well as the activation of MAPK should be analysed upon oligo- or polysaccharide treatment in the three ecotypes Col-0, Wassilewskija-0 (Ws-0) and Ws-4. Furthermore, the DAMP activity of monocot specific poly- and oligosaccharides was analysed in barley by testing the generation of ROS and the activation of MAPK. Upon validation of candidate DAMPs, forward and reverse-genetic approaches should be used to identify molecular components involved in the perception of newly identified DAMP molecules. 


\section{Material and Methods}

\subsection{Material}

\subsubsection{Plants}

\subsubsection{Arabidopsis thaliana}

T-DNA mutants and transgenic lines that were used in this work are listed in Table 1 and Table 2, respectively. Arabidopsis thaliana accessions that were used in this study are listed in Table 3.

Table 1. List of T-DNA insertion and transgenic lines used in this work.

\begin{tabular}{ccccc}
\hline Allele & AGI locus & Accession & T-DNA & Reference/Source \\
\hline cerk1-2 & At3g21630 & Col-0 & GABI_096F09 & Miya et al., 2007 \\
\hline fls2c & AT5G46330 & Col-0 & SAIL_691_C4 & Cyril Zipfel \\
\hline efr1 & At5g20480 & Col-0 & SALK_044334 & Zipfel et al., 2006 \\
\hline bak1-4 & AT4G33430.2 & Col-0 & SALK_116202 & $\begin{array}{c}\text { Chinchilla } \text { et al., } \\
\text { 2007 }\end{array}$ \\
\hline bak1-5 & AT4G33430.2 & Col-0 & - & $\begin{array}{c}\text { Schwessinger } \text { et al., } \\
\text { 2011 }\end{array}$ \\
\hline sobir1-12 & AT2G31880.1 & Col-0 & SALK_050715 & - \\
\hline sobir1-14 & AT2G31880.1 & Col-0 & GABI-Kat_643F07 & - \\
\hline lyk5-2 lyk4-2 & At2g33580 & Col-0 & SALK_131911C & PhD Thesis, Jan \\
Et2g23770 & AT3G01840 & Col-0 & SALK_152226 & - \\
\hline lyk2-1 & At2g12170 & Col-0 & SAIL 343B03 & Shinya et al., 2012 \\
\hline lym2-1 & At2g12170 & Col-0 & GABI-Kat 165 H02 & - \\
\hline lym2-4 & At5g62150 & Col-0 & SALK_144729 & - \\
\hline lyt1-1 & & & &
\end{tabular}

Table 2. List of transgenic Arabidopsis lines used in this work.

\begin{tabular}{ccc}
\hline Background & Construct & Reference/Source \\
\hline Col-0 & 35S::mcherry-AEQ & R. Panstruga ${ }^{2}$ \\
\hline
\end{tabular}

\footnotetext{
${ }^{2}$ Ralph Panstruga, Institute for Biology I, Unit of Molecular Cell Biology, RWTH Aachen
} 
Table 3. List of Arabidopsis thaliana accessions used in this work.

\begin{tabular}{|c|c|c|c|}
\hline Accession & Abbreviation & Source & GWAS-ID \\
\hline Aua/Rhon & Aa-0 & Till Ischebeck ${ }^{3}$ & 700 \\
\hline Argentat & $\mathrm{Ag}-0$ & N901 & - \\
\hline Achkarren & Ak-1 & Till Ischebeck ${ }^{3}$ & 6987 \\
\hline Ameland-Firehouse & Amel-1 & Till Ischebeck ${ }^{3}$ & 6990 \\
\hline Antwerpen & $A n-1$ & Till Ischebeck ${ }^{3}$ & 6898 \\
\hline Angleur & Ang-0 & Till Ischebeck ${ }^{3}$ & 6992 \\
\hline Annecy & Ann-1 & Till Ischebeck ${ }^{3}$ & 6994 \\
\hline Blackmount & $\mathrm{Ba}-1$ & Till Ischebeck ${ }^{3}$ & 7014 \\
\hline Baarlo & Baa-1 & Till Ischebeck ${ }^{3}$ & 7002 \\
\hline Blanes & Bla-1 & Till Ischebeck ${ }^{3}$ & 7015 \\
\hline Boot & Boot-1 & Till Ischebeck ${ }^{3}$ & 7026 \\
\hline Borky-1 & Bor-1 & Till Ischebeck ${ }^{3}$ & 5837 \\
\hline Borky-4 & Bor-4 & Till Ischebeck ${ }^{3}$ & 6903 \\
\hline Brunn & $\mathrm{Br}-0$ & Till Ischebeck ${ }^{3}$ & 6904 \\
\hline Basel & Bs-1 & Till Ischebeck ${ }^{3}$ & 8270 \\
\hline Buchschlag & Bsch-0 & Till Ischebeck ${ }^{3}$ & 7031 \\
\hline Burghaun & $\mathrm{Bu}-0$ & Till Ischebeck ${ }^{3}$ & 8271 \\
\hline Burren & Bur-0 & N1028 & - \\
\hline Canary Island & Can-0 & N1064 & - \\
\hline Chateaudun & Chat-1 & Till Ischebeck ${ }^{3}$ & 7071 \\
\hline Ascot-17 & CIBC-17 & Till Ischebeck ${ }^{3}$ & 6907 \\
\hline Ascot-5 & CIBC-5 & Till Ischebeck ${ }^{3}$ & 6730 \\
\hline- & Co & Till Ischebeck ${ }^{3}$ & 7081 \\
\hline Columbia-0 & Col-0 & $\begin{array}{l}\text { J. Dangl, University of } \\
\text { North Carolina, USA. }\end{array}$ & - \\
\hline Compiegne & Com-1 & Till Ischebeck ${ }^{3}$ & 7092 \\
\hline Catania & Ct-1 & N1094 & - \\
\hline Cape Verdi Islands & Cvi-0 & Till Ischebeck ${ }^{3}$ & 6911 \\
\hline
\end{tabular}

3 Till Ischebeck, Department of Plant Biochemistry, Albrecht-von-Haller institute for plant sciences, Göttingen, Germany 


\begin{tabular}{|c|c|c|c|}
\hline Darmstadt & Da-0 & Till Ischebeck ${ }^{3}$ & 7094 \\
\hline Dem & Dem-4 & Till Ischebeck ${ }^{3}$ & 8233 \\
\hline Drall & Drall-1 & Till Ischebeck ${ }^{3}$ & 8284 \\
\hline Dralll & Dralll-1 & Till Ischebeck ${ }^{3}$ & 8285 \\
\hline Duk & Duk & Till Ischebeck ${ }^{3}$ & 6008 \\
\hline Edinburgh & Edi-0 & N1122 & - \\
\hline Eifel & $\mathrm{Ei}-2$ & Till Ischebeck ${ }^{3}$ & 6915 \\
\hline East Malling & Ema-1 & Till Ischebeck ${ }^{3}$ & 5736 \\
\hline St. Maria d. Feiria & Fei-0 & Till Ischebeck ${ }^{3}$ & 8215 \\
\hline Gudow & $\mathrm{Gd}-1$ & Till Ischebeck ${ }^{3}$ & 8296 \\
\hline Geleen & Gel-1 & Till Ischebeck ${ }^{3}$ & 7143 \\
\hline Gieben & Gie-0 & Till Ischebeck ${ }^{3}$ & 7147 \\
\hline Goettingen & Got-22 & Till Ischebeck ${ }^{3}$ & 6920 \\
\hline Glueckingen & Gu-0 & Till Ischebeck ${ }^{3}$ & 6922 \\
\hline La Miniere & Gy-0 & Till Ischebeck ${ }^{3}$ & 8214 \\
\hline Heythuysen & Hey-1 & Till Ischebeck ${ }^{3}$ & 7166 \\
\hline Chisdra & $\mathrm{Hi}-0$ & Till Ischebeck ${ }^{3}$ & 8304 \\
\hline Hannover/Stroehen & $\mathrm{Hs}-\mathrm{O}$ & Till Ischebeck ${ }^{3}$ & 8310 \\
\hline Horni Smrcne & $\mathrm{HSm}$ & Till Ischebeck ${ }^{3}$ & 8236 \\
\hline Innsbruck & $\ln -0$ & Till Ischebeck ${ }^{3}$ & 8311 \\
\hline Isenburg & Is-0 & Till Ischebeck ${ }^{3}$ & 8312 \\
\hline Jena & Je-0 & Till Ischebeck ${ }^{3}$ & 7181 \\
\hline Vranov u Brna & $\mathrm{Jl}-3$ & Till Ischebeck ${ }^{3}$ & 7424 \\
\hline Jamolice & $\mathrm{Jm}-0$ & Till Ischebeck ${ }^{3}$ & 8313 \\
\hline Kelsterbach & Kelsterbach4 & Till Ischebeck ${ }^{3}$ & 8420 \\
\hline Killean & Kil-0 & Till Ischebeck ${ }^{3}$ & 7192 \\
\hline Kindalville & Kin-0 & Till Ischebeck ${ }^{3}$ & 6926 \\
\hline Köln & $\mathrm{Kl}-5$ & Till Ischebeck ${ }^{3}$ & 7199 \\
\hline Kaunas & $\mathrm{Kn}-0$ & N1286 & - \\
\hline Khurmatov & Kondara & Till Ischebeck ${ }^{3}$ & 6929 \\
\hline Karagandy & $K z-9$ & Till Ischebeck ${ }^{3}$ & 6931 \\
\hline
\end{tabular}




\begin{tabular}{|c|c|c|c|}
\hline Loch Ness & LC-0 & Till Ischebeck ${ }^{3}$ & 8323 \\
\hline Landsberg & Ler-1 & Till Ischebeck ${ }^{3}$ & 6932 \\
\hline Limburg & Li-7 & Till Ischebeck ${ }^{3}$ & 7231 \\
\hline Lipowiec & Lip-0 & N1136 & - \\
\hline Lisse & Lisse & Till Ischebeck ${ }^{3}$ & 8430 \\
\hline Llagostera & LL-0 & Till Ischebeck ${ }^{3}$ & 6933 \\
\hline Le Mans & Lm-2 & Till Ischebeck ${ }^{3}$ & 8329 \\
\hline Lipovec & Lp2-2 & Till Ischebeck ${ }^{3}$ & 7520 \\
\hline Lund & Lu-1 & Till Ischebeck ${ }^{3}$ & 8334 \\
\hline Mühlen & Mh-0 & N1368 & - \\
\hline Miramare & Mir-0 & Till Ischebeck ${ }^{3}$ & 8337 \\
\hline Mainz & Mnz-0 & Till Ischebeck ${ }^{3}$ & 7244 \\
\hline Martuba & Mt-0 & N1380 & - \\
\hline Merzhausen & $\mathrm{Mz}-0$ & Till Ischebeck ${ }^{3}$ & 6940 \\
\hline Ascot-10 & NFA-10 & Till Ischebeck ${ }^{3}$ & 6943 \\
\hline Ascot-8 & NFA-8 & Till Ischebeck ${ }^{3}$ & 6944 \\
\hline Nossen & No-0 & N77128 & - \\
\hline Nieps & $\mathrm{Np}-0$ & Till Ischebeck ${ }^{3}$ & 7268 \\
\hline Neuweilnau & $\mathrm{Nw}-0$ & Till Ischebeck ${ }^{3}$ & 8348 \\
\hline Oberursel & Ob-0 & N1418 & - \\
\hline Oldenburg & Old-1 & Till Ischebeck ${ }^{3}$ & 7280 \\
\hline Bou Roubianne & Or-0 & Till Ischebeck ${ }^{3}$ & 7282 \\
\hline Ovelgoenne & Ove-0 & Till Ischebeck ${ }^{3}$ & 7287 \\
\hline Oystese & Oy-0 & N1436 & - \\
\hline Perm & Per-1 & Till Ischebeck ${ }^{3}$ & 8354 \\
\hline Corscalla & PHW-2 & Till Ischebeck ${ }^{3}$ & 8243 \\
\hline Pitztal & $\mathrm{Pi}-0$ & N1454 & - \\
\hline Playa de Aro & Pla-0 & Till Ischebeck ${ }^{3}$ & 7300 \\
\hline Poppelsdorf & Po-0 & N1470 & - \\
\hline Point Grey & Pog-0 & Till Ischebeck ${ }^{3}$ & 7306 \\
\hline Prudka2-23 & Pu2-23 & Till Ischebeck ${ }^{3}$ & 6951 \\
\hline
\end{tabular}




\begin{tabular}{|c|c|c|c|}
\hline Prudka2-7 & Pu2-7 & Till Ischebeck ${ }^{3}$ & 6956 \\
\hline Randan & Ra-0 & Till Ischebeck ${ }^{3}$ & 6958 \\
\hline Rodenbach & $\mathrm{Rd}-0$ & Till Ischebeck ${ }^{3}$ & 8366 \\
\hline St. Josephs & $R m x-180$ & Till Ischebeck ${ }^{3}$ & 7525 \\
\hline Rouen & Rou-0 & Till Ischebeck ${ }^{3}$ & 7320 \\
\hline Rschew & Rsch-4 & N1494 & - \\
\hline San Eleno & Se-0 & Till Ischebeck ${ }^{3}$ & 6961 \\
\hline Seatlle & Seatlle-0 & Till Ischebeck ${ }^{3}$ & 8245 \\
\hline San Feliu & Sf-2 & N1516 & - \\
\hline St. Georgen & $\mathrm{Sg}-1$ & Till Ischebeck ${ }^{3}$ & 7344 \\
\hline Pamiro-Alay & Sha & Till Ischebeck ${ }^{3}$ & 6962 \\
\hline Siegen & $\mathrm{Si}-0$ & Till Ischebeck ${ }^{3}$ & 7337 \\
\hline Sorbo & Sorbo & N931 & - \\
\hline Ascot-1 & $\mathrm{Sq}-1$ & Till Ischebeck ${ }^{3}$ & 6966 \\
\hline Ascot-8 & $\mathrm{Sq}-8$ & Till Ischebeck ${ }^{3}$ & 6967 \\
\hline Stockholm & St-0 & Till Ischebeck ${ }^{3}$ & 8387 \\
\hline Tabor & Ta-0 & - & - \\
\hline Tammisari & Tamm-27 & Till Ischebeck ${ }^{3}$ & 6969 \\
\hline Tossa de Mar & Ts-1 & Till Ischebeck ${ }^{3}$ & 6970 \\
\hline Tsu & Tsu-0 & N1564 & - \\
\hline Umkirch & Uk-1 & Till Ischebeck ${ }^{3}$ & 7378 \\
\hline Ullstorp & UII2-3 & Till Ischebeck ${ }^{3}$ & 6973 \\
\hline Ottenhof & Uod-1 & Till Ischebeck ${ }^{3}$ & 6975 \\
\hline Utrecht & Utrecht & Till Ischebeck ${ }^{3}$ & 7382 \\
\hline $\begin{array}{l}\text { University of British } \\
\text { Columbia }\end{array}$ & Van-0 & Till Ischebeck ${ }^{3}$ & 6977 \\
\hline Warschau & Wa-1 & Till Ischebeck ${ }^{3}$ & 6978 \\
\hline $\begin{array}{c}\text { Lincoln Woods State } \\
\text { Park }\end{array}$ & WAR & Till Ischebeck ${ }^{3}$ & 7477 \\
\hline Westercelle & Wc-1 & Till Ischebeck ${ }^{3}$ & 7404 \\
\hline Weningen & Wei-0 & Till Ischebeck ${ }^{3}$ & 6979 \\
\hline Wilna-1 & Wil-1 & Till Ischebeck ${ }^{3}$ & 0 \\
\hline Wilna-2 & Wil-2 & N1596 & - \\
\hline
\end{tabular}




\begin{tabular}{cccc}
\hline Wassilewskija-0 & Ws-0 & & - \\
\hline Wassilewskija-4 & Ws-4 & & - \\
\hline Wietze & Wt-5 & N1612 & - \\
\hline Wurzburg & Wu-0 & Till Ischebeck ${ }^{3}$ & 6984 \\
\hline Zdarec & Zdr-1 & N1626 & - \\
\hline Zurich & Zu-0 & & \\
\hline
\end{tabular}

\subsubsection{Hordeum vulgare}

For experiments with $H$. vulgare, the cultivar $H$. vulgare cv. Golden Promise was used.

\subsubsection{Bacterial and Yeast Strains}

\subsubsection{Escherichia coli}

For cloning purposes chemically competent E. coli TOP10 F' cells (Invitrogen, Carlsbad, USA) [proAB, laclq, lacZ $\Delta \mathrm{M} 15, \mathrm{Tn} 10$ (TetR)\} mcrA, $\Delta$ (mrrhsdRMS-mcrBC), $\varphi 80$ lacZ $\Delta \mathrm{M} 15, \Delta / a c X 74$, deoR, recA1, $\lambda$-araD139, $\Delta($ ara-leu)7697, gaN , galK, rpsL(StrR), endA1, nupG] were used.

\subsubsection{Pichia pastoris}

For protein expression the Pichia pastoris wild-type strain X-33 (Invitrogen, Carlsbad, USA) [Mut+] was used.

\subsubsection{Vectors}

A list of vectors used or generated in this work is depicted in Table 4.

Table 4. List of vectors used/generated in this work.

\begin{tabular}{|c|c|c|c|}
\hline Name & Description & $\begin{array}{l}\text { Selection } \\
\text { marker }\end{array}$ & Reference/Source \\
\hline $\mathrm{pPICZ} \alpha A$ & $\begin{array}{l}\text { Vector for expression of proteins in } \\
\text { Pichia pastoris }\end{array}$ & Zeocin $^{T M}$ & $\begin{array}{c}\text { Invitrogen } \\
\text { (Carlsbad, USA) }\end{array}$ \\
\hline pPICZaA-Bgh00219 & $\begin{array}{l}\text { Vector for expression of Bgh00219 } \\
\text { cDNA with a C-terminal myc epitope } \\
\text { and polyhistidine-tag under control of } \\
\text { the AOX1 promoter }\end{array}$ & Zeocin $^{T M}$ & This work \\
\hline pPICZaA-Bgh00220 & $\begin{array}{l}\text { Vector for expression of Bgh00220 } \\
\text { cDNA with a C-terminal myc epitope } \\
\text { and polyhistidine-tag under control of } \\
\text { the AOX1 promoter }\end{array}$ & Zeocin $^{T M}$ & This work \\
\hline pPICZaA-Bgh00734 & $\begin{array}{l}\text { Vector for expression of Bgh00734 } \\
\text { cDNA with a C-terminal myc epitope } \\
\text { and polyhistidine-tag under control of } \\
\text { the } A O X 1 \text { promoter }\end{array}$ & Zeocin $^{T M}$ & This work \\
\hline
\end{tabular}




\begin{tabular}{cccc}
\hline pPICZaA-Bgh00736 & $\begin{array}{c}\text { Vector for expression of Bgh00736 } \\
\text { cDNA with a C-terminal myc epitope } \\
\text { and polyhistidine-tag under control of } \\
\text { the AOX1 promoter }\end{array}$ & Zeocin ${ }^{\text {TM }}$ & This work \\
\hline pPICZaA-Bgh06777 & $\begin{array}{c}\text { Vector for expression of Bgh06777 } \\
\text { cDNA with a C-terminal myc epitope } \\
\text { and polyhistidine-tag under control of } \\
\text { the AOX1 promoter }\end{array}$ & Zeocin $^{\text {TM }}$ & This work \\
\hline
\end{tabular}

\subsubsection{Oligonucleotides}

The oligonucleotides in this study were ordered from either Invitrogen (Darnstadt, Germany) or Integrated DNA Technologies (lowa, USA). The lypohilized primers were diluted with ultrapure water to a stock concentration of $100 \mu \mathrm{M}$. Aliquots with a concentration of $10 \mu \mathrm{M}$ were prepared with ultrapure water for standard usage. Oligonucleotides were stored at $-20^{\circ} \mathrm{C}$. All oligonucleotides used in this study are listed in Table 5.

Table 5. List of oligonucleotides used in this work.

\begin{tabular}{|c|c|c|}
\hline Primer & Sequence & Purpose \\
\hline \multicolumn{3}{|c|}{ Oligonucleotides used for cloning } \\
\hline $\begin{array}{l}\text { pPICZaA- } \\
\text { forward }\end{array}$ & AGCTTCAGCCTCTCTTTTCTC & $\begin{array}{l}\text { Amplification of pPICZaA for Gibson } \\
\text { Assembly }\end{array}$ \\
\hline $\begin{array}{l}\text { pPICZ } \alpha A \text { - } \\
\text { reverse }\end{array}$ & $\begin{array}{l}\text { GAACAAAAACTCATCTCAGAAGAGGAT } \\
\text { C }\end{array}$ & $\begin{array}{l}\text { Amplification of pPICZaA for Gibson } \\
\text { Assembly }\end{array}$ \\
\hline $\begin{array}{l}\text { Bgh00220- } \\
\text { Gibson- } \\
\text { forward }\end{array}$ & $\begin{array}{l}\text { AGA AAA GAG AGG CTG AAG CTC GTT } \\
\text { TGA ATG GCT TCA ATG }\end{array}$ & $\begin{array}{l}\text { Amplification of Bgh00220 for cloning into } \\
\text { pPICZaA via Gibson Assembly }\end{array}$ \\
\hline $\begin{array}{l}\text { Bgh00220- } \\
\text { Gibson- } \\
\text { reverse }\end{array}$ & $\begin{array}{l}\text { TCT GAG ATG AGT TTT TGT TCG CAA } \\
\text { GAA AGG TAG TAC AG }\end{array}$ & $\begin{array}{l}\text { Amplification of Bgh00220 for cloning into } \\
\text { pPICZaA via Gibson Assembly }\end{array}$ \\
\hline $\begin{array}{l}\text { Bgh06777- } \\
\text { Gibson- } \\
\text { forward }\end{array}$ & $\begin{array}{l}\text { AGAAAAGAGAGGCTGAAGCTTATTGGA } \\
\text { AAGGATTTAACGC }\end{array}$ & $\begin{array}{l}\text { Amplification of Bgh06777 for cloning into } \\
\text { pPICZaA via Gibson Assembly }\end{array}$ \\
\hline $\begin{array}{l}\text { Bgh06777- } \\
\text { Gibson- } \\
\text { reverse }\end{array}$ & $\begin{array}{l}\text { TCTGAGATGAGTTTTTGTTCACAGGAGA } \\
\text { GATCGTATTG }\end{array}$ & $\begin{array}{l}\text { Amplification of Bgh06777 for cloning into } \\
\text { pPICZaA via Gibson Assembly }\end{array}$ \\
\hline \multicolumn{3}{|c|}{ Oligonucleotides for Expression Analysis } \\
\hline ActinF & TGCGACAATGGAACTGGAATG & Semi-quantitative RT-PCR of $A C T I N 1$ \\
\hline ActinR & GGATAGCATGTGGAAGTGCATAC & Semi-quantitative RT-PCR of ACTIN1 \\
\hline JE75 & GATTCCCCTGCTTTTGTCTCCTCC & Semi-quantitative PCR of WRKY33 \\
\hline
\end{tabular}




\begin{tabular}{lll} 
JE76 & CAGCTTGATTGTTTGGACGAGTC & Semi-quantitative PCR of WRKY33 \\
\hline JE30 & GAAGAGTTTGCCGATGGAGG & Semi-quantitative PCR of WRKY53 \\
JE31 & CGAGGCTAATGGTGGTGTTC & Semi-quantitative PCR of WRKY53 \\
\hline DS64 & GACGCTTCATCTCGTCC & qRT PCR of UBIQUITIN5 \\
DS65 & GTAAACGTAGGTGAGTCCA & qRT PCR of UBIQUITIN5 \\
\hline JE73 & GGTCACAACAATCCGGAAGA & qRT PCR of WRKY33 \\
JE74 & GGAGAGACAAGAGAAGGAGAGA & qRT PCR of WRKY33 \\
\hline JE79 & TCACCGAGCGTACAACTTATTCC & qRT PCR of WRKY53 \\
JE80 & CGTTTATCGATGCCGGAGATT & qRT PCR of WRKY53 \\
\hline
\end{tabular}

\subsubsection{Enzymes}

\subsubsection{Restriction endonucleases}

Restriction endonucleases used in this work were obtained from Thermo Fisher Scientific (Waltham, USA) or New England Biolabs (Frankfurt (Main), Germany). The enzymes were used according to the manufacturer's instructions with the supplied 10x reaction buffers.

\subsubsection{Polymerases}

For standard polymerase chain reactions, homemade Taq polymerase was used. PCR products for cloning were amplified with Q5 High-Fidelity DNA Polymerase according to the manufacturer's instructions (New England Biolabs, Frankfurt/Main, Germany). cDNA was synthesized from total RNA with the RevertAid TM H Minus Reverse Transcriptase according to manufacturer's instructions (Thermo Fisher Scientific, Waltham, USA).

\subsubsection{Chemicals}

All chemicals used in this work were purchased from the following manufacturer: BioRad (Munich, Germany), Carbosynth (Compton, United Kingdom), Duchefa (Haarlem, The Netherlands), Invitrogen (Karlsruhe, Germany), WAKO Chemicals (Neuss, Germany), Megazyme (Bray, Ireland), Roth (Karlsruhe, Germany), Sigma-Aldrich (Munich, Germany), Thermo Fisher Scientific (Waltham, USA), PJK Biotech (Kleinblittersdorf, Germany) or VWR (Darmstadt, Germany). 


\subsubsection{Antibiotics}

Media for bacteria and yeast were supplemented with antibiotics to prevent contamination and select for transgenic organisms. The antibiotics used in this study are listed in Table 6.

Table 6. List of antibiotics used in this study.

\begin{tabular}{lll}
\hline Antibiotic & Stock solution & Final concentration \\
\hline \multirow{2}{*}{ Zeocin } & $100 \mathrm{mg} / \mathrm{ml}$ (provided by supplier) & $\begin{array}{l}25 \mu \mathrm{g} / \mathrm{ml}(\text { E.coli) } \\
100 / 500 \mu \mathrm{g} / \mathrm{ml} \text { (P. pastoris) }\end{array}$ \\
\hline
\end{tabular}




\subsubsection{Carbohydrates}

All cell-wall derived carbohydrates were orderd from Megazyme (Bray, Ireland) or Carbosynth (Compton, United Kingdom) and are listed in Table 7. All carbohydrates were dissolved in ultrapure water at a concentration of $10 \mathrm{mg} / \mathrm{ml}$ or $5 \mathrm{mg} / \mathrm{ml}$ according to the manufacturer's instructions. Oligosaccharides were stored at $-20^{\circ} \mathrm{C}$. Polysaccharides were stored at $4^{\circ} \mathrm{C}$. All information about the used carbohydrates are listed in Table 7 and were obtained from Megazyme (Bray, Ireland) or Carbosynth (Compton, United Kingdom).

Table 7. List of all carbohydrates used in this work.

\begin{tabular}{|c|c|c|c|c|}
\hline Name & Company & Source & $\begin{array}{l}\text { Purification Method } \\
\text { (Purity) }\end{array}$ & $\begin{array}{c}\text { Stock } \\
\text { concentration }\end{array}$ \\
\hline \multicolumn{5}{|l|}{ Polysaccharides } \\
\hline \multicolumn{5}{|c|}{$\beta-1,3-$ glucans/mixed linkage glucans } \\
\hline$\beta$-glucan & Megazyme & Barley & $\begin{array}{c}\text { GLC + Size Exclusion Chromatography } \\
(\sim 95 \%)\end{array}$ & $5 \mathrm{mg} / \mathrm{ml}$ \\
\hline Curdlan & Megazyme & $\begin{array}{c}\text { produced by Alcaligenes } \\
\text { faecalis var. myxogenes } \\
10 \mathrm{C} 3 \mathrm{~K} \\
\end{array}$ & - & $5 \mathrm{mg} / \mathrm{ml}$ \\
\hline Laminarin & $\begin{array}{c}\text { Santa Cruz } \\
\text { Biotechnology }\end{array}$ & Laminaria digitata & $-(\geq 96 \%)$ & $10 \mathrm{mg} / \mathrm{ml}$ \\
\hline Pachyman & Megazyme & $\begin{array}{l}\text { sclerotia of } \\
\text { Poria cocos }\end{array}$ & - & $5 \mathrm{mg} / \mathrm{ml}$ \\
\hline \multicolumn{5}{|l|}{ Hemicelluloses } \\
\hline Arabinoxylan & Megazyme & Wheat Flour & $\begin{array}{c}\text { GLC + Size Exclusion Chromatography } \\
\text { (Purity 95\%) }\end{array}$ & $10 \mathrm{mg} / \mathrm{ml}$ \\
\hline Galactomannan & Megazyme & Carob & $\begin{array}{c}\text { GLC + Size Exclusion Chromatography } \\
\text { (Purity }>94 \%)\end{array}$ & $10 \mathrm{mg} / \mathrm{ml}$ \\
\hline
\end{tabular}




\begin{tabular}{|c|c|c|c|c|}
\hline Glucomannan & Megazyme & Konjac & (Purity >98\%) & $10 \mathrm{mg} / \mathrm{ml}$ \\
\hline Mannan & Megazyme & - & (High Purity) & $10 \mathrm{mg} / \mathrm{ml}$ \\
\hline Xyloglucan & Megazyme & Tamarind & (Purity 95\%) & $10 \mathrm{mg} / \mathrm{ml}$ \\
\hline \multicolumn{5}{|l|}{ Pectic Components } \\
\hline Arabinan & Megazyme & Sugar Beet Pulp & $\begin{array}{c}\text { GLC } \\
\text { (Purity }>95 \%)\end{array}$ & $10 \mathrm{mg} / \mathrm{ml}$ \\
\hline Arabinogalactan & Megazyme & Larch Wood & $\begin{array}{l}\text { GLC + size exclusion chromatography } \\
\text { (Purity }>95 \%)\end{array}$ & $10 \mathrm{mg} / \mathrm{ml}$ \\
\hline Rhamnogalacturonan I & Megazyme & $\begin{array}{l}\text { pectic galactan from } \\
\text { potato fiber }\end{array}$ & $\begin{array}{c}\text { exhaustive hydrolysis of potato pectic } \\
\text { galactan and GLC } \\
\text { (High Purity) }\end{array}$ & $10 \mathrm{mg} / \mathrm{ml}$ \\
\hline Rhamnogalacturonan & Megazyme & soy bean pectin & $\begin{array}{c}\text { Exhaustive hydrolysis and GLC } \\
\text { (High Purity, }>97 \%)\end{array}$ & $10 \mathrm{mg} / \mathrm{ml}$ \\
\hline \multicolumn{5}{|l|}{ Oligosaccharides } \\
\hline \multicolumn{5}{|c|}{$\beta-1,3-$ glucans/mixed linkage glucans } \\
\hline 1,3:1,4-b-Glucotetraose (C) & Carbosynth & - & - & $10 \mathrm{mg} / \mathrm{ml}$ \\
\hline 1,3:1,4-b-Glucotriose (A) & Carbosynth & - & - & $10 \mathrm{mg} / \mathrm{ml}$ \\
\hline 1,3:1,4-b-Glucotriose (B) & Carbosynth & - & - & $10 \mathrm{mg} / \mathrm{ml}$ \\
\hline
\end{tabular}




\begin{tabular}{|c|c|c|c|c|}
\hline$\beta$-D-Cellotriosyl-glucose & Megazyme & Barley $\beta$-glucan & HPLC (>95\%) & $10 \mathrm{mg} / \mathrm{ml}$ \\
\hline $\begin{array}{c}\text { D-Cellobiosyl-cellobiose + } \\
\beta \text {-D-Glucosyl-cellotriose }\end{array}$ & Megazyme & Barley $\beta$-glucan & HPLC (>95\%) & $10 \mathrm{mg} / \mathrm{ml}$ \\
\hline Glucosyl-cellobiose & Megazyme & Barley $\beta$-glucan & HPLC (>95\%) & $10 \mathrm{mg} / \mathrm{ml}$ \\
\hline Cellobiosyl-glucose & Megazyme & Barley $\beta$-glucan & HPLC (>95\%) & $10 \mathrm{mg} / \mathrm{ml}$ \\
\hline Laminarihexaose & Megazyme & Curdlan & HPLC (>95\%) & $10 \mathrm{mg} / \mathrm{ml}$ \\
\hline Laminaripentaose & Megazyme & Curdlan & HPLC (>90 \%) & $10 \mathrm{mg} / \mathrm{ml}$ \\
\hline Laminaritetraose & Megazyme & Curdlan & HPLC (>95\%) & $10 \mathrm{mg} / \mathrm{ml}$ \\
\hline Laminaritriose & Megazyme & Curdlan & HPLC (>95\%) & $10 \mathrm{mg} / \mathrm{ml}$ \\
\hline Laminaribiose & Megazyme & Curdlan & HPLC (>95\%) & $10 \mathrm{mg} / \mathrm{ml}$ \\
\hline \multicolumn{5}{|l|}{ Cellulose Derivatives } \\
\hline Cellohexaose & Megazyme & - & HPLC + HPAEC-PAD (>95\%) & $10 \mathrm{mg} / \mathrm{ml}$ \\
\hline Isomaltotriose & Megazyme & - & HPLC + HPAEC-PAD (>98\%) & $10 \mathrm{mg} / \mathrm{ml}$ \\
\hline Maltotetraose & Megazyme & - & HPLC (>95\%) & $10 \mathrm{mg} / \mathrm{ml}$ \\
\hline \multicolumn{5}{|l|}{ Hemicelluloses } \\
\hline Arabinofuranosyl-xylotetraose & Megazyme & Arabinoxylan & HPLC (>95\%) & $10 \mathrm{mg} / \mathrm{ml}$ \\
\hline
\end{tabular}


Material and Methods

\begin{tabular}{|c|c|c|c|c|}
\hline $\begin{array}{l}\text { Arabinofuranosyl-xylotetraose } \\
(X A 3 X X / X A 2 X X) \text { mixture }\end{array}$ & Megazyme & Arabinoxylan & HPLC (>95\%) & $10 \mathrm{mg} / \mathrm{ml}$ \\
\hline Mannohexaose & Megazyme & Mannan & HPLC (>90\%) & $10 \mathrm{mg} / \mathrm{ml}$ \\
\hline$\alpha$-D-Galactosyl-mannopentaose & Megazyme & Galactomannan & HPLC (>95\%) & $10 \mathrm{mg} / \mathrm{ml}$ \\
\hline $\begin{array}{c}\text { 1,4- } \beta \text {-D-Glucosyl-D-Mannose + 1,4- } \beta \text { - } \\
\text { D-Mannobiose }\end{array}$ & Megazyme & Glucomannan & HPLC ( 96\%) & $10 \mathrm{mg} / \mathrm{ml}$ \\
\hline $\begin{array}{c}\text { 1,4- } \beta \text {-D-Glucosyl-D-Mannobiose + } \\
\text { 1,4- } \beta \text {-D-Cellobiosyl-D-Mannose }\end{array}$ & Megazyme & Glucomannan & HPLC (>90\%) & $10 \mathrm{mg} / \mathrm{ml}$ \\
\hline $\begin{array}{l}\text { Heptasaccharide (X3Glc4 - } \\
\text { borohydride reduced) }\end{array}$ & Megazyme & Xyloglucan & HPLC (>90\%) & $10 \mathrm{mg} / \mathrm{ml}$ \\
\hline Heptasaccharide (X3Glc4) & Megazyme & Xyloglucan & HPLC (>90\%) & $10 \mathrm{mg} / \mathrm{ml}$ \\
\hline $\begin{array}{l}\text { Higher Degree of Polymerisation } \\
\text { Xyloglucan Oligosaccharides }\end{array}$ & Megazyme & Xyloglucan & $(\sim 95 \%)$ & $10 \mathrm{mg} / \mathrm{ml}$ \\
\hline $\begin{array}{l}\text { Xyloglucan (hepta+octa+nona } \\
\text { saccharides) }\end{array}$ & Megazyme & Xyloglucan & HPLC ( 95 \%) & $10 \mathrm{mg} / \mathrm{ml}$ \\
\hline Xylohexaose & Megazyme & Xyloglucan & HPLC (>95\%) & $5 \mathrm{mg} / \mathrm{ml}$ \\
\hline \multicolumn{5}{|l|}{ Pectic Component } \\
\hline Arabino-octaose & Megazyme & Sugar beet & HPLC (>95\%) & $10 \mathrm{mg} / \mathrm{ml}$ \\
\hline \multicolumn{5}{|l|}{ Others } \\
\hline Kestopentaose & Megazyme & Fructan & HPLC (>95\%) & $10 \mathrm{mg} / \mathrm{ml}$ \\
\hline Verbascose & Megazyme & Bean extract & HPLC (>95\%) & $10 \mathrm{mg} / \mathrm{ml}$ \\
\hline
\end{tabular}




\subsubsection{Media}

All media listed in Table 8 were prepared with ultrapure water and autoclaved for 20 min at $121^{\circ} \mathrm{C}$. Antibiotics were added to the respective media after cooling down to $60^{\circ} \mathrm{C}$. Liquid and solid media were stored at room temperature.

Table 8. Media used in this study.

Media for growing $A$. thaliana seedlings in-vitro

\begin{tabular}{lll}
\hline $1 / 2 \mathrm{MS}+$ sucrose & Murashige and Skoog medium including & $2.2 \mathrm{~g} / \mathrm{l}$ \\
& Gamborg B5 vitamins & $10 \mathrm{~g} / \mathrm{l}$ \\
& Surcose & \\
& Adjust to $\mathrm{pH}=5.7$ with $\mathrm{KOH}$ & \\
& For aqueous agar plates, $2 \mathrm{~g} / \mathrm{l}$ plant agar were included \\
\hline
\end{tabular}

Media for growing Escherichia coli

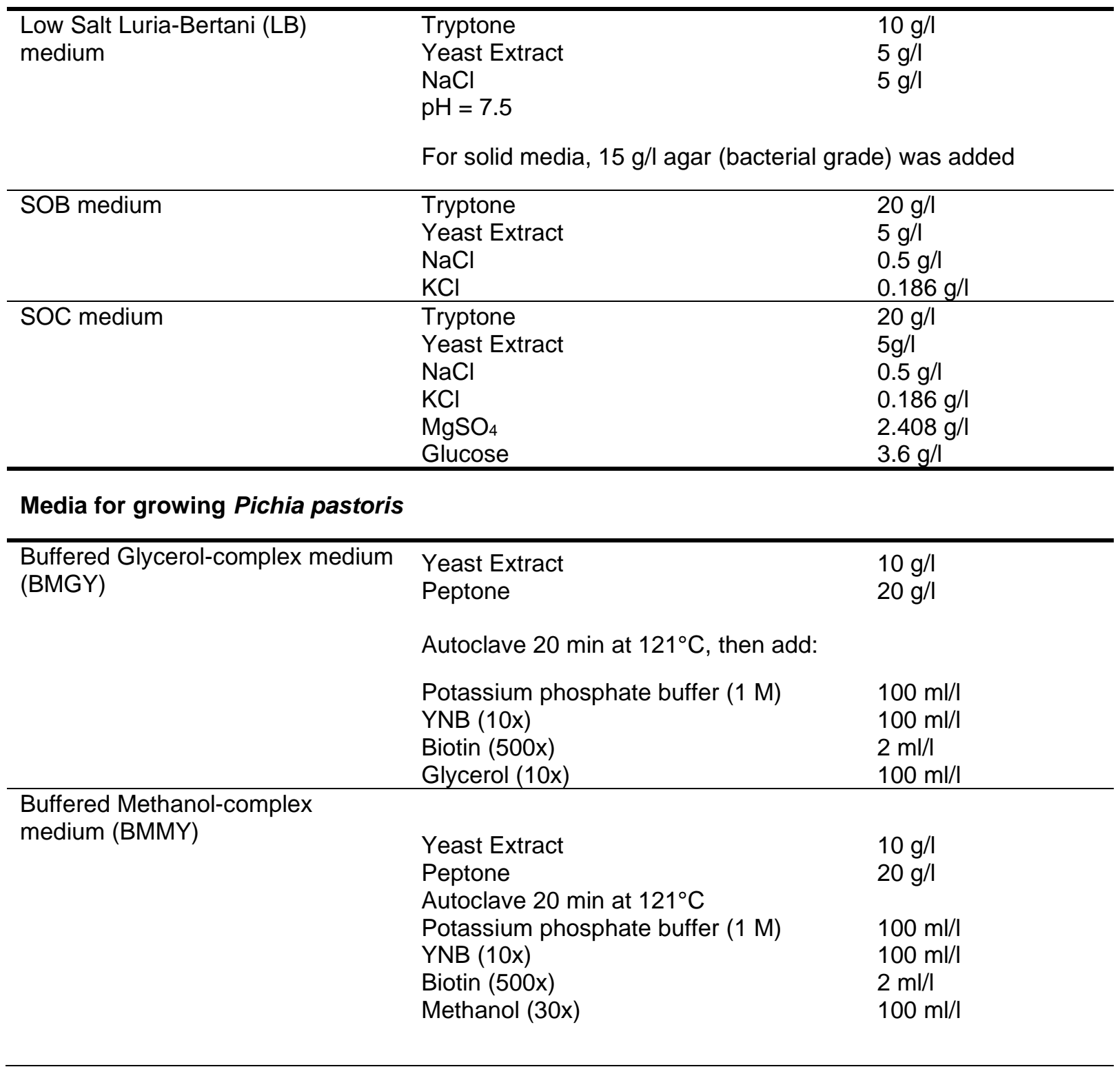




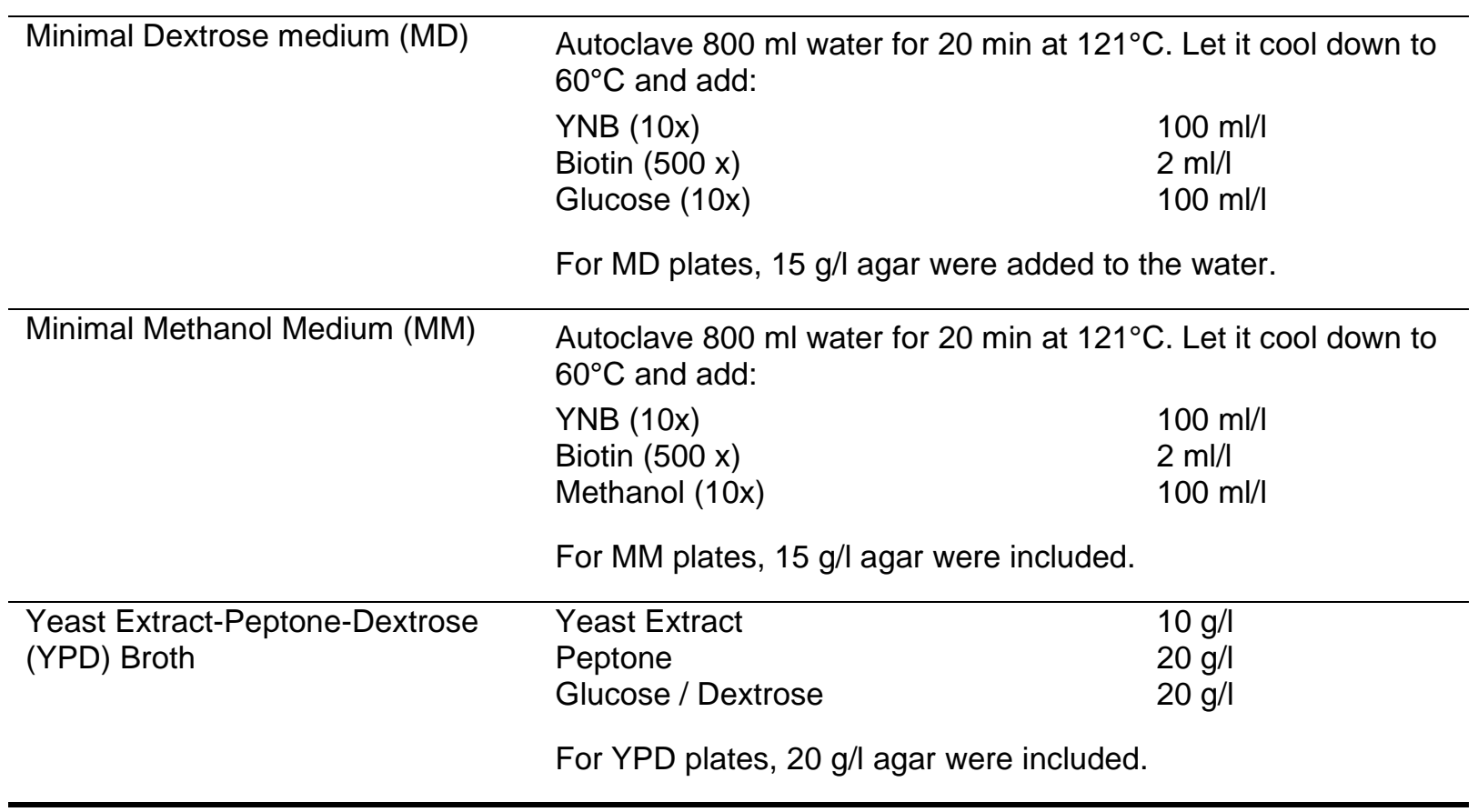

\section{Preparation of media for growth of Pichia pastoris}

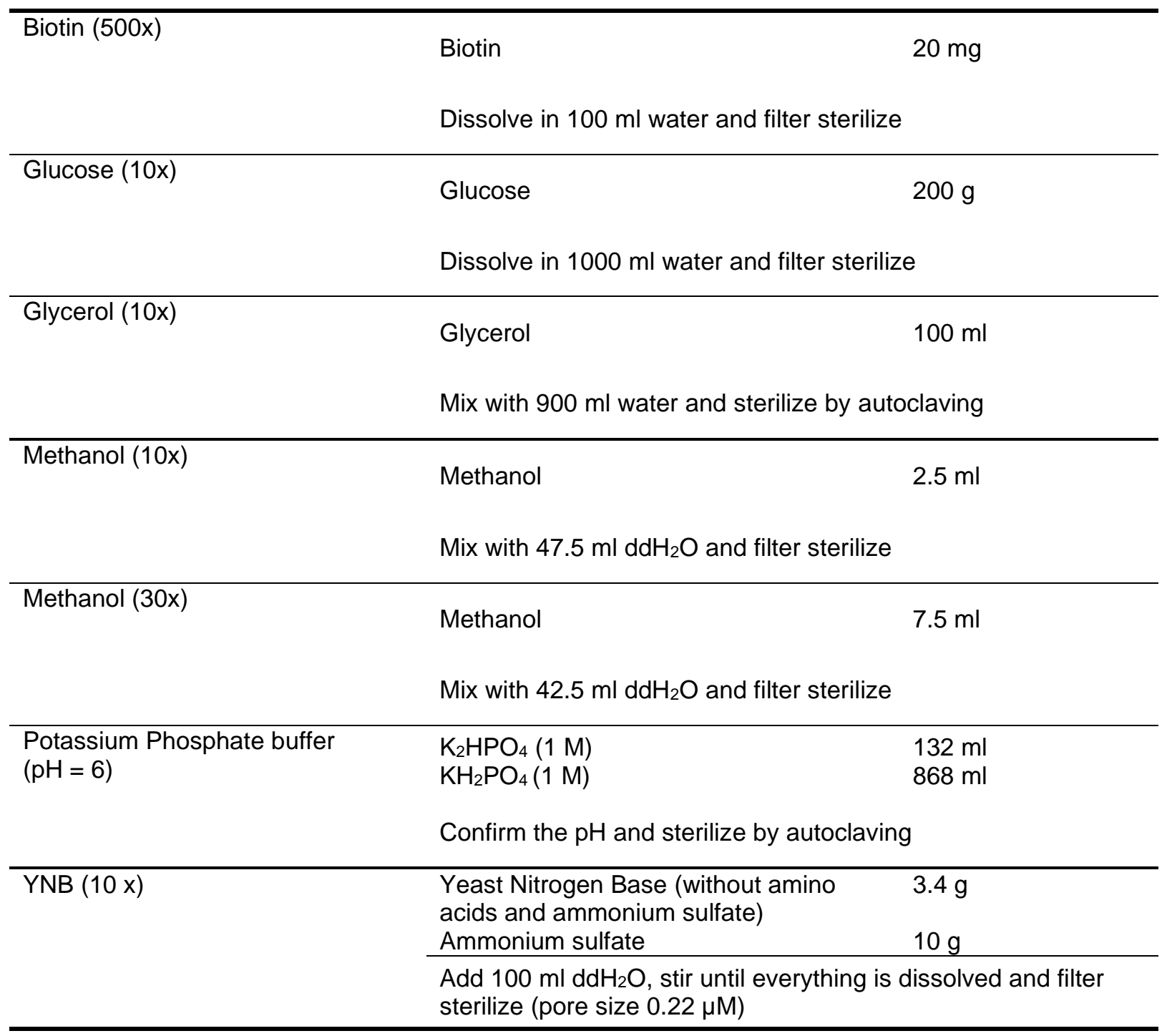




\subsubsection{Buffers and Solutions}

All buffers and solutions were prepared with ultrapure water and autoclaved for 20 min at $121^{\circ} \mathrm{C}$.

Buffers and solutions which were not autoclaved were sterilized by filtration with filters having a pore size of $0.2 \mu \mathrm{M}$. Solutions for the preparation of Pichia pastoris growth media were stored at $4^{\circ} \mathrm{C}$. Table 9 lists all buffers and solutions used in this work.

Table 9. List of buffers and solutions used in this work.

\section{Agarose Gel electrophoresis and PCR}

\begin{tabular}{lll}
\hline Agarose solution & Agarose & $1-3 \%$ \\
& TAE Buffer & $1 \mathrm{x}$ \\
\hline 6x DNA Loading Dye & Orange G & $0.25 \%(\mathrm{w} / \mathrm{v})$ \\
& Xylencyanol FF & $0.25 \% / \mathrm{w} / \mathrm{v})$ \\
& Glyercol & $30 \%(\mathrm{v} / \mathrm{v})$ \\
\hline 10x PCR reaction buffer for Taq polymerase & Tris base & $100 \mathrm{mM}$ \\
& $\mathrm{KCl}$ & $500 \mathrm{mM}$ \\
& $\mathrm{MgCl} 2$ & $15 \mathrm{mM}$ \\
& Triton X-100 & $1 \%(\mathrm{w} / \mathrm{v})$ \\
& $\mathrm{pH}=9($ with $\mathrm{KOH})$ & \\
& & $2 \mathrm{M}$ \\
& Tris base & $57.1 \mathrm{ml} / \mathrm{l}$ \\
& Glacial acetic acid & $100 \mathrm{ml} / \mathrm{l}$ \\
\hline \multirow{2}{*}{ G0x TAE buffer } & EDTA $(0.5 \mathrm{M}, \mathrm{pH}=8)$ & \\
& & \\
\hline \multirow{2}{*}{ DNA Extraction Buffer } & & $0.2 \mathrm{M}$ \\
& Tris- $\mathrm{HCl}(\mathrm{pH}=7.5)$ & $1.25 \mathrm{M}$ \\
& NaCl & $0.025 \mathrm{M}$ \\
& EDTA & $0.5 \%(\mathrm{w} / \mathrm{v})$ \\
\hline \multirow{2}{*}{ SDIants } & SDS & \\
\hline
\end{tabular}

\section{Protein Extraction from Plants}

\begin{tabular}{|c|c|c|}
\hline Barley extraction buffer & $\begin{array}{l}\text { Tris-HCl }(\mathrm{pH}=7.5) \\
\mathrm{NaCl} \\
\text { EGTA } \\
\beta \text {-glycerophosphat } \\
\mathrm{MgCl}_{2} \\
\mathrm{NaF} \\
\mathrm{Na}_{3} \mathrm{VO}_{4} \\
\mathrm{Na}_{2} \mathrm{MoO}_{4} \\
\text { DTT } \\
\text { Tween-20 } \\
\text { Protease Inhibitor } \\
\end{array}$ & $\begin{array}{l}50 \mathrm{mM} \\
100 \mathrm{mM} \\
20 \mathrm{mM} \\
30 \mathrm{mM} \\
20 \mathrm{mM} \\
4 \mathrm{mM} \\
4 \mathrm{mM} \\
4 \mathrm{mM} \\
10 \mathrm{mM} \\
0.2 \% \\
1: 1000 \\
\end{array}$ \\
\hline CERK1 extraction buffer & $\begin{array}{l}\text { Sucrose } \\
\text { HEPES-KOH }(\mathrm{pH}=7.5) \\
\text { Glycerol } \\
\mathrm{Na}_{2} \mathrm{MoO}_{4} \\
\mathrm{Na}_{4} \mathrm{P}_{2} \mathrm{O}_{7} \\
\mathrm{NaF} \\
\text { EDTA } \\
\text { DTT } \\
\text { Triton X-100 } \\
\text { Protease Inhibitor Cocktail }\end{array}$ & $\begin{array}{l}250 \mathrm{mM} \\
100 \mathrm{mM} \\
5 \%(\mathrm{v} / \mathrm{v}) \\
1 \mathrm{mM} \\
50 \mathrm{mM} \\
25 \mathrm{mM} \\
10 \mathrm{mM} \\
1 \mathrm{mM} \\
0.5 \%(\mathrm{w} / \mathrm{v}) \\
1: 100\end{array}$ \\
\hline
\end{tabular}




\begin{tabular}{|c|c|c|}
\hline \multirow[t]{3}{*}{ Protease Inhibitor Cocktail } & $\begin{array}{l}\text { 4-(2-aminoethyl) } \\
\text { benzenesulfonyl fluoride } \\
\text { hydrochloride (EABSF) }\end{array}$ & $1 \mathrm{~g}$ \\
\hline & $\begin{array}{l}\text { Bestatin hypochloride } \\
\text { Pepstatin A } \\
\text { Leupeptin hemisulfate } \\
\text { E-64 (trans-epoxysuccinyl-L- } \\
\text { leucylamido-(4- } \\
\text { guanidino)butane) }\end{array}$ & $\begin{array}{l}5 \mathrm{mg} \\
10 \mathrm{mg} \\
100 \mathrm{mg} \\
10 \mathrm{mg}\end{array}$ \\
\hline & $\begin{array}{l}\text { Phenantroline }(1,10- \\
\text { phenantroline monohydrate) } \\
\text { All components were dissolved } \\
\text { amount of DMSO and then col } \\
200 \mathrm{ml} \text { with } \mathrm{DMSO} \text {. The mixtu } \\
\mathrm{ml} \text { and stored at }-20^{\circ} \mathrm{C} \text {. }\end{array}$ & $\begin{array}{l}10 \mathrm{~g} \\
\text { eparately in a small } \\
\text { ined and filled up to } \\
\text { was aliquoted in } 2\end{array}$ \\
\hline
\end{tabular}

Buffers used for SDS PAGE and Immunoblot analysis

\begin{tabular}{|c|c|c|}
\hline 4x SDS Loading Buffer & $\begin{array}{l}\text { Tris-HCl }(\mathrm{pH}=6.8) \\
\text { DTT } \\
\text { SDS } \\
\text { Glycerol } \\
\text { Bromphenol Blue } \\
\text { Store at }-20^{\circ} \mathrm{C}\end{array}$ & $\begin{array}{l}200 \mathrm{mM} \\
400 \mathrm{mM} \\
8 \%(\mathrm{w} / \mathrm{v}) \\
40 \%(\mathrm{v} / \mathrm{v}) \\
0.1 \%(\mathrm{w} / \mathrm{v})\end{array}$ \\
\hline 10x SDS Running Buffer & $\begin{array}{l}\text { Glycine } \\
\text { Tris } \\
\text { SDS }\end{array}$ & $\begin{array}{l}2 \mathrm{M} \\
250 \mathrm{mM} \\
1 \%(\mathrm{w} / \mathrm{v})\end{array}$ \\
\hline 20x Transfer buffer & $\begin{array}{l}\text { Tris } \\
\text { Boric Acid } \\
\mathrm{pH}=8.3\end{array}$ & $\begin{array}{l}1 \mathrm{M} \\
1 \mathrm{M}\end{array}$ \\
\hline Alkaline Phosphatase (AP) buffer & $\begin{array}{l}\text { Tris-HCl, } \mathrm{pH}=9.5 \\
\mathrm{NaCl} \\
\mathrm{MgCl}_{2}\end{array}$ & $\begin{array}{l}100 \mathrm{mM} \\
100 \mathrm{mM} \\
50 \mathrm{mM}\end{array}$ \\
\hline $\begin{array}{l}\text { Coomassie Staining Solution for PVDF } \\
\text { membrane }\end{array}$ & $\begin{array}{l}\text { Ethanol } \\
\text { Acetic Acid } \\
\text { ddH }_{2} \mathrm{O} \\
\text { Coomassie R250 }\end{array}$ & $\begin{array}{l}300 \mathrm{ml} \\
100 \mathrm{ml} \\
300 \mathrm{ml} \\
0.05 \%\end{array}$ \\
\hline Destaining Solution for PVDF membrane & $\begin{array}{l}\text { Ethanol } \\
\text { Acetic Acid } \\
\mathrm{ddH}_{2} \mathrm{O}\end{array}$ & $\begin{array}{l}300 \mathrm{ml} \\
100 \mathrm{ml} \\
300 \mathrm{ml}\end{array}$ \\
\hline $20 \times$ TBS-T & $\begin{array}{l}\mathrm{NaCl} \\
\text { Tris-HCl }(\mathrm{pH}=8) \\
\text { Tween-20 }\end{array}$ & $\begin{array}{l}3 \mathrm{M} \\
200 \mathrm{mM} \\
1 \%\end{array}$ \\
\hline TBS-T + milk powder & $\begin{array}{l}\text { TBS-T } \\
\text { Skimmed Milk Powder }\end{array}$ & $40 \mathrm{~g} / \mathrm{l}$ \\
\hline
\end{tabular}

Preparation of chemically competent E.coli cells

\begin{tabular}{lll}
\hline CCMB80 & $\mathrm{KOAc}$ & $10 \mathrm{mM}$ \\
& $\mathrm{CaCl}_{2} \times 2 \mathrm{H}_{2} \mathrm{O}$ & $80 \mathrm{mM}$ \\
& $\mathrm{MnCL} 2 \times 4 \mathrm{H}_{2} \mathrm{O}$ & $20 \mathrm{mM}$ \\
& $\mathrm{MgCL} 2 \times 6 \mathrm{H}_{2} \mathrm{O}$ & $10 \mathrm{mM}$ \\
& Glycerol & $10 \%$
\end{tabular}


Solutions for purification of His-tagged proteins expressed in P. pastoris

\begin{tabular}{|c|c|c|}
\hline \multirow[t]{4}{*}{ Buffer A } & $\begin{array}{l}\mathrm{NaH}_{2} \mathrm{PO}_{4}(500 \mathrm{mM}) \\
\mathrm{Na}_{2} \mathrm{HPO}_{4}(500 \mathrm{mM})\end{array}$ & $\begin{array}{l}8 \mathrm{ml} \\
21 \mathrm{ml}\end{array}$ \\
\hline & \multicolumn{2}{|c|}{ Add $\mathrm{H}_{2} \mathrm{O}$ to $500 \mathrm{ml}$, adjust $\mathrm{pH}$ to 7.5 , then add: } \\
\hline & $\begin{array}{l}\mathrm{NaCl} \\
\text { Imidazole }\end{array}$ & $\begin{array}{l}8.73 \mathrm{~g} \\
0.34 \mathrm{~g}\end{array}$ \\
\hline & Filter sterile & \\
\hline \multirow[t]{4}{*}{ Buffer C } & $\begin{array}{l}\mathrm{NaH}_{2} \mathrm{PO}_{4}(500 \mathrm{mM}) \\
\mathrm{Na}_{2} \mathrm{HPO}_{4}(500 \mathrm{mM})\end{array}$ & $\begin{array}{l}8 \mathrm{ml} \\
21 \mathrm{ml}\end{array}$ \\
\hline & \multicolumn{2}{|c|}{ Add $\mathrm{H}_{2} \mathrm{O}$ to $500 \mathrm{ml}$, adjust $\mathrm{pH}$ to 7.5 , then add: } \\
\hline & $\begin{array}{l}\mathrm{NaCl} \\
\text { Imidazole }\end{array}$ & $\begin{array}{l}8.73 \mathrm{~g} \\
17.11 \mathrm{~g}\end{array}$ \\
\hline & \multicolumn{2}{|l|}{ Filter sterile } \\
\hline SEC Buffer & $\begin{array}{l}\text { MOPS } \\
\text { EDTA } \\
\text { pH }=7.5\end{array}$ & $\begin{array}{l}20 \mathrm{mM} \\
1 \mathrm{mM}\end{array}$ \\
\hline \multicolumn{3}{|c|}{ Thin Layer Chromatography (TLC) } \\
\hline \multirow[t]{2}{*}{ TLC running buffer } & $\begin{array}{l}\text { Isopropanol } \\
\text { Ethylacetate }\end{array}$ & $\begin{array}{l}200 \mathrm{ml} \\
200 \mathrm{ml}\end{array}$ \\
\hline & \multicolumn{2}{|c|}{ Fill up to $500 \mathrm{ml}$ with $\mathrm{ddH}_{2} \mathrm{O}$} \\
\hline TLC staining solution & $\begin{array}{l}\text { Sulfuric acid } \\
\text { In methanol }\end{array}$ & $10 \%$ \\
\hline \multicolumn{3}{|l|}{ ROS Burst Assay } \\
\hline L-012 & $\begin{array}{l}\text { Horseradish Peroxidase } \\
\mathrm{L}-012 \\
\text { In } \mathrm{ddH}_{2} \mathrm{O}\end{array}$ & $\begin{array}{l}10 \mu \mathrm{g} / \mathrm{ml} \\
100 \mu \mathrm{M}\end{array}$ \\
\hline \multicolumn{3}{|l|}{ Calcium Assay } \\
\hline Coelenterazine working solution & $\begin{array}{l}\text { Coelenterazine } \\
\text { In methanol }\end{array}$ & $40 \mu \mathrm{M}$ \\
\hline Discharge Solution & $\begin{array}{l}\mathrm{CaCl}_{2} \\
\mathrm{EtOH} \\
\text { In ddH }\end{array}$ & $\begin{array}{l}2 \mathrm{M} \\
20 \%\end{array}$ \\
\hline
\end{tabular}




\subsubsection{Antibodies}

Primary and secondary antibodies that were used in this work are listed in Table 10. The primary antibody a-pMAPK Phospho p44/42 was stored at $-20^{\circ} \mathrm{C}$, whereas all other antibodies were aliquoted and stored at $-80^{\circ} \mathrm{C}$ for long term storage. Aliquots of antibodies in use were kept at $4^{\circ} \mathrm{C}$ or $-20^{\circ} \mathrm{C}$.

Table 10. List of antibodies used in this study.

\begin{tabular}{ccc}
\hline Primary Antibody & Produced in (organism) & Company \\
\hline $\begin{array}{c}\text { a-pMAPK } \\
\begin{array}{c}\text { Phospho p44/42 (Erk1/2) } \\
\text { (Thr202/Tyr204) } \\
\text { (used: } 1: 5000)\end{array}\end{array}$ & Rabbit, polyclonal & $\begin{array}{c}\text { Cell Signaling Technology, } \\
\text { Danvers, MA, USA }\end{array}$ \\
\hline Secondary Antibody & Produced in (organism) & Company \\
\hline $\begin{array}{c}\text { Anti-rabbit IgG AP conjugate } \\
\text { (used: } 1: 5000)\end{array}$ & Goat, polyclonal & $\begin{array}{c}\text { Sigma-Aldrich, Munich, } \\
\text { Germany }\end{array}$ \\
\hline
\end{tabular}

\subsubsection{Devices}

Table 11. Devices used in this study.

\begin{tabular}{|c|c|c|}
\hline Device & Model & Manufacturer \\
\hline Blot Imaging System & ChemiDoc Touch & Bio-Rad (Hercules, CA, USA) \\
\hline Bunsenburner & Phoenix eco & $\begin{array}{l}\text { Schuett biotect (Göttingen, } \\
\text { Germany) }\end{array}$ \\
\hline Centrifuges & Pico 21 & $\begin{array}{l}\text { Thermo Fisher Scientific } \\
\text { (Langenselbold, Germany) }\end{array}$ \\
\hline Clean Bench & Hera safe & $\begin{array}{l}\text { Thermo Fisher Scientific } \\
\text { (Langenselbold, Germany) }\end{array}$ \\
\hline Computer & OptiPlex5040 & Dell (Halle (Saale), Germany) \\
\hline Dewer & - & $\begin{array}{l}\text { Nalgene (Rochester, NY, } \\
\text { USA) }\end{array}$ \\
\hline Freezer $\left(-20^{\circ} \mathrm{C}\right)$ & Mediline & $\begin{array}{l}\text { Liebherr (Kirchdorf an der } \\
\text { Iller, Germany) }\end{array}$ \\
\hline Freezer $\left(-80^{\circ} \mathrm{C}\right)$ & Hera freeze & $\begin{array}{l}\text { Thermo Fisher Scientific } \\
\text { (Langenselbold, Germany) }\end{array}$ \\
\hline Gel documentation system & GenoPlex & VWR (Hannover, Germany) \\
\hline $\begin{array}{l}\text { Gel electrophoresis } \\
\text { equipment }\end{array}$ & - & BioRad (Hercules, CA, USA) \\
\hline Gel running chamber & Sub Cell $\circledast \mathrm{GT}$ & BioRad (Hercules, CA, USA) \\
\hline Growth Chamber & - & $\begin{array}{l}\text { Johnson Controls } \\
\text { (Milwaukee, WI, USA) }\end{array}$ \\
\hline Heating Plate & OTS40 & Medite (Burgdorf, Germany) \\
\hline
\end{tabular}




\begin{tabular}{|c|c|c|}
\hline HPAEC-PAD & $\begin{array}{l}\text { Dionex ICS-5000 with an AS-AP } \\
\text { auto-sampler and a temperature- } \\
\text { controlled sample tray run }\end{array}$ & Thermo Fisher Scientific \\
\hline $\begin{array}{l}\text { Analytical Column for } \\
\text { HPAEC-PAD }\end{array}$ & $\begin{array}{l}3 \text { * } 250 \mathrm{~mm} \text { Dionex CarboPac } \\
\text { PA200 }\end{array}$ & Thermo Fisher Scientific \\
\hline $\begin{array}{l}\text { Guard Column for HPAEC- } \\
\text { PAD }\end{array}$ & $3 \times 50 \mathrm{~mm}$ guard column & Thermo Fisher Scientific \\
\hline Ice Machine & - & $\begin{array}{l}\text { Ziegra (Isernhagen, } \\
\text { Germany) }\end{array}$ \\
\hline Magnetic stirrer & RH basic 2 IKAMAG & IKA (Staufen, Germany) \\
\hline Mass Spectrometry & $\begin{array}{l}\text { Waters } \AA \text { Xevo® QTOF with a } \\
\text { nanoACQUITY UPLC system }\end{array}$ & $\begin{array}{l}\text { Waters (Milford, } \\
\text { Massachusetts, USA) }\end{array}$ \\
\hline MALDI-TOF & $\begin{array}{l}\text { Autoflex MALDI-TOF equipped with } \\
\text { Smartbeam-II } 355 \mathrm{~nm} \text { laser system }\end{array}$ & $\begin{array}{l}\text { Bruker (Billerica, } \\
\text { Massachusetts, USA) }\end{array}$ \\
\hline PCR Cycler & MyCycler & BioRad (Hercules, CA, USA) \\
\hline $\mathrm{pH}$ Meter & Inolab $\AA$ & WTW (Weilheim, Germany) \\
\hline Photometer & Infinite M200 & $\begin{array}{l}\text { Tecan (Wiesbaden, } \\
\text { Germany) }\end{array}$ \\
\hline Photometer II & WPA Biowave II & Biochrom (Cambridge, UK) \\
\hline Pipet & Pipetman & $\begin{array}{l}\text { Gilson (Limburg-Offheim, } \\
\text { Germany) }\end{array}$ \\
\hline qRT-PCR Cycler & C199 Touch with CFX96 system & BioRad (Hercules, CA, USA) \\
\hline Refrigerator & Mediline & $\begin{array}{l}\text { Liebherr (Kirchdorf an der } \\
\text { Iller, Germany) }\end{array}$ \\
\hline Steam sterilizer & Varioklav 75S / 135S & $\begin{array}{l}\text { Thermo Fisher Scientific } \\
\text { (Langenselbold, Germany) }\end{array}$ \\
\hline Thermomixer & Compact / Comfort & $\begin{array}{l}\text { Eppendorf (Hamburg, } \\
\text { Germany) }\end{array}$ \\
\hline Vortexter & VF2 & IKA (Staufen, Germany) \\
\hline Water filter system & Arium $\AA^{\circledR} 611 \mathrm{DI}$ & $\begin{array}{l}\text { Sartorius (Göttingen, } \\
\text { Germany) }\end{array}$ \\
\hline
\end{tabular}

\subsubsection{Software}

Table 12. Software that was used in this study.

\begin{tabular}{ll}
\hline Software & Source \\
\hline Acrobat Reader & $\begin{array}{l}\text { http://get.adobe.com/uk/reader/ } \\
\text { (Adobe Systems Inc., San José, CA; USA) }\end{array}$ \\
\hline Adobe Illustrator CS5 v 15.0.0 & (Adobe Systems Inc., San José, CA; USA) \\
\hline Adobe Photoshop CS5 v 12.0 & (Adobe Systems Inc., San José, CA; USA) \\
\hline Bio-Rad CFX Manager 3.0 & (Bio-Rad Laboratories, Hercules, CA, USA) \\
\hline Chromeleon 7 & ThermoFisher Scientific \\
\hline
\end{tabular}




\begin{tabular}{ll}
\hline Clone Manager Professional Suite v 8 & $\begin{array}{l}\text { http://www.scied.com/pr_cmpro.htm } \\
\text { (Sci-Ed Software, Denver, CO, USA) }\end{array}$ \\
\hline dBCan & $\begin{array}{l}\text { http://bcb.unl.edu/dbCAN2 } \\
\text { (Yin et al., 2012) }\end{array}$ \\
\hline Geneious & $\begin{array}{l}\text { http://www.geneious.com/ } \\
\text { (Biomatters Ltd., Auckland, New Zealand) }\end{array}$ \\
\hline NetNGlyc & http://www.cbs.dtu.dk/services/NetNGlyc/ \\
\hline NetPhos & http://www.cbs.dtu.dk/services/NetPhos \\
\hline Office 2010 & (Microsoft, redmont, WA, USA) \\
\hline Origin Pro graphing software & \begin{tabular}{l} 
OriginLab (Washington, USA) \\
\hline SecretomeP 2.0
\end{tabular} \\
\hline SignalP 3.0 & $\begin{array}{l}\text { http://www.cbs.dtu.dk/services/SecretomeP/; } \\
\text { (Bendtsen et al., 2004a) }\end{array}$ \\
\hline
\end{tabular}




\subsection{Methods}

\subsubsection{Methods for working with plants}

\subsubsection{Arabidopsis thaliana}

\subsection{Sterilization}

For growth of $A$. thaliana on soil, the seeds were frozen to kill off potential pathogens. To this end, seeds were put doubly into plastic backs and incubated for $48 \mathrm{~h}$ at $-20^{\circ} \mathrm{C}$. Before sowing, the seeds were warmed up again at room temperature.

For growth of $A$. thaliana in-vitro, seeds were sterilized with ethanol. Therefore, seeds were transferred into $1.5 \mathrm{ml}$ tubes and washed three times for 2 min with $70 \% \mathrm{EtOH}$ and $0.05 \%$ Tween-20. To ensure equal washing of the seeds, the tubes were incubated on a stirring wheel. Next, the seeds were washed twice with $100 \% \mathrm{EtOH}$ for $1 \mathrm{~min}$. A sterile filter paper was put into the lid of a petri dish. For drying, the seeds together with the $\mathrm{EtOH}$ were poured onto the paper. The seeds were used further when the EtOH was evaporated.

The procedure was performed under a sterile bench.

\subsection{Plant growth conditions for growth on soil}

The sterilized seeds were sown on damp soil (Frühstorfer Erde, Type T, Archut) that was steamed $30 \mathrm{~min}$ at $90^{\circ} \mathrm{C}$. To enhance humidity and promote germination, a transparent lid was used to cover the pots. The pots were then brought to growth chambers (Johnson Controls, Milwaukee, WI, USA) with short day conditions ( $8 \mathrm{~h}$ light, $22^{\circ} \mathrm{C}, 140 \mathrm{~mol} \mathrm{~m}^{-2} \mathrm{sec}^{-1}, 65 \%$ relative humidity. The lids were removed when the seeds germinated. To induce flowering and setting of seeds, plants were transferred to long day conditions (16h light, $26^{\circ} \mathrm{C}, 200 \mathrm{~m}^{-2} \mathrm{sec}^{-1}, 65 \%$ relative humidity).

\subsection{Plant growth conditions for in-vitro culture}

Arabidopsis seedlings used for calcium assays were grown in-vitro in petri dishes. For this purpose, seeds sterilized with ethanol were sown on $1 / 2 M S+$ sucrose semi-solid agar and grown for $7-9 d$ in a growth cabinet (CLF Plant Climatics, Wertingen, Germany) with short day conditions (12h light, 12h darkness). The 7-9-day old seedlings were then transferred into 96-well plates. For the measurement, the seedlings were covered with $75 \mu \mathrm{l}$ water and $25 \mu \mathrm{l}$ coelenterazine and incubated overnight at room temperature in the dark.

Arabidopsis seedlings used for Immunoblot or gene expression analysis were grown in-vitro in 24-well plates. Therefore, seeds sterilized with ethanol were sown on $1 / 2 M S+$ sucrose aqueous agar. The seeds were allowed to germinate and grow for $7 \mathrm{~d}$ in a growth cabinet (CLF Plant Climatics, Wertingen, Germany) with short day conditions (12h light, $12 \mathrm{~h}$ darkness). The $7 \mathrm{~d}$ old seedlings were then transferred to 24-well plates containing $500 \mu \mathrm{L} 1 / 2 \mathrm{MS}+$ sucrose medium and allowed to grow in a growth cabinet (CLF Plant Climatics, Wertingen, Germany) with short day conditions (12h light, 12h darkness). Two seedlings were transferred into one well. After $13 d$ the medium was replaced with $500 \mu \mathrm{L}$ new $1 / 2 \mathrm{MS}+$ sucrose medium to ensure equal amounts of medium in each well. The next day (day 14$)$, the seedlings were treated with chitin $\left(10 \mu \mathrm{g} \mathrm{ml}^{-1}\right)$, flg22 $(50 \mathrm{nM})$, oligogalacturonides $(1,5$, 
$10 \mu \mathrm{g} \mathrm{ml}^{-1}$ ) and medium as control as well as with different cell-wall derived oligo- and polysaccharides $\left(10 \mu \mathrm{g} \mathrm{ml}^{-1}\right)$.

\subsubsection{Hordeum vulgare}

\subsection{Sterilization}

For growth of Hordeum vulgare on soil, the seeds were frozen to kill of potential pathogens. Therefore, seeds were transferred into a plastic bag and incubated overnight at $-20^{\circ} \mathrm{C}$. Before sowing, the seeds were allowed to warm up at room temperature.

\subsection{Plant growth conditions for growth on soil}

The surface sterilized seeds were sown and lightly covered with soil (Frühstorfer Erde, Type T, Archut) that was steamed beforehand for $30 \mathrm{~min}$ at $90^{\circ} \mathrm{C}$. To promote germination, the pots were covered with a transparent lid and the pots were brought to a growth chamber (Johnson Controls, Milwaukee, WI, USA) with long day conditions (16h light, $26^{\circ} \mathrm{C}, 200 \mathrm{~m}^{-2} \mathrm{sec}^{-1}, 65 \%$ relative humidity). Upon germination, the lid was removed.

\subsection{Treatment of $H$. vulgare for immunoblot analysis}

For analyzing the activation of MAPK in $H$. vulgare upon MAMP or carbohydrate treatment, 12-14 leaf discs of $4 \mathrm{~mm}$ diameter were harvested from second leaves of $14 \mathrm{~d}$ old $H$. vulgare plants. The

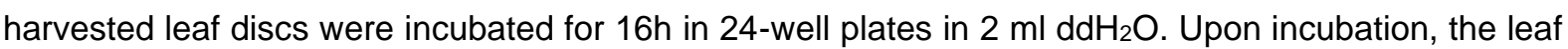
discs were transferred to a new 24-well plate with each well containing $500 \mu \mathrm{ldd}_{2} \mathrm{O}$. Then, the leaf

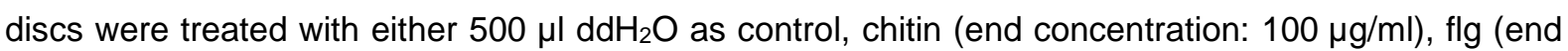
concentration: $50 \mathrm{nM}$ ) or carbohydrates (end concentration: $100 \mu \mathrm{g} / \mathrm{ml}$ ). $H$. vulgare was only treated with carbohydrates belonging to the group of mixed linkage glucans (see Table 7).

\subsubsection{Methods for working with Escherichia coli}

\subsubsection{Growth conditions for E.coli}

E.coli cells were cultivated on solid LB plates or in liquid LB medium with the respective antibiotics as selective markers. Single colonies from LB plates were used to inoculate liquid medium.

LB plates with E.coli cells were incubated at $37^{\circ} \mathrm{C}$ overnight. Liquid cultures were grown at $37^{\circ} \mathrm{C}$ at $220 \mathrm{rpm}$.

\subsubsection{Preparation of competent $E$. coli cells}

One single colony of $E$. coli TOP10 F' cells was inoculated in $5 \mathrm{ml}$ low salt LB medium and incubated at $37^{\circ} \mathrm{C}$ overnight while shaking. The next day, the $5 \mathrm{ml}$ culture was transferred into $500 \mathrm{ml} \mathrm{SOB}$ medium and incubated at $37^{\circ} \mathrm{C}$ while shaking to an $\mathrm{OD}_{600}$ of 0.4 . The culture was incubated for $30 \mathrm{~min}$ on ice and then centrifuged at $3000 \mathrm{rpm}$ at $4^{\circ} \mathrm{C}$ for $10 \mathrm{~min}$. The supernatant was discarded and the pellet was dissolved in $80 \mathrm{ml}$ ice-cold CCMB80. After incubation of the solution for $20 \mathrm{~min}$ on ice, the solution was centrifuged at $4^{\circ} \mathrm{C}$ for $10 \mathrm{~min}$ at $3000 \mathrm{rpm}$. The supernatant was again discarded and the pellet resuspended in $10 \mathrm{ml}$ ice-cold CCMB80. Next, the $\mathrm{OD}_{600}$ of a mixture of $200 \mu \mathrm{l} \mathrm{SOC}$ and $50 \mu \mathrm{l}$ 
of the resuspended cells was tested and the $\mathrm{OD}_{600}$ was adjusted to 1.0-1.5 with ice-cold CCMB80. Finally, aliquots of $100 \mu \mathrm{l}$ cells were prepared and frozen in liquid nitrogen. The cells were then stored at $-80^{\circ} \mathrm{C}$.

\subsubsection{Transformation of $E$. coli}

An aliquot of competent $E$. coli cells was thawed on ice. $2 \mu \mathrm{L}$ of the Gibson Assembly mix were added to the cells and mixed. The cells were incubated on ice for $30 \mathrm{~min}$, followed by a heatshock at $42^{\circ} \mathrm{C}$ for $45 \mathrm{sec}$. After the heatshock, the cells were incubated for $2 \mathrm{~min}$ on ice and then, $900 \mu \mathrm{L} \mathrm{SOC}$ or low salt LB medium were added to the cells. Upon incubation for $60 \mathrm{~min}$ at $37^{\circ} \mathrm{C}$ while shaking, the cells were plates on low salt LB medium containing the respective antibiotics.

\subsubsection{Methods for working with Pichia pastoris}

\subsubsection{Growth conditions for $\boldsymbol{P}$. pastoris}

P. pastoris cells were cultivated on solid YPD plates in or liquid YPD medium with the respective antibiotic as selective marker. For determining the Mut phenotype, $P$. pastoris cells were grown on solid MD and MM plates. For expression studies, $P$. pastoris cells were grown in liquid BMGY and BMMY medium. Single colonies from YPD plates were used to inoculate liquid YPD or BMGY medium.

YPD plates with $P$. pastoris cells were incubated at $28^{\circ} \mathrm{C}-30^{\circ} \mathrm{C}$ for $2-3 \mathrm{~d}$. Liquid cultures were grown at $30^{\circ} \mathrm{C}$ at $160 \mathrm{rpm}$. For expression studies, cultures were grown at either $16^{\circ} \mathrm{C}$ or $25^{\circ} \mathrm{C}$ at $160 \mathrm{rpm}$.

\subsubsection{Preparation of competent $\boldsymbol{P}$. pastoris cells}

In the morning, a single colony of $P$. pastoris X-33 was inoculated two times in $2.5 \mathrm{ml}$ YPD in a sterile $50 \mathrm{ml}$ tube. The cultures were grown over the day at $30^{\circ} \mathrm{C}$ at $160 \mathrm{rpm}$. In the afternoon, $500 \mu \mathrm{L}$ of the pre-culture were inoculated in $250 \mathrm{ml} \mathrm{YPD}$ and incubated overnight at $30^{\circ} \mathrm{C}$ at $160 \mathrm{rpm}$ to an $\mathrm{OD}_{600}$ of 1.2-1.5. Next, the culture was centrifuged at $4^{\circ} \mathrm{C}$ at $2.000 \mathrm{~g}$ for $5 \mathrm{~min}$. The supernatant was discarded and the pellet was gently resuspended in $100 \mathrm{ml}$ YPD with $20 \mathrm{ml} \mathrm{HEPES} \mathrm{(pH=8).} 2.5 \mathrm{ml}$ freshly prepared DTT $(1 \mathrm{M})$ were gently mixed to the solution and the solution was incubated for $15 \mathrm{~min}$ at $30^{\circ} \mathrm{C}$ without shaking. The volume of the solution was adjusted to $400 \mathrm{ml}$ with ice-cold sterile water. Again, the solution was centrifuged for $5 \mathrm{~min}$ at $2000 \mathrm{~g}$ at $4^{\circ} \mathrm{C}$. The supernatant was discarded and the pellet was resuspended in $250 \mathrm{ml}$ ice-cold sterile water. After centrifugation of the solution for 5 min at $2000 \mathrm{~g}$ at $4^{\circ} \mathrm{C}$, the supernatant was discarded and the pellet was resuspended in $20 \mathrm{ml}$ ice-cold sorbitol $(1 \mathrm{M})$. The solution was transferred into a $50 \mathrm{ml}$ tube and centrifuged for $5 \mathrm{~min}$ at $4^{\circ} \mathrm{C}$ for $5 \mathrm{~min}$ at $2000 \mathrm{~g}$. Again the supernatant was discarded and the pellet was resuspended in $500 \mu \mathrm{l}$ cold Sorbitol $(1 \mathrm{M})$. Finally, aliquots of $80 \mu \mathrm{l}$ cells were prepared. The cells could either be used directly or can be stored for $1-2$ weeks at $-80^{\circ} \mathrm{C}$.

\subsubsection{Transformation of competent $\boldsymbol{P}$. pastoris cells}

Prior to transformation of $P$. pastoris cells, the respective plasmids had to be linearized (see 2.2.4.8). $10 \mu \mathrm{L}$ of the linearized plasmid were mixed with $80 \mu \mathrm{l}$ competent $P$. pastoris cells and transferred into 
an ice-cold $0.2 \mathrm{~cm}$ electroporation cuvette. Upon incubation of the cuvette for $5 \mathrm{~min}$ on ice, the cells were pulsed with a Micropulser (BioRad, Munich, Germany) according to the manufacturer's instructions. $1 \mathrm{ml}$ ice-cold sorbitol $(1 \mathrm{M})$ was added to the cells directly after the electro pulse. Then, the cells were transferred into a sterile $15 \mathrm{ml}$ tube and incubated at $30^{\circ} \mathrm{C}$ for $1-2 \mathrm{~h}$ without shaking. After incubation, $80 \mu \mathrm{l}, 100 \mu \mathrm{l}$ and $150 \mu \mathrm{l}$ cells were plated on separate YPD plates containing either $100 \mu \mathrm{g} / \mathrm{ml}$ or $500 \mu \mathrm{g} / \mathrm{ml}$ Zeocin.

\subsubsection{Determination of the Mut phenotype}

$P$. pastoris $\mathrm{X}-33$ has a Mut ${ }^{+}$phenotype. However, during transformation of $P$. pastoris $\mathrm{X}-33$, the recombination could occur at the 3'AOX1 region thereby disrupting the wild-type AOX1 gene. Thus, transformants having a Mut ${ }^{S}$ phenotype can be generated. To test the Mut phenotype, growth of $P$. pastoris transformants was tested on MM and MD plates. To this end, $10-20$ transformants were picked and streaked out on MM first and then onto MM plates and incubated for $2 \mathrm{~d}$ at $30^{\circ} \mathrm{C}$.

Upon confirming the Mut phenotype, the $P$. pastoris transformants were for tested for the expression of the gene of interest via small scale expression.

\subsubsection{Molecular biology methods}

\subsubsection{Isolation of genomic DNA of $A$. thaliana}

$300 \mu \mathrm{L}$ DNA extraction buffer were added to a small Arabidopsis leaf in a $1.5 \mathrm{ml}$ tube. The leaf was grounded with a plastic pistil and incubated for $5 \mathrm{~min}$ at RT at $800 \mathrm{rpm}$ on a shaker. Afterwards, the samples were centrifuged for $5 \mathrm{~min}$ at maximum $13.000 \mathrm{rpm}$ at RT. $240 \mu \mathrm{l}$ of the supernatant were transferred to a new tube and $300 \mu \mathrm{l}$ isopropanol were added. Upon incubating the samples on a shaker for $5 \mathrm{~min}$ at RT, the samples were centrifuged for $10 \mathrm{~min}$ at RT at $13.000 \mathrm{rpm}$ and the supernatant was discarded. To remove the remaining supernatant, the samples were again centrifuged for $1 \mathrm{~min}$ at RT at $13.000 \mathrm{rpm}$. Afterwards, the DNA pellet was air-dried and finally

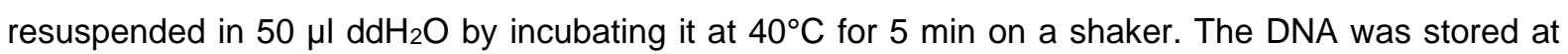
$-20^{\circ} \mathrm{C}$ or directly used for PCR.

\subsubsection{Isolation of plasmid DNA from $E$. coli}

\subsection{Small Scale plasmid isolation}

For small scale plasmid isolation, $5 \mathrm{ml}$ overnight culture was spun down and used for plasmid isolation with the Presto ${ }^{\mathrm{TM}}$ Mini Plasmid Kit (Geneaid Biotech Ltd, Taipei, Taiwan) according to the manufacturer's instructions.

\subsection{Medium Scale plasmid isolation}

To purify a higher amount of plasmid, $100 \mathrm{ml}$ culture was centrifuged and used for plasmid isolation using the Plasmid Mini, Midi, and Maxi Kit (QIAgen, Hilden, Germany) according to the manufacturer's instructions. 


\subsubsection{Polymerase Chain Reaction (PCR) for cloning}

For generating DNA used for cloning, the Q5 High-Fidelity DNA polymerase (New England Biolabs, Frankfurt/Main, Germany) was used.

PCR Mix for one reaction

$\begin{array}{ll}\text { 5x Q5 reaction buffer } & 10 \mu \mathrm{l} \\ \text { dNTPs }(10 \mathrm{mM}) & 1 \mu \mathrm{l} \\ \text { Primer } 1(10 \mu \mathrm{M}) & 2.5 \mu \mathrm{l} \\ \text { Primer } 2(10 \mu \mathrm{M}) & 2.4 \mu \mathrm{l} \\ \text { Q5 Polymerase } & 0.5 \mu \mathrm{l} \\ \text { cDNA/DNA template } & 1 \mu \mathrm{l}\end{array}$

Table 13. General temperature profile for PCR with Q5 High Fidelity Polymerase.

\begin{tabular}{lccc}
\hline Step & Temperature $\left[{ }^{\circ} \mathbf{C}\right]$ & Time $[\mathrm{min}]$ & Repeats \\
\hline $\begin{array}{l}\text { Initial } \\
\text { denaturation }\end{array}$ & 98 & $00: 30$ & $1 \mathrm{x}$ \\
\hline Denaturation & 98 & $00: 15$ & \\
Annealing & 55 & $00: 15$ & $30 \mathrm{x}$ \\
Elongation & 72 & $00: 30 / \mathrm{kb}$ & \\
\hline $\begin{array}{l}\text { Final } \\
\text { Extension }\end{array}$ & 72 & $2: 00$ & $1 \mathrm{x}$ \\
Final Hold & 4 & $5: 00$ & $1 \mathrm{x}$ \\
\hline
\end{tabular}

\subsubsection{Agarose gel electrophoresis}

To separate and visualize DNA fragments or to determine the RNA quality, an agarose gel electrophoresis was performed. Therefore, samples were mixed with $6 \mathrm{x}$ loading dye and loaded onto a $1 \%-3 \%$ agarose gel by gel electrophoresis. To prepare the gel, the respective amount of agarose was mixed with $1 \mathrm{x}$ TAE buffer and heated in the microwave until the agarose was dissolved completely. The mix was then allowed to cool down to $\sim 50^{\circ} \mathrm{C}$ and $5 \mu \mathrm{HD}$ Green (Intas Science Imaging Instruments $\mathrm{GmbH}$, Göttingen, Germany) were added to $100 \mathrm{ml}$ agarose. The solid gel was put into Sub-Cell GT tank (BioRad, Munich, Germany) and the tank was filled with 1x TAE buffer. GeneRuler $^{\mathrm{TM}} 1 \mathrm{kB}$ or 100 bp DNA ladder (Thermo Fisher Scientific, Waltham, USA) as well as the samples were loaded into the wells. The gels ran at 90-120V for about $30 \mathrm{~min}$. After the run, the gel was analysed with a G:Box Genoplex Transilluminator gel documentation and analysis system (VWR, Lutterworth, UK). 


\subsubsection{Purification of DNA fragments}

PCR fragments used for cloning were cleaned-up directly. For PCR product purification, the GeneJET GelExtraction and DNA CleanUp Micro Kit (Thermo Fisher Scientific, Waltham, USA) was used.

\subsubsection{Gibson Assembly}

Gibson assembly to generate pPICZaA-bgh06777 and pPICZaA-bgh00220 based on the protocol by Gibson et al., 2009. A molar ratio of insert to vector from 2:1 was used.

The cloning was carried out as follows: The generation of pPICZaA-bgh06777 and pPICZaAbgh00220 required two fragments. The first fragment (1) represents the plasmid backbone and was amplified from pPICZ $\alpha$ A (Thermo Fisher Scientific, Waltham, USA). Bgh06777 without the native signal peptide (nucleotides encoding amino acid 19-293) that additionally carried a 3 ' and 5' overhang matching pPICZ $\alpha$ A or Bgh00220 without the native signal peptide (nucleotides encoding amino acid 19-293) that additionally carried a 3' and 5' overhang matching pPICZaA represented the second fragment (2). The second fragment was amplified from cDNA generated from $H$. vulgare plants infected with Blumeria graminis f.sp. hordei. The primers for amplification of the two fragments were designed using the Nebuilder web tool (New England Biolabs, Frankfurt/Main, Germany) and are listed in table Table 5. Upon incubation of the respective two fragments with the Gibson Master Mix for $1 \mathrm{~h}$ at $50^{\circ} \mathrm{C}$ and $1 \mathrm{~min}$ at $95^{\circ} \mathrm{C}, 2 \mu \mathrm{l}$ of the Gibson assembly mix were directly transformed into E. coli TOP $10 \mathrm{~F}$ ' cells.

\subsubsection{Sequencing of DNA}

Generated plasmids were sequenced by genewiz (Vancouver, Canada) or Seqlab (Götingen, Germany). Sequencing reactions were prepared according to Genewiz or Seqlab sample requirements, respectively. The results were analysed with either ApE-A plasmid editor software v2.0.53c (California, USA) or the bioinformatics software Geneious version 7.1.5.

\subsubsection{Restriction enzyme digest of DNA}

The restriction enzymes used in this study were standard enzymes of Thermo Fisher Scientific (Waltham, USA). The enzymes were used according to the manufacturer's instructions.

For restriction digestions to linearize plasmids used for $P$. pastoris transformation, $10 \mu \mathrm{l} 10 \mathrm{x}$ buffer were mixed with $50 \mathrm{U}$ Pmel and $10 \mu \mathrm{g}$ plasmid DNA. This mix was filled up with water to $100 \mu \mathrm{l}$ and incubated for $2 \mathrm{~h}$ at $37^{\circ} \mathrm{C}$ while shaking. Digestion products were analysed via agarose gel electrophoresis.

\subsubsection{Isolation of RNA from plant material}

Total RNA of $A$. thaliana seedlings and $H$. vulgare leaves infected with Blumeria graminis f.sp. hordei was isolated using the TRIZOL extraction method (Chomczynski, 1993).

Upon treatment, four $14 \mathrm{~d}$ old $A$. thaliana seedlings were transferred into $2 \mathrm{ml}$ tubes containing stainless steel balls and frozen in liquid nitrogen. The seedlings were stored at $-80^{\circ} \mathrm{C}$ until use. For RNA extraction from $H$. vulgare, leaves of $10 \mathrm{~d}$ old plants infected with Bgh were harvested and 
frozen in liquid nitrogen. The plant material was ground to a fine powder using a TissueLyser LT (Qiagen, Hilden, Germany) and stored at $-80^{\circ} \mathrm{C}$ until use.

$1 \mathrm{ml}$ Qiazol (company) were added to the powder and incubated for $10 \mathrm{~min}$ at RT on a shaker. Thereafter, $200 \mu \mathrm{l}$ chloroform were added and again the samples were incubated for $10 \mathrm{~min}$ at RT on a shaker. The samples were then centrifuged for $60 \mathrm{~min}$ at $4^{\circ} \mathrm{C}$ at $13.000 \mathrm{rpm}$. $600 \mu \mathrm{l}$ of the supernatant were transferred to a new tube and mixed with $440 \mu \mathrm{l}$ isopropanol. The tubes were inverted several times and incubated for $10 \mathrm{~min}$ at $4^{\circ} \mathrm{C}$. For precipitation of RNA, the samples were centrifuged for $15 \mathrm{~min}$ at $4^{\circ} \mathrm{C}$ at 13.000 . After centrifugation, the supernatant was discarded and the RNA pellet was washed with $500 \mu \mathrm{l} 70 \% \mathrm{EtOH}$. The supernatant was discarded and the RNA pellet was air-dried for $15 \mathrm{~min}$ and finally resuspended in $50 \mu \mathrm{l}$ RNase-free water. To solve the RNA, the tubes were incubated for $10 \mathrm{~min}$ at $65^{\circ} \mathrm{C}$ in a thermomixer. The RNA concentration was measured using NanoDrop and used for cDNA synthesis.

\subsubsection{DNase I digestion of RNA}

Before the RNA was used for cDNA synthesis, RNA samples were digested with DNase I (Thermo Fisher Scientific; Waltham, MA, USA) according to the manufacturer's instructions.

$17 \mu \mathrm{l}$ RNA were mixed with $1 \mu \mathrm{L} 10 \times$ DNase I buffer with $\mathrm{MgCl}_{2}, 1 \mu \mathrm{l}$ DNase I $(1 \mathrm{U} / \mu \mathrm{l})$ and $0.5 \mu \mathrm{l}$ RiboLock RNase Inhibitor (Thermo Fisher Scientific; Waltham, MA, USA). This mixture was incubated at $37^{\circ} \mathrm{C}$ for $30 \mathrm{~min}$ and next, $1 \mu \mathrm{l}$ EDTA $(50 \mathrm{mM})$ was added. The samples were further incubated at $65^{\circ} \mathrm{C}$ for $10 \mathrm{~min}$ at finally at $4^{\circ} \mathrm{C}$ for $2 \mathrm{~min}$. The DNase I digested samples were then used for cDNA synthesis.

\subsubsection{1 cDNA synthesis}

DNase I digested RNA samples were used for cDNA synthesis according to the manufacturer's instructions (Thermo Fisher Scientific; Waltham, MA, USA).

First, DNase I digested RNA was adjusted to $250 \mathrm{ng} / \mu \mathrm{l}$ and $1 \mu \mathrm{g}$ RNA was used for cDNA synthesis. The adjusted RNA was combined with $2 \mu \mathrm{l}$ oligodT primer [100 $\mu \mathrm{g} / \mu \mathrm{l}]$ and incubated for $10 \mathrm{~min}$ at $70^{\circ} \mathrm{C}$. Upon cooling down to $4^{\circ} \mathrm{C}, 4 \mu \mathrm{l} 5 \times \mathrm{M}$-MulVRT buffer, $2 \mu \mathrm{l}$ dNTPs [10 mM], $1 \mu \mathrm{l}$ reverse transcriptase (RevertAid ${ }^{T M} \mathrm{H}$ Minus M-MulVRT $200 \mathrm{U} / \mu \mathrm{l}$ ) were added to the samples. The samples were then incubated for $70 \mathrm{~min}$ at $42^{\circ} \mathrm{C}$, followed by an incubation for $10 \mathrm{~min}$ at $70^{\circ} \mathrm{C}$ and finally, for 2 min at $4^{\circ} \mathrm{C}$. For quantitative RT-PCR, samples were diluted $1: 500$ and stored at $-20^{\circ} \mathrm{C}$.

\subsubsection{Quantitative reverse transcription PCR (qRT-PCR)}

For qRT-PCR, the amplification and simultaneous quantification was carried out with the CFX96 Touch TM Real-Time PCR Detection System with the CFX Manager TM Software and the respective qRT-PCR-96-well plates (BioRad, Hercules, CA, USA). Reactions were set up as described below in Table 14 with Sso Fast EvaGreen supermix (BioRad, Munich, Germany). 
Table 14. qRT-PCR reaction mix.

\begin{tabular}{cc}
\hline Component & Volume per $\mathbf{1 0} \boldsymbol{\mu l}$ reaction $[\boldsymbol{\mu l}]$ \\
\hline SsoFast EvaGreen supermix & 5 \\
Forward Primer $(2 \mu \mathrm{M})$ & 1 \\
Reverse Primer $(2 \mu \mathrm{M})$ & 1 \\
cDNA & 3 \\
\hline
\end{tabular}

The reactions were pipetted into clear 96 well plates (BioRad, Munich, Germany) with three technical replicates per sample. The following protocol was used (Table 15) for amplification and simultaneous quantifications.

Table 15. qRT-PCR program.

\begin{tabular}{cccc}
\hline Step & Temperature & Time & Repeats \\
\hline Denaturation & $95^{\circ} \mathrm{C}$ & $30 \mathrm{sec}$ & \\
Annealing & $95^{\circ} \mathrm{C}$ & $5 \mathrm{sec}$ & $45 \mathrm{x}$ \\
Extension & $55^{\circ} \mathrm{C}$ & $10 \mathrm{sec}$ & \\
\hline Melting curve & $60-95^{\circ} \mathrm{C}$ & $5 \mathrm{sec}$ & - \\
\hline
\end{tabular}

\subsubsection{Calcium Assays}

For analysing the influx of calcium ions upon MAMP or carbohydrate treatment, aequorin luminescence measurements were performed using Col-0 seedlings expressing the calcium-sensing protein aequorin. The assay was performed as described in Ranf et al., 2012 with little changes. The measurements were carried out using 96-wellplates and a TECAN infinite ${ }^{\circledR}$ M200 plate reader (Tecan Group Ltd, Männedorf, Switzerland).

Each well was filled with $75 \mu \mathrm{lddH_{2 }}$ O. 7-9d old seedlings grown in-vitro were transferred one by one into the wells of a 96-well plate. One seedling was transferred into one well and should be covered fully with water. The coelenterazine working solution was prepared and $25 \mu \mathrm{l}$ of the working solution were directly transferred into each well, resulting in an end concentration of $10 \mu \mathrm{M}$ coelenterazine. The plate was covered and incubated overnight in the dark at room temperature.

The next day, MAMP and carbohydrate working solutions were prepared. Therefore, the respective MAMPs or carbohydrates were diluted in a 3-fold concentration in $\mathrm{dd}_{2} \mathrm{O}$. Usually the MAMPs and carbohydrates were used at the following end concentrations: chitin $[100 \mu \mathrm{g} / \mathrm{ml}]$, flg22 [50 nM] and carbohydrates [10, 50 or $100 \mu \mathrm{g} / \mathrm{ml}]$. The carbohydrates used are listed in Table 7 .

Next, the resting levels were measured. Therefore, the first wells were scanned every $6 \mathrm{sec}$ for $1 \mathrm{~min}$ with $150 \mathrm{~ms}$ integration time. In the next step, $50 \mu \mathrm{l}$ of the 3-fold concentrated MAMP or carbohydrate solutions were added to the first row. The luminescence $(L)$ upon treatment is recorded in $6 \mathrm{sec}$ 
intervals for $20 \mathrm{~min}$ with $150 \mathrm{~ms}$ integration time. Finally, to measure the total remaining luminescence wells were discharged by adding $150 \mu \mathrm{l}$ discharge solution and subsequent scanning of the wells for $3 \mathrm{~min}$ in a $6 \mathrm{sec}$ interval. After completion of the measurements of the first row, the remaining rows were measured accordingly. As negative control, seedlings of one of the rows were treated with $50 \mu \mathrm{l}$ $\mathrm{ddH}_{2} \mathrm{O}$ instead of a 3 -fold concentrated MAMP or carbohydrate solution.

The $\mathrm{Ca}^{2+}$ concentrations were calculated and normalized according to Rentel and Knight, 2004 and are depicted as $L / L_{\max }$ with $L$ representing the luminescence at any time point upon carbohydrate or MAMP treatment and $L_{\max }$ representing the total remaining aequorin. To calculate $L_{\max }$, the luminescence obtained upon treatment with the discharge solution was integrated.

\subsubsection{ROS Burst Assays}

For analysing the production of reactive oxygen species (ROS) upon MAMP or carbohydrate treatment in $A$. thaliana and barley, a chemiluminescence-based assay was performed using 96-well plates. For the standard set-up of this work, several treatments were carried out on one plate.

Each well was filled with $100 \mu \mathrm{l}$ tap water. For analysing the ROS production in A. thaliana, 3-4 leaves per 5-7 week-old plant were harvested. Per leaf, 3-4 leaf discs with a diameter of $4 \mathrm{~mm}$ were harvested and transferred into the wells. Leaf discs of one plant were transferred into one row of the 96-well plate. Thus, eight plants were needed for one plate.

For analysing the ROS production in $\mathrm{H}$. vulgare, 12 leaf discs of a diameter of $4 \mathrm{~mm}$ were harvested from second leaves of 10-14 day old $H$. vulgare plants and transferred into the wells. Leaf discs of one plant were transferred into one row of the 96 well plate. The plate was wrapped into a plastic bag und incubated overnight at room temperature.

The next day, the L-012 solution was prepared. The water in the wells was removed carefully and replaced with either $100 \mu \mathrm{l}$ L-012 solution, L-012 solution with chitin $(100 \mu \mathrm{g} / \mathrm{ml})$, L-012 solution with flg22 (100 nM) or L-012 solution with carbohydrate $(100 \mu \mathrm{g} / \mathrm{ml})$ per well. The carbohydrates used are listed in table Table 7. The L-012 solution was added to the leaf discs directly before starting the measurement. The chemiluminescence was measured every minute over a period of $1 \mathrm{~h}$ using a TECAN infinite ${ }^{\circledR}$ M200 plate reader (Tecan Group Ltd, Männedorf, Switzerland). The obtained data were analysed with Excel.

\subsubsection{Hydrolysis of $\beta-1,3 ; 1,4-p o l y m e r$}

To generate MLG oligosaccharide of varying length that were tested for their ability to act as elicitor, the respective $\beta-1,3 ; 1,4$-polymer was hydrolysed. Therefore, $10 \mathrm{mg} / \mathrm{ml}$ barley $\beta-1,3 ; 1,4$-polymer (Megazyme, Ireland) was dissolved in $100 \mathrm{mM}$ Sodium Phosphate buffer $(\mathrm{pH}=6.5)$ by heating and stirring. The solution was allowed to cool down and then either $0.025 \mathrm{U} \mathrm{ml}^{-1}$ or $0.05 \mathrm{U} \mathrm{ml}^{-1}$ B. subtilis lichenase (Megazyme, Ireland) were added to the solution. The hydrolysate was incubated for 0, 5, $15,30,45,60,120$ and $240 \mathrm{~min}$ at $40^{\circ} \mathrm{C}$ at $160 \mathrm{rpm}$. To inactivate the enzyme, the solution was incubated for $15 \mathrm{~min}$ in boiling water. The hydrolysates were stored at $\mathrm{RT}$ or at $4^{\circ} \mathrm{C}$.

To generate MLG oligosaccharides of varying lengths for the forward and reverse genetic screen, $10 \mathrm{mg} / \mathrm{ml}$ barley $\beta-1,3 ; 1,4$-polymer (Megazyme, Ireland) was dissolved in $100 \mathrm{mM}$ Sodium 
Phosphate buffer $(\mathrm{pH}=6.5)$ by heating and stirring. The solution was allowed to cool down and $1 \mathrm{U} \mathrm{ml}^{-1} \mathrm{~B}$. subtilis lichenase was added to the solution. The solution was incubated at $40^{\circ} \mathrm{C}$ at 160 rpm for $1 \mathrm{~h}$ and the reaction was inactivated by incubating the hydrolysate in boiling water for $15 \mathrm{~min}$. The hydrolysate was stored at RT or at $4^{\circ} \mathrm{C}$.

\subsubsection{Biochemical methods}

\subsubsection{Protein extraction of $\boldsymbol{A}$. thaliana}

Protein extracts were prepared from 14-day old $A$. thaliana seedlings grown in an in-vitro culture. Upon treatment, 4 seedlings were transferred into one $1.5 \mathrm{ml}$ tube and frozen in liquid nitrogen and stored at $-80^{\circ} \mathrm{C}$ until usage. The frozen seedlings were ground with $200 \mu \mathrm{l}$ CERK1 extraction buffer and half of a spatula of quartz sand using the IKA ${ }^{\circledR}$ RW digital drill (IKA-Werke, Staufen, Germany). The pistil was cleaned with another $200 \mu$ of CERK1 extraction buffer and the sample was filled up with CERK1 extraction buffer to a volume of $600 \mu$ l. The samples were centrifuged for $10 \mathrm{~min}$ at $13.000 \mathrm{rpm}$ at $4^{\circ} \mathrm{C}$. The supernatant was transferred into a new tube and the protein concentration was determined via Bradford Assay (see 2.2.5.3). The concentrations were equalized to $1-1.5 \mu \mathrm{g} / \mathrm{ml}$. $25 \mu \mathrm{l}$ of the adjusted protein extract were then mixed with $75 \mu \mathrm{l} 4 \mathrm{x}$ SDS buffer and stored at $-20^{\circ} \mathrm{C}$ until usage.

\subsubsection{Protein extraction of $\boldsymbol{H}$. vulgare}

Protein extracts were prepared from $H$. vulgare leaf discs that were treated with different MAMPs and mixed linkage glucans. Upon treatment, the leaf discs were carefully transferred into a $1.5 \mathrm{ml}$ tube and frozen in liquid nitrogen and stored at $-80^{\circ} \mathrm{C}$ until use.

The frozen leaf discs were ground with $200 \mu \mathrm{l}$ barley extraction buffer and half of a spatula of quartz sand using the IKA ${ }^{\circledast}$ RW digital drill (IKA-Werke, Staufen, Germany). Upon grinding, the pistil was rinsed with another $200 \mu \mathrm{l}$ barley extraction buffer and the sample was filled up to a volume of $600 \mu \mathrm{l}$ with barley extraction buffer. The samples were centrifuged for $10 \mathrm{~min}$ at $13.000 \mathrm{rpm}$ at $4^{\circ} \mathrm{C}$. The supernatant was transferred into a new tube. The protein concentration was calculated using the Bradford assay (see 2.2.5.3). The concentrations were equalized and $25 \mu \mathrm{l}$ of the samples were mixed with $4 \times$ SDS buffer and stored at $-20^{\circ} \mathrm{C}$ until use.

\subsubsection{Protein quantification via Bradford Assay}

The protein concentration of the prepared protein extracts were quantified according to Bradford (Bradford, 1976). The Bradford reagent (Roti-Quant, Roth, Karlsruhe, Germany) was diluted 1:5 in $\mathrm{ddH}_{2} \mathrm{O}$. Next, $2 \mu \mathrm{l}$ of each sample were mixed with $1 \mathrm{ml}$ Bradford reagent. Additionally, a dilution series of $0,3,5,7,10$ and $15 \mu \mathrm{g} / \mathrm{ml}$ bovine serum albumin was prepared. The samples were incubated for 10 min at RT and the absorbance at $595 \mathrm{~nm}$ was measured with a WPA Biowave II photometer (Biochrom AG, Berlin, Germany). A standard curve was generated by plotting $A_{595}$ against the respective concentration. The generated standard curve was then used to calculate the protein concentration of the samples. To equalize protein concentrations, the samples were adjusted to $1-$ $1.5 \mu \mathrm{g} / \mathrm{ml}$ with CERK1 or barley extraction buffer. 


\subsubsection{SDS-polyacrylamide gel electrophoresis (SDS-PAGE)}

To separate protein according to their molecular mass, a SDS-PAGE was performed.

First, the resolving gel was mixed (see Table 16) and poured between two glass plates with a spacing of $1.5 \mathrm{~mm}$ in the respective gel stand and overlaid with isopropanol. Upon polymerization at RT, the isopropanol was removed and the stacking gel (see Table 16) was poured onto the resolving gel. Directly after pouring, a comb was inserted. The concentration of the resolving gel depends on the expected protein size and the purpose of the experiment. For immunoblot analysis, only $10 \%$ SDS gels with $1.5 \mathrm{~mm}$ spacing were used. For expression studies, Mini Protean TGX (4-20\%) 15/10 well gels (BioRad, Munich, Germany) were used.

SDS-PAGE was performed in the Mini-PROTEAN ${ }^{\circledR} 3$ System (BioRad, Munich, Germany). The gels were placed into the gel apparatus and the tank was filled up with 1x SDS running buffer. Before loading the samples to the gel, they were incubated for $3-5 \mathrm{~min}$ at $95^{\circ} \mathrm{C}$ and up to $20 \mu \mathrm{l}$ were loaded depending on the comb size. As a size marker, PageRuler ${ }^{\mathrm{TM}}$ Prestained Plus protein Ladder (Thermo Fisher Scientific, Waltham, USA) was used. For immunoblot analysis, $1.5 \mathrm{~mm}$ gels ran at $30 \mathrm{~mA}$ per gel until the bromphenolblue front reached the end of the gel. For expression studies, the gel ran at $150 \mathrm{~V}$ until the bromphenolblue front reached the end of the gel. The apparatus was then disassembled and the gels were either used for immunoblot analysis or directly stained with Coomassie blue.

Table 16. Composition of SDS PAGE Gel buffers and mixes used in this study.

SDS PAGE Gel Buffer (250 ml)

\begin{tabular}{|c|c|c|}
\hline $10 \%$ resolving gel buffer & $\begin{array}{l}1 \mathrm{M} \text { Tris }-\mathrm{HCl}(\mathrm{pH}=8.8) \\
\mathrm{SDS}(10 \%) \\
\mathrm{ddH}_{2} \mathrm{O}\end{array}$ & $\begin{array}{l}143.6 \mathrm{ml} \\
3.79 \mathrm{ml} \\
102.53 \mathrm{ml}\end{array}$ \\
\hline Stacking gel buffer & $\begin{array}{l}1 \mathrm{M} \text { Tris }-\mathrm{HCl}(\mathrm{pH}=6.8) \\
\mathrm{SDS}(10 \%) \\
\mathrm{ddH}_{2} \mathrm{O}\end{array}$ & $\begin{array}{l}38.85 \mathrm{ml} \\
3.06 \mathrm{ml} \\
208.24 \mathrm{ml} \\
\end{array}$ \\
\hline \multicolumn{3}{|c|}{ SDS-PAGE gel mixes (10 ml) } \\
\hline $10 \%$ resolving gel & $\begin{array}{l}10 \% \text { resolving gel buffer } \\
30 \% \text { acrylamide } \\
\text { APS }(10 \%) \\
\text { TEMED }\end{array}$ & $\begin{array}{l}6.6 \mathrm{ml} \\
3.3 \mathrm{ml} \\
0.1 \mathrm{ml} \\
0.004 \mathrm{ml}\end{array}$ \\
\hline Stacking gel & $\begin{array}{l}\text { Stacking gel buffer } \\
30 \% \text { acrylamide } \\
\text { APS (10\%) } \\
\text { TEMED }\end{array}$ & $\begin{array}{l}8.16 \mathrm{ml} \\
1.66 \mathrm{ml} \\
0.05 \mathrm{ml} \\
0.005 \mathrm{ml}\end{array}$ \\
\hline
\end{tabular}




\title{
2.2.5.5 Immunoblot analysis (Western Blot)
}

Extracted proteins were separated via SDS-PAGE before immunoblot analysis. Proteins were transferred via electroblotting using the TRANS-BLOT ${ }^{\circledR}$ CELL (BioRad, Munich, Germany) onto a PVDF membrane with a pore size of $0.45 \mu \mathrm{m}$ (Roth, Karlsruhe, Germany). To this end, the PVDF membrane was activated by briefly dipping it into methanol before applying it to the gel. The blotting apparatus was assembled as follows:

\author{
cathode \\ black grid of the clamp \\ sponge \\ Whatman paper \\ gel (facing the cathode) \\ PVDF membrane \\ Whatman paper \\ sponge \\ transparent/red grid of the clamp \\ anode
}

The blotting was performed in $1 \mathrm{x}$ transfer buffer at $4^{\circ} \mathrm{C}$ for $2 \mathrm{~h}$ at $80 \mathrm{~V}$.

Upon blotting, the PVDF membrane was blocked for at least $1 \mathrm{~h}$ with $10 \mathrm{ml}$ TBST + 4\% MP at RT on a rotary shaker. After blocking, the PVDF membrane was incubated with the primary antibody overnight at $4^{\circ} \mathrm{C}$ on a rotary shaker. Upon incubation with the primary antibody, the primary antibody was removed and the PVDF membrane was washed 5 times with TBS-T + 4\% MP for 12 min. Next, the PVDF membrane was incubated for $2 \mathrm{~h}$ with the secondary antibody at RT on a rotary shaker. The used antibodies are listed in Table 10. Upon incubation with the second antibody, the PVDF membrane was washed 5 times with TBS-T for 12 min. Next, the PVDF membrane was equilibrated for $5 \mathrm{~min}$ in AP buffer. $500 \mu \mathrm{L}$ Immun-Star TM AP substrate (BioRad, Munich, Germany) was added to each membrane. The membranes were wrapped in plastic foil and incubated for 5-10 min in the dark. Upon incubated, the membranes were transferred to a new plastic bag and the chemiluminescence was detected using a detection device (ChemiDoc Touch; BioRad, Munich, Germany).

\subsubsection{Coomassie Staining of PVDF membranes and SDS gels}

\subsection{PVDF membranes}

To visualize protein bands, PVDF membranes were stained with Coomassie brilliant blue. To this end, PVDF membranes were covered with Coomassie staining solution and incubated for 5 min while shaking at RT. After incubation, the PVDF membrane was rinsed in water and the background was 
then removed by incubating the membrane in destaining solution at RT while shaking. Finally, the membrane was rinsed in water again and dried.

\subsection{SDS gels}

To visualize protein bands on SDS gel, the gel was stained with Coomassie Blue. Therefore, the gel was incubated in Coomassie Staining solution for $5 \mathrm{~min}$ at room temperature while shaking. To remove background staining, the Coomassie staining solution was removed and the gel was covered with water and incubated in the microwave for $1 \mathrm{~min}$. Then, the gel was incubated in water at room temperature while shaking. Depending on the staining intensity, the water had to be removed and the gel had to be put into the microwave several times.

\subsubsection{Thin Layer Chromatography (TLC)}

First, a TLC chamber was filled with TLC running buffer. In a next step, $5 \mu \mathrm{l}$ of the analytes were dropped onto the TLC Silica gel plate (Merck, Darmstadt, Germany) and were allowed to dry at RT. Upon drying, the TLC plate was put into the chamber and the running buffer was allowed to run up the plate until it almost reached the top. The plate was again allowed to dry at room temperature under the fume hood. To stain the TLC, the TLC plate was wetted with TLC staining solution and then incubated on a heating plate at $99^{\circ} \mathrm{C}$ for $30-45 \mathrm{~min}$.

\subsubsection{Expression of His-tagged protein in $P$. pastoris}

\subsection{Small Scale Expression of secreted proteins in P. pastoris}

To test expression of the gene of interest in the obtained $P$. pastoris transformants, a small scale expression was performed. Therefore, the transformants were grown under four different conditions for 7 days: The transformants were either grown at $16^{\circ} \mathrm{C}$ or at $25^{\circ} \mathrm{C}$ and fed with either $1 \%$ or $3 \%$ methanol (end concentration in the medium).

In the morning, yeast transformants were inoculated in a $50 \mathrm{ml}$ tube containing $3 \mathrm{ml}$ YPD and either 100 or $500 \mu \mathrm{g} / \mathrm{ml}$ Zeocin. The cultures were incubated over the day at $30^{\circ} \mathrm{C}$ at $225 \mathrm{rpm}$. In the afternoon, $600 \mu \mathrm{L}$ of the pre-culture were inoculated in $10 \mathrm{ml} \mathrm{BMGY}$ in a $50 \mathrm{ml}$ tube and incubated overnight at $30^{\circ} \mathrm{C}$ at $225 \mathrm{rpm}$. The cells were incubated in BMGY to ensure an optimal induction of expression in presence of methanol. Per transformant, four new tubes were inoculated. In the afternoon of the next day, all grown cultures were centrifuged for $15 \mathrm{~min}$ at $4.000 \mathrm{~g}$ at RT. The supernatant was discarded and the pellets were resuspended in $4 \mathrm{ml}$ BMMY (containing $3 \%$ methanol). The cultures were further grown at either $16^{\circ} \mathrm{C}$ or $25^{\circ} \mathrm{C}$ at $160 \mathrm{rpm}$. The transfer of the cultured from BMGY to BMMY medium represents day 1 of the small scale expression. From the third day on, the cultures were fed every day with either $1 \%$ methanol ( $50 \mu \mathrm{l}$ of $100 \%$ methanol) or $3 \%$ (150 $\mu \mathrm{l}$ of $100 \%$ methanol) methanol. Furthermore, samples were taken for testing the expression at day 3 and day 5 . Therefore, $90 \mu \mathrm{l}$ of the cultures were transferred into a $1.5 \mathrm{ml}$ tube, centrifuged for $3 \mathrm{~min}$ at $10.000 \mathrm{~g}$ and frozen in liquid nitrogen. The samples were stored at $-20^{\circ} \mathrm{C}$ until use. On day 7 , the yeast cultures were centrifuged for $15 \mathrm{~min}$ at $3000 \mathrm{~g}$ at $4^{\circ} \mathrm{C}$. The supernatant was filtered with a filter having a pore size of $0.22 \mu \mathrm{m}$ into a $15 \mathrm{ml}$ tube. The supernatant can be stored at $4^{\circ} \mathrm{C}$ until use. 
To test for the presence of the protein in any of the supernatants, $40 \mu \mathrm{l}$ of the supernatant obtained at day 7 were mixed with $10 \mu \mathrm{l}$ SDS loading dye and used for SDS-PAGE.

According to the results of the small scale expression, a $P$. pastoris transformant was chosen that had the highest protein abundance at a particular growth condition for large scale expression.

\subsection{Large Scale Expression of secreted proteins in P. pastoris}

For expression of Bgh06777, a transformant was chosen that had the highest abundance of the protein while growing at $16^{\circ} \mathrm{C}$ with $1 \%$ methanol. For purification of the protein, the supernatant was collected at day 5 of the large scale expression.

In the morning, the respective yeast transformant was inoculated four times in $5 \mathrm{ml}$ YPD containing $500 \mu \mathrm{g} / \mathrm{ml}$ Zeocin in $50 \mathrm{ml}$ tubes. The cultures were grown over the day at $30^{\circ} \mathrm{C}$ at $160 \mathrm{rpm}$. In the afternoon, $10 \mathrm{ml}$ of the pre-cultures were used to inoculate $500 \mathrm{ml} \mathrm{BMGY}$ in a 2l baffled flask. The cultures were grown overnight at $30^{\circ} \mathrm{C}$ at $220 \mathrm{rpm}$ to an $\mathrm{OD}_{600}$ of $5-6$. The next day, the cultures were centrifuged at $3700 \mathrm{rpm}$ at RT. The supernatant was discarded and the cells resuspended in $400 \mathrm{ml}$ BMMY (containing 3\% methanol). The cultures were transferred to a 21 baffled flask and incubated at $16^{\circ} \mathrm{C}$ at $250 \mathrm{rpm}$ (day 1 ). The culture was fed every $24 \mathrm{~h}$ beginning of day 3 with $100 \%$ methanol to maintain a concentration of $1 \%$ methanol in the medium. On day 5 , the culture was centrifuged for $10 \mathrm{~min}$ at $3700 \mathrm{rpm}$ at RT and the supernatant was transferred to a beaker. After filtration of the supernatant, the $\mathrm{pH}$ of the supernatant was adjusted to 7.7 with $\mathrm{NaOH}$ and stored at $4^{\circ} \mathrm{C}$ until use for extraction and purification of the protein.

\subsubsection{Extraction and purification of His-tagged protein in $P$. pastoris}

To extract and purify BGH06777 from $P$. pastoris, the supernatant obtained from the large scale expression was first reduced in volume using Vivaflow 200 (Sartorius, Göttingen, Germany). The supernatant was then loaded onto a $1 \mathrm{ml}$ HisTrap IMAC FF nickel-nitrilotriacetic acid column (GE Healthcare, Chicago, USA) using the Minipulse 3 Peristaltic Pump (Gilson, France). After loading of the supernatant, the column was washed with $5 \mathrm{ml}$ buffer $\mathrm{A}$. Next, the protein was eluted from the column with the FPLC Biologic Duoflow system (BioRad, Munich, Germany) using a linear gradient of $0-100 \%$ buffer $\mathrm{C}$. The fractions were monitored with $\mathrm{A}_{280}$ and collected with the Fraction Collector (Biofraction, BioRad, Munich, Germany). The fractions with the eluted protein were pooled and exchanged with SEC buffer using a 3 kDa Vivaspin centrifugal filter (GE Healthcare, Chicago, USA). The protein concentration was determined with the Epoch Microplate Spectrophometer (BioTek, Winooski, USA) and the molar extinction coefficient (BGH06777 $\left.=2.3 \mathrm{M}^{-1} \mathrm{~cm}^{-1}\right)$. The molar extinction coefficient was calculated using the ProtParam tool of the ExPASy Bioinformatics Resource Portal (Gasteiger et al., 2015). Upon concentrating the protein to $1 \mathrm{mg} / \mathrm{ml}$, aliquots were frozen in liquid nitrogen and stored at $-80^{\circ} \mathrm{C}$. The protein purity was determined by SDS-PAGE. 


\subsubsection{Functional characterization of enzymes}

\subsection{Product Analysis using HPAEC-PAD}

Carbohydrate Analysis was carried out using High-performance-anion-exchange chromatography with pulsed amperometric detection (HPAEC-PAD). HPAEC-PAD was performed using a Dionex ICS-5000 HPLC system equipped with an AS-AP autosampler in a sequential infection configuration using the Chromelion software version 7. $10 \mu \mathrm{l}$ of the samples were injected on a $3 \times 250 \mathrm{~mm}$ Dionex Carbopac PA200 column (Thermo Scientific, Waltham, USA). This equipment was used for all separations. The gradient was used as follows: $0-5 \mathrm{~min}, 10 \% \mathrm{~B}, 0 \% \mathrm{C}$ (initial conditions); 5-12 min 10\% B, linear gradient from $0-30 \%$ C; $12.0-12.1 \mathrm{~min}, 50 \% \mathrm{~B}, 50 \% \mathrm{C} ; 12.1-13.0 \mathrm{~min}$, exponential gradient of $B$ and $\mathrm{C}$, back to initial conditions, 13-17 min initial conditions. Solvent $A$ was ultrapure water, solvent $B$ was $1 \mathrm{M}$ sodium hydroxide and solvent $\mathrm{C}$ was $1 \mathrm{M}$ sodium acetate.

\subsection{Confirmation of protein mass by mass-spectrometry}

The intact protein mass was determined using a Waters Q-Tof with a nanoACQUITY UPLC system according to Sundqvist et al., 2007

\subsection{Identification of substrates}

To identify the substrate of BGH06777, $1 \mu \mathrm{g} / \mathrm{ml}$ BGH06777 in $50 \mathrm{mM}$ MES buffer $(\mathrm{pH}=5)$ was incubated with either $0.05 \mathrm{mM}$ laminarihexaose, $0.05 \mathrm{mM}$ laminaripentaose, $0.05 \mathrm{mM}$ laminaritetraose or $0.05 \mathrm{mM}$ laminaritriose. Upon $1 \mathrm{~h}$ (partial digest) and $12 \mathrm{~h}$ (full digest) incubation at $\mathrm{RT}$, the samples were subjected to product analysis using HPAEC-PAD. As standards, glucose, laminaribiose, laminaritriose, laminaritetraose, laminaripentaose and laminarihexaose were included.

\subsection{Identification of the temperature optimum}

The temperature optimum was identified in $50 \mathrm{mM}$ citrate buffer $(\mathrm{pH}=5)$ using $1 \mathrm{mM}$ laminarihexaose and $2 \mu \mathrm{g} / \mathrm{ml} \mathrm{BGH06777}$. The reaction was prepared at $4^{\circ} \mathrm{C}$ in a total volume of $1000 \mu \mathrm{l}$. The reaction was mixed well and $50 \mu \mathrm{l}$ of the digest were transferred into different PCR tubes. The $50 \mu \mathrm{l}$ digests were incubated for $1 \mathrm{~h}$ at the following temperatures [ $\left.{ }^{\circ} \mathrm{C}\right]: 30,32,35.2,39.3,44.9,49,51.9,54,55$, $57.1,60.2,64,69.3,73.5,76.3$ and 78 . Afterwards, the reactions were stopped by incubating the digests for $5 \mathrm{~min}$ at $95^{\circ} \mathrm{C}$ and $1 \mathrm{~min}$ at $4^{\circ} \mathrm{C}$. The different reactions were then transferred to HPAEC

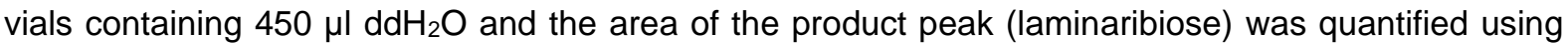
HPAEC-PAD. As standard, different concentrations of laminaribiose ranging from $0.00000156 \mathrm{mM}$ to $1 \mathrm{mM}$ were run on the HPAEC and the change in the peak area of laminaribiose was calculated using the excel linest function. The enzymatic rate was then calculated with the product peak obtained from the hydrolysis of laminarihexaose by BGH06777, the molar concentration of BGH06777 and the change of the peak area of laminaribiose (standard).

\subsection{Identification of the $\mathrm{pH}$ optimum}

To identify the $\mathrm{pH}$ optimum of BGH06777, $1 \mu \mathrm{g} / \mathrm{ml}$ BGH06777 was incubated with $1 \mathrm{mM}$ laminarihexaose in $50 \mathrm{mM}$ buffer in a total volume of $50 \mu \mathrm{l}$. The reaction was incubated in 
different buffers with different $\mathrm{pH}$ values that are listed in Table 17. The digests were incubated for $1 \mathrm{~h}$ at $25^{\circ} \mathrm{C}$ and the reactions were terminated by incubating them for $5 \mathrm{~min}$ at $95^{\circ} \mathrm{C}$. The $50 \mu \mathrm{l}$ reactions

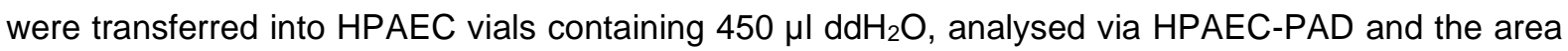
of the product peak (laminaribiose) was quantified. Different concentrations of laminaribiose (ranging from $0.00000156 \mathrm{mM}$ to $1 \mathrm{mM}$ ) were analysed via HPAEC-PAD as a standard and the change in the product peak was calculated using the linest function of Excel. Next, the enzymatic rate of BGH06777 was calculated using the molar concentration of BGH06777, the change of the peak area of laminaribiose (standard) and the laminaribiose peak areas obtained upon laminarihexaose hydrolysis with BGH067777.

Table 17. Buffers used to identify the pH optimum of BGH06777.

\begin{tabular}{ll}
\hline Buffer (Substance) & $\mathbf{p H}$ \\
\hline Citrate & 3 \\
\hline Citrate & 3.5 \\
\hline Citrate & 4 \\
\hline Citrate & 4.5 \\
\hline Citrate & 5 \\
\hline Citrate & 5.5 \\
\hline Citrate & 6 \\
\hline Citrate & 6.5 \\
\hline Citrate & 7 \\
\hline Glycin-Glycin & 8 \\
\hline Glycin-Glycin & 9 \\
\hline Glycin & 10 \\
\hline
\end{tabular}

\subsection{Michaelis-Menten Kinetics}

Michaelis-Menten kinetics were determined using HPAEC-PAD. Therefore, an enzyme stock ( 5 or 50 $\mu \mathrm{g} / \mathrm{ml} \mathrm{BGH06777}$ in $200 \mathrm{mM}$ citrate buffer $\mathrm{pH}=5.5)$ was mixed with a substrate stock ( 0.0078 to 1 $\mathrm{mM}$ final substrate concentration) preheated to $37^{\circ} \mathrm{C}$. As substrates, laminarihexaose and laminaripentaose were used. For example, $50 \mu \mathrm{l}$ of $5 \mu \mathrm{g} / \mathrm{ml}$ BGH06777 in $200 \mathrm{mM}$ citrate buffer $(\mathrm{pH}=$ 5.5) was added to $450 \mu \mathrm{l}$ of $1 \mathrm{mM}$ laminarihexaose in $\mathrm{ddH}_{2} \mathrm{O}$ preheated to $37^{\circ} \mathrm{C}$. The sample (each 10 $\mu$ ) was injected 5 times every 20 min onto the column. The change in the peak area of the resulting product laminaribiose was quantified with the linest function of excel for all used concentrations of the respective substrates. As standard, the change in the peak area of different concentrations (0.00000156 to $1 \mathrm{mM}$ ) of laminaribiose was calculated using the linest function of excel. The 
enzymatic activity was then calculated with the change of the peak area of laminaribiose generated during the hydrolysis by BGH06777, the molar concentration of BGH06777 and the change of the peak area of laminaribiose (standard). The obtained enzymatic rates were fit to the Michaelis-Menten model (Michaelis and Menten, 1913; Johnson and Goody, 2011) using OriginPro graphing software.

\subsection{Determination of the regiospecificity}

The regiospecificity of laminarihexaose hydrolysis was determined by monitoring the ${ }^{18} \mathrm{O}$ incorporation from ${ }^{18} \mathrm{O}$-water by mass spectrometry (Schagerlöf et al., 2009). $1 \mu \mathrm{L}$ BGH06777 (0.05 mg/ml bgh06777 in $1 \mathrm{M}$ citrate buffer $\mathrm{pH}=5.5$ ) and $1 \mu \mathrm{l} 10 \mathrm{mM}$ laminarihexaose were mixed with $18 \mu \mathrm{L} 97 \%$ ${ }^{18} \mathrm{O}$ water (Cambridge Isotope Laboratories) and mixed well by reciprocal pipetting. The reaction was transferred to a $50 \mu \mathrm{l}$ gas-tight Hamilton syringe 80 (Hamilton, model 1705) and infected into a Waters Xevo QTof at $2 \mu \mathrm{l} / \mathrm{min}$ using a pump (Harvard Apparatus 11 Plus). The level of isotopic labelling was quantified using the ratio: $[\mathrm{M}+\mathrm{Na}]^{+}\left({ }^{16} \mathrm{O}-1\right)$ to $[\mathrm{M}+\mathrm{Na}]^{+}\left({ }^{18} \mathrm{O}-1\right)$ (McGregor et al., 2016).

\subsubsection{Carbohydrate Analysis}

\subsection{HPAEC-PAD}

HPAEC-PAD was performed using a Dionex ICS-5000 HPLC system equipped with an AS-AP autosampler in a sequential infection configuration using the Chromelion software version 7. $10 \mu \mathrm{l}$ of the samples were injected on a $3 \times 250 \mathrm{~mm}$ Dionex Carbopac PA200 column (Thermo Scientific, Waltham, USA). $56 \mu \mathrm{M}$ of the MLG tetramer or $45 \mu \mathrm{M}$ of the MLG trimer were loaded onto the column. The following gradient was used: The gradient was used as follows: 0-5 min, 10\% B, $3.5 \% \mathrm{C}$ (initial conditions); 5-12 min 10\% B, linear gradient from $0-30 \% \mathrm{C} ; 12.0-12.1 \mathrm{~min}, 50 \% \mathrm{~B}, 50 \% \mathrm{C}$; 12.1 - $13.0 \mathrm{~min}$, exponential gradient of $B$ and $C$, back to initial conditions, 13-17 min initial conditions. Solvent A was ultrapure water, solvent $B$ was $1 \mathrm{M}$ sodium hydroxide and solvent $\mathrm{C}$ was $1 \mathrm{M}$ sodium acetate.

\subsection{MALDI-TOF}

MALDI-TOF analysis of mixed-linkage glucans was performed with a Bruker Autoflex system (Bruker Daltonics) operated in reflectron mode. $10 \mathrm{mg} / \mathrm{ml}$ of the oligosaccharide were mixed 1:5 with a 2,5-dihiydroxybenzoic acid in 1:1 $\mathrm{H}_{2} \mathrm{O}: \mathrm{MeOH}$ on a Bruker MTP 384 grounded steel MALDI plate. The samples were allowed to dry and subsequently, analysed. 


\section{Results}

\subsection{Identification and functional characterization of $B g h$ CWDEs}

\subsubsection{Identification of potential candidate GHs of Bgh}

The plant cell wall represents an effective structural barrier to microbial invasion. To breach the plant cell wall and to gain access to plant's nutrient resources, fungi secrete CWDEs that are often required for full pathogenicity (Kubicek et al., 2014). One aim of this study was to identify and functionally characterize CWDEs of the powdery mildew Bgh that are required for pathogenicity. To this end, genomic and transcriptomic data were analysed for in planta expressed and potentially secreted GHs. A genome analysis of $B g h$ conducted in 2010 revealed a reduced number of CWDEs in the genome of Bgh compared to other plant pathogenic fungi (Spanu et al., 2010). In total only two lignocellulose-degrading enzymes (Auxiliary Activity Family 9, formerly known as GH61), four hemicellulose-degrading enzymes (GH16) and one pectin/ $\beta$-1,3-glucan hydrolyzing enzyme (GH81) were identified in this study. Auxiliary activity proteins are lytic polysaccharide monooxygenases that act on cellulose or have other substrate specificities (Carbohydrate Active Enzymes database, http://www.cazy.org/, Lombard et al., 2014). However, an independent genomic analysis by our collaborators at the IPK Gatersleben (research group of the late Dr. Patrick Schweizer) identified a total of 75 CWDEs including 62 GHs belonging to 18 different GH families, four CBMs of three different families and nine CEs designated to five different families in the genome of $\mathrm{Bgh}$. Moreover, 26 putative CWDEs were identified that could not be assigned to a family (unpublished data; Dr. Patrick Schweizer, personal communication). To verify the $62 \mathrm{GHs}$ identified by our collaborators, protein sequences of all identified GHs were searched for carbohydrate active enzyme domain signatures using the publicly available sources dbCAN (http://bcb.unl.edu/dbCAN2, Yin et al., 2012) and Motif Scan (https://myhits.isb-sib.ch/cgi-bin/motif_scan). Of the 62 annotated GHs, only the protein BGH05207 designated to GH family 17 and the protein BGH02531 belonging to GH family 18 could not be verified and thus, were excluded from further analysis (Table S1).

In a next step, in planta gene expression of the $18 \mathrm{GH}$ families of $\mathrm{Bgh}$ was analysed. The expression of a gene can be evaluated by calculating the reads per million (RPM) of the total amount of reads obtained from an RNAseq experiment. RPMs were calculated for all members of the $18 \mathrm{GH}$ families identified in Bgh at different time points upon infection using publicly available in planta RNAseq data (Hacquard et al., 2013). In particular, members of the GH families 5, 16, 17, 47 and 76 were highly expressed during the compatible interaction of $\mathrm{Bgh}$ and the immunocompromised Arabidopsis triple mutant pen2 pad4 sag101 expressing MLA1 either at a specific time point of infection or throughout the whole infection indicating a potential role in pathogenicity ( 
Table S2). This indicates that they might play a role in pathogenicity and thus represent interesting candidate $\mathrm{GH}$ families for further analysis.

\subsubsection{The family GH17 was chosen for further analysis}

The different $\mathrm{GH}$ families exhibit different substrate specificities (Carbohydrate Active Enzymes database, http://www.cazy.org/, Lombard et al., 2014). To reduce the number of potential candidate families, GH families 5, 16, 17, 47 and 76 were analysed regarding their substrate specificities. Proteins designated to $\mathrm{GH}$ family 47 or 76 are known to hydrolyse mannans (Herscovics, 2001; Cuskin et al., 2015), while proteins belonging to GH5 have been shown to act e.g. on cellulose, mannans, $\beta$-1,3-glucans, $\beta$-1,6-glucans and hemicelluloses (Aspeborg et al., 2012). The GH family 16 is known to include enzymes that have activity on e.g. $\beta-1,3 ; 1,4$-glucans, hemicelluloses, $\beta-1,3$-glucans as well as chitin (Viborg et al., 2019) while family GH17 includes enzymes which mainly hydrolyse $\beta$-1,3-glucans and fungal $\beta-1,3 ; 1,6$-glucans with a region of unbranched $\beta$-1,3-linkages (Hrmova and Fincher, 1993; Carbohydrate Active Enzymes database, http://www.cazy.org/, Lombard et al., 2014). $\beta-1,3$-glucans are present in plants as callose, which is a major component of defence-associated papillae (Jacobs et al., 2003; Underwood, 2012). In order to infect a plant, pathogens do not only need to breach the cell wall but also papillae. It was shown that papillae that only contain a low amount of callose were not effective in preventing fungal penetration and haustoria formation (Chowdhury et al., 2014). This suggests that Bgh may overcome papillae with a combination of pressure and CWDEs. Since proteins designated to family $\mathrm{GH} 17$ hydrolyse $\beta-1,3$-linkages mainly in $\beta-1,3$-glucans and thus might be involved in penetration of papillae, this family was chosen for further bioinformatic analysis.

\subsubsection{Bioinformatic analysis of Bgh $\mathrm{GH} 17$ proteins}

To interact with the plant cell wall, fungal CWDEs have to be secreted and therefore, contain either a canonical signal peptide or an unconventional secretion signal. Thus, the seven members of $B g h \mathrm{GH}$ family 17 were analysed using the publicly available server SignalP (http://www.cbs.dtu.dk/services/SignalP/) and SecretomeP (http://www.cbs.dtu.dk/services/SecretomeP/) for identifying conventional or unconventional secretion signals, respectively. Except for BGH00736, which harbours an unconventional secretion signal, all GH17 family members contain a canonical N-terminal signal peptide indicating that all GH family 17 members in Bgh are likely to be secreted by the fungus in order to act on the plant cell wall (Table 18). Additionally, $\mathrm{N}$-glycosylation sites as well as phosphorylation sites were predicted with the public available tools NetNGlyc (http://www.cbs.dtu.dk/services/NetNGlyc/) and NetPhos 
(http://www.cbs.dtu.dk/services/NetPhos/), respectively. All proteins belonging to GH17 harbour at least one predicted $\mathrm{N}$-glycosylation site as well as several predicted phosphorylation sites (Table 18).

Table 18. Properties of Bgh GH 17 proteins.

\begin{tabular}{cccccc}
\hline $\begin{array}{c}\text { BluGen } \\
\text { Protein } \\
\text { Number }\end{array}$ & $\begin{array}{c}\text { Protein } \\
\text { Size } \\
{[\mathrm{kDa}]}\end{array}$ & $\begin{array}{c}\text { Predicted } \\
\text { Motif }\end{array}$ & $\begin{array}{c}\text { Predicted } \\
\text { N- } \\
\text { Glycosylation } \\
\text { Sites } \\
\text { (Position) }\end{array}$ & $\begin{array}{c}\text { Predicted } \\
\text { Phosphorylation } \\
\text { Sites }\end{array}$ & Secretion \\
\hline BGH00219*** & 31.9 & GH17 motif & $\begin{array}{c}2 \\
(185 ; 204)\end{array}$ & 30 & Signal Peptide \\
\hline BGH00220*** & 32 & GH17 motif & $\begin{array}{c}1 \\
(274)\end{array}$ & 23 & Signal peptide \\
\hline BGH00734** & 58.5 & $\begin{array}{c}\text { GH17 motif } \\
\text { Threonine-rich } \\
\text { region }\end{array}$ & $\begin{array}{c}2 \\
(85 ; 530)\end{array}$ & 63 & Signal peptide \\
\hline BGH00736** & 85.5 & GH17 motif & $\begin{array}{c}4 \\
(191 ; 460 ; 512 ; \\
678)\end{array}$ & 91 & Unconventional \\
secretion
\end{tabular}

* Protein identification numbers were retrieved from www.blugen.org.

** Nucleotide and amino acid sequence obtained from Dr. Patrick Schweizer

*** Nucleotide and amino acid sequence obtained from https://www.ebi.ac.uk/ena

\subsubsection{Analysis of the potential role of $\mathrm{Bgh}$ GH17 genes in pathogenicity}

Host-induced gene silencing (HIGS) represents a "tool to address gene function in obligate biotrophic fungi" and is based on RNA interference (RNAi) (Nowara et al., 2010). RNAi molecules that target $B g h$ transcripts are expressed in planta and may be exchanged between the host and the established $B g h$ haustorium. The uptake of the RNAi construct by Bgh leads to silencing of the fungal target gene and allows to analyse the gene function (Nowara et al., 2010). In order to analyse the contribution of specific genes to invasiveness, a first HIGS experiment was performed by our collaborators at the IPK Gatersleben (unpublished data; Dr. Patrick Schweizer, personal communication). These preliminary analyses suggested that silencing of Bgh06298 and Bgh05070 resulted in an enhanced penetration 
rate indicating that these genes have a negative impact on pathogenicity and might either be directly recognized by the plant or involved in the generation of DAMPs. Although the penetration rate was not significantly increased, these candidates were excluded from further analysis. On the contrary, silencing of Bgh06777 or Bgh00734 might lead to a reduced penetration rate leading to the suggestion that these genes are involved in pathogenicity (unpublished data; Dr. Patrick Schweizer, personal communication). Thus, the GH17 family members BGH06777, BGH00219, BGH00220, BGH00734 and BGH00736 were chosen for further functional characterization.

\subsubsection{Recombinant production of Bgh GH17 proteins in Pichia pastoris}

Several heterologous expression systems are available that can be used to produce recombinant proteins, e.g. Escherichia coli, Pichia pastoris or Saccharomyces cerevisiae. In contrast to E. coli, $P$. pastoris and $S$. cerevisiae offer the advantage of eukaryotic protein processing, protein folding as well as posttranslational modifications. As $P$. pastoris in contrast to $S$. cerevisiae does not hyperglycosylate proteins (Daly and Hearn, 2005; Gomes et al., 2016), P. pastoris was selected as heterologous expression system for the selected Bgh genes Bgh00219, Bgh00220, Bgh06777, $B g h 00734$ and Bgh00736. The genes Bgh00220 and Bgh06777 without the signal peptide were amplified from cDNA and cloned into the pPICZa expression vector with a C-terminal His and Myc tag. pPICZa expression vectors for Bgh00219, Bgh00734 and Bgh00736 were synthesized by BioBasic (Markham, Ontario, Canada). The sequence for the expression vector for Bgh00219 is based on the cDNA sequence of Bgh00219 without the signal peptide, while the sequence for the expression vector of Bgh00734 is based on the cDNA sequence with the signal peptide. For the synthesis of the Bgh00736 expression vector, cDNA with the signal peptide was used as a template. The expression vectors for all genes were successfully transformed into $P$. pastoris $\mathrm{X}-33$ cells. Three to four clones were tested for the presence of either BGH00219, BGH00220, BGH00734, BGH00736 or BGH06777 in a small scale expression test using four different conditions. The clones were either grown at $16^{\circ} \mathrm{C}$ or $25^{\circ} \mathrm{C}$ and either $1 \%$ or $3 \%$ methanol were added every $24 \mathrm{~h}$. For both BGH00219 and BGH00220 a distinct band at $34 \mathrm{kDa}$ (with Myc and His-tag) was expected. However, no signal for either BGH00219 or BGH00220 was detected at the expected size. Instead, all clones transformed with the plasmid encoding for Bgh00219 exhibited several signals at various sizes while clones expressing Bgh00220 showed either several signals or no signal at all (Figure S1 A and B). For BGH00734 a signal migrating at $66 \mathrm{kDa}$ (with Myc and His-tag) should be detected, while a signal at $87 \mathrm{kDa}$ should be visible for BGH00736 (with Myc and His-tag). As for BGH00219 and BGH00220, no distinct signals at the expected sizes could be detected for either BGH00734 or BGH00736 (Figure $\mathrm{S} 1 \mathrm{C}$ and $\mathrm{D}$ ). This indicates that none of the tested clones can be used to purify these four proteins. The detected signals might correspond to the respective proteins that were proteolytically cleaved, posttranslationally modified or might represent contaminations. For BGH06777 a signal at about $33 \mathrm{kDa}$ (with Myc- and His tag) was expected. Fortunately, all tested clones expressed Bgh06777 with clone \#17 showing the highest expression while growing at $16^{\circ} \mathrm{C}$ and addition of $1 \%$ methanol 
(Figure S1 E). Consequently, this clone was chosen for the scale-up expression to produce more of the respective protein. The recombinant protein was purified via FPLC, following size exclusion and the purification yield was $12 \mathrm{mg} / \mathrm{L}$. According to SDS PAGE, purified BGH06777 migrated at a size of $35 \mathrm{kDa}$ (calculated: $35 \mathrm{kDa}$ corresponding to BGH06777 with N-linked oligosaccharides containing 14 mannose residues and $\mathrm{N}$-terminal Myc and His-tag). To verify the glycosylation, recombinant BGH06777 was incubated with PNGase $F$ which is an amidase that cleaves $\mathrm{N}$-linked oligosaccharides from glycosylated proteins (Plummer et al., 1984). Upon PNGase F treatment, BGH06777 migrated at $33 \mathrm{kDa}$ (calculated: $32.967 \mathrm{kDa}$ corresponding to BGH06777 with N-terminal Myc- and His-tag) indicating that P. pastoris glycosylated BGH06777 (Figure S2). In order to further verify the protein mass of recombinant BGH06777, mass spectrometry was performed. Several masses between $35169 \mathrm{Da}$ and $36873 \mathrm{Da}$ were identified during the analysis of glycosylated BGH06777 (Figure 4 A). The identified masses correspond to recombinant BGH06777 with N-linked oligosaccharides of varying length (11 - 20 mannose residues) and BGH06777 with phosphorylated $\mathrm{N}$-linked oligosaccharides of varying length (13 - 18 mannose residues). Upon treatment with PNGase F, the major mass identified was 32977 Da which corresponds to the deglycosylated form of recombinant BGH06777 (Figure $4 \mathrm{~B}$ ). In the following biochemical characterization, the glycosylated form of recombinant BGH06777 was used. 
A

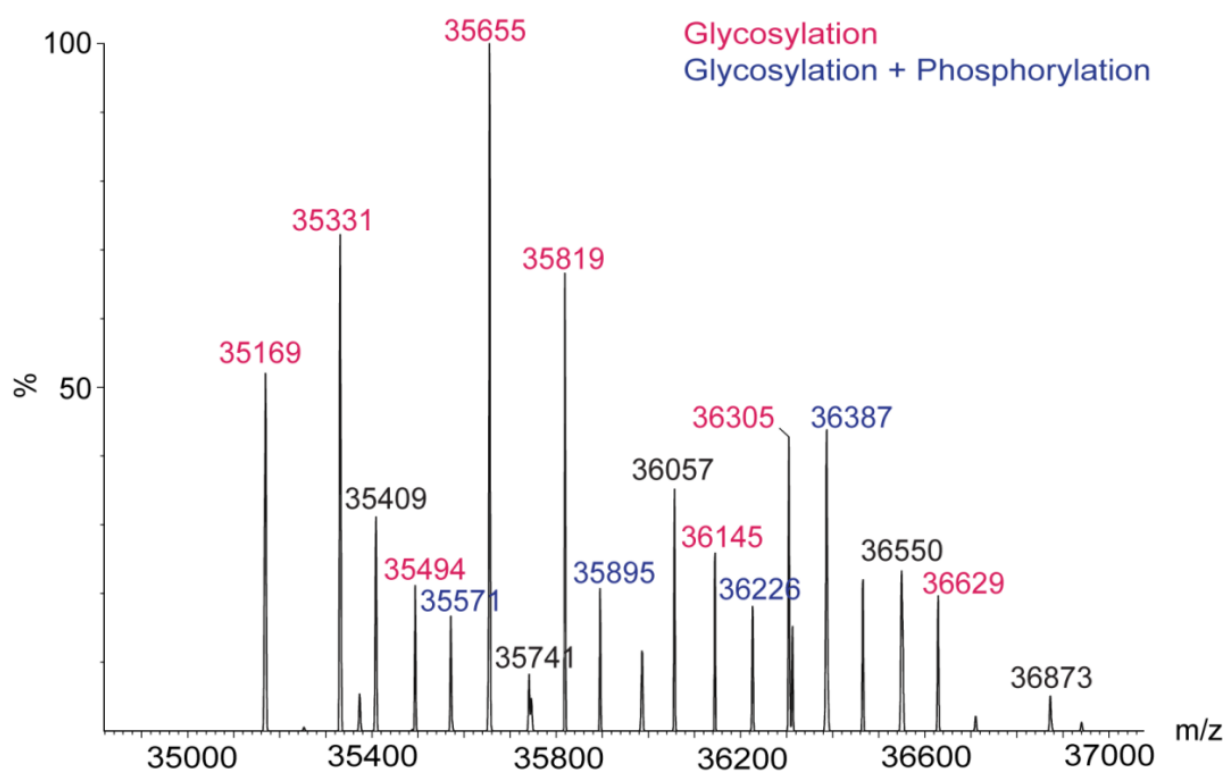

B

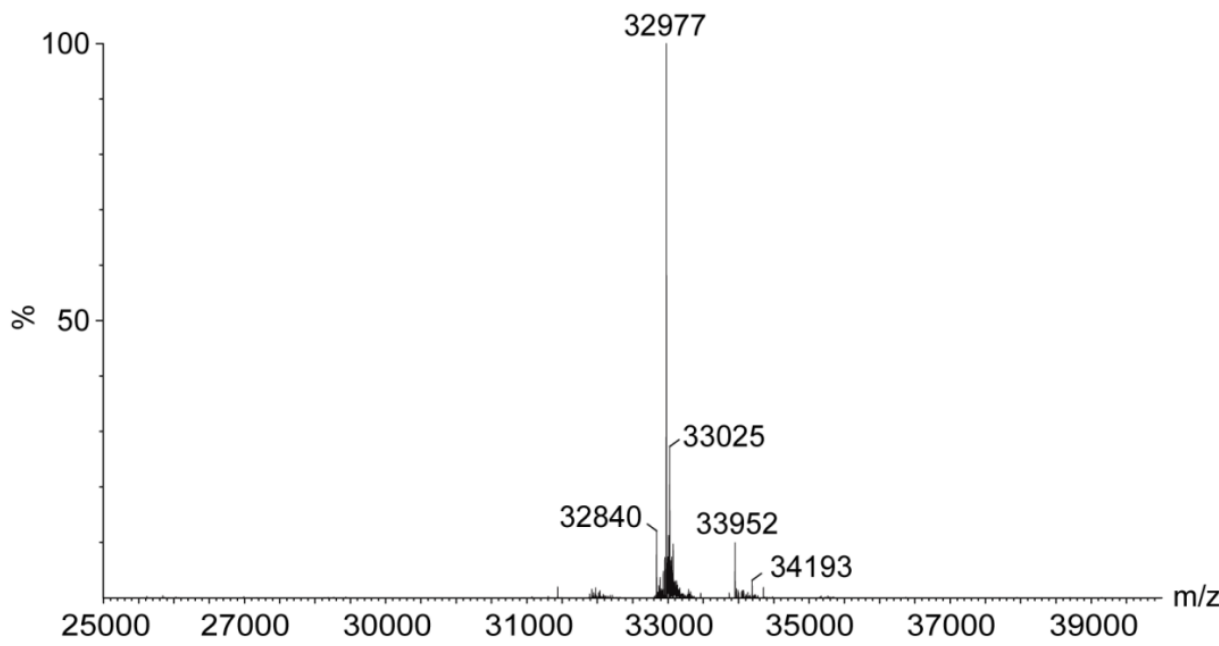

Figure 4. Mass spectra of recombinant BGH06777. (A) Mass spectra of glycosylated and phosphorylated BGH06777. Protein mass of purified BGH06777 was verified by analyzing the mass via mass spectrometry. Pink lettering indicates masses of $\mathrm{N}$-glycosylated BGH06777 with varying length of mannose residues (11 - 20). Blue lettering indicates masses of $\mathrm{N}$-glycosylated BGH06777 with varying length of mannose residues (13 - 18) and phosphorylation. (B) Mass spectra of deglycosylated BGH06777. To verify glycosylation of purified BGH06777, BGH06777 was treated with PNGase F overnight. Subsequently, the protein mass by determined via mass spectrometry. 


\subsection{6 $\beta$-1,3-glucan oligosaccharides are substrates of BGH06777}

GH family 17 includes enzymes that hydrolyse $\beta$-1,3-glycosidic linkages in $\beta$-1,3-glucans (E.C.3.2.1.39, E.C.3.2.1.58) or $\beta$-1,3-glycosidic linkages in fungal $\beta-1,3 ; 1,6$-glucans if a region of unbranched $\beta$-1,3-glucan residues is available. Furthermore, a $\beta-1,3 ; 1,4$-glucan endo-hydrolase belonging to $\mathrm{GH} 17$ was shown to hydrolyse $\beta-1,4$-glycosidic linkages in plant $\beta-1,3 ; 1,4-$ glucans (E.C.3.2.1.73) if the $\beta-1,4-$ glycosidic linkage is located at the reducing end (Woodward and Fincher, 1982; Hrmova and Fincher, 1993; Carbohydrate Active Enzymes database, http://www.cazy.org/, Lombard et al., 2014). Based on the substrate specificities known for $\mathrm{GH} 17$ family members, recombinant BGH06777 was tested for activity on $\beta-1,3$-glucan oligosaccharides. Therefore, recombinant BGH06777 was incubated with $\beta$-1,3-glucan oligosaccharides of varying length (6-3) at room temperature in MES buffer $(\mathrm{pH}=5)$ and the hydrolysis products were analysed by highperformance anion exchange chromatography with pulsed amperometric detection (HPAEC-PAD). HPAEC-PAD represents a highly sensitive method for oligosaccharide separation. The hydroxyl groups are ionized under strongly basic conditions and retain at the anion exchange column. The ionized oligosaccharides can be released at a certain retention time depending on e.g. the molecular size, the number of hydroxyl groups and the structure of the oligosaccharide. The mobile phase is composed of the oligosaccharide dissolved in water. To elute the oligosaccharides from the column a constant concentration of sodium hydroxide is used with an increasing concentration of sodium acetate over time. Sodium acetate is a stronger eluent compared to sodium hydroxide and is used to elute longer oligosaccharides or acidic sugars. To detect the carbohydrate, an electronic current that is generated upon oxidation of the oligosaccharide on a gold-electrode is measured (Corradini et al., 2012).

Hydrolysis of laminarihexaose (G3G3G3G3G3G) yielded in the formation of laminaritetraose (G3G3G3G) and laminaribiose (G3G) (Figure 5). Upon 12h incubation of laminarihexaose (G3G3G3G3G3G) with BGH06777, laminarihexaose (G3G3G3G3G3G) was completely degraded (Figure 5, blue line). The resulting oligosaccharides from laminaripentaose (G3G3G3G3G) degradation were laminaritriose (G3G3G) and laminaribiose (G3G). As observed for laminarihexaose, laminaripentaose was completely degraded upon 12h incubation with BGH06777 (Figure 5, blue line). The degradation of laminaritetraose (G3G3G3G) resulted in the formation of laminaribiose (G3G), however, laminaritetraose was not completely degraded upon $12 \mathrm{~h}$ incubation with the enzyme (Figure 5 , blue line). The $\beta$-1,3-oligosaccharide laminaritriose was not degraded by BGH06777 (Figure 5). These results indicate that recombinant BGH06777 uses $\beta$-1,3-glucan oligosaccharides of a minimum length of four glucose monomers as substrates. 

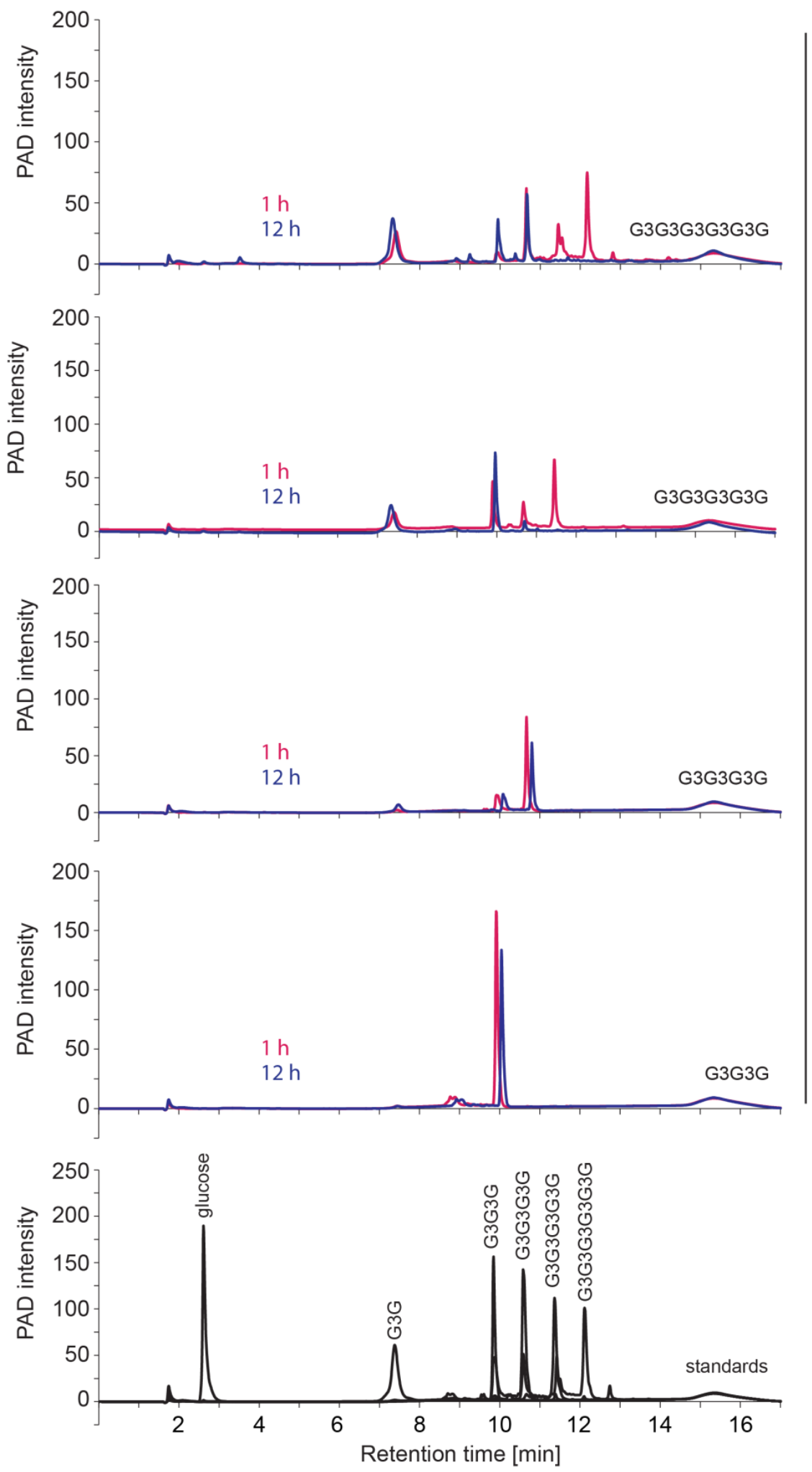

Figure 5. HPAEC-PAD chromatograms of $\beta$-1,3-glucan oligosaccharide hydrolysis by BGH06777. $1 \mu \mathrm{g} \mathrm{m}^{-1} \mathrm{BGH} 06777$ was incubated with $0.05 \mathrm{mM}$ laminarihexaose (G3G3G3G3G3G), laminaripentaose (G3G3G3G3G), laminaritetraose (G3G3G3G) and laminaritriose (G3G3G) in $50 \mathrm{mM}$ MES buffer $(\mathrm{pH}=5)$ at room temperature. Upon $1 \mathrm{~h}$ (pink line) or $12 \mathrm{~h}$ (blue line) incubation, the hydrolysis products were analysed by HPAEC-PAD. As standards, the respective $\beta-1,3-$ glucan oligosaccharides and glucose were included. 


\subsubsection{Optimal temperature and pH conditions for BGH06777}

The activity of enzymes can be affected by changing environmental conditions, e.g. $\mathrm{pH}$ or temperature. Each enzyme has a $\mathrm{pH}$ and temperature optimum at which its activity rate is the highest (Robinson, 2015). To determine the optimal temperature of BGH06777, recombinant BGH06777 was incubated with laminarihexaose at different temperatures $\left(5^{\circ} \mathrm{C}-78^{\circ} \mathrm{C}\right)$ in $50 \mathrm{mM}$ Citrate buffer $(\mathrm{pH}=5)$ and the amount of the generated product laminaribiose was measured to determine the activity rate using HPAEC-PAD. Recombinant BGH06777 was active within a temperature range of $15^{\circ} \mathrm{C}$ to $57^{\circ} \mathrm{C}$ with the highest activity rate observed in a range from $25^{\circ} \mathrm{C}$ to $51^{\circ} \mathrm{C}$ (Figure $6 \mathrm{~A}$ ).

In order to determine the optimal $\mathrm{pH}$, recombinant $\mathrm{BGH} 06777$ was incubated with its substrate laminarihexaose in different buffers ranging from $\mathrm{pH} 3-10$ at $30^{\circ} \mathrm{C}$. The activity rate was measured as the amount of generated laminaribiose via HPAEC-PAD. Recombinant BGH06777 was active in a pH range from 4.5 to 8 . The optimal $\mathrm{pH}$ for recombinant $\mathrm{BGH} 06777$ was shown to be at 5.5 (Figure $6 \mathrm{~B}$ ).
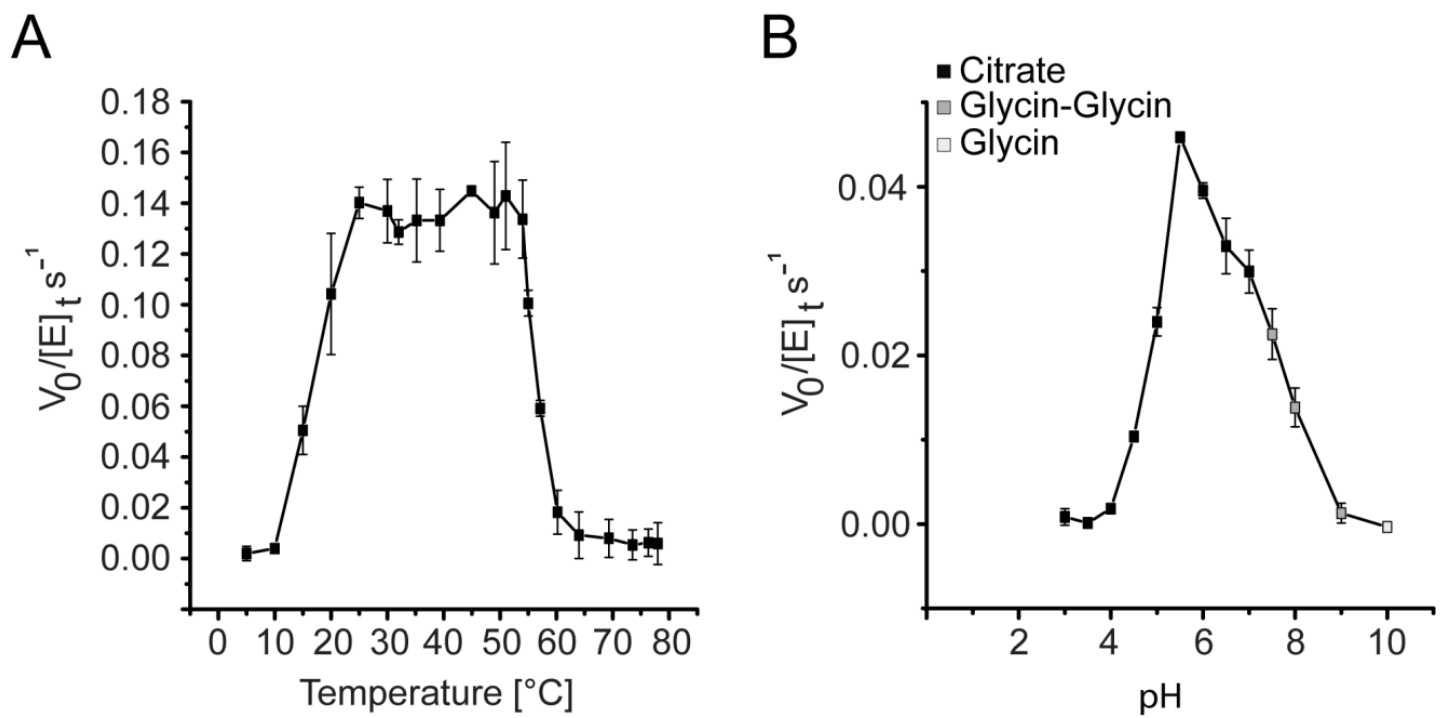

Figure 6. Temperature and pH profile of recombinant BGH06777. (A) Temperature optimum of BGH06777. For the temperature profile, $2 \mu \mathrm{g} \mathrm{ml}^{-1} \mathrm{BGH} 06777$ were incubated in $50 \mathrm{mM}$ Citrate buffer $(\mathrm{pH}=5)$ with $1 \mathrm{mM}$ laminarihexaose. The reaction was incubated for $15 \mathrm{~min}$ or $1 \mathrm{~h}$ at the respective temperature. Upon deactivation of BGH06777, the enzymatic rate was measured as increase in product peak via HPAEC-PAD. Error bars represent standard deviation. (B) $\mathrm{pH}$ optimum for BGH06777. To determine the $\mathrm{pH} p r o f i l e$, $2 \mu \mathrm{g} \mathrm{ml}^{-1}$ BGH067777 were incubated with $10 \mathrm{mM}$ laminarihexaose in $50 \mathrm{mM}$ buffer with different $\mathrm{pH}$ ranging from 3-10. The following buffers were used: Citrate buffer from $\mathrm{pH} 3-7$, Glycin-Glycin buffer from $\mathrm{pH} 7.5$ to 9 and Glycin buffer for $\mathrm{pH} 10$. The enzymatic rate was measured as increase in product peak over time. Error bars represent standard error. 


\subsubsection{Michaelis-Menten parameters of BGH06777}

To characterize enzymes regarding activity rates and the influence of changing conditions on enzymatic activity, kinetic models are used. The most commonly used model is the Michaelis-Menten model. This model is based on the assumption that an enzyme ( $E$ ) forms an unstable enzyme-substrate complex (ES) with its substrate (S) followed by decay to enzyme and product $(P)$ (Michaelis and Menten, 1913) (Figure 7 A). Under three further assumption, that 1) the substrate concentration is much higher than the enzyme concentration, 2) only initial velocities are taken into account and thus, product formation does not influence the reaction and 3) the speed of the formation of enzyme-substrate complex is equal to the decay of enzyme-substrate complex, the enzymatic rate can be described in a mathematical equation as relation of the product formation rate and substrate concentration (Figure $7 \mathrm{~B}$ ), where $\mathrm{v}$ is the initial velocity, $\mathrm{k}_{\text {cat }}$ (the turnover number) describes the number of substrate molecules that is converted to product per enzyme site per time, $[E]_{0}$ is the initial enzyme concentration, while the Michaelis-Menten constant $\left(\mathrm{K}_{\mathrm{M}}\right)$ represents the substrate concentration that yield in half-maximal velocity (Michaelis and Menten, 1913). This equation can be used to calculate enzyme kinetics from enzyme reactions with varying substrate concentrations. Furthermore, the ratio of $k_{c a t}$ to $K_{M}$ can be used to measure catalytic efficiency (Eisenthal et al., 2007).

A

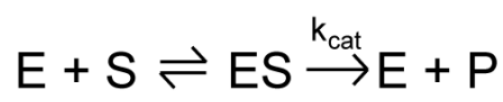

B

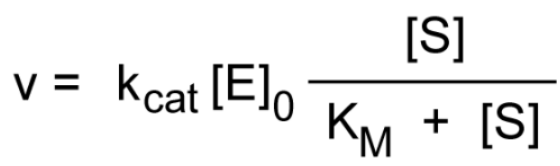

Figure 7. Michaelis-Menten model for an enzymatic reaction and the resulting equation. (A) Model for an enzymatic reaction. Enzyme (E) forms a complex (ES) with its substrate (S) followed by the release of a product $(P)$ and a free enzyme $(E)$. The double arrow indicates that the formation of the enzyme-substrate complex (ES) is reversible. (B) Michaelis-Menten-Equation with $v$ - initial velocity, $\mathrm{k}_{c a t}-$ number of substrates converted to product per time, $(E)_{0}$ - enzyme concentration, [S] - substrate concentration, $\mathrm{K}_{\mathrm{M}}$ - substrate concentration with which half-maximal velocity is achieved.

To identify Michaelis-Menten kinetics for recombinant BGH06777 for the two substrates laminarihexaose (G3G3G3G3G3G) and laminaripentaose (G3G3G3G3G), the enzyme was incubated with the respective substrates in eight different concentrations and the initial velocities were measured over time as increase in product peak (laminaribiose) via HPAEC-PAD. The obtained initial velocities were plotted against the substrate concentration and Michaelis-Menten parameters ( $k_{\text {cat }}$ and $K_{M}$ ) were determined by non-linear fit using the Michaelis-Menten model in the software OriginPro (Figure 8, Table 19). 
A

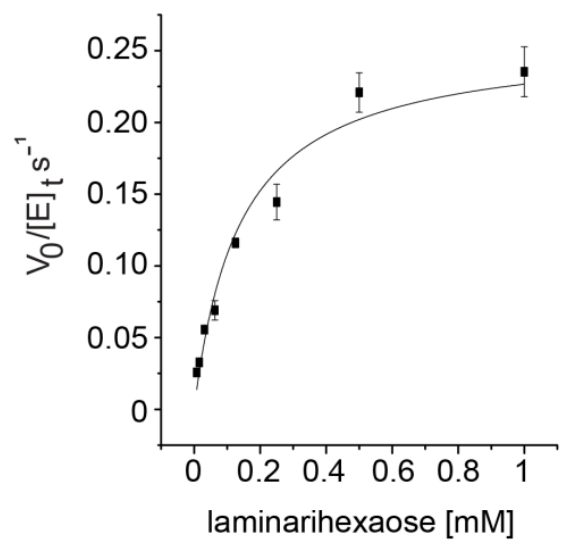

B

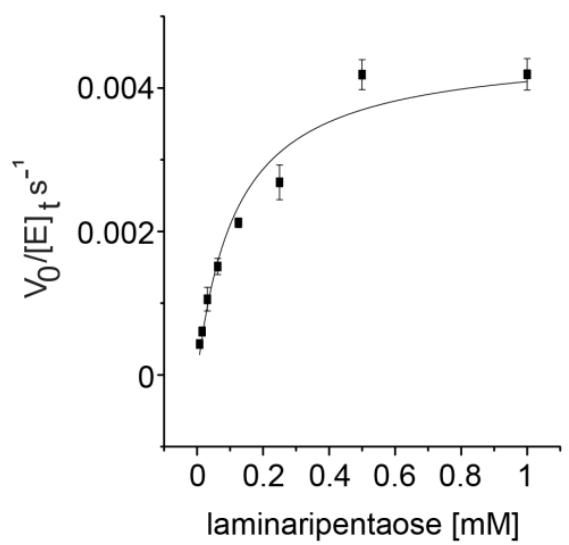

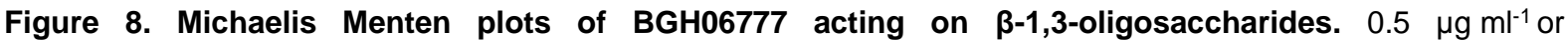
$5 \mathrm{\mu g} \mathrm{ml}^{-1} \mathrm{BGH} 06777$ were incubated with laminarihexaose (A) or laminaripentaose (B) in $200 \mathrm{mM}$ Citrate Buffer $(\mathrm{pH}=5.5)$ at $30^{\circ} \mathrm{C}$. Initial velocities rates were measured for eight different substrate concentrations as increase in product peak over time via HPAEC-PAD. The initial velocities were plotted against substrate concentrations and Michaelis-Menten parameters were determined via non-linear fit using the Michaelis-Menten model in Origin Pro. Error bars represents standard errors.

Recombinant BGH06777 hydrolysed laminarihexaose with the highest $\mathrm{k}_{\mathrm{cat}}$ values of the two tested substrates at $\mathrm{a} \mathrm{pH}$ of 5.5 and $30^{\circ} \mathrm{C}$ (Table 19). Under these conditions, laminaripentaose was hydrolysed less efficiently with a 5.6 fold lower $k_{\text {cat }}$ value as observed for laminarihexaose (Table 19). The $\mathrm{K}_{\mathrm{M}}$ value of $\mathrm{BGH06777}$ for laminarihexaose was higher compared to the $\mathrm{K}_{\mathrm{M}}$ value obtained for laminaripentaose (Table 19). This indicates that a higher laminarihexaose concentration is required to result in the maximum reaction velocity in comparison to degradation of laminaripentaose. However, laminarihexaose was hydrolysed more efficiently than laminaripentaose as the $\mathrm{K}_{\text {cat }} / \mathrm{K}_{M}$ value obtained for laminarihexaose hydrolysis was 4.8-times higher than for laminaripentaose hydrolysis (Table 19).

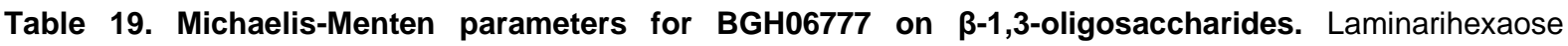
(G3G3G3G3G3G) and laminaripentaose (G3G3G3G3G). Errors indicate errors in fitting the data to the MichaelisMenten equation.

\begin{tabular}{cccc}
\hline Substrate & $\mathbf{k}_{\text {cat }}\left[\mathbf{s}^{-1}\right]$ & $\mathbf{K}_{\mathbf{M}}[\mathbf{m M}]$ & $\mathbf{k}_{\text {cat }} / \mathbf{K}_{\mathbf{M}}\left[\mathrm{mM}^{-1} \mathbf{s}^{-1}\right]$ \\
\hline G3G3G3G3G3G & $0.25809 \pm 0.02611$ & $0.13887 \pm 0.03236$ & 1.858 \\
\hline G3G3G3G3G & $0.04577 \pm 0.0039$ & $0.11929 \pm 0.02416$ & 0.3836 \\
\hline
\end{tabular}




\subsubsection{BGH06777 has a -4/+2 binding/hydrolysis mode}

The active site of an enzyme is the site where the substrate binds to the enzyme and gets hydrolysed. Active sites of GH can be designated to negative subsites (-n) towards the non-reducing end of the sugar and positive subsites $(+n)$ towards the reducing end of the sugar with $n$ being the number of either positive or negative subsites. The hydrolysis of oligosaccharides occurs between the -1 and +1 subsite (Davies et al., 1997). During the hydrolysis, a new reducing end is generated due to the incorporation of a hydroxyl group to a carbon of the glycosyl intermediate in the -1 subsite. The incorporated hydroxyl group is derived from surrounding water (Varrot et al., 2001). In the presence of ${ }^{18} \mathrm{O}$ labelled water, ${ }^{18} \mathrm{OH}$ is incorporated to the newly formed reducing end which results in an increase of mass by two units $(M+2)$ of the product bound to the negative subsites. The increase in mass can be monitored using mass spectrometry. Thus, the cleavage point of an oligosaccharide and therefore, the number of positive and negative subsites of an enzyme, can be determined by analysing the obtained hydrolysis products in the presence of ${ }^{18} \mathrm{O}$ labelled water via mass spectrometry (Schagerlöf et al., 2009).

To determine the number of negative and positive subsites of BGH06777, BGH06777 in citrate buffer $(\mathrm{pH}=5.5)$ was incubated with laminarihexaose in $\mathrm{H}_{2}{ }^{18} \mathrm{O}$ and the ${ }^{18} \mathrm{O}$ incorporation was subsequently analysed using mass spectrometry. Hydrolysis products of laminarihexaose by BGH06777 are laminaritetraose (G3G3G3G) and laminaribiose (G3G). The M+2 peak of laminaribiose (G3G) did not increase compared to normal abundance of laminaribiose (G3G), while the $M+2$ peak of laminaritetraose (G3G3G3G) increased. This is further indicated by ${ }^{18} \mathrm{O}$ labelling of $91 \%$ of laminaritetraose (G3G3G3G) (Figure 9, A and B, Table 20). The exclusive labelling of laminaritetraose (G3G3G3G) reveals the presence of four negative and two positive subsites and a $-4 /+2$ hydrolysis mode. 


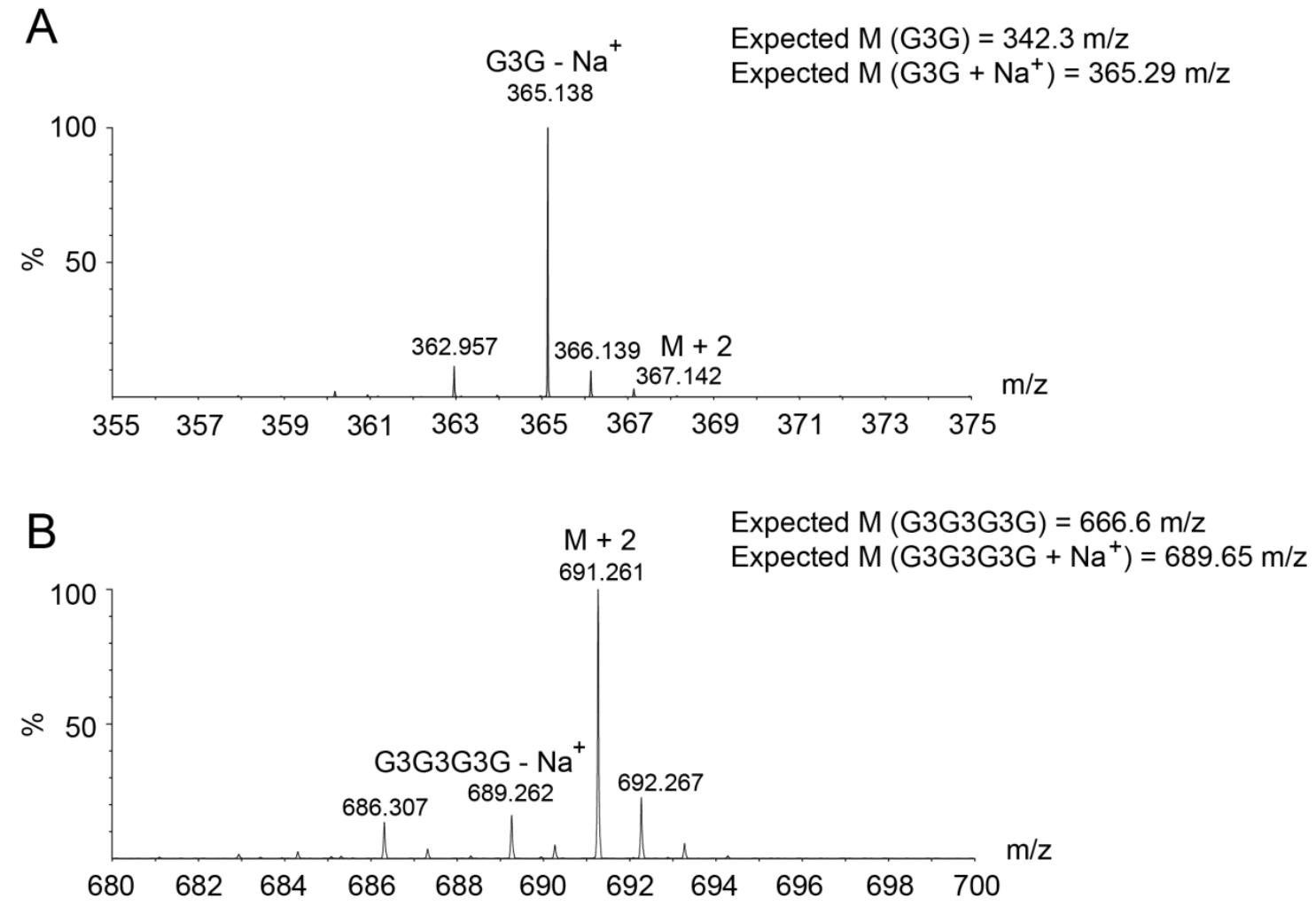

Figure 9. Mass spectrometric analysis of the hydrolysis of laminarihexaose in presence of $\mathrm{H}_{2}{ }^{18} \mathrm{O}$ by BGH06777. (A) Mass spectra of laminaribiose (G3G) and (B) mass spectra of laminaritetraose (G3G3G3G). BGH067777 was incubated with laminarihexaose (G3G3G3G3G3G) in presence of $\mathrm{H}_{2}{ }^{18} \mathrm{O}$. Upon incubation, the masses of the products laminaribiose (G3G) and laminaritetraose (G3G3G3G) were analysed via mass spectrometry. For each spectrum, the expected masses of the products are given. An increase in $M+2$ indicates ${ }^{18} \mathrm{O}$ incorporation.

Table 20. Degree of ${ }^{18} \mathrm{O}$ labelled laminaripentaose (G3G3G3G) and laminaribiose (G3G) derived from hydrolysis of laminarihexaose (G3G3G3G3G3G) by BGH06777. The percentage of labelling was calculated from peak integrations and corrected for $\mathrm{H}_{2}{ }^{16} \mathrm{O}$ derived from substrate and enzyme solution.

\begin{tabular}{cc}
\hline Substrate & \\
\hline Product & \\
\hline G3G3G3G3G3G & \\
\hline G3G3G3G & $91.16 \pm 3.9$ \\
\hline G3G & $2.33 \pm 3.9$ \\
\hline
\end{tabular}

To summarize, the Bgh GH17 protein BGH06777 employs a -4/+2 binding/hydrolysis mode and is able to hydrolyse $\beta$-1,3-glucan oligosaccharides with a minimum length of four glucose monomers. Furthermore, the optimal conditions at which the protein shows the highest activities were identified. 


\subsection{Identification and analysis of novel cell-wall derived DAMPs}

\subsubsection{Screen to identify cell-wall derived DAMPs in Arabidopsis}

The plant cell wall composed of proteins, cellulose, hemicelluloses and pectic polysaccharides provides not only structure to the plant cell but is also a physical barrier to microbial invasion. Furthermore, it represents a potential source for DAMPs. In order to overcome the plant cell wall, pathogens secrete several CWDEs that are able to hydrolyse specific cell wall components (Malinovsky et al., 2014). A variety of cell-wall derived oligosaccharides is likely to be generated by fungal CWDEs which might be perceived by the plant and consequently, lead to the activation of plant immune responses. These immune responses include the influx of $\mathrm{Ca}^{2+}$ ions into the cytosol, the generation of ROS and the activation of MAPK (Bigeard et al., 2015).

A second aim of this study was to identify new cell wall derived DAMPs in the dicot model plant Arabidopsis. To this end, 37 cell-wall derived poly- and oligosaccharides that are present in the cell wall of the dicot model plant Arabidopsis were purchased from Megazyme (Bray, Ireland). These substances included a cellulose derivative, namely cellohexaose, hemicelluloses e.g. arabinofuranosyl-xylotetraose, pectic substances e.g. rhamnogalacturonan I, $\beta-1,3$-glucans e.g. laminarihexaose. All 37 substances (Table 7) were analysed regarding their ability to induce immune responses with three different methods. First, the influx of $\mathrm{Ca}^{2+}$ ions was analysed by using Arabidopsis Col-0 seedlings that express the calcium sensor protein aequorin. Different Arabidopsis ecotypes were shown to have a distinct receptor repertoire and might therefore be either sensitive or insensitive towards a specific MAMP. MAMP-sensitive and MAMP-insensitive accessions can be used to identify the respective receptor with a map-based cloning strategy (Jehle et al., 2013b; Zhang et al., 2014). To possibly identify DAMP-sensitive and DAMP-insensitive accessions, ROS burst generation as well as MAPK activation upon treatment with all substances was tested in Col-0, Ws-0 and Ws-4. 33 of the ordered substances did not induce PTI responses (Figure S3, Figure S4, Figure S5, Figure S6, Figure S7, Figure S8, Figure S9), while four carbohydrates could be identified that at least slightly induced the immune response.

The cellulose derivative cellohexaose was not able to induce the generation of ROS, however, did induce a slight influx of $\mathrm{Ca}^{2+}$ in Col-0 and activation of MAPK in Col-0, Ws-0 as well as Ws-4 (Figure S10, Figure S11) indicating a potential role as DAMP. In 2017, the group of Shauna Somerville released a publication showing that cellobiose acts as DAMP (de Azevedo Souza et al., 2017), thus, confirming our results but excluding further analysis of cellohexaose as a DAMP.

Furthermore, the hemicellulose derivative xylohexaose could neither induce the generation of ROS in Col-0, Ws-0 and Ws-4 nor the influx of $\mathrm{Ca}^{2+}$ in Col-0 (Figure S12, Figure S13 A). However, xylohexaose treatment induced MAPK activation in Col-0, Ws-0 and Ws-4 (Figure S13 B). However, the obtained results for xylohexaose were not robust and thus, this oligosaccharide was also excluded from further analysis.

The $\beta$-1,3-glucan oligosaccharides laminarihexaose, laminaripentaose, laminaritetraose, laminaritriose and laminaribiose were not able to induce either the influx of $\mathrm{Ca}^{2+}$ in Col-0 or the ROS burst in Col-0, Ws-0 and Ws-4 (Figure S14, Figure S15 A). The activation of MAPK could only slightly 
be induced by laminarihexaose and laminaripentaose in all three tested ecotypes (Figure S15 B). Since the tested $\beta-1,3$-glucans did only slightly induce the response in one of three assays, these substances were excluded from further analysis. In 2018, however, the research group of Antonio Molina could show that laminarihexaose acts as a robust elicitor of immune responses in Arabidopsis (Mélida et al., 2018). The discrepancies in the results might be explained with the different concentrations used in the assays. Whilst $250 \mu \mathrm{M}$ of the oligosaccharides were used by the research group of Antonia Molina, only $10 \mu \mathrm{M}$ to $100 \mu \mathrm{M}$ were used in this study.

In conclusion, none of the so far tested poly- and oligosaccharides will be used for further analysis as they were either not robustly inducing immune responses or were identified as DAMPs by other research groups during the course of this project.

\subsubsection{Analysis of the DAMP capacity of MLGs in barley}

In the collection of cell-wall derived poly- and oligosaccharides purchased from Megazyme, one MLG tetramer, namely cellotriosyl-glucose, one MLG tetramer mixture, called cellobiosyl-cellobiose plus glucosyl-cellotriose, and two MLG trimers, namely cellobiosyl-glucose and glucosyl-cellobiose were included. MLGs are composed of glucose monomers that are connected via both $\beta-1,3-$ and $\beta$-1,4-linkages (Burton and Fincher, 2009). The MLG tetramer, the MLG tetramer mixture and the two trimers of our collection differ in the position of the $\beta-1,3$-linkage. For ease of understanding, the MLG oligomers will be abbreviated and the positions of $\beta-1,3$-linkage highlighted by color from now on, so that that the tetramer cellotriosyl-glucose is represented by G4G4G3G, cellobiosyl-cellobiose plus glucosyl-cellotriose as G4G3G4G + G3G4G4G and the two trimers cellobiosyl-glucose as G4G3G and glucosyl-cellobiose as $G 3 G 4 G$, with $G$ standing for glucose and 3 and 4 representing the $\beta-1,3-$ and $\beta-1,4$-linkages, respectively.

MLGs are abundant cell wall components in monocots e.g. in the crop plant barley but not in dicots. In first leaves of six-day old barley plants e.g. MLGs can be found in the cell walls of epidermal as well as in palisade and spongy mesophyll cells (Trethewey and Harris, 2002). Since MLGs are not present in the cell wall of Arabidopsis and thus, cannot act as DAMP, their DAMP activity was tested in barley. Therefore, the generation of ROS upon treatment with MLG oligomers in barley leaves was analysed with a luminol-based assay. Upon treatment with the MLG tetramer G4G4G3G, the tetramer mixture G4G3G4G + G3G4G4G and the two trimers G3G4G and G4G3G, the generation of ROS was induced in barley. The ROS burst induced by the tetramer G4G4G3G, however, was less pronounced in comparison to the ROS burst induced by the G4G3G4G + G3G4G4G mixture (Figure $10 \mathrm{~A}$ ). Besides the generation of ROS, the activation of MAPK is a typical response towards MAMPs and was shown to be triggered in barley upon chitin and flg22 elicitation (Scheler et al., 2016). To further verify the ability of MLGs to trigger immune responses in barley, the activation of MAPK upon MLG treatment was analysed via Western Blot using the p44/42 antibody which specifically detects phosphorylated MAPKs. As observed before for chitin and flg22, one signal could predominantly be detected in immunoblot analysis (Scheler et al., 2016) (Figure $10 \mathrm{~B}$ ). The MLG tetramer mixture 
G4G3G4G + G3G4G4G as well as the trimer G4G3G induced MAPK phosphorylation, while G4G4G3G only slightly induced the activation of MAPK (Figure $10 \mathrm{~B}$ ). The findings that MLG treatment induces the generation of ROS as well as the activation of MAPK in barley indicate that barley is able to perceive MLGs and consequently, immune responses are activated.

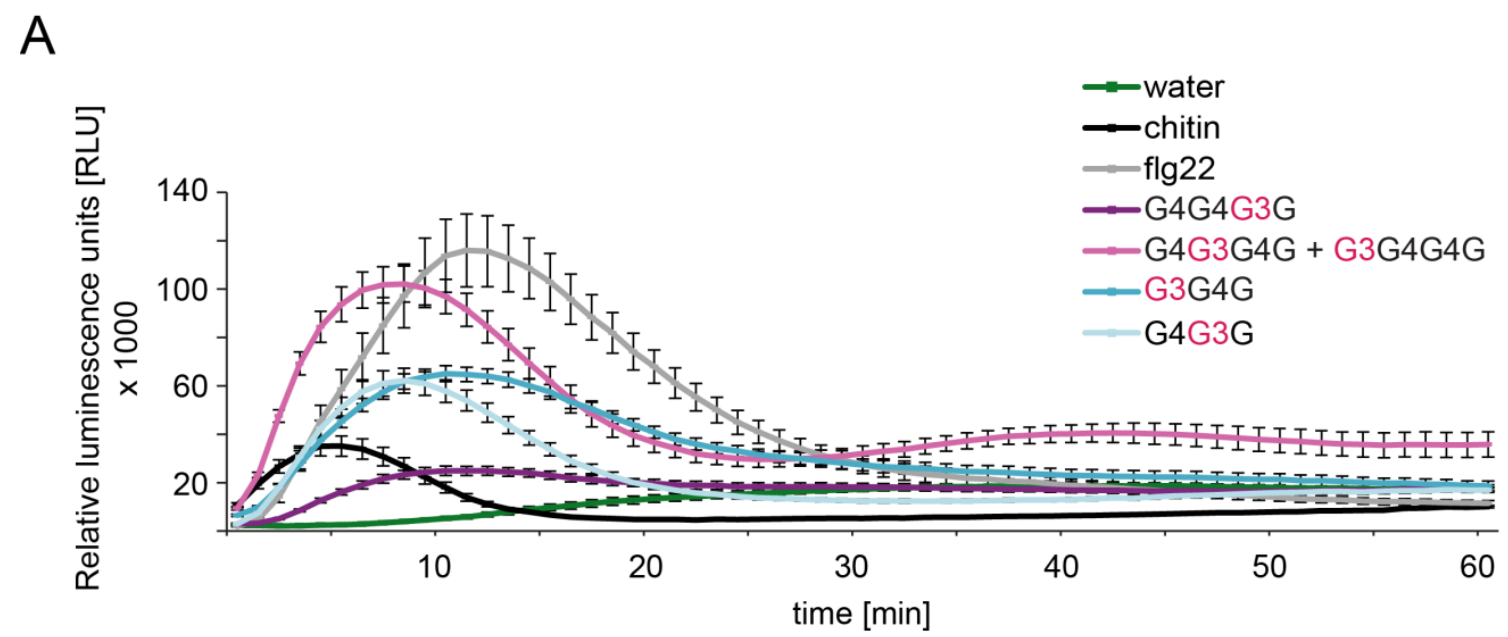

B

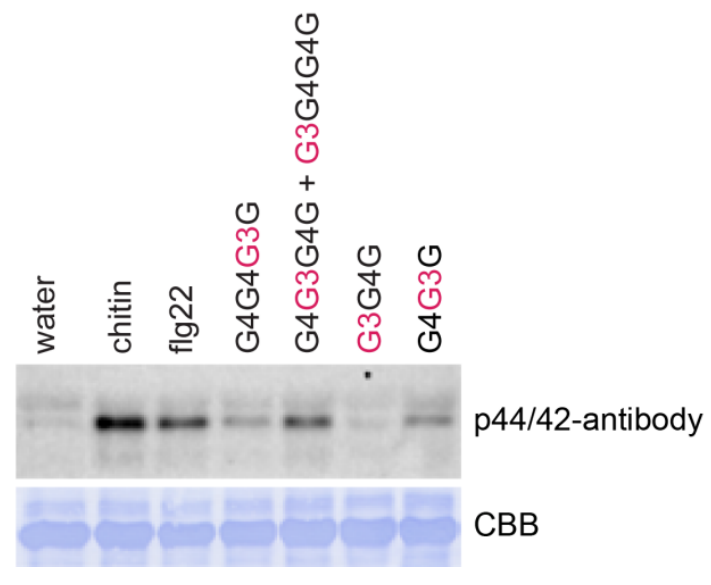

Figure 10. Activation of immune responses upon treatment with MLG oligomers in barley. (A) Generation of ROS in barley upon treatment with MLG oligomers. Leaf discs of second leaves of 10-day old barley plants were treated with water, $100 \mathrm{\mu g} \mathrm{ml}^{-1}$ chitin, $100 \mathrm{nM}$ flg22 or $100 \mathrm{~g} \mathrm{ml}^{-1} \mathrm{MLG}$ oligomers. Data show mean of eight leaf discs and error bars represent SEM. The experiment was performed three times with similar results. (B) MAPK activation upon MLG treatment. Leaf discs of second leaves of barley plants were treated for 12 min with water, $10 \mu \mathrm{g} \mathrm{ml}^{-1}$ chitin, $50 \mathrm{nM} \mathrm{flg22} \mathrm{or} 10 \mu \mathrm{g} \mathrm{ml}^{-1} \mathrm{MLG}$ oligomers. Activation of MAPK was analysed via Western Blot using the p44/42-antibody. Lower panel shows Coomassie Brilliant Blue (CBB) staining as loading control.

\subsubsection{MLGs induce immune responses in Arabidopsis}

In the last years, MLGs were not only shown to be present in the cell wall of monocots, but also in bacteria and fungi. MLGs are present as exopolysaccharides in the endosymbiont Sinorhizobium meliloti (Pérez-Mendoza et al., 2015). Furthermore, MLGs were shown to be abundant cell wall 
components in the two ascomycete fungi Aspergillus fumigatus and Rhynchosporium commune (formerly known as $R$. secalis) (Pettolino et al., 2009; Samar et al., 2015). R. commune is a plant pathogenic fungus causing leaf blotch on barley and the inner hyphal cell walls of $R$. commune were shown to contain MLGs (Pettolino et al., 2009). Due to the presence of MLGs in bacteria and fungi, MLGs might act as a MAMP in plant species that do not contain MLGs, e.g. Arabidopsis.

To test whether MLGs might be able to induce immune responses in Arabidopsis, the four MLGs were analysed regarding their ability to induce the influx of $\mathrm{Ca}^{2+}$ using Arabidopsis seedlings that express the $\mathrm{Ca}^{2+}$ sensor protein aequorin. Also, a luminol-based assay was used to analyse the generation of ROS and an immunoblot analysis was performed to test the phosphorylation of MAPK in Arabidopsis upon MLG treatment. Interestingly, all four MLG oligomers could elicit a rapid influx of $\mathrm{Ca}^{2+}$ in $\mathrm{Col}-0$ with the $\mathrm{Ca}^{2+}$ influx being more intense upon treatment with MLG tetramers compared to treatment with MLG trimers (

Figure $11 \mathrm{~A}$ ). Also, the $\mathrm{Ca}^{2+}$ influx seems to reach its peak faster upon MLG oligomer treatment than upon chitin or flg22 treatment (

Figure $11 \mathrm{~A}$ ). The generation of ROS could only slightly be induced by the tetramer mixture G4G3G4G + G3G4G4G in Col-0, Ws-0 and Ws-4. In Ws-0, the ROS burst was also slight induced by the MLG tetramer G4G4G3G and the MLG trimer G3G4G (

Figure $11 \mathrm{~B}$, Figure $\mathrm{S} 16 \mathrm{~A}$ and $\mathrm{B})$. Furthermore, all four MLG oligomers triggered the activation of MAPKs in Col-0, Ws-0 and Ws-4, in particular of MAPK6 and MAPK3. Notably, MAPK activation was more pronounced upon treatment with MLG tetramers than with MLGs trimers (

Figure $11 \mathrm{C}$, Figure S16 C), suggesting a higher binding affinity of the tetramers to the respective receptor compared to the binding affinity of the trimer to the receptor. Additionally, the level of MAPK activation induced by MLG tetramer treatment was higher compared to the MAPK activation upon treatment with the known carbohydrate DAMP OGs but less pronounced than upon elicitation with chitin or flg22. Although the calcium response, generation of ROS as well as activation of MAPK were stronger upon treatment with chitin or flg22, these results indicate that MLGs have the ability to induce immune responses in Arabidopsis. 


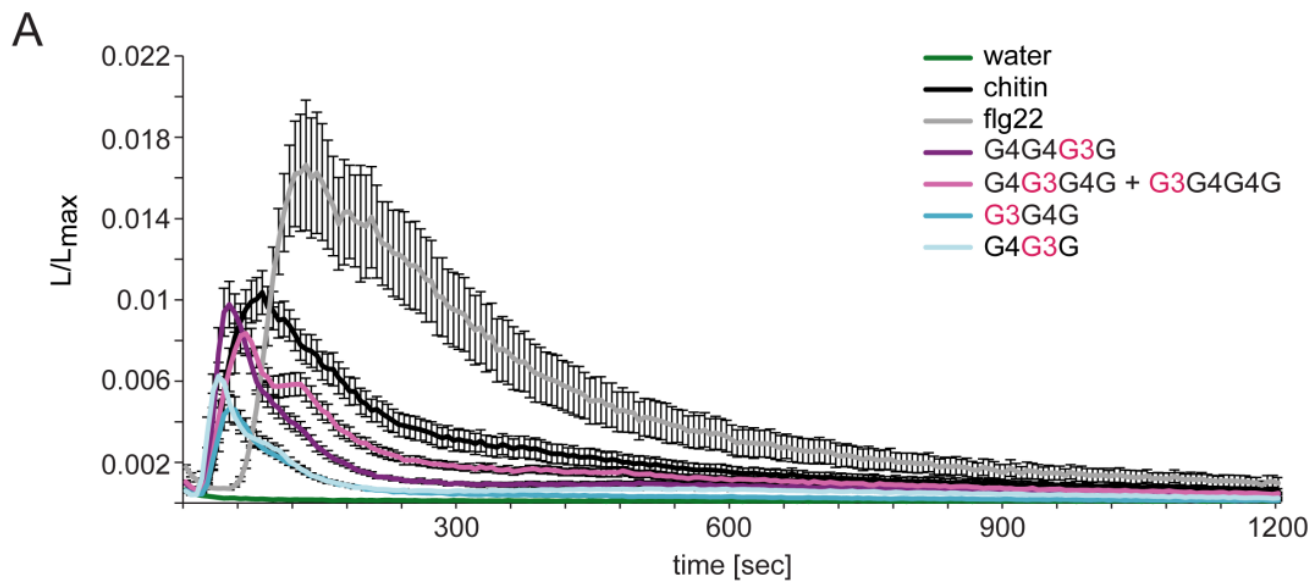

B
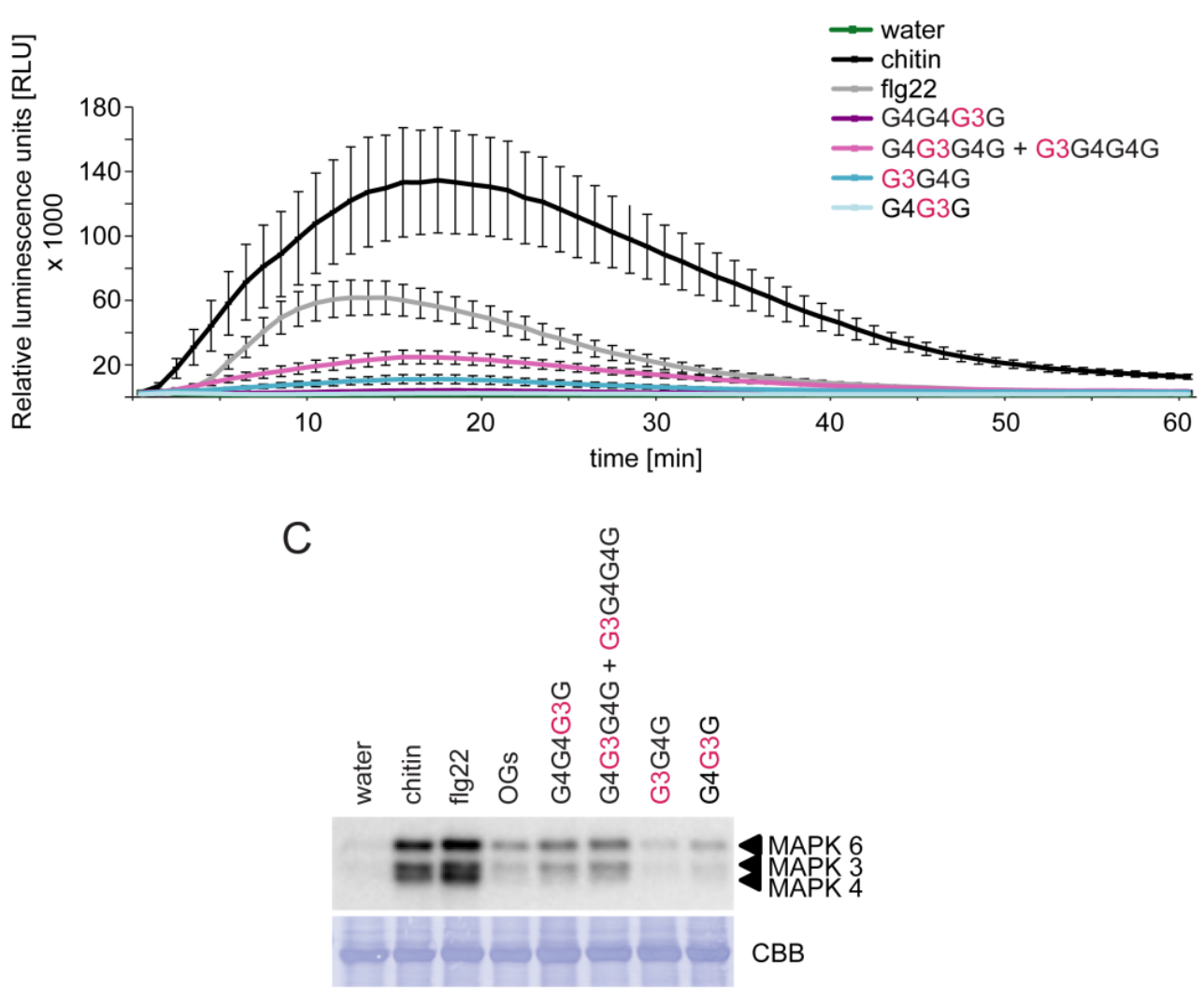

Figure 11. Activation of immune responses in Arabidopsis Col-0 by MLG oligomers from Megazyme. (A) $\mathrm{Ca}^{2+}$ influx upon MLG treatment. 8-10 day old Arabidopsis Col-0 seedlings expressing the $\mathrm{Ca}^{2+}$ sensor protein aequorin were treated with water, $100 \mathrm{\mu g} \mathrm{ml}^{-1}$ chitin, $100 \mathrm{nM}$ flg22 or $100 \mathrm{mg} \mathrm{ml}^{-1} \mathrm{MLG}$ oligomers and the $\mathrm{Ca}^{2+}$ elevation was measured for $1200 \mathrm{sec}$ in $6 \mathrm{sec}$ intervals. To obtain the total remaining luminescence $\left(L_{\max }\right)$, the remaining aequorin was discharged by adding $\mathrm{CaCl}_{2}$ to each well and luminescence was recorded for 3 min in $6 \mathrm{sec}$ intervals. For normalization, the elicitor induced luminescence per $6 \mathrm{sec}(\mathrm{L})$ was divided by $L_{\max }$. The data shown represent the mean of 12 seedlings and error bars represent SEM. The experiment was repeated three times with similar results. (B) Generation of ROS upon MLG treatment. Leaf discs of 5-7-week old Col-0 plants were treated with water, $100 \mathrm{\mu g} \mathrm{ml}^{-1}$ chitin, $100 \mathrm{nM}$ flg22 or different $100 \mathrm{\mu g} \mathrm{ml}^{-1} \mathrm{MLG}$ oligomers. Relative Light Units (RLU) were recorded directly upon the respective treatment for $60 \mathrm{~min}$ in $1 \mathrm{~min}$ intervals. The shown data represent the mean of 8 leaf discs per treatment and error bars represent SEM. The experiment was repeated four times with similar results. (C) MAPK activation upon MLG treatment. 14-day old in-vitro grown Arabidopsis

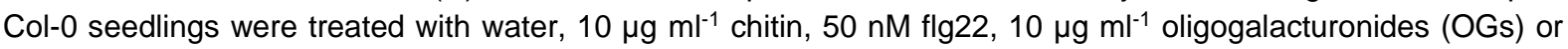
$10 \mu \mathrm{g} \mathrm{ml}^{-1} \mathrm{MLG}$ oligomers for $12 \mathrm{~min}$. Activation of MAPK6, 3 and 4 was analysed by Western Blot using the p44/42 antibody. Lower panel shows Coomassie Brilliant Blue (CBB) staining of the membrane as loading control. The experiment was repeated four times with similar results. 
Besides the influx of $\mathrm{Ca}^{2+}$, generation of ROS and activation of MAPK, activation of PTI also leads to transcriptional reprogramming (Boller and Felix, 2009). To further verify the ability of MLGs to trigger immune responses, the expression of the defence genes WRKY33 and WRKY53 was analysed via qRT-PCR. Upon treatment with the MLG tetramers G4G4G3G and G4G3G4G + G3G4G4G or the MLG trimer G4G3G, the expression of WRKY33 and WRKY53 was induced significantly compared to the water control. The MLG trimer G3G4G did induce the expression of WRKY53 significantly (Figure 12). As observed for the influx of $\mathrm{Ca}^{2+}$ and activation of MAPK, the induction of the expression of WRKY33 and WRKY53 was more pronounced upon treatment with MLG tetramers than with MLG trimers (Figure 12). The ability of MLGs to induce defence gene expression further indicates their potential role as elicitor of immune responses.

A

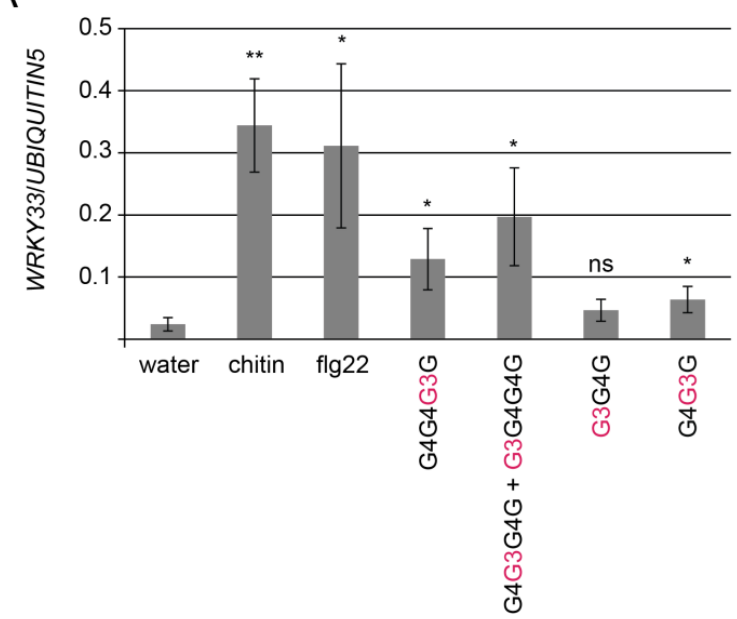

B

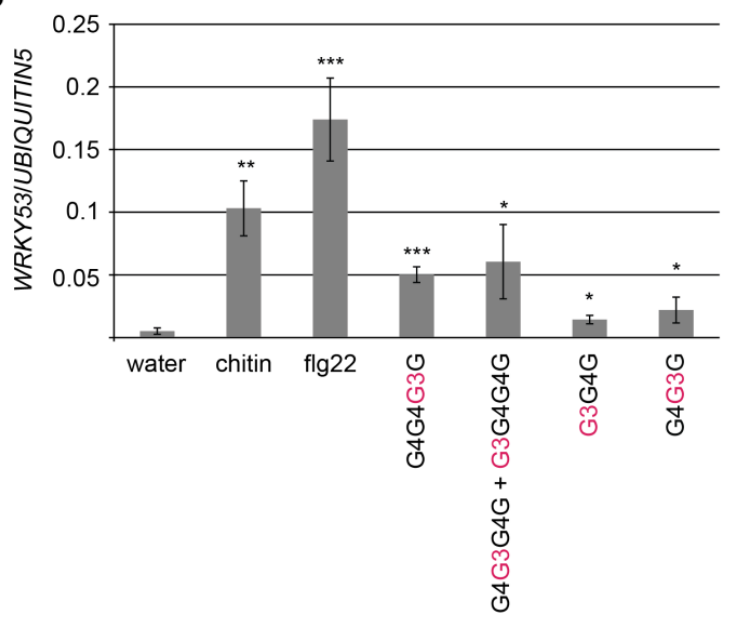

Figure 12. Defence gene expression upon Megazyme MLG oligomer treatment in Arabidopsis Col-0 seedlings. 14-day old in-vitro grown Arabidopsis Col-0 seedlings were treated with water, $10 \mu \mathrm{g} \mathrm{ml}^{-1}$ chitin, $50 \mathrm{nM}$ flg22 or $10 \mu \mathrm{g} \mathrm{ml}^{-1} \mathrm{MLG}$ oligomers for $30 \mathrm{~min}$. Expression of WRKY33 (A) and WRKY53 (B) were analysed using qRT-PCR. UBIQUITIN5 served as reference gene. The bars show the mean of three biological replicates with three technical replicates each. Error bars show standard deviation. Asterisks indicate statistical significance of the elicitor treatments compared to water treatment with not significant $(n s)=p>0.5,{ }^{*}=p \leq 0.5$, ${ }^{* *}=p \leq 0.001$ and ${ }^{* * *}=p \leq 0.001$. The unpaired student's t-teat was used to calculate $p$-values.

\subsubsection{MLG oligosaccharides from a second company can induce immune responses in barley and Arabidopsis}

To verify the ability of MLGs to induce immune responses in barley and Arabidopsis, one MLG tetramer (G4G3G4G) as well as two MLG trimers (G3G4G and G4G3G) were purchased from a second supplier for carbohydrates, Carbosynth (Compton, UK) and tested for their ability to induce immune responses. The two MLG trimers as well as the tetramer G4G4G3G are structurally the same as the MLGs obtained from Megazyme. However, Carbosynth offered the MLG tetramer G4G3G4G 
as a single substance while it was only available as a mixture (G4G3G4G + G3G4G4G) at Megazyme.

Again, the generation of ROS as well as the activation of MAPK upon MLG treatment were analysed in barley. The MLG tetramer G3G4G4G but not the MLG trimers G3G4G and G4G3G did induce the generation of ROS (Figure $13 \mathrm{~A}$ ). Furthermore, the activation of MAPK was clearly induced upon treatment with the tetramer G4G3G4G and comparable to the intensity of MAPK phosphorylation upon flg22. Also, the MLG trimers G3G4G and G4G3G did induce the MAPK activation in barley, however, the level of MAPK activation was lower in comparison to the induction upon MLG tetramer treatment (Figure $13 \mathrm{~B}$ ).
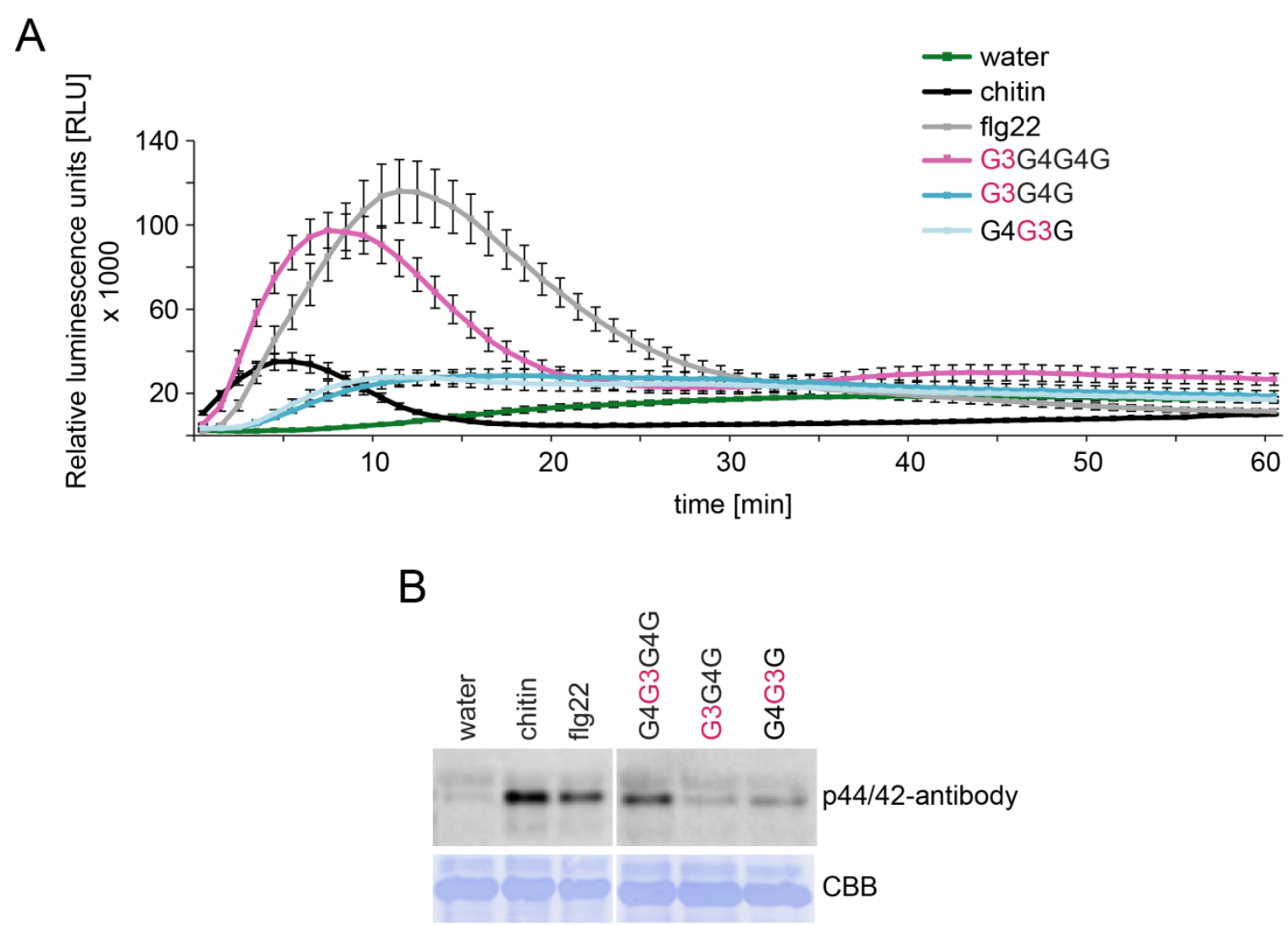

Figure 13. ROS burst generation and activation of MAPK in barley by MLG oligomers from Carbosynth. (A) Generation of ROS in barley upon treatment with a MLG tetramer and two MLG trimers. Leaf discs of second leaves of 10-day old barley plants were treated with water, $100 \mathrm{\mu g} \mathrm{ml}^{-1}$ chitin, $100 \mathrm{nM}$ flg22 or $100 \mu \mathrm{g} \mathrm{ml}^{-1} \mathrm{MLG}$ oligomers. Data show mean of eight leaf discs and error bars represent SEM. The experiment was performed three times with similar results. (B) MAPK activation upon MLG treatment. Leaf discs of second leaves of barley plants were treated for 12 min with water, $10 \mu \mathrm{g} \mathrm{ml}^{-1}$ chitin, $50 \mathrm{nM}$ flg22 or $10 \mu \mathrm{g} \mathrm{ml}^{-1} \mathrm{MLG}$ oligomers. Activation of MAPK was analysed via Western Blot using the p44/42-antibody. Lower panel shows Coomassie Brilliant Blue (CBB) staining as loading control. The experiment was repeated two times with similar results. 
To determine if the three MLGs from Carbosynth are able to induce immune responses in Arabidopsis, Arabidopsis Col-0 seedlings expressing the $\mathrm{Ca}^{2+}$ sensor protein aequorin were used to monitor the calcium response. The tetramer G4G3G4G as well as the two trimers G3G4G and G4G3G induced the influx of $\mathrm{Ca}^{2+}$ ions. The $\mathrm{Ca}^{2+}$ peak induced by the MLG tetramer was stronger in comparison to the $\mathrm{Ca}^{2+}$ influx induced by the two MLG trimers. Also, the $\mathrm{Ca}^{2+}$ influx upon $\mathrm{MLG}$ treatment was faster than upon flg22 treatment (Figure $14 \mathrm{~A}$ ). Next, a luminol-based assay was performed to determine whether MLGs induce the generation of ROS. The ROS burst generation was clearly observed upon treatment with chitin and flg22, however, could not be observed upon treatment with MLG oligomers in Col-0 (Figure 14 B). ROS generation was also not observed in Ws-0 and Ws-4 upon MLG elicitation (Figure S17 A and B). Western blot analysis using the p44/42-antibody revealed that MAPK6 and MAPK3 were phosphorylated upon treatment with the MLG tetramer G4G3G4G and very slightly upon treatment with the MLG trimers G3G4G and G4G3G in Col-0, Ws-0 and Ws-4 (Figure 14 C, Figure S17 C). 
A

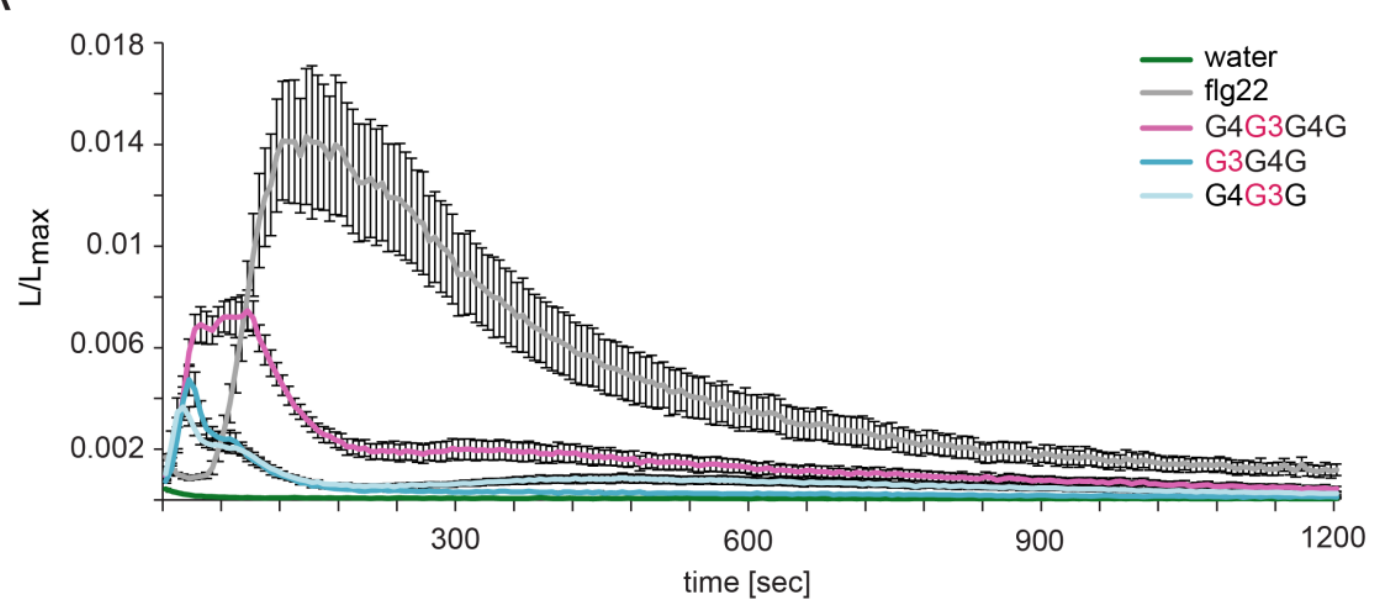

B

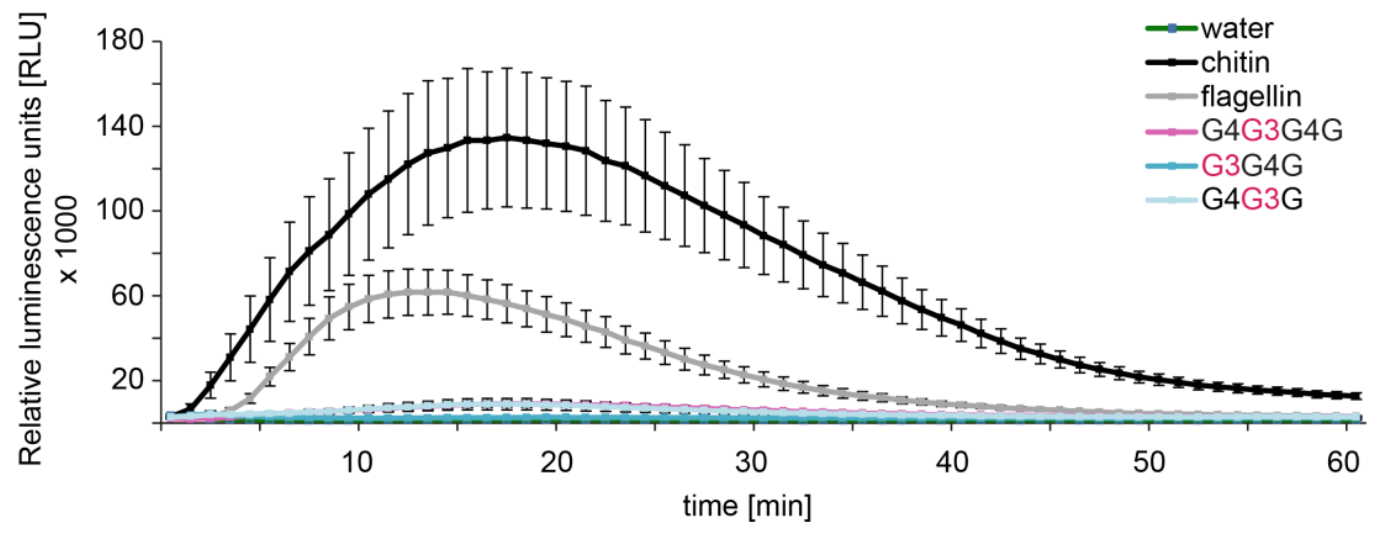

C

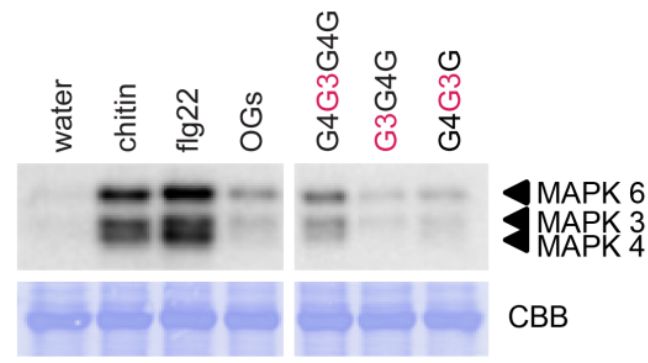

Figure 14. Activation of immune responses in Arabidopsis Col-0 upon treatment with MLG oligomers from Carbosynth. (A) Influx of $\mathrm{Ca}^{2+}$ upon MLG oligomer treatment. 8-10-day old Arabidopsis Col-0 seedlings expressing the $\mathrm{Ca}^{2+}$ sensor protein aequorin were treated with water, $100 \mu \mathrm{g} \mathrm{ml} \mathrm{m}^{-1}$ chitin, $100 \mathrm{nM}$ flg22 or $100 \mathrm{\mu g} \mathrm{ml}^{-1} \mathrm{MLG}$ oligomers. Elevation in $\mathrm{Ca}^{2+}$ was measured in $6 \mathrm{sec}$ intervals for $1200 \mathrm{sec}$. Upon treatment, the total remaining luminescence $\left(\mathrm{L}_{\max }\right)$ was obtained by adding $\mathrm{CaCl}_{2}$ to the wells and luminescence was recorded for $3 \mathrm{~min}$ in $6 \mathrm{sec}$ intervals. For normalization, luminescence upon elicitor treatment per $6 \mathrm{sec}(\mathrm{L})$ was divided by $L_{\text {max. }}$ Data shown represent mean of 12 seedlings with SEM. The experiment was repeated three times with similar results. (B) ROS burst generation upon MLG treatment. Leaf discs of 5-7 week old Arabidopsis Col-0 plants were treated with water, $100 \mathrm{\mu g} \mathrm{ml}^{-1}$ chitin, $100 \mathrm{nM}$ flg22 or $100 \mathrm{\mu g} \mathrm{ml}^{-1} \mathrm{MLG}$ oligomers and relative light units (RLU) were recorded every minute for $60 \mathrm{~min}$. The shown data represent mean of 8 leaf discs with SEM. The experiment was repeated two times with similar results. (C) Activation of MAPK upon MLG treatment. In-vitro grown 14-day old Col-0 seedlings were treated with water, $10 \mu \mathrm{g} \mathrm{ml}^{-1}$ chitin, $50 \mathrm{nM}$ flg22 or $10 \mu \mathrm{g} \mathrm{ml}^{-1} \mathrm{MLG}$ oligomers for $12 \mathrm{~min}$. Activation of MAPK6, MAPK3 and MAPK4 was analysed via Western Blot using the p-44/42 antibody. The lower panel shows Coomassie Brilliant Blue (CBB) staining as loading control. The experiment was repeated three times with similar results. 
Additionally, it was tested whether the three MLGs from Carbosynth are able to induce defence gene expression. Thus, the expression of WRKY33 and WRKY53 upon MLG elicitation was analysed via qRT-PCR. The tetramer G4G3G4G induced the transcriptional upregulation of WRKY33 and WRKY53 10-fold and 12-fold, respectively, compared to water (Figure 15). The trimer G3G4G did significantly induce the transcription of WRKY53 (Figure 15). As observed before, the induction of defence gene expression was more pronounced upon treatment with the MLG tetramer than with the MLG trimers.

All ordered MLGs from the second company Carbosynth did induce immune responses in barley as well as in Arabidopsis indicating that MLGs display elicitor activities.

A

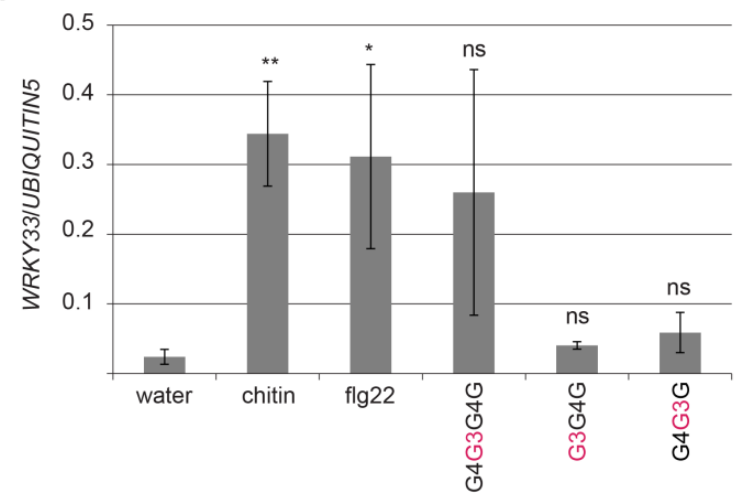

B

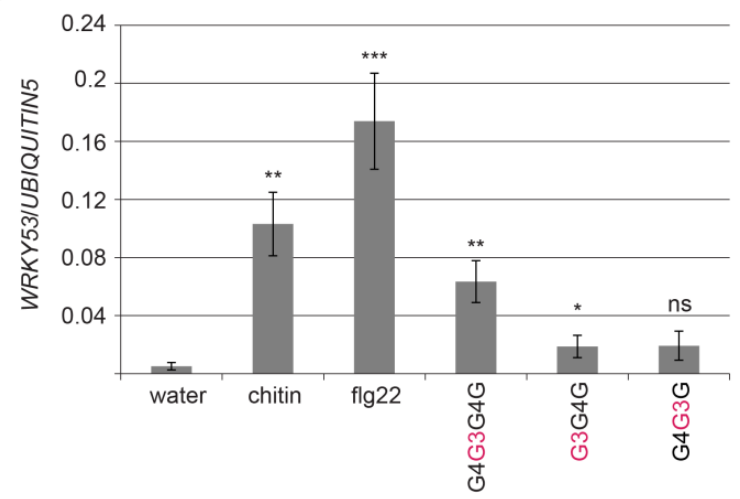

Figure 15. Expression of defence genes upon treatment with MLG oligomers from Carbosynth. In-vitro grown 14-day old Arabidopsis Col-0 seedlings were treated with water, $10 \mu \mathrm{g} \mathrm{ml}{ }^{-1}$ chitin, $50 \mathrm{nM}$ flg22 or $10 \mathrm{\mu g} \mathrm{ml}^{-1} \mathrm{MLG}$ oligomers for $30 \mathrm{~min}$. Expression of the genes WRKY33 (A) and WRKY53 (B) were tested using qRT-PCR. UBIQUITIN5 served as reference gene. The bars show the mean of three biological replicates consisting of three technical replicates each and error bar represents standard deviation. Statistical significance of the elicitor treatments compared to water treatment is indicated by asterisks with not significant (ns) $=p>0.5$, ${ }^{*}=p \leq 0.5,{ }^{* *}=p \leq 0.001$ and ${ }^{* * *}=p \leq 0.001$. The unpaired student's t-teat was used to calculate $p$-values.

\subsubsection{Commercially available MLGs do not contain major quantitative contaminants}

To exclude the possibility that a potential contamination in the MLG preparations from Megazyme (Bray, Ireland) and Carbosynth (Compton, UK) induce the immune responses in Arabidopsis and barley, all MLG tetramers and trimers were tested for purity via HPAEC-PAD and matrix-assisted laser desorption ionization with time-of-flight detection (MALDI-TOF).

First, the commercially available MLG tetramers and trimers were analysed via HPAEC-PAD for potential carbohydrate contaminations. No major contaminations could be found in the HPAEC-PAD analysis of the MLG tetramer G4G4G3G from Megazyme, the MLG tetramer mixture G4G3G4G + G3G4G4G from Megazyme and both MLG trimers, G3G4G and G4G3G, from Megazyme and Carbosynth. Furthermore, the respective MLG tetramer and trimers from Megazyme and Carbosynth show the same retention times indicating that they have the same MLG structure (Figure $16 \mathrm{~A}, \mathrm{C}$ and D). Unexpectedly, the MLG tetramer G4G3G4G from Carbosynth did show two peaks in the 
HPAEC-PAD analysis indicating that two carbohydrates are present in this preparation (Figure $16 \mathrm{~B}$ ). The HPAEC-PAD profile of G4G3G4G from Carbosynth is similar to the HPAEC-PAD profile obtained for the MLG tetramer mixture G4G3G4G + G3G4G4G from Megazyme suggesting that the MLG tetramer G4G3G4G from Carbosynth is not a single MLG tetramer but is also a mixture of G4G3G4G + G3G4G4G (Figure 16 B). Furthermore, minor peaks were observed in the HPAEC-PAD chromatograms of all MLG preparations (Figure 16). Since the MLG tetramers and trimers were generated by hydrolyzing the barley $\beta-1,3 ; 1-4$-glucan polymer, these peaks likely correspond to carbohydrates containing less than four glucose monomers e.g. cellotriose, cellobiose or laminaribiose. However, the low intensity of these peaks suggests that their abundance in the preparation is very low and consequently, is negligible.

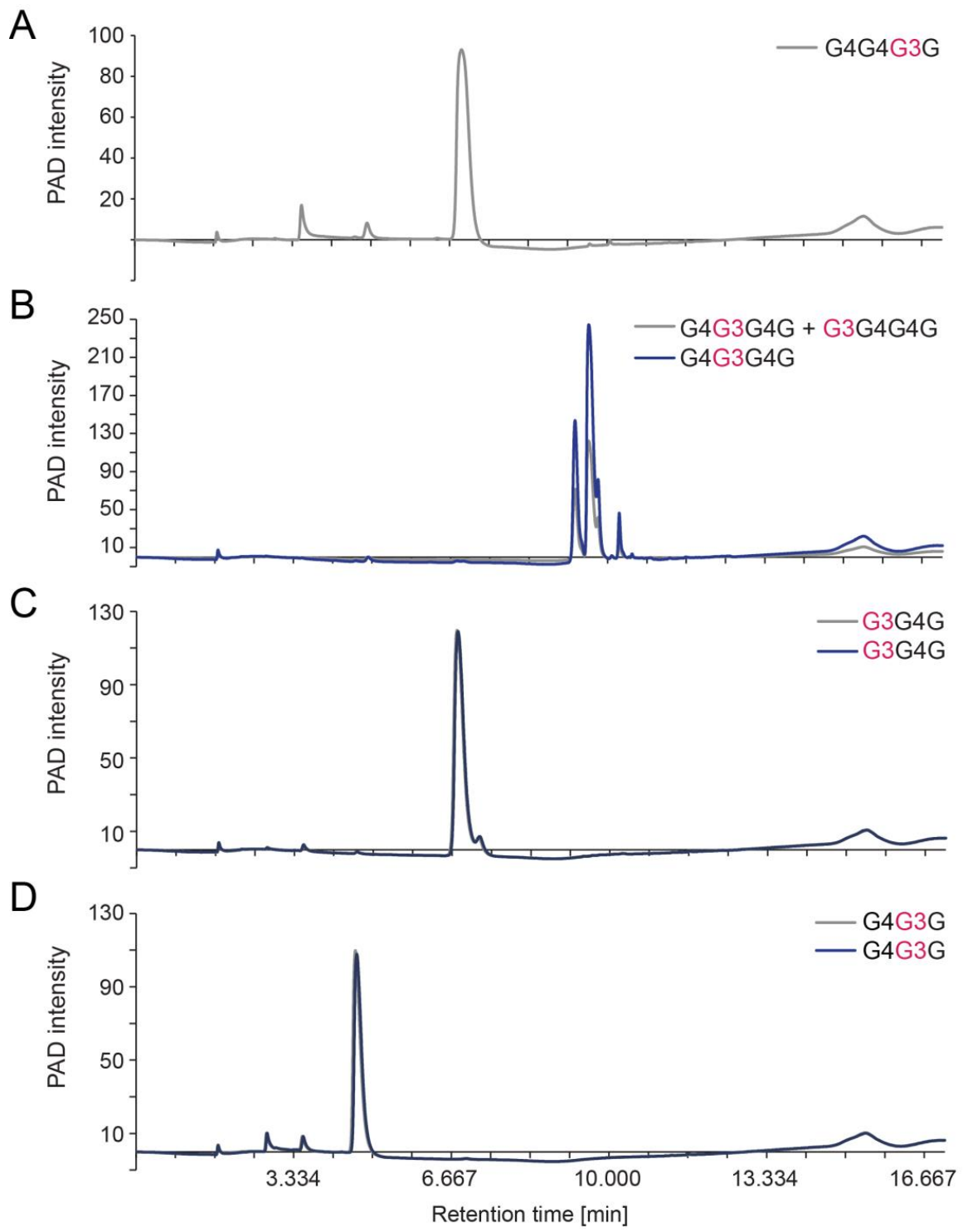

Figure 16. HPAEC-PAD chromatographs of MLG oligomers. (A) to (D) HPAEC-PAD profiles of MLG oligomers from Megazyme (grey) and (B) to (D) HPEAC-PAD chromatograms of MLG oligomers from Carbosynth (blue). $56 \mu \mathrm{M}$ of the respective MLG tetramers and $45 \mu \mathrm{M}$ of the respective MLG trimers were analysed via HPAEC-PAD and checked for carbohydrate contamination. 
MALDI-TOF can be used to determine the molecular weight of a compound. Therefore, the sample for analysis is mixed with a matrix on a MALDI plate. The matrix as well as the sample are allowed to dry and crystallize. Upon drying, a laser beam is applied to the matrix-sample mixture and the matrix-sample mixture is ionized. The generated ions from the sample are accelerated at a fixed potential and separated according to their mass-to-charge ratio $(\mathrm{m} / \mathrm{z})$ (Hosseini and Martinez-Chapa, 2017). To verify the masses of all MLG tetramers and MLG trimers and to exclude possible contaminations, a MALDI-TOF analysis for all MLG tetramers and trimers was performed. For the MLG tetramers a signal $666.6 \mathrm{~m} / \mathrm{z}$ and for the MLG trimers a signal at $504.4 \mathrm{~m} / \mathrm{z}$ was expected. During the MALDI-TOF analysis of the MLG tetramers, both $\mathrm{Na}^{+}$adducts $(666.6+22.9 \mathrm{~m} / \mathrm{z})$ and $\mathrm{K}^{+}$adducts $(666.6+39 \mathrm{~m} / \mathrm{z})$ were observed (Figure $\left.17 \mathrm{~A}-\mathrm{C}\right)$. Also, in the MALDI-TOF analysis of the MLG trimers $\mathrm{Na}^{+}(504.4+22.9 \mathrm{~m} / \mathrm{z})$ as well as $\mathrm{K}^{+}$adducts $(504.4+39 \mathrm{~m} / \mathrm{z})$ were found (Figure $17 \mathrm{D}$ $H)$. These results together with the HPAEC-PAD analysis indicate pure MLG preparations and support the companies' claim of high purity. 


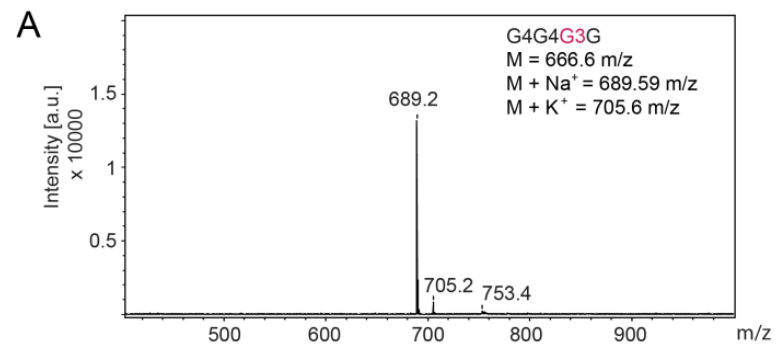

B
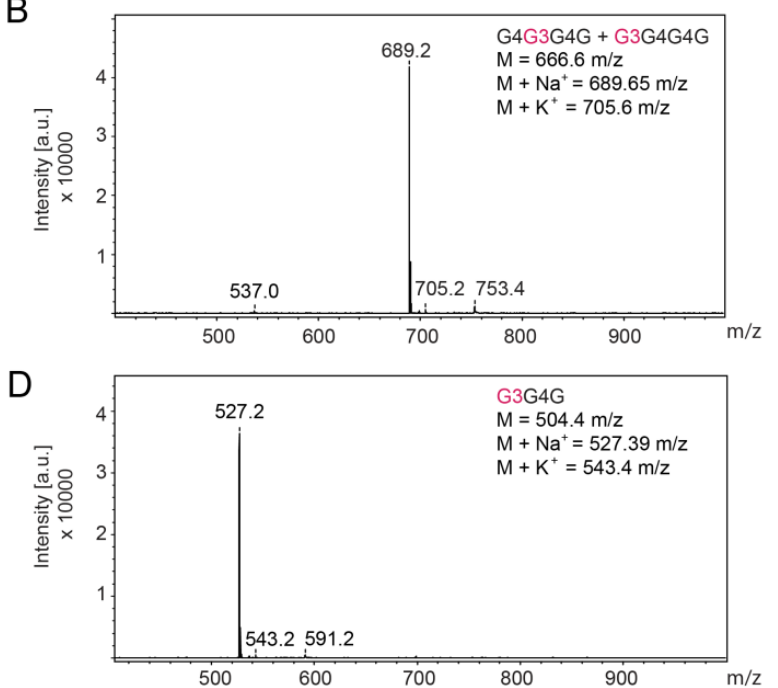

F

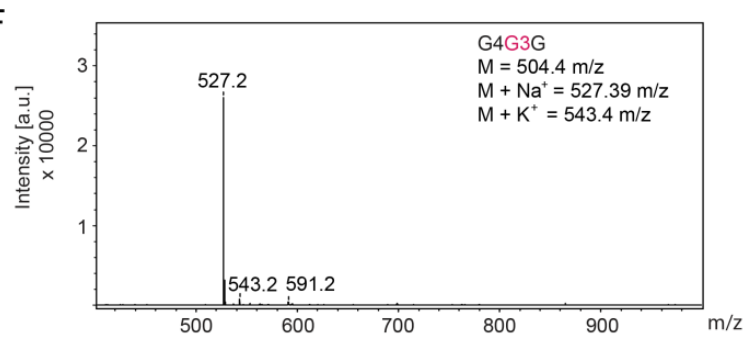

C

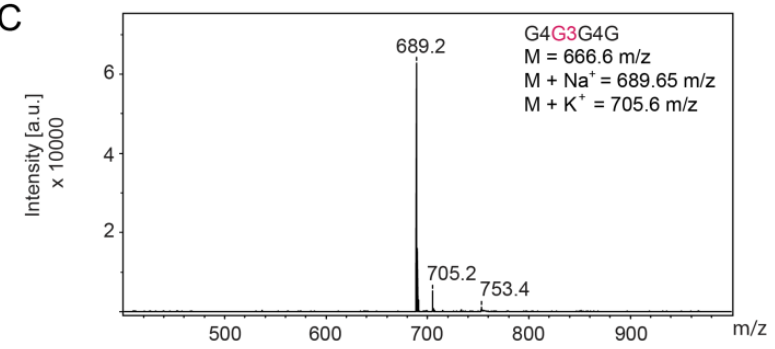

$\mathrm{E}$

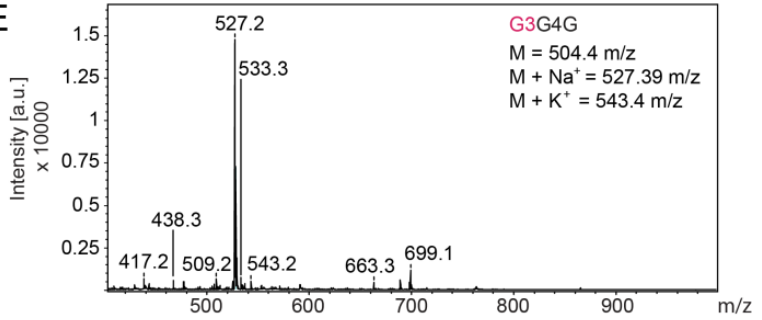

G

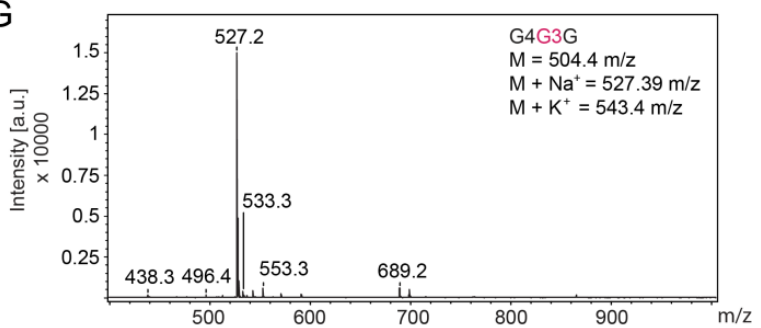

Figure 17. Verification of masses of MLG oligomers. $10 \mathrm{\mu g} \mathrm{ml}^{-1}$ of the respective MLG oligomers from Megazyme (A, B, D, F) or Carbosynth (C, E, G) were mixed with a 2,5-Dihydrobenzoic acid MALDI MATRIX. The masses of the oligomers was analysed using MALDI-TOF. Expected masses of the pure MLG tetramers and trimers as well as the $\mathrm{Na}^{+}$and $\mathrm{K}^{+}$adducts are indicated.

\subsubsection{Hydrolysis products of the barley $\beta-1,3 ; 1,4$-glucan polymer induce defence responses in Arabidopsis}

The commercially available MLG tetramers and MLG trimers are derived from $\beta-1,3 ; 1,4$-glucan polymers that were hydrolysed with enzymes that cleave $\beta-1,3 ; 1,4$-glucan polymers (Megazyme). Enzymes that act on $\beta-1,3 ; 1,4$-glucan polymers exhibit different specificities regarding the position of the cleaved linkage. An endo- $\beta-1,4-$ glucanase from Aspergillus japonicus hydrolyses $\beta$-1,4-linkages preceding a $\beta$-1,3-linkage resulting in MLG oligosaccharides with the structure $G 3 G 4_{n} G$ with $n$ describing the number of glucose units (Grishutin et al., 2006). However, the commercially available Bacillus subtilis endo-1,3:1,4- $\beta$-D-glucanase (lichenase) cleaves $\beta$-1,4-linkages adjacent to $\beta-1,3$-linkages and consequently, generates MLG oligosaccharides with the general structure 
G4nG3G (Figure 18) (Planas, 2000). Full lichenase hydrolysis of $\beta-1,3 ; 1,4$-polymers of grasses and cereals with the $B$. subtilis lichenase result mainly in the generation of the MLG tetramer G4G4G3G and the trimer G4G3G. However, intermediate products can be achieved upon a partial digest. These intermediate products can e.g. be a hexasaccharide with the structure G4G3G4G4G3G that eventually would by cleaved into two trimers (Figure 18) (Fry et al., 2008). Thus, the B. subtilis lichenase can be used to generate MLG oligosaccharides of varying length but the general structure G4nG3G.

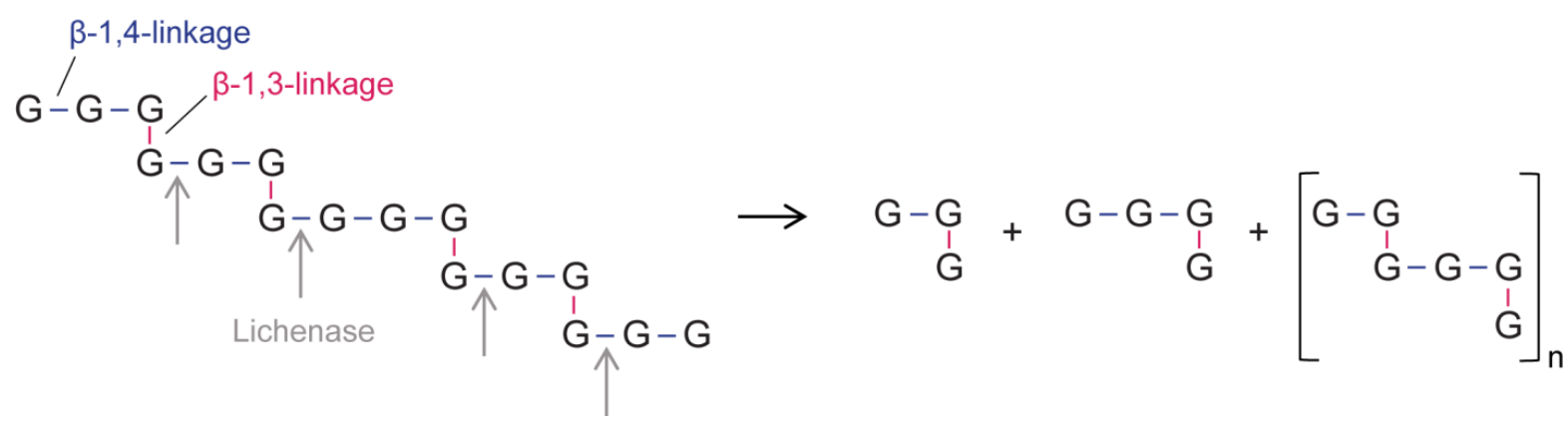

Figure 18. Schematic representation of a $\beta-1,3 ; 1,4$-glucan polymer and the obtained oligosaccharides upon treatment with $\boldsymbol{B}$. subtilis lichenase. Abbreviations: $\mathrm{G}$ - glucose, $\mathrm{n}$-number of residues linked together.

To further verify the ability of MLG oligosaccharides to elicit immune responses, MLG oligosaccharides of varying length were generated using the barley $\beta-1,3 ; 1,4$-glucan polymer and the $B$. subtilis lichenase. In a next step, the obtained MLG oligosaccharides were analysed regarding their capacity to induce PTI responses including the calcium response, activation of MAPK and induction of defence gene expression.

First, optimal conditions for barley $\beta-1,3 ; 1,4-$ glucan polymer hydrolysis were identified by using different buffers with varying $\mathrm{pHs}$ and different enzyme concentrations. Samples were taken upon different incubation times and consequently, the lichenase was inactivated by incubating the hydrolysate for $15 \mathrm{~min}$ in boiling water. The hydrolysis was checked via Thin Layer Chromatography (TLC). The goal was to obtain hydrolysates in which the MLG oligosaccharide concentration increases over time. Optimal conditions were achieved by hydrolyzing the barley $\beta-1,3 ; 1,4$-glucan polymer in $100 \mathrm{mM}$ Sodium phosphate buffer $(\mathrm{pH}=6.5)$ at $40^{\circ} \mathrm{C}$ with either $0.025 \mathrm{U} \mathrm{ml}^{-1}$ or $0.05 \mathrm{U} \mathrm{ml}^{-1}$ lichenase and taking samples upon incubation for 0, 5, 15, 30, 45, 60, 120 and $240 \mathrm{~min}$ (Figure S18). No MLG oligosaccharides can be found in the sample taken upon 0 min incubation time while the amount of MLG oligosaccharides increases over time. The MLG oligosaccharides obtained upon 45 min or longer incubation times co-migrated with the standards for G4G4G3G and G4G3G indicating that the tetramer as well as the trimer are abundant in the respective hydrolysates. Additionally, MLG oligosaccharides were obtained that migrated more slowly than the MLG tetramer and the MLG trimer suggesting that these oligosaccharides are longer than the trimer and tetramer. However, the exact length cannot be determined from the TLC since MLG standards with a length of 
six or more glucose monomers are not commercially available. The $\beta-1,3 ; 1,4-$ glucan polymer can still be found in the hydrolysates, however, the hydrolysates were not further processed to obtain a polysaccharide-free MLG oligosaccharide mixture (Figure S18).

Again, to determine whether the enzymatically generated MLG oligosaccharides have the ability to induce the influx of $\mathrm{Ca}^{2+}$ ions, the calcium responses was monitored in Arabidopsis Col-0 seedlings expressing the $\mathrm{Ca}^{2+}$ sensor protein aequorin. Upon treatment with MLG oligosaccharides that were obtained upon incubation of the $\beta-1,3 ; 1,4$-glucan polymer with either $0.05 \mathrm{U} \mathrm{ml}^{-1}$ or $0.025 \mathrm{U} \mathrm{ml}^{-1}$ active lichenase for 30,60 or 240 min, a rapid increase in $\mathrm{Ca}^{2+}$ was observed. Comparing the intensities of the induced $\mathrm{Ca}^{2+}$ influx upon treatment with MLG oligosaccharides upon the three different incubation times, the MLG oligosaccharides obtained upon 60 min incubation induced the lowest $\mathrm{Ca}^{2+}$ influx while the strongest $\mathrm{Ca}^{2+}$ peak was observed with MLG oligosaccharides obtained upon incubation for $240 \mathrm{~min}$. This suggests a positive correlation between the MLG oligosaccharide concentration and intensity of $\mathrm{Ca}^{2+}$ influx (Figure $19 \mathrm{~A}$ and $\mathrm{B}$ ). In comparison to the $\mathrm{Ca}^{2+}$ peak upon chitin treatment, the $\mathrm{Ca}^{2+}$ spikes resulting from MLG oligosaccharide treatment occurred faster. $\mathrm{No} \mathrm{Ca}^{2+}$ influx was induced upon control treatments with either Sodium phosphate buffer, the untreated $\beta-1,3 ; 1,4$-glucan polymer or the $\beta-1,3 ; 1,4$-glucan polymer incubated with heat-inactivated lichenase $\left(0.05 \mathrm{U} \mathrm{ml}^{-1}\right.$ or $0.025 \mathrm{U} \mathrm{ml}^{-1}$ ) indicating that only MLG oligosaccharides that were enzymatically produced from a MLG polymer can induce a $\mathrm{Ca}^{2+}$ influx (Figure $19 \mathrm{~A}$ and $\mathrm{B}$ ). 

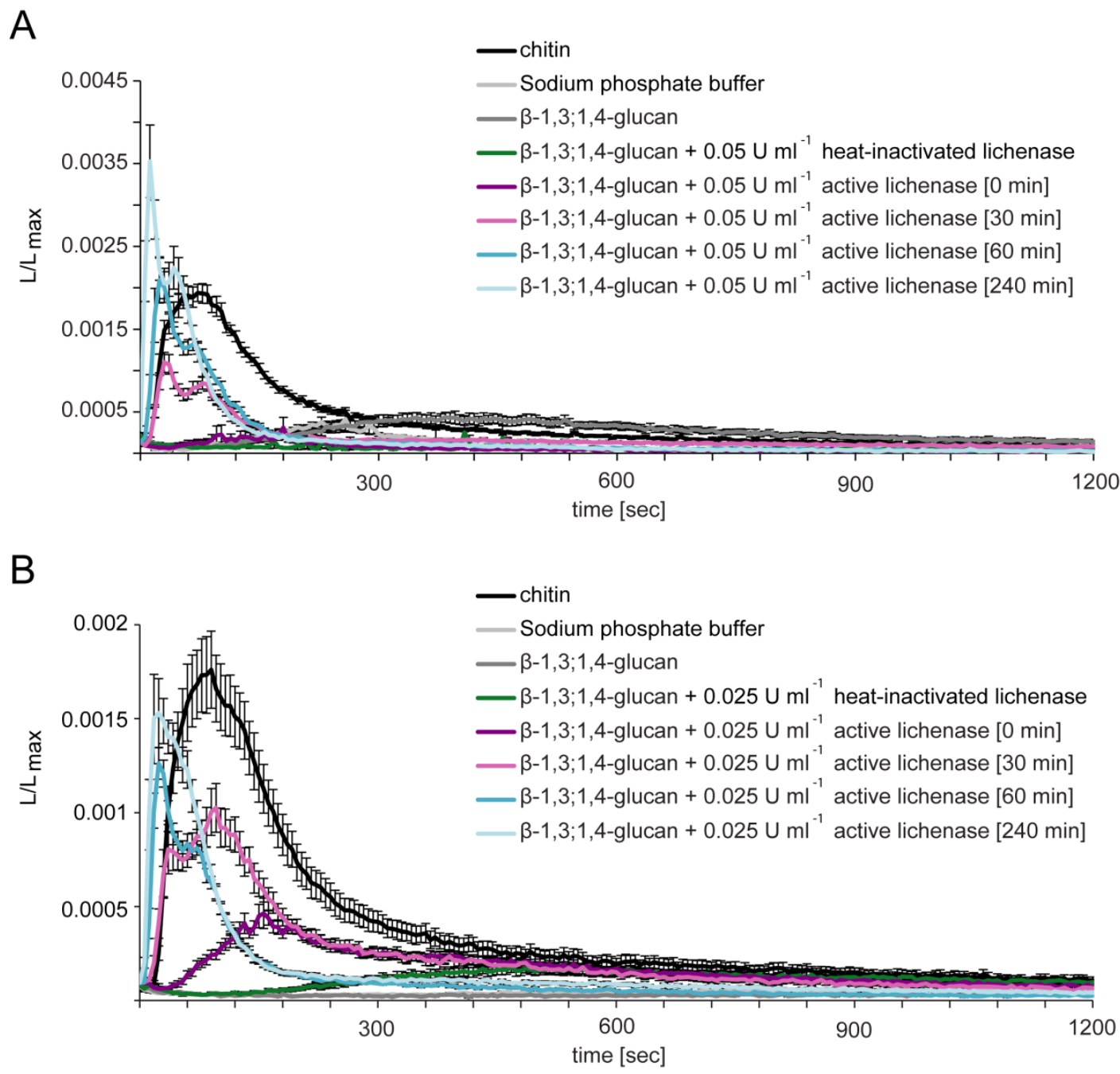

Figure 19. Calcium influx in Arabidopsis Col-0 upon treatment with MLG oligosaccharides. To obtain MLG oligosaccharides of varying length, $10 \mathrm{mg} \mathrm{ml}^{-1}$ barley $\beta-1,3 ; 1,4-$ glucan polymer dissolved in $100 \mathrm{mM}$ Sodium Phosphate buffer $(\mathrm{pH}=6.5)$ was hydrolysed with $0.05(\mathrm{~A})$ or 0.025 (B) $\mathrm{U} \mathrm{ml}^{-1}$ lichenase and the reaction was stopped upon $0,5,15,30,45,60,120$ or 240 min incubation. 8-10 day old Arabidopsis Col-0 seedlings expressing the $\mathrm{Ca}^{2+}$ sensor protein aequorin were treated with $10 \mu \mathrm{g} \mathrm{ml}^{-1}$ chitin, $10 \mathrm{mM}$ Sodium Phosphate buffer, $1 \mathrm{mg} \mathrm{ml}^{-1} \beta-1,3 ; 1,4$-glucan, 0.05 or $0.025 \mathrm{U} \mathrm{ml}^{-1}$ heat inactivated lichenase, $1 \mathrm{mg} \mathrm{ml}^{-1}$ $\beta-1,3 ; 1,4$-glucan plus either 0.05 or $0.025 \mathrm{U} \mathrm{ml}^{-1}$ heat-treated lichenase or a $1: 10$ dilution of MLG oligosaccharides ( $\beta-1,3 ; 1,4$-glucan +0.05 or $0.025 \mathrm{U} \mathrm{ml}^{-1}$ active lichenase incubated for $0,30,60$ or $240 \mathrm{~min}$ ). The $\mathrm{Ca}^{2+}$ elevation upon treatment $(\mathrm{L})$ was recorded in $6 \mathrm{sec}$ intervals for $1200 \mathrm{sec}$. To obtain the total remaining luminescence ( $\left.\mathrm{Lmax}_{\mathrm{m}}\right), \mathrm{CaCl}_{2}$ was added to the wells and luminescence was recorded for $3 \mathrm{~min}$ in $6 \mathrm{sec}$ intervals. For normalization, luminescence counts per $6 \mathrm{sec}$ upon treatment $(L)$ were divided by $L_{\text {max. }}$ Data shown represent mean of 12 seedlings with SEM. The experiment was performed twice with similar results.

Furthermore, the MAPK activation upon MLG oligosaccharide elicitation was tested via immunoblot analysis using the p44/42-antibody. Treatment with active lichenase did not induce MAPK activation in Arabidopsis Col-0 suggesting that Col-0 does not contain MLGs itself which could be cleaved upon pathogen attack and consequently, lead to the activation of immune responses. The activity of the lichenase was verified by testing the hydrolysis of barley $\beta-1,3 ; 1,4$-glucan polymer in $1 / 2$ MS plus sucrose medium and the hydrolysis was analysed via TLC (Figure S19). Additionally, the control treatments as well as the $\beta-1,3 ; 1,4$-glucan polymer incubated with either $0.05 \mathrm{U} \mathrm{ml}^{-1}$ or $0.025 \mathrm{U} \mathrm{ml}^{-1}$ 
lichenase for 0 min did not induce MAPK activation. MAPK6 and MAPK3 phosphorylation was only induced upon treatment with MLG oligosaccharides obtained from $\beta-1,3 ; 1,4$-glucan polymer incubated with either $0.05 \mathrm{U} \mathrm{ml}^{-1}$ or $0.025 \mathrm{U} \mathrm{ml}^{-1}$ lichenase for at least $15 \mathrm{~min}$ (Figure 20). The intensity of the MAPK activation increased over time, thus correlating with increasing amounts of MLG oligosaccharides. This result demonstrates that oligomeric MLGs act as elicitor of immune responses (Figure 20).

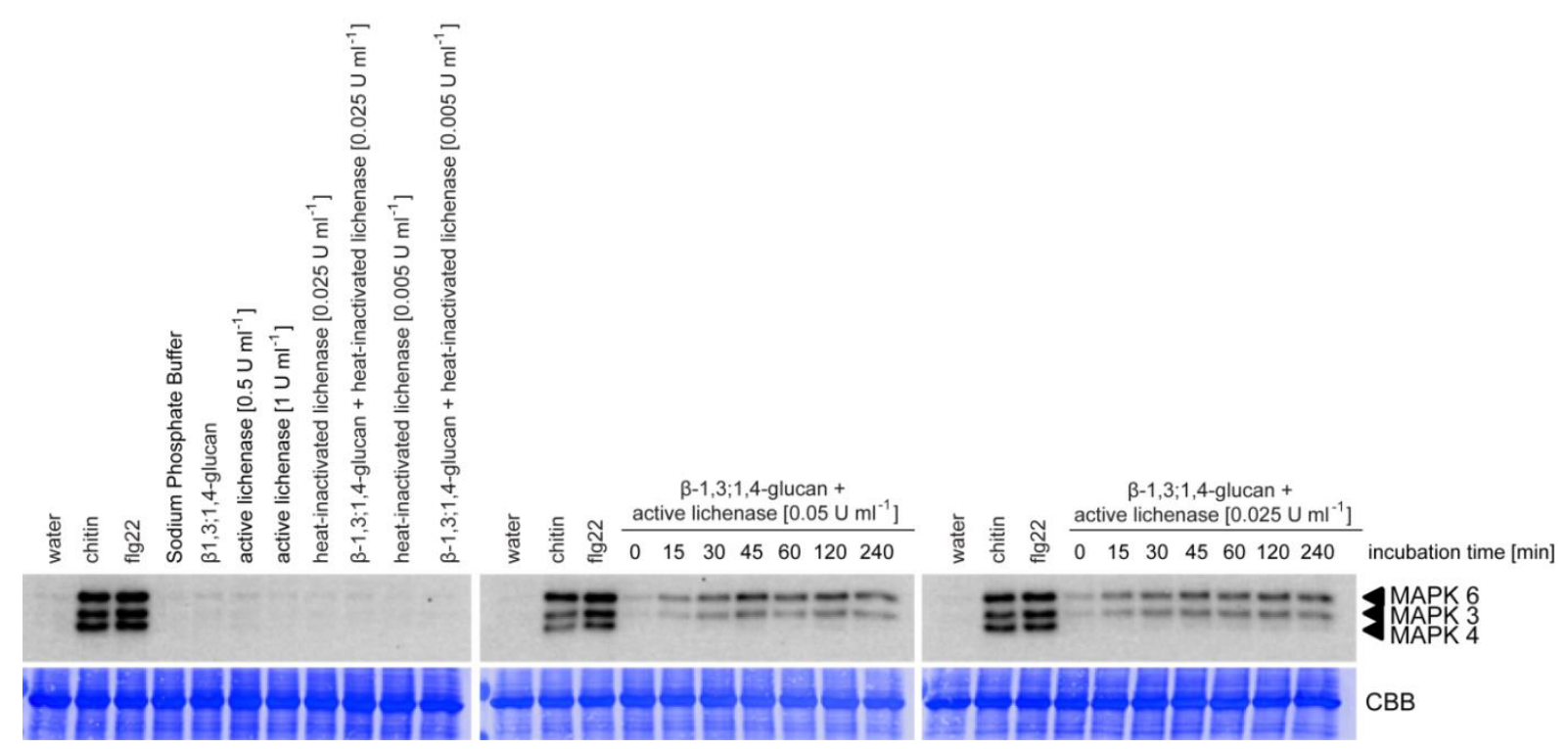

Figure 20. Activation of MAPK in Arabidopsis Col-0 upon treatment with MLG oligosaccharides. To obtain MLG oligosaccharides of varying length, $10 \mathrm{mg} \mathrm{ml}^{-1}$ barley $\beta-1,3 ; 1,4$-glucan polymer dissolved in $100 \mathrm{mM}$ Sodium Phosphate buffer was hydrolysed with 0.05 or $0.025 \mathrm{U} \mathrm{ml}^{-1}$ lichenase and the reaction was stopped upon $0,5,15,30,45,60,120$ or 240 min incubation. In-vitro grown 14-day old Arabidopsis Col-0 seedlings were treated for $12 \mathrm{~min}$ with $10 \mu \mathrm{g} \mathrm{mL} \mathrm{m}^{-1}$ chitin, $50 \mathrm{nM} \mathrm{flg22,} 10 \mathrm{mM}$ Sodium Phosphate buffer, $1 \mathrm{mg} \mathrm{ml}^{-1}$ $\beta-1,3 ; 1,4$-glucan, 0.005 or $0.0025 \mathrm{U} \mathrm{ml}^{-1}$ heat inactivated lichenase, $1 \mathrm{mg} \mathrm{ml}^{-1} \beta-1,3 ; 1,4$-glucan with 0.0025 or $0.005 \mathrm{U} \mathrm{ml}^{-1}$ heat-treated lichenase or a 1:10 dilution of MLG oligosaccharides $(\beta-1,3 ; 1,4$-glucan + active lichenase upon different incubation times). Activation of MAPK6, MAPK3 and MAPK4 was analysed via Western Blot with the p44/42-antibody. Lower panel shows Coomassie Brilliant Blue (CBB) staining as loading control. The experiment was performed two times with similar results.

To further investigate whether the enzymatically generated MLG oligosaccharides trigger immune responses in Arabidopsis, the expression of the defence genes WRKY33 and WRKY53 was tested via qRT-PCR. The expression of the defence gene WRKY33 is significantly induced upon treatment with MLG oligosaccharides upon hydrolysis of $\beta-1,3 ; 1,4$-glucan polymer for all different incubation times (Figure $21 \mathrm{~A}$ and $\mathrm{C}$ ). Similarly, WRKY53 expression was induced significantly upon treatment with MLG oligosaccharides obtained from $\beta$-1,3;1,4-glucan polymer incubated with $0.05 \mathrm{U} \mathrm{ml}^{-1}$ lichenase for 15, 30, 120 and 240 min or MLG oligosaccharides obtained from $\beta$-1,3;1,4-glucan polymer incubated with $0.025 \mathrm{U} \mathrm{ml}^{-1}$ lichenase for 15, 30, 45, 60 and $240 \mathrm{~min}$ (Figure $21 \mathrm{~B}$ and D). As the expression of the two tested defence genes was only significantly induced upon treatment with MLG oligosaccharides but not upon control treatments including Sodium phosphate buffer, this result 
confirms that oligomeric MLGs are perceived by Arabidopsis which subsequently leads to the initiation of defence responses.

A

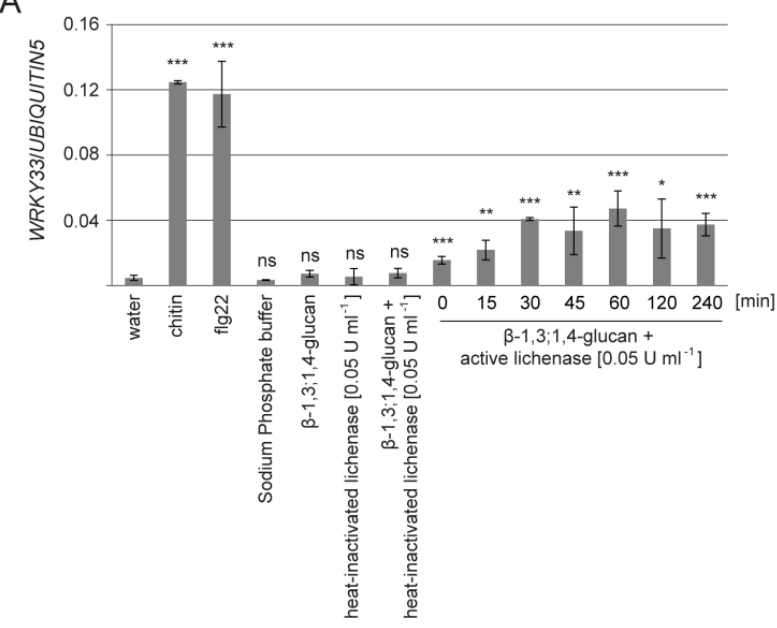

C

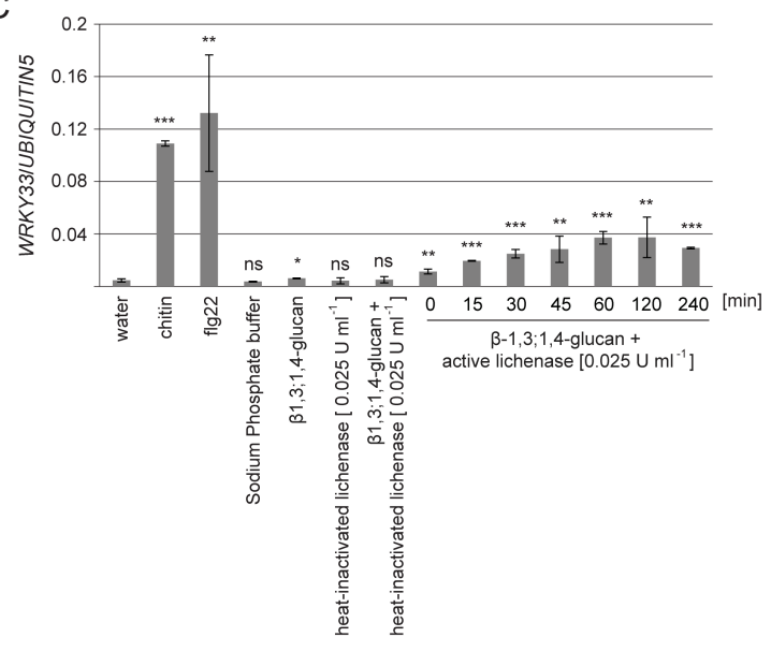

B

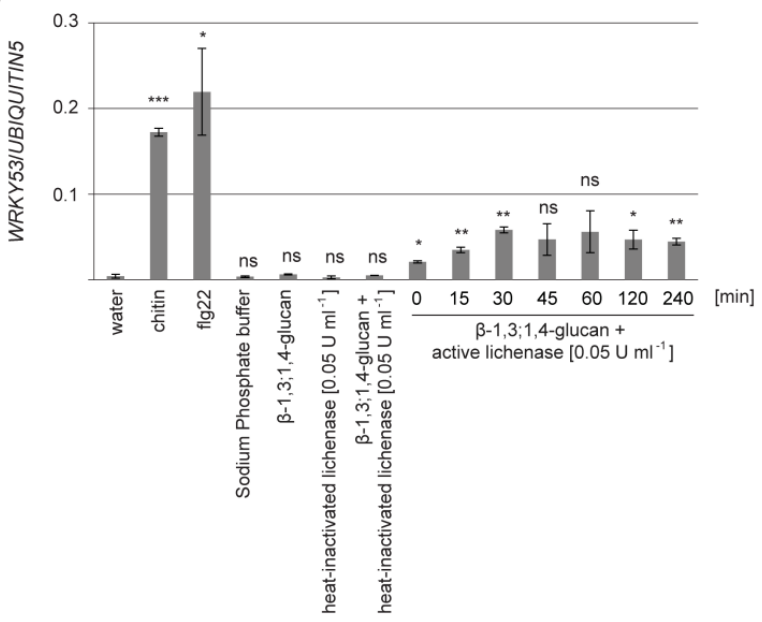

D

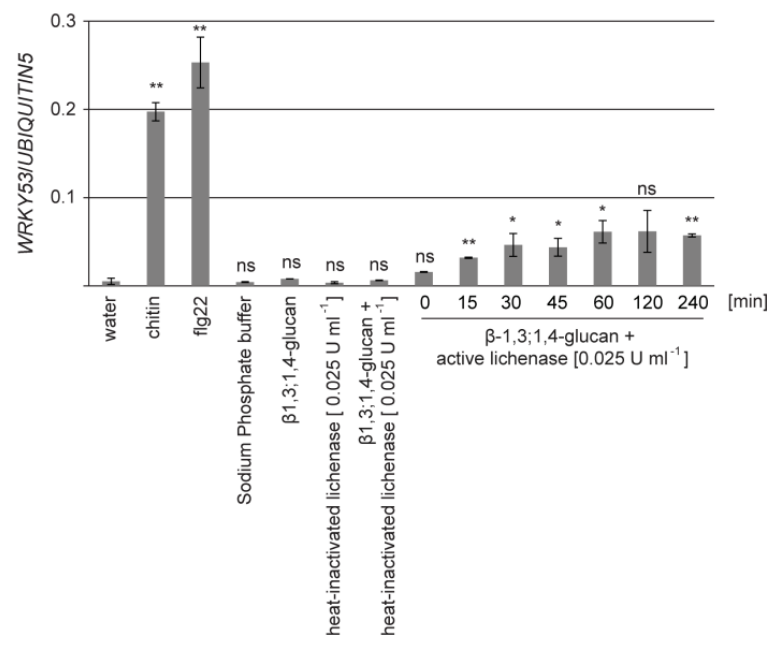

Figure 21. Defence gene expression in Arabidopsis Col-0 upon treatment with MLG oligosaccharides. To obtain MLG oligosaccharides of varying length, $10 \mathrm{mg} \mathrm{ml}^{-1}$ barley $\beta-1,3 ; 1,4$-glucan polymer dissolved in $100 \mathrm{mM}$ Sodium Phosphate buffer was hydrolysed with $0.05 \mathrm{U} \mathrm{ml}^{-1}$ lichenase (A and $B$ ) or $0.025 \mathrm{U} \mathrm{ml}^{-1}$ lichenase ( $\mathrm{C}$ and D) and the reaction was stopped upon $0,15,30,45,60,120$ or 240 min incubation. 14-day old in-vitro grown Arabidopsis Col-0 seedlings were treated for 30 min with $10 \mu \mathrm{g} \mathrm{mL}^{-1}$ chitin, $50 \mathrm{nM}$ flg22, $10 \mathrm{mM}$ Sodium phosphate buffer, $1 \mathrm{mg} \mathrm{ml}^{-1} \beta-1,3 ; 1,4-\mathrm{glucan}, 0.005 \mathrm{U} \mathrm{ml}^{-1}$ or $0.025 \mathrm{U} \mathrm{ml}^{-1}$ heat inactivated lichenase, $1 \mathrm{mg} \mathrm{ml}^{-1}$ $\beta$-1,3;1,4-glucan with $0.005 \mathrm{U} \mathrm{ml}^{-1}$ or $0.025 \mathrm{U} \mathrm{ml}^{-1}$ heat-treated lichenase or a 1:10 dilution of MLG oligosaccharides ( $\beta-1,3 ; 1,4$-glucan + active lichenase upon different incubation times). The expression of the defence genes WRKY33 (A and $\mathrm{C}$ ) and WRKY53 (B and D) was analysed via qRT PCR. UBIQUITIN5 served as reference gene. The bars represent means of two biological replicates with each three technical replicates. Error bars represent STDEV. Statistical significance is indicated with asterisks with not significant $(n s)=p>0.5$, ${ }^{*}=p \leq 0.5,{ }^{* *}=p \leq 0.001$ and ${ }^{* * *}=p \leq 0.001$. The unpaired student's t-teat was used to calculate $p$-values. 


\subsubsection{Hydrolysis products of the barley $\beta-1,3 ; 1,4-$ glucan polymer do not inhibit seedling growth}

Typically, seedlings show an inhibition of growth when they are exposed to high concentrations of elicitors e.g. flg22 or elf18 (Gómez-Gómez and Boller, 2000; Zipfel et al., 2006). The receptor for elf18 was identified in a reverse genetic screen in which several T-DNA insertion lines were tested for their sensitivity towards elf18 in seedling growth experiments (Zipfel et al., 2006). Similarly, if MLGs negatively influence seedling growth, receptors or co-receptors required for MLG perception could be identified with a forward genetic screen using the seedling growth assay.

To test whether MLG oligosaccharides can inhibit seedling growth, 5-day old in-vitro grown Col-0 seedlings were transferred to $1 / 2$ MS medium plus sucrose containing either no elicitor, Sodium phosphate buffer, flg22 or MLG oligosaccharides that were generated upon $\beta-1,3 ; 1,4-$ glucan hydrolysis (Figure S20). The seedlings were grown for eight further days and then, the size of the seedlings as well as the dry weight of the 13-day old seedlings was analysed. A dramatic reduction in growth was only directly observed for seedlings that were grown in the presence of the bacterial MAMP flg22 but not for seedlings grown in medium containing MLG oligosaccharides or Sodium phosphate buffer (Figure $22 \mathrm{~A}$ ). Furthermore, the dry weight of seedlings growing in medium containing MLG oligosaccharides was not significantly reduced in comparison to seedlings growing in $1 / 2$ MS plus sucrose medium without elicitor indicating that MLG oligosaccharides do not influence seedling growth (Figure $22 \mathrm{~B}$ ). Consequently, testing the sensitivity of several mutants or ecotypes to MLGs in a seedling growth assay cannot be used to identify molecular components required for MLG perception. 
A

$1 / 2 \mathrm{MS}+$ sucrose

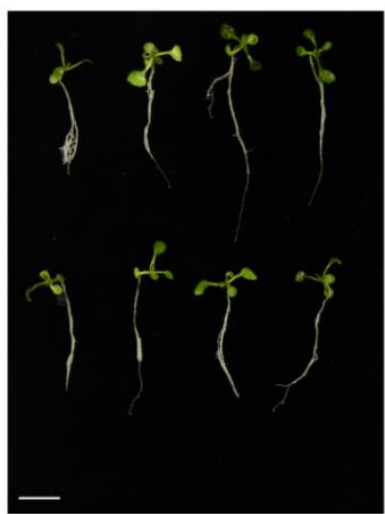

$1 / 2 \mathrm{MS}+$ sucrose $+$

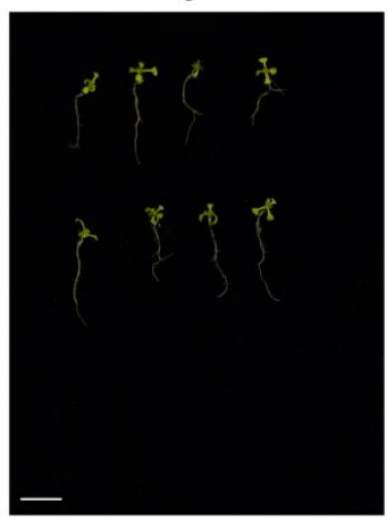

$1 / 2 \mathrm{MS}+$ sucrose

Sodium phosphate buffer

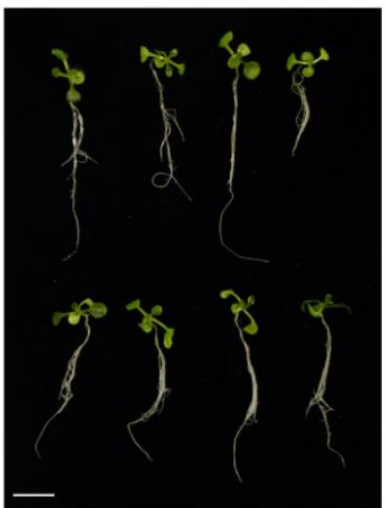

$1 / 2 \mathrm{MS}+$ sucrose

MLG oligosaccharides

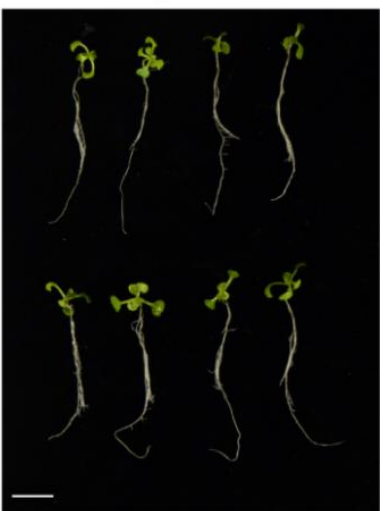

B

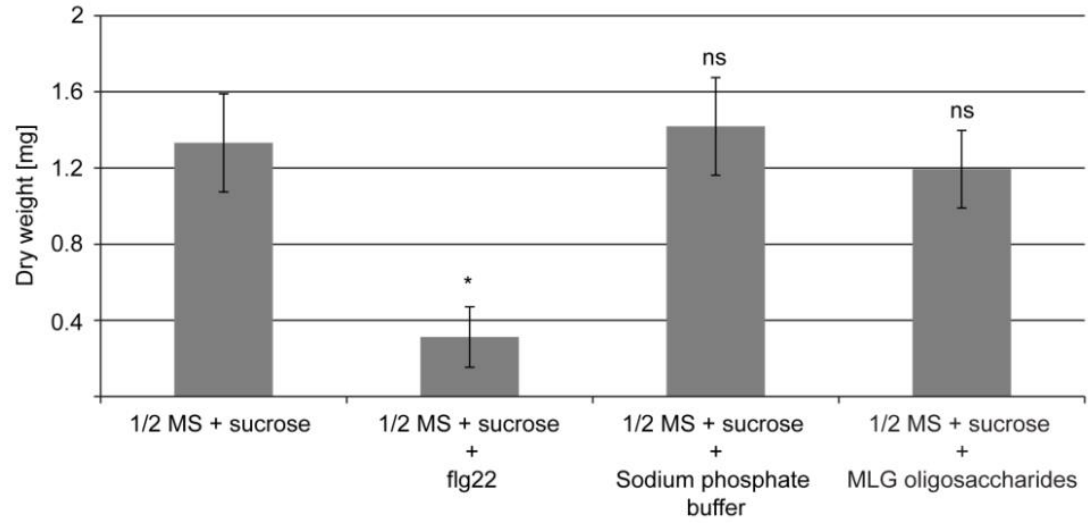

Figure 22. Effect of MLG oligosaccharides on seedling growth. To obtain MLG oligosaccharides of varying length, $10 \mathrm{mg} \mathrm{ml}^{-1}$ barley $\beta-1,3 ; 1,4$-polymer dissolved in $100 \mathrm{mM}$ Sodium phosphate buffer was hydrolysed with $1 \mathrm{U} \mathrm{ml}^{-1}$ lichenase and the reaction was stopped upon 1h. 5-day old in-vitro grown Arabidopsis Col-0 seedlings were transferred to liquid $1 / 2$ MS plus sucrose medium containing no elicitor, $10 \mathrm{mM}$ Sodium Phosphate buffer $(\mathrm{pH}=6.5), 1 \mu \mathrm{M}$ flg22 or a 1:10 dilution of MLG oligosaccharides and grown for 8 further days. (A) Pictures were taken of 13-day old seedlings. Scale bar represents $1 \mathrm{~cm}$. (B) 13-day old seedlings were dried and the weight of 7 to 8 seedlings per treatment was measured. The weight of one seedling was calculated by dividing the weight of all seedlings of one treatment by the total number of seedlings. Bars represent the average weight of one seedling of two biological replicated consisting of 7 to 8 seedlings each. Error bars represent STDEV. Statistical significance is indicated by asterisk with not significant (ns) $=p>0.5,{ }^{*}=p \leq 0.5,{ }^{* *}=p \leq 0.01$ and ${ }^{* * *}=p \leq 0.001$. To calculate $p$ values, the unpaired student's t-teat was used. 


\subsection{Molecular components involved in MLG perception could not be identified with reverse and forward genetic screens}

\subsubsection{MLG perception does not involve LysM domain containing RLK and RLPs}

So far, known PRRs are either RLKs or RLPs. RLKs that contain LysM domains were shown to be involved in the perception of oligosaccharide MAMPs, e.g. chitin and peptidoglycan (Macho and Zipfel, 2014). In Arabidopsis five genes encode for LysM domain containing RLKs (LYKs): LYK1/CERK1 and LYK2 to LYK5 (Zhang et al., 2007). The LysM RLKs CERK1, LYK4 and LYK5 are involved in perception of the fungal MAMP chitin whereas LYK2 does not seem to play a role in chitin perception (Miya et al., 2007; Petutschnig et al., 2010; Wan et al., 2012; Cao et al., 2014). Furthermore, CERK1 was shown to play a role in the perception of $\beta-1,3$-glucan oligomers as well as in bacterial peptidoglycan perception together with LYM1 and LYM3 (Willmann et al., 2011; Mélida et al., 2018).

To test the involvement of CERK1, LYK2, LYK4 and LYK5 in MLG perception, MAPK activation upon treatment with MLG oligosaccharides was monitored in the chitin receptor mutant cerk1-2 (GABI_096F09), the T-DNA insertion line lyk2-1 (SALK_152226) and the double mutant lyk5-2 lyk4-2 (SALK_131911C x GABI_897A10) via immunoblot analysis using the p44/42-antibody. MLG oligosaccharides were obtained from hydrolyzing barley $\beta-1,3 ; 1,4$-polymer with $1 \mathrm{U} \mathrm{ml}^{-1}$ lichenase. The hydrolysis was checked via TLC (Figure S20). Upon treatment with MLG oligosaccharides, MAPKs were phosphorylated in cerk1-2, lyk2-1 and the lyk5-2 lyk4-1 double mutant to the same level as in Col-0 suggesting that neither CERK1, LYK2, LYK4 nor LYK5 are involved in MAPK activation upon MLG perception (Figure $23 \mathrm{~A}$ and $\mathrm{B}$ ).

LYM2 mediates the decrease in molecular flux between cells through plasmodesmata in response to chitin (Faulkner et al., 2013). LYT1 is an extracellular LysM domain containing protein that was found to be co-regulated with CERK1 and might be involved in elicitor perception with CERK1 (Dr. Elena Petutschnig, personal communication). To address whether LYM2 or LYT1 are involved in the perception of MLGs, MAPK activation upon MLG oligosaccharide treatment in lym2-1 (SAIL_343_B03), lym2-4 (GABI-Kat 165 H02) and lyt1-1 (SALK_144729) was analysed with Western Blot using the p44/42-antibody. MAPK6 and MAPK3 phosphorylation was not compromised in lym2-1, lym2-4 or lyt1-1 indicating that LYM2 and LYT1 are not involved in MLG perception (Figure $23 \mathrm{~B}$ ). 
A

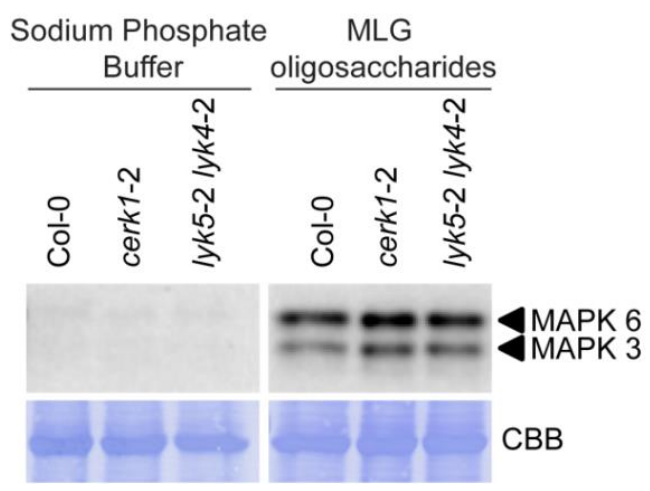

$\mathrm{B}$
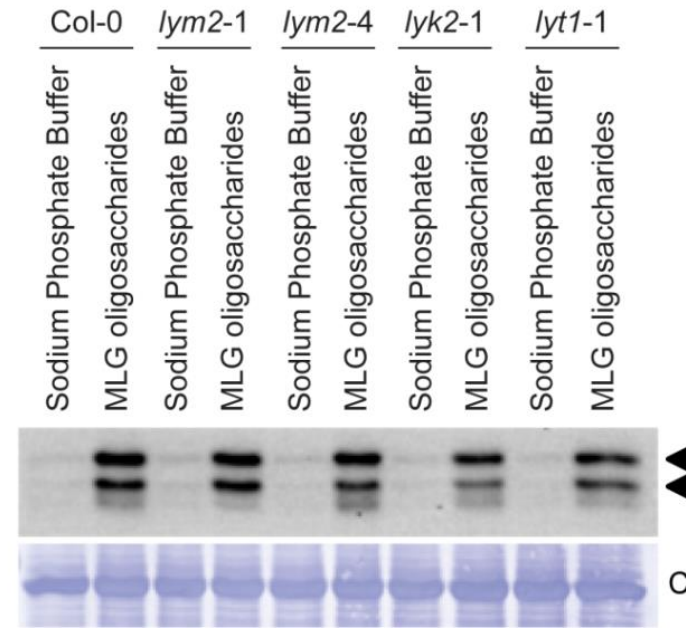

MAPK

MAPK 3

CBB

Figure 23. Activation of MAPK6 and MAPK3 in different LysM-RLKs and LysM-RLPs mutants upon MLG oligosaccharide treatment. To obtain MLG oligosaccharides of varying length, $10 \mathrm{mg} \mathrm{ml}^{-1}$ barley $\beta-1,3 ; 1,4-$ glucan polymer dissolved in $100 \mathrm{mM}$ Sodium phosphate buffer $(\mathrm{pH}=6.5)$ was hydrolysed with $1 \mathrm{U} \mathrm{ml}^{-1}$ lichenase and the reaction was stopped upon 1h. 14-day old in-vitro grown Arabidopsis seedlings of Col-0, cerk1-2 and lyk5-2 lyk4-1 (A) or Col-0, lym2-1, lym2-4, lyk2-1 or lyt1-1 (B) were treated for 12 min with $10 \mathrm{mM}$ Sodium phosphate buffer or a 1:10 dilution of MLG oligosaccharides. Activation of MAPK was analysed via Western Blot using p44/42 antibody. Lower panel shows Coomassie Brilliant Blue (CBB) staining as loading control. The experiment was performed once.

\subsubsection{LRR-RLKs are not involved in MLG perception}

The bacterial MAMPs flg and EF-TU induce a set of immune responses in $A$. thaliana and are perceived by the receptor kinases FLS2 and EFR, respectively (Chinchilla et al., 2006; Zipfel et al., 2006).

To test whether FLS2 and EFR are required in MLG perception, the activation of MAPK upon treatment with enzymatically generated MLG oligosaccharides (Figure S20) was tested in fls2c (SAIL_691_C4) and efr-1 (SALK_044334) via immunoblot analysis using the p44/42-antibody. Loss of FLS2 in the fIs2c mutant did not abolish MAPK activation upon MLG oligosaccharide treatment (Figure 24). Similarly, loss of EFR in the efr-1 mutant did not affect MAPK activation upon treatment with MLG oligosaccharides (Figure 24). These results indicate that neither FLS2 nor EFR are involved in MAPK activation upon MLG perception. 


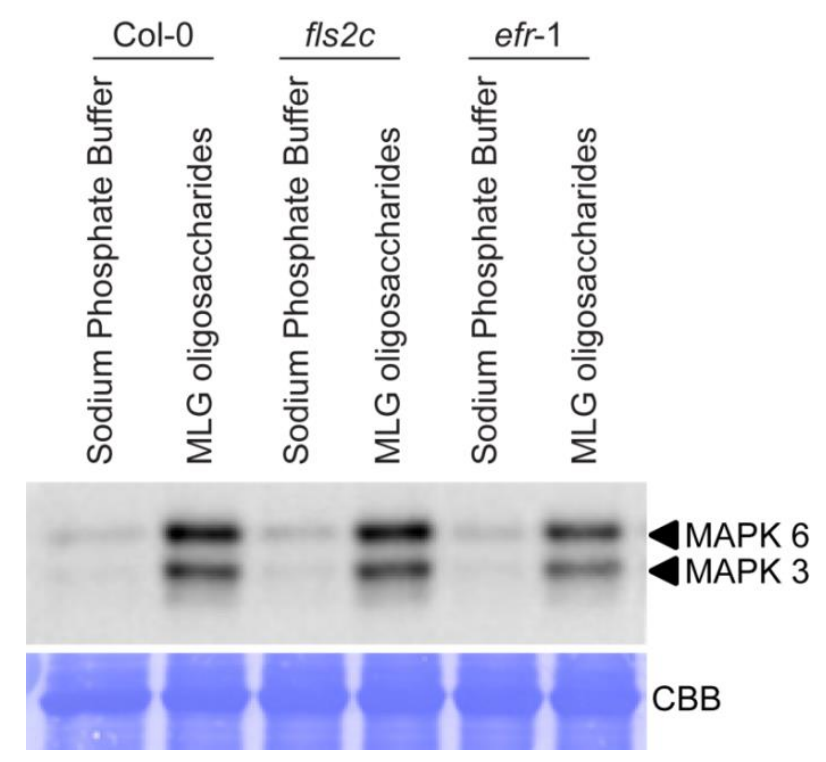

Figure 24. Activation of MAPK6 and MAPK3 in fIs2c and efr-1 upon MLG oligosaccharide treatment. To obtain MLG oligosaccharides of varying length, $10 \mathrm{mg} \mathrm{ml}^{-1}$ barley $\beta-1,3 ; 1,4$-polymer dissolved in $100 \mathrm{mM}$ Sodium phosphate buffer $(\mathrm{pH}=6.5)$ was hydrolysed with $1 \mathrm{U} \mathrm{ml}^{-1}$ lichenase and the reaction was stopped upon $1 \mathrm{~h}$. 14-day old in vitro grown Arabidopsis seedlings of Col-0, fls2c and efr-1 were treated for 12 min with $10 \mathrm{mM}$ Sodium phosphate buffer or a 1:10 dilution of MLG oligosaccharides. Activation of MAPK was analysed via Western Blot using p44/42-antibody. Lower panel shows Coomassie Brilliant Blue (CBB) staining as loading control. The experiment was performed once.

The LRR-RLK BAK1 is involved in brassinosteroid signaling and also plays a role as positive regulator in plant immunity (Li et al., 2002; Nam and Li, 2002; Heese et al., 2007; Chinchilla et al., 2007). BAK1 is required for early immune responses induced by flg22 and elf18 and was shown to form a complex with the respective receptors FLS2 and EFR (Heese et al., 2007; Chinchilla et al., 2007; Roux et al., 2011). Furthermore, BAK1 is involved in PEPR1/2-dependent responses that are the corresponding receptors for the Pep DAMP molecules. These results demonstrate that BAK1 is involved in multiple PRR-signalling pathways (Roux et al., 2011).

To test a potential involvement of BAK1 as co-receptor in the perception of MLGs, the activation of MAPK was monitored over time in bak1-4 and bak1-5 upon MLG oligosaccharide treatment. Bak1-4 (SALK_116202) is a knock-out mutant, while bak1-5 harbours a single amino acid exchange in the $10^{\text {th }}$ exon and is specifically impaired in PTI responses (Schwessinger et al., 2011). Activation of MAPKs was neither reduced nor delayed in bak1-4 and bak1-5 in comparison to Col-0 upon treatment with MLG oligosaccharides of varying length (Figure $25 \mathrm{~A}$ ) that were enzymatically generated using the commercially available B. subtilis lichenase (Figure S20). This suggests that the co-receptor BAK1 is not involved in MLG perception.

The LRR-RLK SOBIR1 plays a role in activating defence responses by certain immune receptors. The RLP RLP30 is involved in perception of the peptide effector SCFE1 and requires SOBIR1 (Zhang et al., 2013). Furthermore, SOBIR1 is indispensable for RBPG1-mediated responses towards fungal polygalacturonases (Zhang et al., 2014). 
To test the involvement of SOBIR1 in MLG perception, MAPK activation upon MLG oligosaccharide treatment was analysed in the two knock-out mutants sobir1-12 (SALK_050715) and sobir1-14 (GABI-Kat_643F07). The activation of MAPK in the two different mutants sobir1-12 and sobir1-14 was not impaired after MLG elicitation indicating that SOBIR1 is not involved in perception of MLGs (Figure 25 B).

A
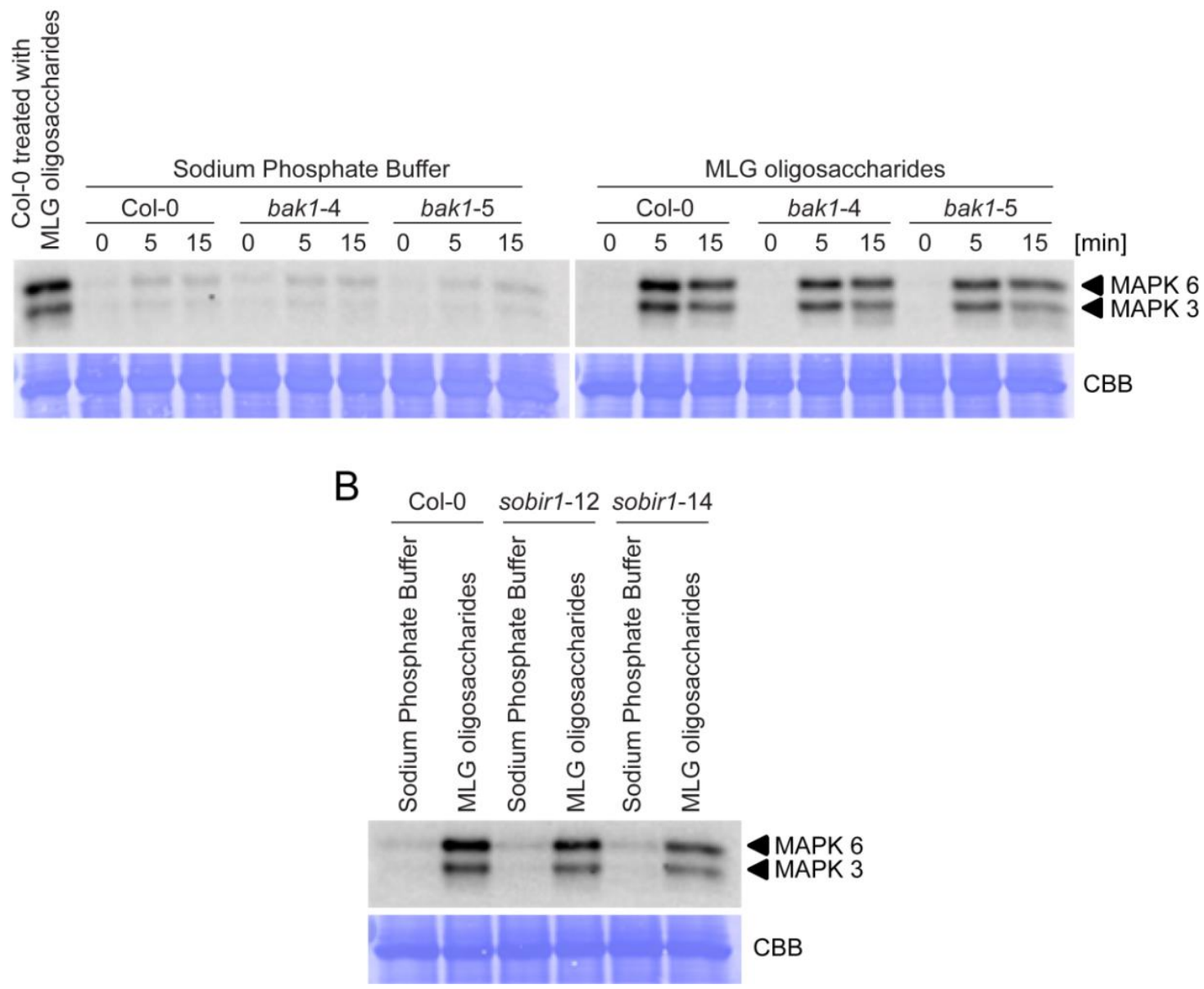

Figure 25. Activation of MAPK6 and MAPK3 in different co-receptor mutants upon MLG oligosaccharide treatment. To obtain $\beta$-glucan oligosaccharides of varying length, $10 \mathrm{mg} \mathrm{ml}^{-1}$ barley $\beta-1,3 ; 1,4$-polymer dissolved in $100 \mathrm{mM}$ Sodium phosphate buffer was hydrolysed with $1 \mathrm{U} \mathrm{ml}^{-1}$ lichenase and the reaction was stopped upon 1h. (A) MAPK activation in bak1-4 and bak1-5. 14-day old in-vitro grown seedlings of Col-0, bak1-4 and bak1-5 were treated with $10 \mathrm{mM}$ Sodium Phosphate buffer or a 1:10 dilution of MLG oligosaccharides for 0,5 or $15 \mathrm{~min}$. MAPK activation was analysed via Western Blot using p44/42 antibody. Lower panels show Coomassie Brilliant Blue (CBB) staining as loading control. The experiment was performed once. (B) MAPK activation in sobir1-12 and sobir1-14. 14-day old in-vitro grown seedlings of Col-0, sobir1-12 and sobir1-14 were treated with $10 \mathrm{mM}$ Sodium phosphate buffer or a 1:10 dilution of MLG oligosaccharide for $12 \mathrm{~min}$. MAPK activation was analysed via Western Blot using p44/42 antibody. Lower panels show Coomassie Brilliant Blue (CBB) staining as loading control. The experiment was performed once. 


\subsubsection{7 tested Arabidopsis ecotypes are MLG-sensitive}

The plant species $A$. thaliana exhibits a high variability regarding their sensitivity towards different MAMPs due to distinct ecotype-specific receptor repertoires (Gómez-Gómez and Boller, 2000; Zhang et al., 2013; Albert et al., 2015). This has been exploited in the past to identify MAMP-receptors e.g. the SCFE1 receptor RLP30 was identified with the help of the insensitive accessions Lov-1, Mt-0 and Sq-1 (Zhang et al., 2013).

In order to identify molecular components required for MLG perception, MLG-insensitive and MLG-sensitive ecotypes should be identified and subsequently, be used to identify the corresponding receptor using map-based cloning strategies. A set of 527 Multiparent Advanced Generation Inter-Cross (MAGIC) lines, derived from crossing 19 parental ecotypes, is available that can be used to identify e.g. novel PRRs via map-based cloning. If MAMP-sensitive and MAMP-insensitive parental lines can be identified, the respective MAGIC lines can be used to identify the corresponding receptor (Kover et al., 2009). To identify MLG-sensitive and MLG-insensitive Arabidopsis accessions and potentially the MLG receptor or co-receptors, all parental ecotypes of the MAGIC lines, except for Ler- 0 , were analysed regarding the phosphorylation of MAPK upon MLG elicitation. The B. subtilis lichenase was used to enzymatically produce oligomeric MLGs (Figure S20). The immunoblot analysis revealed that all tested parental lines showed MAPK activation upon MLG oligosaccharide treatment (Figure 26). Although the MAPK activation for the ecotype Zu-0 was less pronounced in comparison to the other ecotypes, this result indicates that all of these ecotypes harbour the molecular components involved in MLG perception (Figure 26).

Since the parental lines of the MAGIC lines did not reveal an ecotype that could not respond to MLGs, 110 further ecotypes were tested for MAPK activation upon MLG oligosaccharide elicitation. However, MAPK activation was not impaired in one of the 110 tested ecotypes in response to MLG oligosaccharides indicating that the tested ecotypes are sensitive towards MLGs and contain the molecular components required for MLG perception (Figure S21, Figure S22, Figure S23). In conclusion, none of the 127 tested ecotypes can be used to identify components of the MLG perception system. 

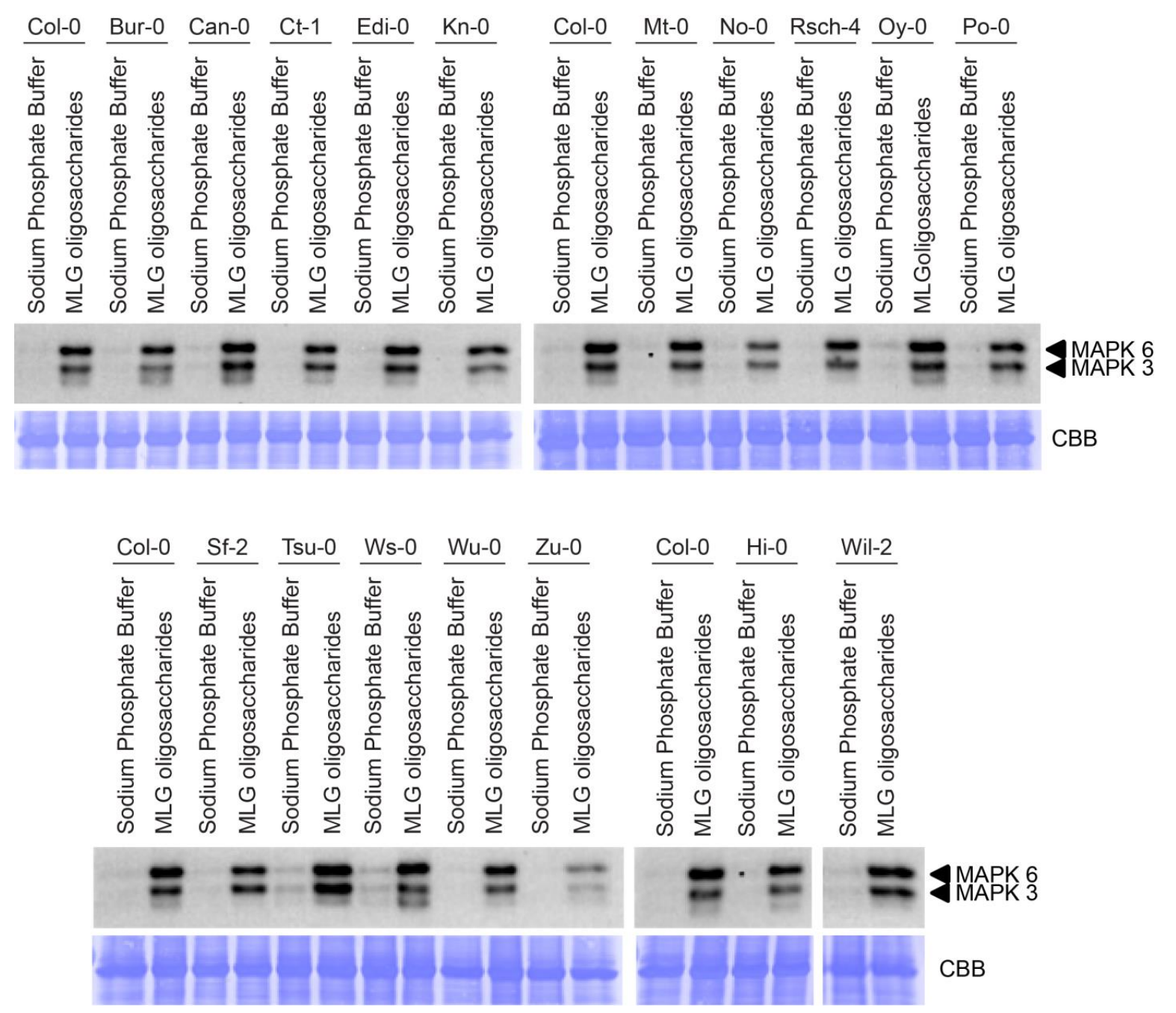

Figure 26. MAPK activation in the parental ecotypes of the MAGIC lines. To obtain MLG oligosaccharides of varying length, $10 \mathrm{mg} \mathrm{ml}^{-1}$ barley $\beta-1,3 ; 1,4-$ polymer dissolved in $100 \mathrm{mM}$ Sodium Phosphate buffer was hydrolysed with $1 \mathrm{U} \mathrm{ml}^{-1}$ lichenase and the reaction was stopped upon 1h. 14-day old in-vitro grown seedlings of the respective ecotypes were treated with either $1 \mathrm{mM}$ Sodium Phosphate buffer or a 1:10 dilution of MLG oligosaccharides for $12 \mathrm{~min}$. Activation of MAPK was analysed via Western Blot using the p44/42-antibody. Lower panel shows Coomassie Brilliant Blue (CBB) staining as loading control. The experiment was performed once. 


\section{Discussion}

The first physical barrier that pathogens encounter is the plant cell wall. In order to successfully infect a plant, pathogens need to overcome this physical barrier and evolved several mechanisms to do so. Fungal penetration structures called appressoria are used to penetrate the cell wall with pressure (Chisholm et al., 2006). Furthermore, secreted CWDEs have been shown to be involved in cell wall degradation and pathogenicity of necrotrophic and hemibiotrophic pathogens. For example, simultaneous knockdown of ten genes encoding for xylanases of the hemibiotrophic fungus Magnaporthe oryzae resulted in a reduced penetration rate and a reduction in lesion size on barley and wheat (Nguyen et al., 2011). So far, however, the involvement of CWDEs in the pathogenicity of biotrophic pathogens has not been elucidated. The first part of this study focuses on the functional characterization of $B g h$ enzymes that might be involved in pathogenicity.

\subsection{Identification and functional characterization of fungal CWDEs}

\subsubsection{Genomic and transcriptomic data reveal GH family 17 as potentially involved in pathogenicity}

A genome analysis conducted by our collaborators at the IPK Gatersleben (research group of the late Dr. Patrick Schweizer) identified a total of 75 CWDEs in the genome of Bgh. The majority of these proteins (62) were designated to 18 different GH families and one Auxiliary Activity family that was formerly described as GH family 61 (unpublished data, Dr. Patrick Schweizer, personal communication). The result of this analysis is contradictory to a genome analysis conducted in 2010 in which only seven CWDEs were identified and designated to two different GH families and one Auxiliary Activity family (Spanu et al., 2010). However, analysis of the protein sequences of the 62 GHs for carbohydrate active enzyme domain signatures verified 60 of the proteins indicating that the genome of Bgh contains more CWDEs as previously thought (Table S1). The presence of $60 \mathrm{GHs}$ and in total 72 CWDEs in Bgh nevertheless supports the finding of a study observing a reduced number of CWDEs in biotrophic pathogens compared to necrotrophic or hemibiotrophic pathogens (Zhao et al., 2014). For example, the biotrophic plant pathogen Ustilago maydis contains 238 CWDE-encoding genes in its genome, while the hemibiotrophic rice blast fungus $M$. oryzae contains 522 genes encoding for CWDEs and the necrotrophic pathogen Verticillium dahliae genome harbours 545 genes for CWDEs (Zhao et al., 2014).

In order to identify $\mathrm{GH}$ families that are involved in pathogenicity, the expression of the 60 identified GHs were analysed using publicly available transcriptomic data (Hacquard et al., 2013). This analysis revealed that members of five different $\mathrm{GH}$ families including $\mathrm{GH} 5, \mathrm{GH} 16, \mathrm{GH} 17, \mathrm{GH} 47$ and GH76 were highly up-regulated during the first $24 \mathrm{~h}$ of the infection indicating their potential importance in the infection process (Table S2). Upon fungal attack, the plant cell wall is reinforced. For example, papillae are formed in immediate proximity to fungal penetration sites as observed in barley upon $\mathrm{Bgh}$ attack (Underwood, 2012). Papillae are mainly composed of callose, a polymer of $\beta-1,3$-linked 
glucose monomers. As Bgh has to overcome the plant cell wall and defence-associated papillae for a successful infection, $\beta$-1,3-glucan degrading enzymes might be involved in pathogenicity. Of the five different $\mathrm{GH}$ families, the family $\mathrm{GH} 17$ was chosen for further functional characterization due to its designated substrate specificities towards $\beta$-1,3-glucans (Hrmova and Fincher, 1993; Carbohydrate Active Enzymes database, http://www.cazy.org/, Lombard et al., 2014). Additionally, analysis of the protein sequences of all seven members of the GH family 17 revealed that all proteins contain either a canonical $\mathrm{N}$-terminal signal peptide or an unconventional secretion signal indicating that all proteins are secreted into the plant apoplast and potentially act on the plant cell wall. As two of the seven GH17 members showed an enhanced penetration rate in HIGS analysis indicating that they negatively affect pathogenicity, these two members were excluded from further analysis and only five Bgh GH17 members will be functionally analysed.

\subsubsection{Only BGH06777 could be expressed and purified using the $\boldsymbol{P}$. pastoris expression system}

For functional characterization of the five selected $\mathrm{Bgh}$ GH17 members, all five genes should be expressed and consequently, purified using the $P$. pastoris expression system. No distinct signals at the expected sizes could be detected for BGH00219, BGH00220, BGH00734 or BGH00736 in the supernatant of the cultures of different clones grown under four different conditions (Figure S1 A, B, $C, D)$. There are several reasons why these proteins might not be expressed. One explanation could be that the GH17 proteins are susceptible to extracellular neutral pH proteases and are proteolytically degraded. This was also observed for gelatins produced in P. pastoris. The degradation of gelatins could be minimized by performing the expression at $\mathrm{pH} 3$ (Werten et al., 1999). To test whether BGH00219, BGH00220, BGH00734 or BGH00736 are degraded by extracellular proteases, the expression of the four $\mathrm{GH} 17$ members could also be performed at $\mathrm{pH} 3$. To induce the expression of genes in P. pastoris, methanol is added to the culture. However, methanol does not only induce expression of the desired gene but was also shown to activate the generation of proteases in $P$. pastoris and cell lysis in high density cultures. Consequently, proteases that are found intracellular are present in the medium upon cell lysis and can degrade the secreted protein (Sinha et al., 2005). It might be possible that during the expression of Bgh00219, Bgh00220, Bgh00734 and Bgh00736, several proteases were generated and released into the medium due to cell lysis in a high density culture. To prevent proteolytic degradation, the optimal induction times as well as methanol feeding strategies have to be determined (Sinha et al., 2005). Alternatively, protease inhibitors could be added to the medium. Another explanation for the absence of the four proteins in the supernatant could be that the genes of interest might not be transcribed due to the presence of multiple AT rich regions (Gurkan and Ellar, 2003). To test whether the respective genes are expressed in P. pastoris, mRNA could be extracted and semi-quantitative PCR or qRT-PCR could be performed.

A distinct signal, however, could be obtained for BGH06777 at the expected size in all tested clones (Figure S1 E). The clone that showed the strongest signal for BGH06777 was chosen to express and 
purify the protein in high amounts. The recombinant purified protein was then subject for further biochemical characterization.

\subsubsection{BGH06777 was glycosylated by $P$. pastoris}

Of the five selected GH17 proteins, only BGH06777 could be expressed and purified in high amounts from $P$. pastoris. In SDS gel electrophoresis experiments the recombinant protein migrated at a size of approximately $35 \mathrm{kDa}$ (Figure S2), which fits well to the predicted molecular weight of $32.9 \mathrm{kDa}$. Additional mass spectrometry analysis detected masses of the purified protein in the range between $35 \mathrm{kDa}$ and $36.9 \mathrm{kDa}$ (Figure $4 \mathrm{~A}$ ). P. pastoris is known to $\mathrm{N}$-glycosylate proteins at an asparagine residue in the sequence asparagine-x-threonine/serine where $x$ can be any amino acid (Bretthauer and Castellino, 1999). An online tool for the prediction of N-glycosylation sites (http://www.cbs.dtu.dk/services/NetNGlyc/) revealed one glycosylation site for BGH06777 at the asparagine at position 191 (Table 18) suggesting that the difference in size as well as the occurrence of different masses may be due to glycosylation. P. pastoris was shown to have $\mathrm{N}$-glycosylation of a high-mannose type with mainly 8-14 mannose residues per chain but also longer chains of up to 30 mannose residues have been found. Furthermore, the $\mathrm{N}$-linked oligosaccharides can also be phosphorylated (Bretthauer and Castellino, 1999). Thus, the masses identified in mass spectrometry likely correspond to BGH06777 with either N-linked oligosaccharides with 11-20 mannose residues or $\mathrm{N}$-linked phosphorylated oligosaccharides containing 13-18 mannose residues. PNGase $\mathrm{F}$ is an amidase that cleaves $\mathrm{N}$-linked oligosaccharides from glycoproteins (Plummer et al., 1984). Treatment of BGH06777 with PNGase F resulted in the migration of BGH06777 at approximately $33 \mathrm{kDa}$ (Figure S2) and the identification of a mass at $32.97 \mathrm{kDa}$ in mass spectrometry (Figure $4 \mathrm{~B}$ ) which supports the hypothesis that BGH06777 was glycosylated. N-glycosylation is a co- and post-translational modification that occurs in all eukaryotes (Strasser, 2016) and thus, it is likely that BGH06777 is also glycosylated in Bgh. $\mathrm{N}$-glycosylation fulfills a variety of different functions including induction of proper folding and biological activity as well as preventing proteolytic degradation of the respective protein (Rayon et al., 1998). Recently, the role of N-linked glycans on the enzymatic activity of a xylanase designated as GH10 from A. fumigatus has been studied (Chang et al., 2017). The deglycosylated protein exhibited the same $\mathrm{pH}$ optimum as the glycosylated protein, however, the $\mathrm{pH}$ range in which it showed activity was narrower. Furthermore, the thermal stability as well as the biological activity was lower for the deglycosylated protein in comparison to the glycosylated protein. These results further supported the positive effect of $\mathrm{N}$-glycosylation on enzyme stability (Chang et al., 2017). To study the effect of glycosylation on BGH06777, the deglycosylated protein could also be functionally characterized in future experiments. The glycosylated form of BGH06777 was used in the following detailed biochemical characterization. To our knowledge, this is the first GH17 protein from the powdery mildew Bgh studied so far. 


\subsubsection{BGH06777 is a typical GH17 $\beta-1,3-g l u c a n a s e$}

Substrate analysis revealed that BGH06777 hydrolysed $\beta$-1,3-glucan oligosaccharides with a minimum length of four glucose monomers (Figure 5) demonstrating that BGH06777 is an active $\beta-1,3$-glucanase and that binding of at least four monomers is required for efficient hydrolysis. The hydrolysis products generated by BGH06777 were oligosaccharides with a length of two to four glucose monomers. This finding is in agreement with previous studies about substrate specificities of other enzymes designated as GH17. Three $\beta-1,3$-glucan endohydrolases from barley were shown to hydrolyse the $\beta$-1,3-glucan polymer laminarin and the main hydrolysis products for two of the isoenzymes were laminaribiose and laminaritriose (Hrmova and Fincher, 1993). A GH17 enzyme from banana fruit was also shown to be active on laminarin (Peumans et al., 2000). Additionally, the GH17 $\beta-1,3$-glucanosyltransferase Bgt2p of $A$. fumigatus was shown to cleave laminaribiose from a $\beta-1,3$-glucan chain (Gastebois et al., 2010).

The hydrolytic activity of BGH06777 on $\beta$-1,3-glucan oligosaccharides can further be described kinetically. The substrate concentration at which the half-maximal reaction rate is achieved is described by the Michaelis constant $\mathrm{K}_{\mathrm{M}}$ (Michaelis and Menten, 1913). $\mathrm{K}_{\mathrm{M}}$ is influenced by the binding affinity of a substrate to the enzyme as well as the rate of product formation. Thus, $\mathrm{K}_{M}$ is only an approximate measure of binding affinity and can only be used as measure for binding affinity if binding of the substrate to the enzyme is slower than formation of the enzyme-substrate complex and the product (Kessel and Ben-Tal, 2018). The $\mathrm{K}_{\mathrm{M}}$ value of BGH06777 for laminarihexaose was slightly higher than the $K_{M}$ value for laminaripentaose (Table 19). Under the assumption that product formation occurs faster than binding of the substrate to the enzyme, the obtained $K_{M}$ values indicates that BGH06777 has a lower affinity for laminarihexaose than for laminaripentaose. However, during hydrolysis of the $\beta-1,3$-glucan hexamer laminaribiose and laminaritetraose are generated. The generated product laminaritetraose might act as additional substrate during $\beta$-1,3-glucan hexamer degradation and might therefore influence the $\mathrm{K}_{\mathrm{M}}$ value obtained for laminarihexaose. On the contrary, products generated during laminaripentaose hydrolysis do not represent potential substrates for BGH06777 and thus, might not influence the $K_{M}$ value. Nevertheless, the obtained $K_{M}$ values are similar to reported $K_{M}$ values for the three barley $\beta$-1,3-glucan endohydrolases (Hrmova and Fincher, 1993). The turnover number $k_{\text {cat }}$ describes the number of substrate molecules that are converted into products per active site per time unit (Michaelis and Menten, 1913). $\mathrm{K}_{\text {cat }}$ obtained for laminarihexaose degradation was about 5 -fold higher compared to laminaripentaose degradation suggesting that BGH06777 favors longer oligosaccharide chains (Table 19). This is also supported by the observation that the catalytic efficiency was higher for laminarihexaose degradation than for laminaripentaose degradation (Table 19). This further suggests that the active site of the enzyme contains a minimum of six subsites. The active site of a GH can be labelled with negative subsites (-n) away from the cleavage site to the non-reducing end and with positive subsites $(+n)$ away from the cleavage site towards the reducing end of a sugar. The glycosidic linkage of the oligosaccharide bound to the enzyme is hydrolysed between the subsites -1 and +1 (Davies et al., 1997). ${ }^{18} \mathrm{O}$ labelling of the product and subsequent mass spectrometry revealed that BGH06777 contains in total six subsites 
with four negative and two positive subsites (Figure 9, Table 20). The length of the active site of BGH06777 is comparable to active sites of other GH17 enzymes. The three barley $\beta$-1,3-glucan endohydrolases were shown to contain eight subsites ranging from -3 to +5 (Hrmova et al., 1995) while the crystal structure of a tomato $\beta$-1,3-glucan endohydrolases revealed a total of 6 subsites with two negative and four positive subsites (Wojtkowiak et al., 2013). The crystal structure of a $\beta-1,3$-glucanosyltransferase from the fungus Rhizomucor miehei designated as a GH17 revealed a total of 5 subsites ranging from -3 to +2 (Qin et al., 2015). The number of negative and positive subsites determines the catalytic mode of an enzyme, thus, BGH06777 has a $-4 /+2$ catalytic mode. This catalytic activity indicates that the product laminaribiose is released from the reducing end of the respective substrate.

The catalytic activity of BGH06777 was shown to be optimal at a pH of 5.5 and in a temperature range from $25^{\circ} \mathrm{C}$ to $51^{\circ} \mathrm{C}$ (Figure 6). The obtained $\mathrm{pH}$ optimum is similar to reported $\mathrm{pH}$ optima of other $\mathrm{GH} 17$ enzymes. While the three barley $\beta$-1,3-glucan endohydrolases have an optimal $\mathrm{pH}$ at 4.8 (Hrmova and Fincher, 1993), a $\beta$-1,3-glucanase from olive exhibits its highest activity in a pH range from 4.5 to 6 (Huecas et al., 2001). Taken together, these data suggest that BGH06777 is a typical $\beta$-1,3-glucanase.

\subsubsection{BGH06777 might be involved in papillae degradation}

In this study, the biological function of BGH06777 could not be elucidated. However, the pH optimum of BGH06777 in addition to the presence of the signal peptide supports the idea that BGH06777 is active in the apoplastic space. The $\mathrm{pH}$ of the plant apoplast was shown to be between 5 and 6.5 (Grignon and Sentenac, 1991; Felle, 2006) and therefore corresponds to the $\mathrm{pH}$ optimum of BGH06777. Furthermore, plants generate callose-rich papillae upon fungal penetration that are thought to hinder further penetration (Underwood, 2012). Callose is a $\beta-1,3$-linked homopolymer of glucose monomers and thus represents a potential substrate for BGH06777. It might be possible that BGH06777 is involved in degrading papillae. A comparison of papillae that were either effective or ineffective in preventing $\mathrm{Bgh}$ penetration showed that ineffective papillae contain less callose, cellulose and arabinoxylan and lack an outer layer consisting of cellulose and arabinoxylan (Chowdhury et al., 2014). Consequently, it is conceivable to postulate that ineffective papillae might be overcome by Bgh employing a combination of pressure and enzymatic degradation of callose by BGH06777 and other GH17 enzymes. In contrast, the outer layer of cellulose and arabinoxylan present in effective papillae might provide the structural strength to prevent Bgh penetration, and may be due to lack of CWDEs with efficient cellulose and arabinoxylan hydrolytic activity. 


\subsubsection{Conclusion}

In the first part of this study, CWDEs involved in pathogenicity of $\mathrm{Bgh}$ were identified and functionally characterized. Analysis of genomic and transcriptomic data indicated that the family $\mathrm{GH} 17$ of $\mathrm{Bgh}$ might be involved in pathogenicity. The $\mathrm{Bgh}$ GH17 member BGH06777 was expressed and purified from $P$. pastoris and functionally characterized. To our knowledge, this is the first CWDE of $B g h$ that was characterized. BGH06777 hydrolyses $\beta$-1,3-glucans with a minimum length of four glucose monomers and the respective products are released from the reducing end of the sugar. The enzyme is most active in a temperature range from $25^{\circ} \mathrm{C}$ to $51^{\circ} \mathrm{C}$ and at a pH of 5.5 which corresponds to the $\mathrm{pH}$ in the plant apoplast.

\subsubsection{Outlook}

The glycosylated protein BGH06777 was heterologously expressed in P. pastoris and functionally characterized. To test the effect of glycosylation on the biological activity of BGH06777, the recombinant protein could be deglycosylated enzymatically and functionally characterized regarding $\mathrm{pH}$ and temperature optimum. Furthermore, the kinetic parameters $\mathrm{K}_{\mathrm{M}}$ and $\mathrm{k}_{\mathrm{cat}}$ as well as the catalytic efficiency could be determined. A narrower $\mathrm{pH}$ and temperature range as well as a lower catalytic efficiency would indicate a positive impact of $\mathrm{N}$-glycosylation on the biological activity of BGH06777. Furthermore, it would suggest that BGH06777 is present in the glycosylated form in Bgh.

The biological role of BGH06777 could not be identified during the course of this project. To substantiate the preliminary HIGS experiments conducted in the lab of our collaboration partner, the late Dr. Schweizer (IPK Gatersleben), which had suggested a potential involvement in pathogenicity, these should be repeated with a significant number of replicates. A reduced penetration rate compared to the wild-type empty HIGS vector control experiments would suggest an involvement of BGH06777 on pathogenicity while no change in the penetration rate would indicate that BGH06777 does not play a role. Previously it was shown that silencing of a single CWDE has no effect on the severity of the infection while simultaneous HIGS of several genes of one CWDE family resulted in reduced pathogenicity (Gómez-Gómez et al., 2002; Wu et al., 2006; Nguyen et al., 2011). This effect might be explained with a redundant function of CWDEs of the same family (Nguyen et al., 2011). Analogous experiments could be used in the future to address the question whether or not GH17 members of $B g h$ act redundantly and thus jointly contribute to pathogenicity.

Of course, it would also be interesting to functionally characterize the other members of the GH17 family, namely BGH00219, BGH00220, BGH00734 and BGH00736. Therefore, further P. pastoris clones should be generated and tested for the integration of the respective gene in the genome. Next, the expression of the respective gene should be tested. To circumvent possible proteolytic degradation, the expression could be performed at a $\mathrm{pH}$ of 3 and/or the medium could further be supplemented with a protease inhibitor mix. If a protein can be successfully purified, it can be 
functionally characterized concerning substrate specificity, $\mathrm{pH}$ optimum, temperature optimum and Michaelis-Menten kinetics.

Overall, the proposed experiments would shed light into the substrate specificities of GH17 enzymes of $B g h$ and their potential biological role.

\subsection{Identification of new cell-wall derived DAMP molecules}

Plants evolved several mechanisms to induce immune signaling upon pathogen attack. The perception of non-self MAMP molecules by PRRs leads to the activation of defence responses. Similarly, perception of DAMPs, self-molecules that are only abundant upon e.g. wounding or cell damage, triggers the induction of immune responses (Jones and Dangl, 2006; Boller and Felix, 2009). The action of CWDEs on the plant cell wall might release cell-wall oligosaccharides (Bacete et al., 2018). It is likely that plants are able to perceive the released cell wall oligosaccharide as DAMPs which leads to the initiation of defence responses. The second part of this study focuses on the identification of new cell-wall derived DAMP molecules in Arabidopsis and barley and the corresponding signaling components.

\subsubsection{Cellohexaose, xylohexaose and linear $\beta-1,3-$ glucan oligosaccharides could slightly induce immune responses in Arabidopsis}

In this study, a screen to identify new cell-wall derived DAMP molecules was conducted with a collection of 41 poly- and oligosaccharides. The majority of poly- and oligosaccharides did not activate immune responses (Figure S3, Figure S4, Figure S5, Figure S6, Figure S7, Figure S8, Figure S9), whereas eight of the ordered substances did trigger the influx of $\mathrm{Ca}^{2+}$ and/or activation of MAPK in Arabidopsis (Figure 10, Figure 11, Figure 12, Figure S10, Figure S11, Figure S12, Figure S13, Figure S14, Figure S15). These data suggested that plants evolved the ability to perceive some, but not all cell wall derived oligosaccharides that might be generated during pathogen attack.

\subsubsection{Arabidopsis can perceive cellulose-derived oligomers}

The cellulose derivative cellohexaose did not induce the generation of ROS but induced a slight influx of $\mathrm{Ca}^{2+}$ as well as the activation of MAPK in Arabidopsis (Figure S10, Figure S11) indicating that cellohexaose can be perceived by Arabidopsis which subsequently leads to the activation of immune responses. The observation that cellohexaose did not trigger the generation of ROS is in agreement with a previous study revealing that cellohexaose treatment did also not result in the generation of ROS in V. vinifera (Aziz et al., 2003). Cellulose is a major component of the cell wall of dicot plants and cellohexaose might be generated upon degradation of cellulose by microbial cellulose degrading 
enzymes. The degradation of cellulose by ascomycete and basidiomycete fungi involves at least three

different enzyme classes, namely endo- $\beta$-1,4-glucanases, exo- $\beta-1,4-$ glucanases or cellobiohydrolases and $\beta$-glucosidases (Baldrian and Valášková, 2008; Van Den Brink and De Vries, 2011; Glass et al., 2013). These three enzyme classes can be categorized in a minimum of 12 different $\mathrm{GH}$ families including $\mathrm{GH} 3, \mathrm{GH} 5, \mathrm{GH} 6$ as well as $\mathrm{GH} 7$ and are commonly found in hemibiotrophic and necrotrophic pathogens (King et al., 2011; Kubicek et al., 2014; Carbohydrate Active Enzymes database, http://www.cazy.org/, Lombard et al., 2014). Endo- $\beta$-1,4-glucanases cleave internal $\beta$-1,4-linkages within the cellulose chain leading to the release of $\beta$-1,4-glucan oligosaccharides of variable length, whereas exo- $\beta-1,4$-glucanases cleave the cellulose chain at the end of the chain thereby releasing cellobiose (Baldrian and Valášková, 2008; Van Den Brink and De Vries, 2011; Glass et al., 2013). The oligosaccharides as well as cellobiose are degraded into glucose monomers through the action of $\beta$-glucosidases (Baldrian and Valášková, 2008; Van Den Brink and De Vries, 2011). The products generated by endo-1,4- $\beta$-glucanases might include oligosaccharides with six glucose monomers further suggesting that cellohexaose acts as a DAMP in Arabidopsis. The finding that cellohexaose activates plant immune responses confirms observations from a study that was published during the course of this project. This study showed that cellobiose acts as an elicitor of defence responses and that cellotriose as well as cellotetraose induce the expression of the defence gene WRKY30 (de Azevedo Souza et al., 2017) but exclude further analysis of molecular components involved in cellulose-derived oligomer perception. The data generated in this study and from the publication by Shauna Somerville's research group suggest that cellulose degradation products can be perceived by plants and induce the activation of immune responses.

\subsubsection{Xylohexaose elicitation induces the activation of MAPK}

Treatment of Arabidopsis with the hemicellulose derivative xylohexaose resulted in the activation of MAPKs (Figure S13 B). As the induction of MAPK was only slight and treatment with xylohexaose did not result in a calcium response and a detectable generation of ROS (Figure S12, Figure S13), this carbohydrate was excluded from further analysis. A reason for the non-conclusive results could be the used carbohydrate concentration. To test the elicitor activity of xylohexaose, 10 or $100 \mu \mathrm{g} \mathrm{ml}^{-1}$ were used in the conducted assays. However, to detect the induction of defence responses upon ß-1,3-glucan oligosaccharide treatment, $250 \mathrm{mg} \mathrm{ml}^{-1}$ were used (Mélida et al., 2018). Furthermore, a strong induction of MAPK activation in $V$. vinifera and Arabidopsis is observed upon treatment with 0.5 and $1 \mathrm{mg} \mathrm{ml}^{-1}$ xyloglucan oligosaccharides (Claverie et al., 2018). This indicates that a high DAMP concentration is needed to obtain detectable readouts suggesting that the respective receptors have a low affinity towards the respective ligands (de Azevedo Souza et al., 2017). However, it is not known whether these high concentrations are of physiological relevance and can be achieved during pathogen invasion. It might also be possible that treating plants with a high concentration may induce indirect effects such as osmotic stress leading to the initiation of responses that are similar to defence responses. It has been shown that hyperosmotic stress leads to a fast calcium response in 
Arabidopsis (Stephan et al., 2016). Furthermore, MAPK6 and MAPK3 are activated in response to hyperosmotic stress while MAPK6, MAPK3 and MAPK4 are phosphorylated upon hypoosmotic stress (Droillard et al., 2002; Droillard et al., 2004; Colcombet and Hirt, 2008). A comparison of genes regulated upon $B$. cinerea infection and several abiotic stresses revealed that about $40-50 \%$ of the genes that were differentially regulated upon infection with $B$. cinerea were also subject to transcriptional reprogramming upon osmotic stress (Sham et al., 2015). To test whether xylohexaose triggers immune responses, the induction of defence responses upon xylohexaose treatment could be repeated with a higher carbohydrate concentration that is still in a physiological relevant range. As xylohexaose induced the activation of MAPK slightly, it seems likely that this carbohydrate also activated further immune responses upon treatment with a higher concentration. Xylohexaose is comprised of a chain of six $\beta-1,4$-linked unsubstituted xylose monomers and might be generated upon degradation of the hemicellulose class of xylans. The degradation of xylan involves mainly two enzyme classes: endo- $\beta-1,4-x y l a n a s e s$ and $\beta$-xylosidases (Polizeli et al., 2005).

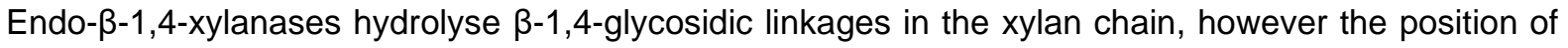
the bonds selected for hydrolysis depends on the chain length, presence of side chains and degree of branching (Polizeli et al., 2005; Kubicek et al., 2014). During the hydrolysis, xylooligosaccharides of varying length including xylohexaose are generated but also smaller products e.g. xylobiose are produced (Polizeli et al., 2005; Gonçalves et al., 2012; da Silva et al., 2015). The majority of fungal xylanases have been categorized to GH10 and GH11 (Kubicek et al., 2014) and can be found in plant pathogenic fungi including M. oryzae, V. dahliae and Fusarium oxysporum (Zhao et al., 2014). Furthermore, xylanases were shown to play a role in virulence. Simultaneous knockdown of ten xylanase encoding genes of $M$. oryzae resulted in a reduced penetration rate and a reduction in lesion size on barley (Nguyen et al., 2011). This supports the idea that plant arabinoxylan is degraded during the infection process by certain fungi leading to the release of xylooligosaccharides. Subsequently, xylohexaose or other xylooligosaccharides might be perceived by the plant as DAMP.

\subsubsection{Treatment with linear $\beta-1,3-g l u c a n$ oligosaccharides triggers MAPK phosphorylation}

Previously, unsubstituted linear $\beta$-1,3-glucans were shown to induce immune responses in tobacco (Klarzynski et al., 2000). In marked contrast, experiments conducted in this study in A. thaliana revealed that $\beta-1,3$-glucans of a length of five or six oligosaccharides were only able to slightly activate MAPK but not the influx of $\mathrm{Ca}^{2+}$ ions and the generation of ROS (Figure S14, Figure S15). As the induction of MAPK was only slight and the other tested responses were not induced, $\beta$-1,3-glucans were excluded from further analysis. However, a study from 2018 claimed that $\beta-1,3$,glucan hexamers and pentamers induce an influx in $\mathrm{Ca}^{2+}$ ions, activation of MAPK and induction of defence gene expression in a CERK1-dependent manner in Arabidopsis (Mélida et al., 2018). The discrepancies in the obtained results might be explained with the concentration that was used in the assays. Whilst 10 to $100 \mu \mathrm{g} \mathrm{ml}^{-1}$ of the $\beta$-1,3-glucan oligosaccharides were used in this study, 
$250 \mathrm{\mu g} \mathrm{ml}^{-1}$ of the respective substrates were used in the publication of Antonio Molina's research group (Mélida et al., 2018). $\beta$-1,3-glucans are the most abundant $\beta$-glucan in the fungal cell wall (Fesel and Zuccaro, 2016). Upon pathogen attack, chitinases as well as $\beta-1,3$-glucanase are secreted by the plant to hydrolyse fungal cell wall components and release elicitor-active oligosaccharides (Keen and Yoshikawa, 1983; Chisholm et al., 2006; Balasubramanian et al., 2012). Previous studies have shown that $\beta-1,3$-glucanases from soybean decompose fungal cell walls which result in the release of elicitor active $\beta-1,3$-oligosaccharides (Keen and Yoshikawa, 1983; Ham, 1991). Furthermore, a number of $\beta-1,3$-glucanases of Arabidopsis were shown to be transcriptionally up-regulated upon pathogen attack and proposed to function in pathogen defence (Dong et al., 1991; Uknes et al., 1992; Doxey et al., 2007). Similarly to soybean $\beta-1,3$-glucanases, $\beta-1,3$-glucanases from Arabidopsis could be involved in the degradation of $\beta-1,3$-glucan in the fungal cell wall and thereby generating $\beta$-1,3-glucan oligosaccharides. However, $\beta$-1,3-glucans are also present in plants and can be found in callose-rich papillae that are generated in close proximity to fungal penetration sites or are deposited at plasmodesmata for conductivity control and in the cell plate during cytokinesis (Chen and Kim, 2009; Underwood, 2012). Previously, it has also been suggested that $\beta$-1,3-glucan oligosaccharides might originate from plant papillae (Klarzynski et al., 2000). Thus, $\beta-1,3-$ glucan oligosaccharides might represent a MAMP and/or DAMP.

\subsubsection{MLGs act as DAMP and/or MAMP in barley}

MLG is an unbranched homopolymer consisting of glucose that are interconnected via both $\beta-1,3-$ and $\beta-1,4-g l y c o s i d i c ~ l i n k a g e s$ (Pauly et al., 2013). The experimental data generated in this study demonstrated that MLG oligosaccharides consisting of three or four glucose monomers from Megazyme elicit the generation of ROS and the activation of MAPK in barley (Figure 10). This indicates that these MLG derivatives can be perceived by barley and trigger the induction of immune responses and that there is no required minimal length for the eliciting activity. Additionally, MLG tri- and tetrasaccharides from a second carbohydrate supplier resulted in the generation of ROS and activation of MAPK (Figure 13) which verified the previous results. HPAEC-PAD and MALDI-TOF analysis of the MLG oligosaccharides revealed no major carbohydrate contamination (Figure 16, Figure 17) supporting the manufacturer's claim of high purity of a minimum of $95 \%$ and virtually excluding that possible contaminations in the preparation might be the causal elicitor. To elicit immune responses, MLGs were used at a concentration of $10 \mathrm{\mu g} \mathrm{ml}^{-1}$ (for MAPK assays) or at $100 \mathrm{\mu g} \mathrm{ml}^{-1}$ (for ROS burst assays). The used concentrations are in accordance or lower compared to concentrations used to trigger chitin-induced immune responses in barley (Scheler et al., 2016).

MLGs are found in the cell walls of most members of the Poales including the crop plants barley and oat (Burton and Fincher, 2009). In young barley plants, MLGs can be found e.g. in the epidermal and mesophyll cells in first leaves (Trethewey and Harris, 2002). Barley MLGs consist predominantly of $\beta$-1,4-linked cellotriosyl or cellotetrasyl units connected via $\beta$-1,3-linkages that are randomly distributed along the chain. The MLG oligosaccharides obtained from Megazyme were generated via 
enzymatic hydrolysis of the barley MLG polymer (Megazyme, Compton, Ireland). Thus, the MLG oligosaccharides exhibit the same structure as the MLG oligosaccharides found in barley. This together with the fact that the MLG oligosaccharides are derived from barley support the idea that MLG oligosaccharides act as DAMP in this crop plant. MLG oligosaccharides might be generated from the MLG polymer present in the cell wall of monocots during the infection process by microbial $\beta-1,3 ; 1,4-$ glucanases. The first fungal $\beta-1,3 ; 1,4$-glucanases have been identified and characterized in the anaerobic fungal strain Orpinomyces sp. PC-2 (Chen et al., 1997) and the aerobic fungus Talaromyces emersonii (Murray et al., 2001). Additionally, three extracellular $\beta-1,3 ; 1,4$-glucanases were identified in the plant pathogenic fungus Cochliobolus carbonum and shown to act on the MLG polymer of barley (Görlach et al., 1998). Enzymes that catalyze the hydrolysis of $\beta-1,3 ; 1,4-$ glucans can be found in the GH families 5, 6, 7, 8, 9, 11, 12, 16, 17, 26 and 51 (Carbohydrate Active Enzymes database, http://www.cazy.org/, Lombard et al., 2014). Plant pathogenic fungi that infect monocot species e.g. U. maydis, Fusarium graminerarum and M. oryzae were shown to harbour genes encoding for CWDEs that can be categorized in GH5, GH7, GH8, GH12 or GH16 (Zhao et al., 2014). Some of these enzymes may be secreted during the infection and exhibit $\beta-1,3 ; 1,4$-glucanase activity to degrade the $\beta-1,3 ; 1,4$-polymer present in the monocot cell wall. The activity of these enzymes might lead to the release of MLG oligosaccharides.

Interestingly, in the past years, MLGs have also been found in lichen as well as in fungal and bacterial species suggesting that they are more widespread than previously thought. For example, MLGs are present in thalli of the two lichens Cetaria islandica and Evernia prunastri (Honegger and Haisch, 2001). Also, MLGs are abundant cell wall components in the hyphal cell wall of the ascomycete A. fumigatus, a human pathogen that causes Aspergillus infections in immunocompromised patients (Samar et al., 2015). Notably, the inner walls of hyphae from the plant pathogen R. commune were also shown to contain MLGs. Structural analysis revealed that the $R$. commune MLG polymer consisted of the MLG tetrasaccharide G4G4G3G, the trisaccharide G4G3G and the dimer laminaribiose (Pettolino et al., 2009). The structure of the MLG oligosaccharides found in R. commune resembles the structures of the MLG oligosaccharides used in this study suggesting that MLGs oligosaccharides might be released from the fungal cell wall through the activity of extracellular $\beta-1,3 ; 1,4-$ glucanases. The perception of the released MLG oligosaccharides leads subsequently to the activation of defence responses. So far, two genes encoding for $\beta-1,3 ; 1,4$-glucanases have been identified in barley and the corresponding proteins, $H v G l b l$ and $H v G l b l l$, have been characterized. Both enzymes were active on the barley $\beta-1,3 ; 1,4$-polymer resulting in the release of tri-, tetra- and penta-saccharides (Woodward and Fincher, 1982). Transcripts of HvGlbl can be detected in young leaves correlating with $\beta-1,3 ; 1,4-$ glucanase activity found in the leaf extracts (Slakeski and Fincher, 1992). Similarly, $\beta-1,3 ; 1,4$-glucanases of the monocot crop plant wheat are present in young developing leaves (Roulin and Feller, 2001). Although the function and the exact localization of these proteins are not known, it was proposed that the identified $\beta-1,3 ; 1,4$-glucanases rather play a role in development than in immunity (Slakeski and Fincher, 1992; Roulin and Feller, 2001). This suggests that the characterized $\beta-1,3 ; 1,4$-glucanases are not implicated in the generation of MLG oligosaccharides from the fungal cell wall. However, further genes encoding for $\beta-1,3 ; 1,4$-polymer 
degrading enzymes could be present in the barley or wheat genome that play a role in fungal cell wall degradation.

Taken together, the data suggest that barley evolved the ability to perceive MLG oligosaccharides which results in the activation of immune responses. MLGs could originate either from barley or from a pathogen and therefore, might act as DAMP or MAMP or both.

\subsubsection{MLGs act as a MAMP in Arabidopsis}

It was demonstrated here that MLG oligosaccharides from two different carbohydrate suppliers were able to induce the influx of $\mathrm{Ca}^{2+}$ ions, the activation of MAPK and expression of the defence genes WRKY33 and WRKY53 in Arabidopsis (Figure 11, Figure 12, Figure 14, Figure 15). In contrast to barley, the generation of ROS could not be elicited robustly by the different MLG oligosaccharides in Arabidopsis (Figure $11 \mathrm{~B}$, Figure $14 \mathrm{~B}$ ). The luminol-based ROS burst assay is a comparatively variable and insensitive method and to obtain a reliable read-out, a high elicitor concentration has to be used. The concentration used to elicit a ROS burst might not be sufficient to induce the generation of ROS in Arabidopsis in a detectable amount while it might be sufficient to induce the ROS in barley. Nevertheless, the data generated in this study clearly demonstrate that Arabidopsis can perceive MLGs and that MLG recognition results in the activation of defence responses. No major carbohydrate contaminations could be identified during the analysis of the MLG oligosaccharides via HPAEC-PAD and MALDI-TOF which further support the conclusion that MLGs act as elicitors (Figure 16, Figure 17). The immune responses were elicited with MLG concentrations of $10 \mu \mathrm{g} \mathrm{ml}^{-1}$ or $100 \mathrm{~m} \mathrm{~m} \mathrm{ml}^{-1}$ which is in accordance or even lower to the amounts used to trigger chitin, peptidoglycan or $\beta$-1,3-glucan induced immune responses (Miya et al., 2007; Gust et al., 2007; Mélida et al., 2018). To further verify the eliciting activity of MLG oligosaccharides in Arabidopsis, MLG oligosaccharides of varying lengths were generated by hydrolyzing the barley $\beta-1,3 ; 1,4$-polymer with the $B$. subtilis lichenase. The hydrolysis products induced a fast calcium response, phosphorylation of MAPK6 and MAPK3 and expression of the defence genes WRKY33 and WRKY53 (Figure 19, Figure 20, Figure 21), providing further support that MLGs act as elicitors of immune responses in Arabidopsis.

MLGs are present in the cell walls of monocots and evolutionarily older plant lineages such as brown algae (Salmeán et al., 2017), liverworts (Popper and Fry, 2003) and Equisetum spp. (Fry et al., 2008; Sørensen et al., 2008), but are absent from Arabidopsis leaves (Zablackis et al., 1995). Thus, MLGs cannot act as DAMP in Arabidopsis. In the fungal kingdom, MLGs have been identified in the human pathogen A. fumigatus as well as in the plant pathogenic fungus $R$. commune (Pettolino et al., 2009; Samar et al., 2015). Furthermore, MLGs have been identified as exopolysaccharide of the bacterial species S. meliloti, which is an endosymbiont of Medicago sativa. The exopolysaccharide was shown to be important for root attachment but not nodulation (Pérez-Mendoza et al., 2015). The structure of the $\beta-1,3 ; 1,4$-polymer differs between the fungal and bacterial species. While the fungal MLG polymer consists of cellotriose units connected via $\beta$-1,3-linkages with only small amounts of cellotetraose units, the bacterial MLG consists of repeating units of $\beta-1,3$ and $\beta-1,4$ linkages (Pettolino et al., 2009; 
Pérez-Mendoza et al., 2015). Only the structure of the MLG trisaccharide used in this study resembles the structure of the bacterial MLG, while the structure of the MLG trisaccharide, MLG tetrasaccharide and longer MLG oligosaccharides generated during hydrolysis of the barley $\beta-1,3 ; 1,4-$ polymer is identical to the MLG structure found in $R$. commune. This suggests that MLG oligosaccharides might act as fungal MAMP in Arabidopsis. As only MLG oligosaccharides but not the $\beta-1,3 ; 1,4-$ polymer induced immune responses (Figure 19, Figure 20, Figure 21), MLG oligosaccharides of variable length have to be released from the polymer likely through the action of $\beta-1,3 ; 1,4-$ glucanases. During plant-pathogen interactions, genes encoding for chitinases as well as their activity increase. Chitinases hydrolyse chitin present in the fungal cell wall, which results in the generation of chitin oligomers that can be perceived by the plant (Pusztahelyi, 2018). Thus, it is conceivable to postulate that plants may also secrete $\beta-1,3 ; 1,4$-glucanases which act on fungal MLG polymers. Glucanases that hydrolyse MLGs are found in GH family 5, 6, 7, 8, 9, 11, 12, 16, 17, 26 and 51. The Arabidopsis genome harbours 13, 26, 33, 51 and 2 gene sequences encoding for enzymes in GH5, GH9, GH16, GH17 and GH51, respectively (Carbohydrate Active Enzymes database, http://www.cazy.org/, Lombard et al., 2014). Of these 125 proteins only two GH5 proteins, three GH9 proteins, twelve GH16 proteins, three enzymes categorized as $\mathrm{GH} 17$ and one protein designated as GH51 have been characterized (Carbohydrate Active Enzymes database, http://www.cazy.org/, Lombard et al., 2014). None of these proteins exhibit $\beta-1,3 ; 1,4-g l u c a n a s e$ activity, however, it might still be possible that not yet characterized enzymes belonging to these Arabidopsis GH families are able to hydrolyse fungal MLG polymers and thus are involved in the generation of MLG oligosaccharide MAMPs.

\subsubsection{The MLG tetrasaccharide elicits stronger responses than the MLG trisaccharide in Arabidopsis}

In this study, MLGs oligosaccharides were shown to robustly induce immune responses in Arabidopsis similar to those of well-characterized MAMPs e.g. fungal chitin and bacterial flg22 (Figure 11, Figure 12, Figure 14, Figure 15, Figure 19, Figure 20, Figure 21). The MLG tetramer as well as the MLG trimer were able to induce immune responses indicating that there is not a minimum length of MLGs required for activity. However, comparing the intensities of the $\mathrm{Ca}^{2+}$ influx, activation of MAPK6 and MAPK3 as well as defence gene expression induced by either the MLG tetrasaccharide or the MLG trisaccharide in Arabidopsis, it became evident that the tetramer triggered stronger responses than the trimer (Figure $11 \mathrm{~A}$ and $\mathrm{C}$, Figure 12, Figure $14 \mathrm{~A}$ and $\mathrm{C}$, Figure 15). The observation that longer oligosaccharides may have a higher MAMP activity is in line with previous studies for the carbohydrate MAMP chitin and the cell-wall derived DAMP OGs. In addition to polymeric chitin, chitin oligomers were shown to function as MAMPs. However, chitin tri- and tetramers were less effective in triggering immune responses than the chitin pentamer or longer chitin oligosaccharides (Petutschnig et al., 2010). Furthermore, treatment of Arabidopsis with trimeric OGs lead to activation of MAPK as well as expression of defence genes, but these responses were less 
pronounced than the responses induced by a mixture of long OGs (Davidsson et al., 2017). One explanation for the weaker responses upon trimer treatment could be that the putative MLG receptor has a higher binding affinity for the MLG tetrasaccharide than for the trimer. It was previously shown that phosphorylation of CERK1 is required for the induction of immune responses. Chitin oligomers that were able to trigger CERK1 phosphorylation comparable to the phosphorylation induced by polymeric chitin were also able to activate the generation of ROS and activation of MAPK, while chitin oligomers that induced less phosphorylation did not trigger immune responses (Petutschnig et al., 2010). A similar scenario might be possible for MLG perception. It might be possible that upon MLG perception, the respective receptor or the co-receptor are phosphorylated. The phosphorylation upon binding of the MLG trimer or the MLG tetramer might lead to an effective phosphorylation, albeit it might be less pronounced upon MLG trimer perception and thus resulting in a weaker response compared to the MLG tetramer. Furthermore, it could also be possible that due to a lower binding affinity of the receptor to the MLG trimer less receptor molecules are phosphorylated resulting in a weaker response. Previously, a dimerization model or "sandwich-like" dimerization for CERK1 and OsCEBiP was proposed in which one chitin oligosaccharide is bound to two CERK1 or two OsCEBiP molecules leading to the crosslinking of the two receptor molecules (Liu et al., 2012b; Hayafune et al., 2014). However, the formation of a stable complex required ligands of a minimum length of seven residues, while shorter chains lead to the formation of less-stable complexes (Liu et al., 2012b; Hayafune et al., 2014). Similarly, the putative MLG receptor may form a dimer upon cross-ligand binding. The complex may be formed upon MLG tetramer and MLG trimer perception but may be less stable upon treatment with the MLG trimer resulting in a reduced response in comparison to the MLG tetramer. To summarize, the data for chitin perception and signaling suggest that dimerization and phosphorylation are important for the induction and strength of the triggered immune responses and depend on the length of the elicitor. Similarly, these factors might also be important determinants for the amplitude and timing of MLG-induced responses. However, it remains to be shown whether these factors are determinants of the induction and amplitude of MLG triggered responses.

\subsubsection{The amplitude and timing of MLG-triggered responses in Arabidopsis differs from chitin- and flg22-induced responses}

\subsubsection{The calcium peak upon MLG elicitation occurs faster compared to chitin- and flg22-triggered calcium responses}

MLG treatment resulted in a rapid and transient calcium response. The shape of the MLG-induced $\mathrm{Ca}^{2+}$ signature was similar to the calcium response upon flg22 or chitin treatment but the peak occurred faster. Additionally, the calcium response induced by the MLG tri- and tetrasaccharide was less pronounced in comparison to flg22 and chitin, while the calcium spike in response to longer MLG oligosaccharides was higher than the chitin-induced calcium influx (Figure $11 \mathrm{~A}$, Figure $14 \mathrm{~A}$, Figure 19). This observation may indicate that either the concentration of MLG tri- or tetrasaccharides was not sufficient to induce the maximum calcium response or that longer MLG oligosaccharides are more 
efficient elicitors than shorter MLG oligosaccharides. This together with the finding that the timing of the MLG-induced calcium response is different from the chitin- and flg22-induced calcium spike supports the idea that the nature and efficiency of a stimulus perceived by the cell determines the calcium response including e.g. the amplitude, duration and frequency (Aldon et al., 2018). Although different MAMPs induce similar responses, these responses differ qualitatively and quantitatively. It has been proposed that the amplitude as well as the timing of the calcium response determine the outcome of MAMP treatment, although the exact mechanism is currently still unknown (Seybold et al., 2014). It is therefore possible that the amplitude and kinetic differences of calcium signatures might result in qualitative and quantitative differences between distinct immune responses such as ROS generation or transcriptional reprogramming triggered by MLGs and the MAMPs chitin and flg22.

\subsubsection{MLG perception leads to activation of MAPK6 and MAPK3}

Notably, treatment of Arabidopsis with MLG oligosaccharides resulted in the activation of MAPK6 and MAPK3 but not in the phosphorylation of MAPK4 (Figure $11 \mathrm{C}$, Figure $14 \mathrm{C}$, Figure 20). This is in contrast to results obtained for the MAMPs flg22 and chitin which trigger the activation of MAPK6, MAPK3 and MAPK4 (Figure $11 \mathrm{C}$, Figure $14 \mathrm{C}$, Figure 20). Plant MAPK cascades contribute to plant immune signaling and consist of three layers including a MAP kinase kinase kinase (MAP3K, MAPKKK or MEKK), a MAPK kinase kinase (MAP2K or MKK) and a MAP kinase (MAPK) (Rasmussen et al., 2012). Two cascades involved in plant immunity have been identified and well-characterized in Arabidopsis: 1) MAPKKK3/5-MKK4/5-MAPK3/6 and 2) MEKK1-MKK1/2-MPK4 (Devendrakumar et al., 2018). MAPK3, MAPK6 and MAPK4 exhibit different roles in plant defence responses. MAPK3 and MAPK6 are implicated in stomatal immunity and the biosynthesis of the secondary metabolites indole glucosinolates which upon enzymatic degradation releases biologically active compounds (Piasecka et al., 2015; Devendrakumar et al., 2018). Furthermore, biosynthesis of the phytohormone ethylene as well as of the antimicrobial compound camalexin is activated by MAPK3 and MAPK6 through the transcription factor WRKY33 (Rasmussen et al., 2012; Devendrakumar et al., 2018). MAPK4 is implicated in positive as well as negative regulation of flg22-responsive genes (Frei dit Frey et al., 2014; Li et al., 2015). Additionally, MAPK4 is also involved in jasmonic acid-mediated resistance as MAPK4 has a negative impact on salicylic acid accumulation (Petersen et al., 2000; Brodersen et al., 2006; Berriri et al., 2012). The observation that MAPK4 is not phosphorylated upon MLG perception might indicate that genes regulated by MAPK4 are not affected and therefore not involved in response to MLGs. To test whether the transcriptional reprogramming differs following MLG, flg22 or chitin perception, RNAseq analysis or microarray experiments could be performed on Arabidopsis seedlings treated with MLG oligosaccharides, chitin or flg22. 


\subsubsection{Activation of MAPKs and upregulation of WRKY33 and WRKY53 was less induced upon MLG treatment than upon chitin or flg22 elicitation}

This study showed that in comparison to MAPK activation and transcriptional upregulation in response to the MAMPs chitin and flg22, the activation of MAPK as well as the up-regulation of the transcription of the two defence genes WRKY33 and WRKY53 was less induced upon MLG perception (Figure 11 A, Figure 12, Figure 14 C, Figure 15, Figure 20, Figure 21). One explanation could be that the MLG oligosaccharide concentration was not sufficient to induce the maximum induction of gene transcription and MAPK phosphorylation. Alternatively, MLGs might be less efficient MAMPs compared to chitin and flg22. Previous studies have shown that a high number of genes are commonly upregulated upon MAMP treatment by flg22, elf18 or fungal chitin, however, the timing as well as the amplitude of the upregulation was different depending on the MAMP (Zipfel et al., 2006; Gust et al., 2007; Wan et al., 2008a; Li et al., 2016). The transcription of defence genes is controlled by MAPK activation and $\mathrm{Ca}^{2+}$-binding transcription factors that perceive changes in the intracellular $\mathrm{Ca}^{2+}$ concentration (Seybold et al., 2014). Thus, the lower transcriptional upregulation of WRKY33 and WRKY53 upon MLG treatment could be the result of the different timing and amplitude of the calcium response and MAPK activation, reinforcing the scenario already described above. More specifically, the activity as well as the transcription of WRKY33 is regulated through MAPK3 and MAPK6. The transcription factor WRKY33 is phosphorylated and thereby activated by MAPK3 and MAPK6. Phosphorylated WRKY33 can induce the expression of camalexin biosynthesis genes but also of itself by binding to its own promoter (Mao et al., 2011). The reduced phosphorylation status of MAPK6 and MAPK3 upon MLG perception might in turn result in a reduced number of phosphorylated WRKY33 and therefore in a lower transcript abundance of WRKY33 upon MLG treatment in comparison to transcript levels upon chitin or flg22 treatment. Furthermore, WRKY33 is found in a complex with MAPK4 and the MAPK4 substrate 1 (MKS1). Upon MAPK4 phosphorylation, WRKY33 is released and can be phosphorylated by MAPK3 and MAPK6 (Andreasson et al., 2005; Qiu et al., 2008). MLG oligosaccharide perception did not result in a detectable phosphorylation of MAPK4 while chitin and flg22 treatment induced activation of MAPK4 (Figure $11 \mathrm{C}$, Figure $14 \mathrm{C}$, Figure 20). Thus, no or only a low number of WRKY33 molecules might be released from the complex resulting in a lower induction of WRKY33 upon recognition of MLGs in comparison to chitin or flg22.

In conclusion, the data from the literature indicate that the calcium signature as well as the activation of specific MAPKs and the respective downstream components are important determinants of the transcriptional reprogramming. A valuable approach to test whether the transcriptional reprogramming differs after MLG oligosaccharide, chitin or flg22 treatment, RNAseq analysis of seedlings upon elicitor treatment could be performed. 


\subsubsection{MLG oligosaccharides treatment does not influence seedling growth}

The seedling growth inhibition represents a defence response that occurs within days and was previously observed for seedlings growing in the presence of flg22 or elf18 (Gómez-Gómez and Boller, 2000; Zipfel et al., 2006; Boller and Felix, 2009). To test whether MLG oligosaccharide treatment inhibits seedling growth, 5 day old seedlings were grown for 8 days in medium containing MLG oligosaccharides. However, the growth of Arabidopsis seedlings in presence of MLG oligosaccharides was not affected (Figure 22). This finding is in contrast to previous studies. The proteinaceous MAMPs flg22 and elf18 as well as the carbohydrate DAMP OGs were shown to inhibit seedling growth (Gómez-Gómez and Boller, 2000; Zipfel et al., 2006; Davidsson et al., 2017). On the opposite chitin dimers, trimers and tetramers as well as cellobiose were shown to promote seedling growth (Winkler et al., 2017; de Azevedo Souza et al., 2017). Possible explanations for this outcome could be that either the used MLG concentration was not sufficient to affect seedling growth positively or negatively and/or that the MLG oligosaccharides are not as efficient MAMPs. This result indicates that MLG oligosaccharides have no impact on seedling growth.

\subsubsection{Reverse genetics analyses reveal that so far unknown molecular components govern MLG perception in Arabidopsis}

The fast initiation of defence responses to MLG oligosaccharides suggests that MLG oligosaccharides are perceived at the cell surface as observed for chitin, flg22 and elf18. To identify molecular components involved in MLG perception, a reverse genetic screen was conducted in this study. Therefore, the MAPK activation upon MLG perception was analysed in multiple potentially involved MAMP/DAMP receptor and signaling mutants. The absence or reduction of MAPK phosphorylation in a mutant would indicate the involvement of the respective component in MLG perception. LysM-domain containing RLKs and RLPs are involved in the recognition of oligosaccharide MAMPs including ß-1,3-glucan (Miya et al., 2007; Petutschnig et al., 2010; Willmann et al., 2011; Mélida et al., 2018) and represent potential components of the MLG perception system. However, MLG induced MAPK activation in cerk1-2, lyk5-2 lyk4-1, lym2-1, lym2-4, lyk2-1 and lyt1-1 was as strong as in Col-0 indicating that these LysM-RLKs and LysM-RLPs are not required for MLG signaling (Figure 23). Furthermore, the LRR-RLKs FLS2 and EFR as well as the two co-receptor LRR-RLKs BAK1 and SOBIR1 were tested for their involvement in MLG perception. The MAPK activation upon MLG treatment in the respective mutants was neither compromised nor delayed compared to Col-0 suggesting that the tested LRR-RLKs are dispensable for MLG perception (Figure 24, Figure 25). Taken together, these data show that receptors and co-receptors that were already described to be involved in PTI signaling are not required for MLG perception. This suggests that MLG perception involves yet to be identified components. The Arabidopsis genome encodes for over $600 \mathrm{RLKs}$ and RLPs that might function as PRR or co-receptor in plant immunity, however, the ligands for only a few of these potential PRRs have been identified (Shiu and Bleecker, 2003; Macho and Zipfel, 2014). 
Thus, it is very likely that yet uncharacterized RLKs and RLPs mediate MLG perception and subsequent signaling.

Additionally, a forward genetic screen was performed to identify MAMP-sensitive and MAMP-insensitive $A$. thaliana ecotypes which could be used to identify molecular components involved in MLG perception and subsequent signal transduction. MAPK phosphorylation was observed in all 127 tested accessions suggesting that these ecotypes harbour the signaling machinery required to perceive MLGs and induction of immune responses (Figure 26, Figure S21, Figure S22, Figure S23). It also indicates that the required molecular components are evolutionary conserved among the tested accessions.

\subsubsection{Conclusion}

In the second part of this study, novel cell-wall derived DAMPs were identified by testing the ability of multiple poly- and oligosaccharides to induce immune responses. In agreement with studies published during the course of this project, cellohexaose as well as $\beta-1,3-$ glucan oligosaccharides were shown to act as elicitors of immune responses. Interestingly, MLG oligosaccharide treatment induced immune responses in the dicot model plant Arabidopsis and the monocot barley similar to defence responses triggered by other MAMPs or DAMPs. MLGs are not abundant cell wall components in Arabidopsis but are present in monocot grasses, including barley, as well as the fungal plant pathogen $R$. commune, suggesting that MLGs function in a plant species-specific manner as DAMP or MAMP (or both). So far, neither a reverse genetic screen nor a forward genetic screen revealed molecular components required for MLG perception and signal transduction. This suggests that novel components that have not been identified yet are required for MLG perception and signaling. The involvement of new components might explain the differences in the amplitude and timing of MLG-induced and chitin- or flg22-induced responses.

\subsubsection{Outlook}

It was demonstrated in this study that over 100 different Arabidopsis accessions and barley perceive MLGs leading to the subsequent activation of immune responses. In future studies, more monocot species such as Brachypodium distachyon or $O$. sativa and dicot species such as Medicago truncatula or poplar could be tested for their ability to perceive MLG oligosaccharides or the $\beta-1,3 ; 1,4$-polymer. Furthermore, it could be tested whether immune responses are induced in evolutionary older plant lineages such as mosses e.g. Marchantia polymorpha or Physcomitrella patens, horsetails e.g. Equisetum spp. or ferns e.g. Ceratopteris richardii upon MLG elicitation. This could answer the question whether the MLG perception system is evolutionary conserved or whether it evolved independently in several plant species. 
The identification and characterization of RLKs and RLPs involved in MLG signaling in Arabidopsis represents another very interesting subject for further analysis. The RLCK BIK1 is implicated in immune signaling as BIK1 interacts with FLS2, EFR, CERK1 and PEPRs (Tang et al., 2017). Thus, it might be possible that BIK1 does play a role in MLG signaling. To address the involvement of BIK1 in MLG signalling, MLG-triggered MAPK activation could be analysed in BIK1 mutants. Alternatively, a second forward genetic screen could be conducted. Therefore, wild-type Col-0 or Col-0 aequorin seeds could be mutagenized and screened for mutant plants that are impaired in MLG-induced MAPK activation or $\mathrm{Ca}^{2+}$ response, respectively. Mutant plants that are insensitive to MLGs can be screened for mutations in potential components required for MLG perception. This strategy has already been successfully exploited in the past to identify the FLS2 gene (Gómez-Gómez and Boller, 2000).

Analysis of the cell walls of the two ascomycete fungi $A$. fumigatus and $R$. commune revealed the presence of MLGs. Furthermore, mycelial morphology of three plant pathogenic fungi was deconstructed upon treatment with a bacterial $\beta-1,3 ; 1,4$-glucanase suggesting that they may contain MLGs (Xu et al., 2016). These data indicate that MLGs might be present in the cell wall of further fungi which would support the hypothesis that MLGs act as MAMPs. To address whether MLGs are abundant cell wall components of other fungi, the cell wall composition of further plant-pathogenic fungi could be analysed by immunohistological and enzymatic analysis. A $\beta-1,3 ; 1,4$-glucan specific antibody could be used to label $\beta-1,3 ; 1,4$-glucans that might be present in the tested fungal cell walls. Furthermore, cell wall preparations could be prepared from the respective fungi and be subjected to enzymatic hydrolysis with a lichenase. The resulting hydrolysates could be tested via TLC and/or HPAEC-PAD for the presence of MLG oligosaccharides.

So far, it has not been elucidated how elicitor active MLG oligosaccharides might be generated from the fungal cell wall. In soybean, the release of $\beta$-1,3-glucan oligosaccharides has been shown to be facilitated by the action of $\beta$-1,3-glucanases present in the secretome of soybean (Keen and Yoshikawa, 1983). Similarly, the secretome of Arabidopsis and barley could be analysed for the presence of $\beta-1,3 ; 1,4$-glucanases. The abundance of $\beta-1,3 ; 1,4$-glucanases in the secretome represents a first hint that MLG oligosaccharides might be generated upon hydrolysis of the fungal cell wall by plant of $\beta-1,3 ; 1,4$-glucanases. Additionally, the potentially identified $\beta-1,3 ; 1,4$-glucanases could be functionally characterized to verify their mode of action.

Overall, the proposed future analysis would provide novel insights into generation and perception of MLG oligosaccharides and might reveal yet unidentified molecular components involved in plant immunity. Furthermore, such analyses may reveal that MLGs are abundant cell wall components in more fungal species than previously thought. 


\section{References}

Albert, I., Böhm, H., Albert, M., Feiler, C.E., Imkampe, J., Wallmeroth, N., Brancato, C., Raaymakers, T.M., Oome, S., Zhang, H., Krol, E., Grefen, C., Gust, A.A., Chai, J., Hedrich, R., Van den Ackerveken, G. and Nürnberger, T. (2015) An RLP23-SOBIR-BAK1 complex mediates NLP-triggered immunity. Nature Plants, 1, 15140.

Aldon, D., Mbengue, M., Mazars, C. and Galaud, J.P. (2018) Calcium signalling in plant biotic interactions. Int. J. Mol. Sci., 19, 1-19.

Andreasson, E., Jenkins, T., Brodersen, P., Thorgrimsen, S., Petersen, N.H.T., Zhu, S., Qiu, J.-L., Micheelsen, P., Rocher, A., Petersen, M., Newman, M.-A., Nielsen, H.B., Hirt, H., Somssich, I., Mattson, O. and Mundy, J. (2005) The MAP kinase substrate MKS1 is a regulator of plant defense responses. EMBO J., 24, 2579-2589.

Antolín-Llovera, M., Petutsching, E.K., Ried, M.K., Lipka, V., Nürnberger, T., Robatzek, S. and Parniske, M. (2014) Knowing your friends and foes - plant receptor-like kinases as initiators of symbiosis or defence. New Phytol., 204, 791-802.

Ao, Y., Li, Z., Feng, D., Xiong, J.L., Liu, J., Li, J.-F., Wang, M., Wang, J., Liu, B. and Wang, H..B. (2014) OsCERK1 and OsRLCK176 play important roles in peptidoglycan and chitin signaling in rice innate immunity. Plant J., 80, 1072-1084.

Ardèvol, A. and Rovira, C. (2015) Reaction Mechanisms in Carbohydrate-Active Enzymes: Glycoside Hydrolases and Glycosyltransferases. Insights from ab Initio Quantum Mechanics/Molecular Mechanics Dynamic Simulations. J. Am. Chem. Soc., 137, 7528-7547.

Aspeborg, H., Coutinho, P.M., Wang, Y., Brumer, H. and Henrissat, B. (2012) Evolution, substrate specificity and subfamily classification of glycoside hydrolase family 5 (GH5). BMC Evol. Biol., 12, 186. Available at: BMC Evolutionary Biology.

Atmodjo, M.A., Hao, Z. and Mohnen, D. (2013) Evolving Views of Pectin Biosynthesis. Annu. Rev. Plant Biol., 64, 747-779.

Azevedo Souza, C. de, Li, S., Lin, A.Z., Boutrot, F., Grossmann, G., Zipfel, C. and Somerville, S.C. (2017) Cellulose-derived oligomers act as damage-associated molecular patterns and trigger defense-like responses. Plant Physiol., 173, 2383-2398.

Aziz, A., Poinssot, B., Daire, X., Adrian, M., Bézier, A., Lambert, B., Joubert, J.M. and Pugin, A. (2003) Laminarin Elicits Defense Responses in Grapevine and Induces Protection Against Botrytis cinerea and Plasmopara viticola. Mol. Plant-Microbe Interact., 16, 1118-1128.

Bacete, L., Mélida, H., Miedes, E. and Molina, A. (2018) Plant cell wall-mediated immunity: cell wall changes trigger disease resistance responses. Plant J., 93, 614-636.

Balasubramanian, V., Vashisht, D., Cletus, J. and Sakthivel, N. (2012) Plant $\beta-1,3-g l u c a n a s e s:$ Their biological functions and transgenic expression against phytopathogenic fungi. Biotechnol. Lett., 34, 1983-1990.

Baldrian, P. and Valášková, V. (2008) Degradation of cellulose by basidiomycetous fungi. FEMS Microbiol. Rev., 32, 501-521.

Bartels, S. and Boller, T. (2015) Quo vadis, Pep? Plant elicitor peptides at the crossroads of immunity, stress, and development. J. Exp. Bot., 66, 5183-5193.

Bartels, S., Lori, M., Mbengue, M., Verk, M. Van, Klauser, D., Hander, T., Böni, R., Robatzek, S. and Boller, T. (2013) The family of peps and their precursors in Arabidopsis: Differential expression and localization but similar induction of pattern-Triggered immune responses. J. Exp. 
Bot., 64, 5309-5321.

Bellincampi, D., Cervone, F. and Lionetti, V. (2014) Plant cell wall dynamics and wall-related susceptibility in plant-pathogen interactions. Front. Plant Sci., 5, 1-8. Available at: http://journal.frontiersin.org/article/10.3389/fpls.2014.00228/abstract.

Berriri, S., Garcia, A.V., dit Frey, N.F., Rozhon, W., Pateyron, S., Leonhardt, N., Montillet, J.-L., Leung, J., Hirt, H. and Colcombet, J. (2012) Constitutively active mitogen-activated protein kinase versions reveal functions of Arabidopsis MPK4 in pathogen defense signaling. Plant Cell, 24, 4281-4293.

Bi, G., Liebrand, T.W.H., Cordewener, J.H.G., America, A.H.P., Xu, X. and Joosten, M.H.A.J. (2014) Arabidopsis thaliana receptor-like protein At RLP23 associates with the receptor-like kinase At SOBIR1. Plant Signaling and Behavior, 9:e27937.

Bigeard, J., Colcombet, J. and Hirt, H. (2015) Signaling mechanisms in pattern-triggered immunity (PTI). Mol. Plant, 8, 521-539. Available at: http://dx.doi.org/10.1016/j.molp.2014.12.022.

Block, A., Guangyong, L., Fu, Z.Q., and Alfano, J.R. (2008) Phytopathogen type III effector weaponry and their plant targets. Curr Opin Plant Biol., 11(4), 369-403.

Boller, T. and Felix, G. (2009) A Renaissance of Elicitors: Perception of Microbe-Associated Molecular Patterns and Danger Signals by Pattern-Recognition Receptors. Annu. Rev. Plant Biol., 60, 379-406.

Bradford, M.M., (1976) A rapid and sensitive method for the quantification of microgram quantities of protein utilizing the principle of protein-dye binding. Analytical Biochemistry, 72, 248-254.

Bretthauer, R.K. and Castellino, F.J. (1999) Glycosylation of Pichia pastoris-derived proteins. Biotechnol. Appl. Biochem., 30, 193-200.

Brink, J. Van Den and Vries, R.P. De (2011) Fungal enzyme sets for plant polysaccharide degradation. Appl. Microbiol. Biotechnol., 91, 1477-1492.

Brodersen, P., Petersen, M., Nielsen, H.B., Zhu, S., Newman, M.A., Shokat, K.M., Rietz, S., Parker, J. and Mundy, J. (2006) Arabidopsis MAP kinase 4 regulates salicylic acid- and jasmonic acid/ethylene-dependent responses via EDS1 and PAD4. Plant J., 47, 532-546.

Brutus, A., Sicilia, F., Macone, A., Cervone, F. and Lorenzo, G. De (2010) A domain swap approach reveals a role of the plant wall-associated kinase 1 (WAK1) as a receptor of oligogalacturonides. Proc. Natl. Acad. Sci. U. S. A., 107, 9452-9457.

Burton, R.A., Wilson, S.M., Hrmova, M., Harvey, A.J., Shirley, N.J., Medhurst, A., Stone, B.A., Newbigin, E.J., Bacic, A. and Fincher, G.B. (2006) Cellulose Synthase - Like CsIF Genes Mediate Synthesis of Cell Wall (13;1,4)- $\beta$-D-glucans. Science, 311(5769), 1940-1942.

Burton, R.A. and Fincher, G.B. (2009) $(1,3 ; 1,4)-\beta$-D-glucans in cell walls of the poaceae, lower plants, and fungi: A tale of two linkages. Mol. Plant, 2, 873-882. Available at: http://dx.doi.org/10.1093/mp/ssp063.

Burton, R.A. and Fincher, G.B. (2014) Evolution and development of cell walls in cereal grains. Front. Plant Sci., 5, 1-15.

Burton, R.A., Gidley, M.J. and Fincher, G.B. (2010) Heterogeneity in the chemistry, structure and function of plant cell walls. Nat. Chem. Biol., 6, 724-732. Available at: http://dx.doi.org/10.1038/nchembio.439.

Cabrera, J.C., Boland, A., Messiaen, J., Cambier, P. and Cutsem, P. Van (2008) Egg box conformation of oligogalacturonides: The time-dependent stabilization of the elicitor-active 
conformation increases its biological activity. Glycobiology, 18, 473-482.

Caffall, K.H. and Mohnen, D. (2009) The structure, function, and biosynthesis of plant cell wall pectic polysaccharides. Carbohydr. Res., 344, 1879-1900. Available at: http://dx.doi.org/10.1016/j.carres.2009.05.021.

Cao, Y., Liang, Y., Tanaka, K., Nguyen, C.T., Jedrzejczak, R.P., Joachimiak, A. and Stacey, G. (2014) The kinase LYK5 is a major chitin receptor in Arabidopsis and forms a chitin-induced complex with related kinase CERK1. Elife, 3:e03766.

Chang, X., Xu, B., Bai, Y., Luo, H., Ma, R., Shi, P. and Yao, B. (2017) Role of N-linked glycosylation in the enzymatic properties of a thermophilic GH 10 xylanase from Aspergillus fumigatus expressed in Pichia pastoris. PLoS One, 12, 1-13.

Chatterjee, S., Chaudhury, S., McShan, A.C., Kaur, K., and De Guzman, R.N. (2013) Structure and Biophysics of Type III Secretion in Bacteria. Biochemistry, 52(15), 2508-2517.

Chen, H., Li, X.L. and Ljungdahl, L.G. (1997) Sequencing of a 1,3-1,4- $\beta$-D-glucanase (lichenase) from the anaerobic fungus Orpinomyces strain PC-2: Properties of the enzyme expressed in Escherichia coli and evidence that the gene has a bacterial origin. J. Bacteriol., 179, 6028-6034.

Chen, X.Y. and Kim, J.Y. (2009) Callose synthesis in higher plants. Plant Signal. Behav., 4, 489492.

Chinchilla, D., Bauer, Z., Regenass, M., Boller, T. and Felix, G. (2006) The Arabidopsis receptor kinase FLS2 binds flg22 and determines the specificity of flagellin perception. Plant Cell, 18, 465-476.

Chinchilla, D., Zipfel, C., Robatzek, S., Kemmerling, B., Nürnberger, T., Jones, J.D.G., Felix, G. and Boller, T. (2007) A flagellin-induced complex of the receptor FLS2 and BAK1 initiates plant defence. Nature, 448, 497-500.

Chisholm, S.T., Coaker, G., Day, B. and Staskawicz, B.J. (2006) Host-microbe interactions: Shaping the evolution of the plant immune response. Cell, 124, 803-814.

Chomczynski, P. (1993) A reagent for the single-step simultaneous isolation of RNA, DNA and proteins from cell and tissue samples. Biotechniques, 15, 532-537.

Chowdhury, J., Henderson, M., Schweizer, P., Burton, R.A., Fincher, G.B. and Little, A. (2014) Differential accumulation of callose, arabinoxylan and cellulose in nonpenetrated versus penetrated papillae on leaves of barley infected with Blumeria graminis f. sp. hordei. New Phytol., 204, 650-660.

Christov, L.P. and Prior, B.A. (1993) Esterases of xylan-degrading microorganisms: Production, properties, and significance. Enzyme Microb. Technol., 15, 460-475.

Claverie, J., Balacey, S., Lemaître-Guillier, C., Brulé, D., Chiltz, A., Granet, L., Noirot, E., Daire, X., Darblade, B., Héloir, M.-C. and Poinssot, B. (2018) The cell wall-derived xyloglucan is a new DAMP triggering plant immunity in vitis vinifera and arabidopsis thaliana. Front. Plant Sci., 871, 1-14.

Colcombet, J. and Hirt, H. (2008) Arabidopsis MAPKs: A complex signalling network involved in multiple biological processes. Biochem. J., 413, 217-226.

Corradini, C., Cavazza, A. and Bignardi, C. (2012) High-Performance Anion-Exchange Chromatography Coupled with Pulsed Electrochemical Detection as a Powerful Tool to Evaluate Carbohydrates of Food Interest: Principles and Applications. Int. J. Carbohydr. Chem., 2012, 113. 
Cuskin, F., Lowe, E.C., Temple, M.J., Zhu, Y., Cameron, E.A., Pudlo, N.A., Porter, N.T., Urs, K., Thompson, A.J., Cartmell, A., Rogowski, A., Hamilton, B.S., Chen, R., Tolbert, T.J., Piens, K., Bracke, D., Vervecken, W., Hakki, Z., Speciale, G., Munoz-Munoz, J.L., Day, A., Pena, M.J., McLean, R., Suits, M.D., Boraston, A.B., Atherly, T., Ziemer, C.J., Williams, S.J., Davies, G.J., Abbott, D.W., Martens, E.C. and Gilbert, H.J. (2015) Human gut Bacteroidetes can utilize yeast mannan through a selfish mechanism. Nature, 517, 165-169.

Daly, R. and Hearn, M.T.W. (2005) Expression of heterologous proteins in Pichia pastoris: A useful experimental tool in protein engineenring and production. J. Mol. Recognit., 18, 119-138.

Dangl, J.L., Horvath, D.M. and Staskawicz, B.J. (2013) Pivoting the Plant Immune System. Science., 341, 745-751.

Davidsson, P., Broberg, M., Kariola, T., Sipari, N., Pirhonen, M. and Palva, E.T. (2017) Short oligogalacturonides induce pathogen resistance-associated gene expression in Arabidopsis thaliana. BMC Plant Biol., 17, 1-17. Available at: http://dx.doi.org/10.1186/s12870-016-0959-1.

Davies, G. and Henrissat, B. (1995) Structures and mechanisms of glycosyl hydrolases. Structure, 3, 853-859.

Davies, G.J., Wilson, K.S. and Henrissat, B. (1997) Nomenclature for sugar-binding subsites in glycosyl hydrolases [1]. Biochem. J., 321, 557-559.

Decreux, A. and Messiaen, J. (2005) Wall-associated kinase WAK1 interacts with cell wall pectins in a calcium-induced conformation. Plant Cell Physiol., 46, 268-278.

Denoux, C., Galletti, R., Mammarella, N., Gopalan, S., Werck, D., Lorenzo, G. De, Ferrari, S., Ausubel, F.M. and Dewdney, J. (2008) Activation of defense response pathways by OGs and Flg22 elicitors in Arabidopsis seedlings. Mol. Plant, 1, 423-445.

Devendrakumar, K.T., Li, X. and Zhang, Y. (2018) MAP kinase signalling: interplays between plant PAMP- and effector-triggered immunity. Cell. Mol. Life Sci., 75, 1-9. Available at: http://link.springer.com/10.1007/s00018-018-2839-3.

Doblin, M.S., Pettolino, F.A., Wilson, S.M., Campbell, R., Burton, R.A., Fincher, G.B., Newbigin, E. and Bacic, A. (2009) A barley cellulose synthase-like CSLH gene mediates $(1,3 ; 1,4)-\beta-D-$ glucan synthesis in transgenic Arabidopsis. Proc. Natl. Acad. Sci. U. S. A., 106, 5996-6001.

Dodds, P.N. and Rathjen, J.P. (2010) Plant immunity: towards an integrated view of plant-pathogen interactions. Nat. Rev. Genet., 11, 539-548. Available at: http://www.nature.com/doifinder/10.1038/nrg2812.

Dong, X., Mindrinos, M., Davis, K.R. and Ausubel, F.M. (1991) Induction of Arabidopsis defense genes by virulent and avirulent Pseudomonas syringae strains and by a cloned avirulence gene. Plant Cell, 3, 61-72.

Doxey, A.C., Yaish, M.W.F., Moffatt, B.A., Griffith, M. and McConkey, B.J. (2007) Functional divergence in the Arabidopsis $\beta-1,3$-glucanase gene family inferred by phylogenetic reconstruction of expression states. Mol. Biol. Evol., 24, 1045-1055.

Droillard, M.J., Boudsocq, M., Barbier-Brygoo, H. and Laurière, C. (2002) Different protein kinase families are activated by osmotic stresses in Arabidopsis thaliana cell suspensions: Involvement of the MAP kinases AtMPK3 and AtMPK6. FEBS Lett., 527, 43-50.

Droillard, M.J., Boudsocq, M., Barbier-Brygoo, H. and Laurière, C. (2004) Involvement of MPK4 in osmotic stress response pathways in cell suspensions and plantlets of Arabidopsis thaliana: Activation by hypoosmolarity and negative role in hyperosmolarity tolerance. FEBS Lett., $\mathbf{5 7 4}$, 42-48. 
Eisenthal, R., Danson, M.J. and Hough, D.W. (2007) Catalytic efficiency and kcat/KM: a useful comparator? Trends Biotechnol., 25, 247-249.

Faulkner, C., Petutschnig, E., Benitez-Alfonso, Y., Beck, M., Robatzek, S., Lipka, V. and Maule, A.J. (2013) LYM2-dependent chitin perception limits molecular flux via plasmodesmata. Proc. Natl. Acad. Sci. U. S. A., 110, 9166-9170.

Felle, H.H. (2006) Apoplastic pH during low-oxygen stress in barley. Ann. Bot., 98, 1085-1093.

Ferrari, S., Galletti, R., Denoux, C., Lorenzo, G. De, Ausubel, F.M. and Dewdney, J. (2007) Resistance to Botrytis cinerea induced in Arabidopsis by elicitors is independent of salicylic acid, ethylene, or jasmonate signaling but requires PHYTOALEXIN DEFICIENT3. Plant Physiol., 144, 367-379.

Ferrari, S., Savatin, D. V., Sicilia, F., Gramegna, G., Cervone, F. and Lorenzo, G. De (2013) Oligogalacturonides: Plant damage-associated molecular patterns and regulators of growth and development. Front. Plant Sci., 4, 1-9.

Fesel, P.H. and Zuccaro, A. (2016) $\beta$-glucan: Crucial component of the fungal cell wall and elusive MAMP in plants. Fungal Genet. Biol., 90, 53-60. Available at: http://dx.doi.org/10.1016/j.fgb.2015.12.004.

Frei dit Frey, N., Garcia, A.V., Bigeard, J., et al. (2014) Functional analysis of Arabidopsis immunerelated MAPKs uncovers a role for MPK3 as negative regulator of inducible defences. Genome Biol., 15, 1-22.

Fry, S.C., Nesselrode, B.H.W.A., Miller, J.G. and Mewburn, B.R. (2008) Mixed-linkage $(1 \rightarrow 3,1 \rightarrow 4)$ $\beta$-D-glucan is a major hemicellulose of Equisetum (horsetail) cell walls. New Phytol., 179, 104115.

Galletti, R., Denoux, C., Gambetta, S., Dewdney, J., Ausubel, F.M., Lorenzo, G. De and Ferrari, S. (2008) The AtrbohD-mediated oxidative burst elicited by oligogalacturonides in Arabidopsis is dispensable for the activation of defense responses effective against Botrytis cinerea. Plant Physiol., 148, 1695-1706.

Galletti, R., Ferrari, S. and Lorenzo, G. de (2011) Arabidopsis MPK3 and MPK6 play different roles in basal and oligogalacturonide- or flagellin-induced resistance against Botrytis cinerea. Plant Physiol., 157, 804-814.

Garron, M.L. and Cygler, M. (2014) Uronic polysaccharide degrading enzymes. Curr. Opin. Struct. Biol., 28, 87-95. Available at: http://dx.doi.org/10.1016/j.sbi.2014.07.012.

Gastebois, A., Mouyna, I., Simenel, C., Clavaud, C., Coddeville, B., Delepierre, M., Latgé, J.P. and Fontaine, T. (2010) Characterization of a new $\beta(1-3)$-glucan branching activity of Aspergillus fumigatus. J. Biol. Chem., 285, 2386-2396.

Gibson, D.G., Young, L., Chuang, R.Y., Venter, J.C., Hutchison, C.A. and Smith, H.O. (2009) Enzymatic assembly of DNA molecules up to several hundred kilobases. Nat. Methods, 6, 343345.

Gimenez-lbanez, S., Ntoukakis, V. and Rathjen, J.P. (2009) The LysM receptor kinase CERK1 mediates bacterial perception in Arabidopsis. Plant Signal. Behav., 4, 539-541.

Glass, N.L., Schmoll, M., Cate, J.H.D. and Coradetti, S. (2013) Plant Cell Wall Deconstruction by Ascomycete Fungi. Annu. Rev. Microbiol., 67, 477-498.

Gomes, A.R., Byregowda, S.M., Veeregowda, B.M. and Balamurugan, V. (2016) An Overview of Heterologous Expression Host Systems for the Production of Recombinant Proteins. Adv. Anim. Vet. Sci., 4, 346-356. 
Gómez-Gómez, E., Ruíz-Roldán, M.C., Pietro, A. Di, Roncero, M.I.G. and Hera, C. (2002) Role in pathogenesis of two endo- $\beta-1,4-x y l a n a s e ~ g e n e s$ from the vascular wilt fungus Fusarium oxysporum. Fungal Genet. Biol., 35, 213-222.

Gómez-Gómez, L. and Boller, T. (2000) FLS2: An LRR receptor-like kinase involved in the perception of the bacterial elicitor flagellin in Arabidopsis. Mol. Cell, 5, 1003-1011.

Gómez-Gómez, L., Felix, G. and Boller, T. (1999) A single locus determines sensitivity to bacterial flagellin in Arabidopsis thaliana. Plant J., 18, 277-284.

Gonçalves, T.A., Damásio, A.R.L., Segato, F., Alvarez, T.M., Bragatto, J., Brenelli, L.B., Citadini, A.P.S., Murakami, M.T., Roller, R., Paes Leme, A.F., Prade, R.A. and Squina, F.M. (2012) Functional characterization and synergic action of fungal xylanase and arabinofuranosidase for production of xylooligosaccharides. Bioresour. Technol., 119, 293-299.

Görlach, J.M., Knaap, E. Van Der and Walton, J.D. (1998) Cloning and targeted disruption of MLG1, a gene encoding two of three extracellular mixed-linked glucanases of Cochliobolus carbonum. Appl. Environ. Microbiol., 64, 385-391.

Grignon, C. and Sentenac, H. (1991) pH and ionic conditions in the apoplast. Annu. Rev. Plant Physiol. Plant Mol. Biol, 42, 103-128.

Grishutin, S., Gusakov, A.V., Dzedzyulya, E.I. and Sinitsyn, A.P. (2006) A lichenase-like family 12 endo-(1 $\rightarrow 4)-\beta$-glucanase from Aspergillus japonicus: study of the substrate specificity and mode of action on $\beta$-glucans in comparison with other glycoside hydrolases. Carbohydrate Research, 341, 218-229.

Gurkan, C. and Ellar, D.J. (2003) Expression in Pichia pastoris and purification of a membraneacting immunotoxin based on a synthetic gene coding for the Bacillus thuringiensis Cyt2Aa1 toxin. Protein Expr Purif, 29, 103-116.

Gust, A.A. (2015) Peptidoglycan Perception in Plants. PLoS Pathog., 11, 1-7.

Gust, A.A., Biswas, R., Lenz, H.D., Rauhut, T., Ranf, S., Kemmerling, B., Götz, F., Glawischnig, E., Lee, J., Felix, G. and Nürnberger, T. (2007) Bacteria-derived peptidoglycans constitute pathogen-associated molecular patterns triggering innate immunity in Arabidopsis. J. Biol. Chem., 282, 32338-32348.

Gust, A.A. and Felix, G. (2014) Receptor like proteins associate with SOBIR1-type of adaptors to form bimolecular receptor kinases. Curr. Opin. Plant Biol., 21, 104-111. Available at: http://dx.doi.org/10.1016/j.pbi.2014.07.007.

Hacquard, S., Kracher, B., Maekawa, T., Vernaldi, S., Schulze-Lefert, P. and Themaat, E.V.L. Van (2013) Mosaic genome structure of the barley powdery mildew pathogen and conservation of transcriptional programs in divergent hosts. Proc. Natl. Acad. Sci. U. S. A., 110, 2219-2228.

Ham, K.-S., Kauffmann, S., Albersheim, P. and Darvill, A.G. (1991) Host-Pathogen Interactions XXXIX. A Soybean Pathogenesis-Related Protein with $\beta-1,3-G l u c a n a s e$ Activity Releases Phytoalexin Elicitor-Active Heat-Stable Fragments from Fungal Walls. Mol. Plant-Microbe Interact., 4, 545.

Hann, D.R. and Rathjen, J.P. (2007) Early events in the pathogenicity of Pseudomonas syringae on Nicotiana benthamiana. Plant J., 49, 607-618.

Hayafune, M., Berisio, R., Marchetti, R., Silipo, A., Kayama, M., Desaki, Y., Arima, S., Sqegila, F., Ruggiero, A., Tokuyasu, K., Molinaro, A., Kaku, H. and Shibuya, N. (2014) Chitin-induced activation of immune signaling by the rice receptor CEBiP relies on a unique sandwich-type dimerization. Proc. Natl. Acad. Sci. U. S. A., 111. 
Heese, A., Hann, D.R., Gimenez-lbanez, S., Jones, A.M.E., He, K., Li, J., Schroeder, J.I., Peck, S.C. and Rathjen, J.P. (2007) The receptor-like kinase SERK3/BAK1 is a central regulator of innate immunity in plants. Proc. Natl. Acad. Sci. U. S. A., 104, 12217-12222.

Henrissat, B. (1991) A classification of glycosyl hydrolases based on amino acid sequence similarities. Biochem. J., 280, 309-316.

Herscovics, A. (2001) Structure and function of Class I a1,2-mannosidases involved in glycoprotein synthesis and endoplasmic reticulum quality control. Biochimie, 83, 757-762.

Honegger, R. and Haisch, A. (2001) Immunocytochemical localtion of the $(1 \rightarrow 3)(1 \rightarrow 4)-\beta$-glucan lichenin in the lichen-forming ascomycete Cetraria islandica (Icelandic moss). New Phytologist, 150, 739-746.

Hosseini, S. and Martinez-Chapa, S.O. (2017) Fundamentals of MALDI-ToF-MS Analysis,.

Houston, K., Tucker, M.R., Chowdhury, J., Shirley, N. and Little, A. (2016) The plant cell wall: A complex and dynamic structure as revealed by the responses of genes under stress conditions. Front. Plant Sci., 7, 1-18.

Hrmova, M. and Fincher, G.B. (1993) Purification and properties of three $(1 \rightarrow 3)-\beta$-D-glucanase isoenzymes from young leaves of barley (Hordeum vulgare). Biochem. J., 289, 453-461.

Hrmova, M., Garrett, T.P.J. and Fincher, G.B. (1995) Subsite affinities and disposition of catalytic amino acids in the substrate-binding region of barley $1,3-\beta$-glucanases. Implications in plantpathogen interactions. J. Biol. Chem., 270, 14556-14563.

Huecas, S., Villalba, M. and Rodríguez, R. (2001) Ole e 9, a major olive pollen allergen is a 1,3- $\beta$ glucanase: Isolation, characterization, amino acid sequence, and tissue specificity. J. Biol. Chem., 276, 27959-27966.

Huffaker, A., Pearce, G. and Ryan, C.A. (2006) An endogenous peptide signal in Arabidopsis activates components of the innate immune response. Proc. Natl. Acad. Sci. U. S. A., 103, 10098-10103.

Jacobs, A.K., Lipka, V., Burton, R.A., Panstruga, R., Strizhov, N., Schulze-lefert, P. and Fincher, G.F. (2003) An Arabidopsis Callose Synthase, GSL5, Is Required for Wound and Papillary Callose Formation, The Plant Cell., 15, 2503-2513.

Jehle, A.K., Fürst, U., Lipschis, M., Albert, M. and Felix, G. (2013a) Perception of the novel MAMP eMax from different Xanthomonas species requires the Arabidopsis receptor-like protein ReMAX and the receptor kinase SOBIR. Plant Signal. Behav., 8, 11-13.

Jehle, A.K., Lipschis, M., Albert, M., Fallahzadeh-Mamaghani, V., Fürst, U., Mueller, K. and Felix, G. (2013b) The receptor-like protein ReMAX of Arabidopsis detects the microbeassociated molecular pattern eMax from Xanthomonas. Plant Cell, 25, 2330-2340.

Jones, J.D.G. and Dangl, J.L. (2006) The plant immune system. Nature, 444, 323-329.

Kaku, H., Nishizawa, Y., Ishii-Minami, N., Akimoto-Tomiyama, C., Dohmae, N., Takio, K., Minami, E. and Shibuya, N. (2006) Plant cells recognize chitin fragments for defense signaling through a plasma membrane receptor. Proc. Natl. Acad. Sci. U. S. A., 103, 11086-11091.

Kamoun, S. (2006) A Catalogue of the Effector Secretome of Plant Pathogenic Oomycetes. Annu. Rev. Phytopathol., 44, 41-60.

Keegstra, K. (2010) Plant Cell Walls. Plant Physiol., 154, 483-486. 
Keen, N.T. and Yoshikawa, M. (1983) $\beta-1,3-E n d o g l u c a n a s e$ from Soybean Releases Elicitor-Active Carbohydrates from Fungus Cell Walls. Plant Physiol., 71, 460-465.

Kessel, A. and Ben-Tal, N. (2018). Enzyme Kinetics in Kessel, A. and Ben-Tal, N., eds. Introduction to Proteins: Structure, Function and Motion. Second Edition. Chapman and Hall/CRC.

King, B.C., Waxman, K.D., Nenni, N. V., Walker, L.P., Bergstrom, G.C. and Gibson, D.M. (2011) Arsenal of plant cell wall degrading enzymes reflects host preference among plant pathogenic fungi. Biotechnol. Biofuels, 4, 1-14.

Kishimoto, K., Kouzai, Y., Kaku, H., Shibuya, N., Minami, E. and Nishizawa, Y. (2010) Perception of the chitin oligosaccharides contributes to disease resistance to blast fungus Magnaporthe oryzae in rice. Plant J., 64, 343-354.

Klarzynski, O., Plesse, B., Joubert, J.M., Yvin, J.C., Kopp, M., Kloareg, B. and Fritig, B. (2000) Linear $\beta-1,3$ glucans are elicitors of defense responses in tobacco. Plant Physiol., 124, 10271037.

Koshland, D.E. (1953) Stereochemistry and the Mechanism of Enzymatic Reactions. Biological Reviews, 28, 416-436.

Kouzai, Y., Mochizuki, S., Nakajima, K., Desaki, Y., Hayafune, M., Miyazaki, H., Yokotani, N., Ozawa, K., Minami, E., Kaku, H., Shibuya, N. and Nishizawa, Y. (2014) Targeted gene disruption of OsCERK1 reveals its indispensable role in chitin perception and involvement in the peptidoglycan response and immunity in rice. Mol. Plant-Microbe Interact., 27, 975-982.

Kouzai, Y., Nakajima, K., Hayafune, M., Ozawa, K., Kaku, H., Shibuya, N., Minami, E. and Nishizawa, Y. (2014) CEBiP is the major chitin oligomer-binding protein in rice and plays a main role in the perception of chitin oligomers. Plant Mol. Biol., 84, 519-528.

Kover, P.X., Valdar, W., Trakalo, J., Scarcelli, N., Ehrenreich, I.M., Purugganan, M.D., Durrant, C. and Mott, R. (2009) A multiparent advanced generation inter-cross to fine-map quantitative traits in Arabidopsis thaliana. PLoS Genet., 5:e1000551.

Kubicek, C.P., Starr, T.L. and Glass, N.L. (2014) Plant Cell Wall-Degrading Enzymes and Their Secretion in Plant-Pathogenic Fungi. Annu. Rev. Phytopathol., 52, 427-451.

Kunze, G., Zipfel, C., Robatzek, S., Niehaus, K., Boller, T. and Felix, G. (2004) The N terminus of bacterial elongation factor Tu elicits innate immunity in Arabidopsis plants. Plant Cell, 16, 34963507.

Lagaert, S., Beliën, T. and Volckaert, G. (2009) Plant cell walls: Protecting the barrier from degradation by microbial enzymes. Semin. Cell Dev. Biol., 20, 1064-1073.

Li, B., Jiang, S., Yu, X., Cheng, C., Chen, S., Cheng, A., Yuan, J.S., Jiang, D., He, P. and Shan, L. (2015) Phosphorylation of trihelix transcriptional repressor ASR3 by MAP KINASE4 negatively regulates Arabidopsis immunity. Plant Cell, 27, 839-856.

Li, B., Meng, X., Shan, L. and He, P. (2016) Transcriptional Regulation of Pattern-Triggered Immunity in Plants. Cell Host Microbe, 19, 641-650.

Li, J., Wen, J., Lease, K.A., Doke, J.T., Tax, F.E. and Walker, J.C. (2002) BAK1, an Arabidopsis LRR receptor-like protein kinase, interacts with BRI1 and modulates brassinosteroid signaling. Cell, 110, 213-222.

Liang, X. and Zhou, J.-M. (2018) Receptor-Like Cytoplasmic Kinases: Central Players in Plant Receptor Kinase-Mediated Signaling. Annu. Rev. Plant Biol., 69, 267-299. 
Liebrand, T.W.H., Berg, G.C.M. Van Den, Zhang, Z., Smit, P., Cordewener, J.H.G., America, A.H.P., Sklenar, J., Jones, A.M.E., Tameling, W.I.L., Robatzek, S., Thomma, B.H.J. and Joosten, M.H.A.J. (2013) Receptor-like kinase SOBIR1/EVR interacts with receptor-like proteins in plant immunity against fungal infection. Proc. Natl. Acad. Sci. U. S. A., 110, 1001010015.

Liebrand, T.W.H., Burg, H.A. van den and Joosten, M.H.A.J. (2014) Two for all: Receptorassociated kinases SOBIR1 and BAK1. Trends Plant Sci., 19, 123-132. Available at: http://dx.doi.org/10.1016/j.tplants.2013.10.003.

Liu, B., Li, J.F., Ao, Y., Qu, J., Li, Z., Su, J., Zhang, Y., Liu, J., Feng, D., Qi, K., He, Y., Wang, J. and Wang, H.-B. (2012a) Lysin motif-containing proteins LYP4 and LYP6 play dual roles in peptidoglycan and chitin perception in rice innate immunity. Plant Cell, 24, 3406-3419.

Liu, T., Liu, Z., Song, C., Hu, Y., Han, Z., She, J., Fan, F., Wang, J., Jin, C., Chang, J., Zhou, J.-M. and Chai, J. (2012b) Chitin-induced dimerization activates a plant immune receptor. Science. 336, 1160-1164.

Liu, Z., Wu, Y., Yang, F., Zhang, Y., Chen, S., Xie, Q., Tian, X. and Zhou, J.M. (2013) BIK1 interacts with PEPRs to mediate ethylene-induced immunity. Proc. Natl. Acad. Sci. U. S. A., 110, $6205-6210$.

Lombard, V., Golaconda Ramulu, H., Drula, E., Coutinho, P.M. and Henrissat, B. (2014) The carbohydrate-active enzymes database (CAZy) in 2013. Nucleic Acids Res., 42, 490-495.

Lorenzo, G. De and Ferrari, S. (2002) Polygalacturonase-inhibiting proteins in defense against phytopathogenic fungi. Curr. Opin. Plant Biol., 5, 295-299.

Lovering, A.L., Safadi, S.S. and Strynadka, N.C.J. (2012) Structural Perspective of Peptidoglycan Biosynthesis and Assembly. Annu. Rev. Biochem., 81, 451-478.

Lu, D., Wu, S., Gao, X., Zhang, Y., Shan, L. and He, P. (2010) A receptor-like cytoplasmic kinase, BIK1, associates with a flagellin receptor complex to initiate plant innate immunity. Proc. Natl. Acad. Sci. U. S. A., 107, 496-501.

Macho, A.P. and Zipfel, C. (2014a) Plant PRRs and the activation of innate immune signaling. Mol. Cell, 54, 263-272. Available at: http://dx.doi.org/10.1016/j.molcel.2014.03.028.

Macho, A.P. and Zipfel, C. (2015) Targeting of plant pattern recognition receptor-triggered immunity by bacterial type-III secretion system effectors. Curr. Opin. Microbiol., 23, 14-22. Available at: http://dx.doi.org/10.1016/j.mib.2014.10.009.

Malinovsky, F.G., Fangel, J.U. and Willats, W.G.T. (2014) The role of the cell wall in plant immunity. Front. Plant Sci., 5, 1-12.

Mao, G., Meng, X., Liu, Y., Zheng, Z., Chen, Z. and Zhang, S. (2011) Phosphorylation of a WRKY transcription factor by two pathogen-responsive MAPKs drives phytoalexin biosynthesis in Arabidopsis. Plant Cell, 23, 1639-1653.

McFarlane, H.E., Döring, A. and Persson, S. (2014) The Cell Biology of Cellulose Synthesis. Annu. Rev. Plant Biol., 65, 69-94.

McGregor, N., Morar, M., Fenger, T.H., Stogios, P., Lenfant, N., Yin, V., Xu, X., Evdokimova, E., Cui, H., Henrissat, B., Savchenko, A. and Brumer, H. (2016) Structure-function analysis of a mixed-linkage $\beta$-glucanase/xyloglucanase from the key ruminal bacteroidetes prevotella bryantii B14. J. Biol. Chem., 291, 1175-1197.

Mélida, H., Sopeña-Torres, S., Bacete, L., Garrido-Arandia, M., Jordá, L., López, G., MuñozBarrios, A., Pacios, L.F. and Molina, A. (2018a) Non-branched $\beta$-1,3-glucan oligosaccharides trigger immune responses in Arabidopsis. Plant J., 93, 34-49. 
Michaelis, L. and Menten, M.L. (1913) Die Kinetik der Invertinwirkung. Biochem. Z. 49, 333-369

Michaud, P., Costa, A. Da, Courtois, B. and Courtois, J. (2003) Polysaccharide lyases: Recent developments as biotechnological tools. Crit. Rev. Biotechnol., 23, 233-266.

Miya, A., Albert, P., Shinya, T., et al. (2007) CERK1, a LysM receptor kinase, is essential for chitin elicitor signaling in Arabidopsis. Proc. Natl. Acad. Sci. U. S. A., 104, 19613-19618.

Monaghan, J. and Zipfel, C. (2012) Plant pattern recognition receptor complexes at the plasma membrane. Curr. Opin. Plant Biol., 15, 349-357. Available at: http://dx.doi.org/10.1016/j.pbi.2012.05.006.

Murray, P.G., Grassick, A., Laffey, C.D., Cuffe, M.M., Higgins, T., Savage, A. V., Planas, A. and Tuohy, M.G. (2001) Isolation and characterization of a thermostable endo- $\beta$-glucanase active on 1,3-1,4- $\beta$-D-glucans from the aerobic fungus Talaromyces emersonii CBS 814.70. Enzyme Microb. Technol., 29, 90-98.

Muzzarelli RAA (1977) Chitin, 1st ed. edn. Oxford ; New York: Pergamon Press.

Nakamura, A.M., Nascimento, A.S. and Polikarpov, I. (2017) Structural diversity of carbohydrate esterases. Biotechnol. Res. Innov., 1, 35-51. Available at: http://dx.doi.org/10.1016/j.biori.2017.02.001.

Nam, K.H. and Li, J. (2002) BRI1/BAK1, a receptor kinase pair mediating brassinosteroid signaling. Cell, 110, 203-212.

Nguyen, Q.B., Itoh, K., Vu, B. Van, Tosa, Y. and Nakayashiki, H. (2011) Simultaneous silencing of endo- $\beta-1,4$ xylanase genes reveals their roles in the virulence of Magnaporthe oryzae. Mol. Microbiol., 81, 1008-1019.

Nowara, D., Schweizer, P., Gay, A., Lacomme, C., Shaw, J., Ridout, C., Douchkov, D., Hensel, G. and Kumlehn, J. (2010) HIGS: Host-induced gene silencing in the obligate biotrophic fungal pathogen Blumeria graminis. Plant Cell, 22, 3130-3141.

Park, Y.B. and Cosgrove, D.J. (2015) Xyloglucan and its interactions with other components of the growing cell wall. Plant Cell Physiol., 56, 180-194.

Patova, O.A., Golovchenko, V. V. and Ovodov, Y.S. (2014) Pectic polysaccharides: Structure and properties. Russ. Chem. Bull., 63, 1901-1924.

Pauly, M., Gille, S., Liu, L., Mansoori, N., Souza, A. de, Schultink, A. and Xiong, G. (2013) Hemicellulose biosynthesis. Planta, 238, 627-642.

Pérez-Mendoza, D., Rodríguez-Carvajal, M.Á., Romero-Jiménez, L., Araujo Farias, G. De, Lloret, J., Gallegos, M.T. and Sanjuán, J. (2015) Novel mixed-linkage $\beta$-glucan activated by c-di-GMP in Sinorhizobium meliloti. Proc. Natl. Acad. Sci. U. S. A., 112, E757-E765.

Petersen, M., Brodersen, P., Naested, H., Andreasson, E., Lindhart, U., Johansen, B., Nielsen, H.B., Lacy, M., Austin, M.J., Parker, J.E., Sharma, S.B., Klessig, D.F., Martienssen, R., Mattson, O., Jensen, B. and Mundy, J. (2000) Arabidopsis MAP kinase 4 negatively regulates systemic acquired resistance. Cell, 103, 1111-1120.

Pettolino, F., Sasaki, I., Turbic, A., Wilson, S.M., Bacic, A., Hrmova, M. and Fincher, G.B. (2009) Hyphal cell walls from the plant pathogen Rhynchosporium secalis contain $(1,3 / 1,6)-\beta-D-$ glucans, galacto- and rhamnomannans, $(1,3 ; 1,4)-\beta-D-$ glucans and chitin. FEBS J., 276, 41224133.

Petutschnig, E.K., Jones, A.M.E., Serazetdinova, L., Lipka, U. and Lipka, V. (2010) The Lysin Motif Receptor-like Kinase (LysM-RLK) CERK1 is a major chitin-binding protein in Arabidopsis 
thaliana and subject to chitin-induced phosphorylation. J. Biol. Chem., 285, 28902-28911.

Peumans, W.J., Barre, A., Derycke, V., Rougé, P., Zhang, W., May, G.D., Delcour, J.A., Leuven, F. Van and Damme, E.J.M. Van (2000) Purification, characterization and structural analysis of an abundant $\beta-1,3-$ glucanase from banana fruit. Eur. J. Biochem., 267, 1188-1195.

Piasecka, A., Jedrzejczak-Rey, N. and Bednarek, P. (2015) Secondary metabolites in plant innate immunity: Conserved function of divergent chemicals. New Phytol., 206, 948-964.

Planas, A. (2000) Bacterial 1,3-1,4- $\beta$-glucanases: Structure, function and protein engineering. Biochim. Biophys. Acta - Protein Struct. Mol. Enzymol., 1543, 361-382.

Plummer, T.H., Elder, J.H., Alexander, S., Phelan, A.W. and Tarentino, A.L. (1984) Demonstration of peptide: $\mathrm{N}$-glycosidase $\mathrm{F}$ activity in endo- $\beta$ - $\mathrm{N}$-acetylglucosaminidase $\mathrm{F}$ preparations. J. Biol. Chem., 259, 10700-10704.

Polizeli, M.L.T.M., Rizzatti, A.C.S., Monti, R., Terenzi, H.F., Jorge, J.A. and Amorim, D.S. (2005) Xylanases from fungi: Properties and industrial applications. Appl. Microbiol. Biotechnol., 67, 577-591.

Popper, Z.A. and Fry, S.C. (2003) Primary cell wall composition of bryophytes and charophytes. Ann. Bot., 91, 1-12.

Postel, S. and Kemmerling, B. (2009) Plant systems for recognition of pathogen-associated molecular patterns. Semin. Cell Dev. Biol., 20, 1025-1031.

Pusztahelyi, T. (2018) Chitin and chitin-related compounds in plant-fungal interactions. Mycology, 9, 189-201. Available at: https://doi.org/10.1080/21501203.2018.1473299.

Qin, Z., Yan, Q., Lei, J., Yang, S., Jiang, Z. and Wu, S. (2015) The first crystal structure of a glycoside hydrolase family $17 \beta-1,3-$ glucanosyltransferase displays a unique catalytic cleft. Acta Crystallogr. Sect. D Biol. Crystallogr., 71, 1714-1724.

Qiu, J.L., Fiil, B.K., Petersen, K., Nielsen, H.B., Botanga, C.J., Thorgrimsen, S., Palma, K., Suarez-Rodriguez, M.C., Lichota, J., Brodersen, P., Grasser, K.D., Mattson, O., Glazebrook, J., Mundy, J. and Petersen, M. (2008) Arabidopsis MAP kinase 4 regulates gene expression through transcription factor release in the nucleus. EMBO J., 27, 2214-2221.

Ranf, S. Grimmer, J., Pöschl, Y., Pecher, P., Chinchilla, D., Scheel, D. and Lee, J. (2012) Defense-Related Calcium Signaling Mutants Uncovered via a Quantitative High-Throughput Screen in Arabidopsis thaliana. Molecular Plant, 5, 115-130.

Rasmussen, M.W., Roux, M., Petersen, M. and Mundy, J. (2012) MAP kinase cascades in Arabidopsis innate immunity. Front. Plant Sci., 3, 1-6.

Rayon, C., Lerouge, P. and Faye, L. (1998) The protein N-glycosylation in plants. J. Exp. Bot., 49, 1463-1472.

Rentel, M.C. and Knight, M.R. (2004) Oxidative stress-induced calcium signaling in Arabidopsis. Plant Physiol., 53, 287-299.

Robatzek, S., Bittel, P., Chinchilla, D., Köchner, P., Felix, G., Shiu, S.H. and Boller, T. (2007) Molecular identification and characterization of the tomato flagellin receptor LeFLS2, an orthologue of Arabidopsis FLS2 exhibiting characteristically different perception specificities. Plant Mol. Biol., 64, 539-547.

Robinson, P.K. (2015) Enzymes: principles and biotechnological applications. Essays Biochem., 59, $1-41$. 
Roulin, S. and Feller, U. (2001) Reversible accumulation of $(1 \rightarrow 3,1 \rightarrow 4)-\beta$-glucan endohydrolase in wheat leaves under sugar depletion. J. Exp. Bot., 52, 2323-2332.

Roux, M., Schwessinger, B., Albrecht, C., Chinchilla, D., Jones, A., Holton, N., Malinovsky, F.G., Tör, M., de Vries, S. and Zipfel, C. (2011) The Arabidopsis leucine-rich repeat receptor-like kinases BAK1/SERK3 and BKK1/SERK4 are required for innate immunity to hemibiotrophic and biotrophic pathogens. Plant Cell, 23, 2440-2455.

Salmeán, A.A., Duffieux, D., Harholt, J., Qin, F., Michel, G., Czjzek, M., Willats, W.G.T. and Hervé, C. (2017) Insoluble $(1 \rightarrow 3),(1 \rightarrow 4)$ - $\beta$-Dglucan is a component of cell walls in brown algae (Phaeophyceae) and is masked by alginates in tissues. Sci. Rep., 7, 1-11.

Samar, D., Kieler, J.B. and Klutts, J.S. (2015) Identification and deletion of Tft1, a predicted glycosyltransferase necessary for cell wall $\beta-1,3 ; 1,4$-glucan synthesis in Aspergillus fumigatus. PLoS One, 10, 1-14.

Schagerlöf, H., Nilsson, C., Gorton, L., Tjerneld, F., Stålbrand, H. and Cohen, A. (2009) Use of $18 \mathrm{O}$ water and ESI-MS detection in subsite characterisation and investigation of the hydrolytic action of an endoglucanase. Anal. Bioanal. Chem., 394, 1977-1984.

Scheler, B., Schnepf, V., Galgenmüller, C., Ranf, S. and Hückelhoven, R. (2016) Barley disease susceptibility factor RACB acts in epidermal cell polarity and positioning of the nucleus. J. Exp. Bot., 67, 3263-3275.

Scheller, H.V. and Ulvskov, P. (2010) Hemicelluloses. Annu. Rev. Plant Biol., 61, 263-289.

Schultink, A., Liu, L., Zhu, L. and Pauly, M. (2014) Structural diversity and function of xyloglucan sidechain substituents. Plants, 3, 526-542.

Schulze, B., Mentzel, T., Jehle, A.K., Mueller, K., Beeler, S., Boller, T., Felix, G. and Chinchilla, D. (2010) Rapid heteromerization and phosphorylation of ligand-activated plant transmembrane receptors and their associated kinase BAK1. J. Biol. Chem., 285, 9444-9451.

Schwessinger, B., Roux, M., Kadota, Y., Ntoukakis, V., Sklenar, J., Jones, A. and Zipfel, C. (2011) Phosphorylation-dependent differential regulation of plant growth, cell death, and innate immunity by the regulatory receptor-like kinase BAK1. PLoS Genet., 7.

Selin, C., Kievit, T.R. de, Belmonte, M.F. and Fernando, W.G.D. (2016) Elucidating the role of effectors in plant-fungal interactions: Progress and challenges. Front. Microbiol., 7, 1-21.

Seybold, H., Trempel, F., Ranf, S., Scheel, D., Romeis, T. and Lee, J. (2014) $\mathrm{Ca}^{2+}$ signalling in plant immune response: From pattern recognition receptors to $\mathrm{Ca}^{2+}$ decoding mechanisms. New Phytol., 204, 782-790.

Sham, A., Moustafa, K., Al-Ameri, S., Al-Azzawi, A., Iratni, R. and AbuQamar, S. (2015) Identification of Arabidopsis candidate genes in response to biotic and abiotic stresses using comparative microarrays. PLoS One, 10, 1-21.

Shimizu, T., Nakano, T., Takamizawa, D., et al. (2010) Two LysM receptor molecules, CEBiP and OsCERK1, cooperatively regulate chitin elicitor signaling in rice. Plant J., 64, 204-214.

Shinya, T., Motoyama, N., Ikeda, A., Wada, M., Kamiya, K., Hayafune, M., Kaku, H. and Shibuya, N. (2012) Functional characterization of CEBiP and CERK1 homologs in arabidopsis and rice reveals the presence of different chitin receptor systems in plants. Plant Cell Physiol., 53, 16961706.

Shinya, T., Nakagawa, T., Kaku, H. and Shibuya, N. (2015) Chitin-mediated plant-fungal interactions: Catching, hiding and handshaking. Curr. Opin. Plant Biol., 26, 64-71. Available at: http://dx.doi.org/10.1016/j.pbi.2015.05.032. 
Shiu, S.H. and Bleecker, A.B. (2003) Expansion of the receptor-like kinase/Pelle gene family and receptor-like proteins in Arabidopsis. Plant Physiol., 132, 530-543.

Shoseyov, O., Shani, Z. and Levy, I. (2006) Carbohydrate Binding Modules: Biochemical Properties and Novel Applications. Microbiol. Mol. Biol. Rev., 70, 283-295.

Silva, L.A. de O. da, Terrasan, C.R.F. and Carmona, E.C. (2015) Purification and characterization of xylanases from Trichoderma inhamatum. Electron. J. Biotechnol., 18, 307-313. Available at: http://dx.doi.org/10.1016/j.ejbt.2015.06.001.

Sinha, J., Plantz, B.A., Inan, M. and Meagher, M.M. (2005) Causes of proteolytic degradation of secreted recombinant proteins produced in methylotrophic yeast Pichia pastoris: Case study with recombinant ovine interferon-т. Biotechnol. Bioeng., 89, 102-112.

Slakeski, N. and Fincher, G.B. (1992) Developmental Regulation of (1->3,1->4)- $\beta$-D-Glucanase Gene Expression in Barley. Plant. Physiol., 99, 1226-1231.

Sørensen, I., Pettolino, F.A., Wilson, S.M., Doblin, M.S., Johansen, B., Bacic, A. and Willats, W.G.T. (2008) Mixed-linkage $(1 \rightarrow 3),(1 \rightarrow 4)-\beta$-D-glucan is not unique to the Poales and is an abundant component of Equisetum arvense cell walls. Plant J., 54, 510-521.

Spanu, P.D., Abbott, J.C., Amselem, J., et al. (2010) Genome expansion and gene loss in powdery mildew fungi reveal tradeoffs in extreme parasitism. Science., 330, 1543-1546.

Stephan, A.B., Kunz, H.H., Yang, E. and Schroeder, J.I. (2016) Rapid hyperosmotic-induced Ca ${ }^{2+}$ responses in Arabidopsis thaliana exhibit sensory potentiation and involvement of plastidial KEA transporters. Proc. Natl. Acad. Sci. U. S. A., 113, E5242-E5249.

Strasser, R. (2016) Plant protein glycosylation. Glycobiology, 26, 926-939.

Sundqvist, G., Stenvall, M., Berglund, H., Ottoson, J. and Brumer, H. (2007) A general and robust method for the quality control of intact proteins using LC-ESI-MS. Journal of Chromatography, 852, 188-194.

Takai, R., Isogai, A., Takayama, S. and Che, F.S. (2008) Analysis of flagellin perception mediated by flg22 receptor OsFLS2 in rice. Mol. Plant-Microbe Interact., 21, 1635-1642.

Tang, D., Wang, G. and Zhou, J.-M. (2017) Receptor Kinases in Plant-Pathogen Interactions: More Than Pattern Recognition. Plant Cell, 29, 618-637.

Tang, J., Han, Z., Sun, Y., Zhang, H., Gong, X. and Chai, J. (2015) Structural basis for recognition of an endogenous peptide by the plant receptor kinase PEPR1. Cell Res., 25, 110-120. Available at: http://dx.doi.org/10.1038/cr.2014.161.

Trethewey, J.A.K. and Harris, P.J. (2002) Location of $(1 \rightarrow 3)$ - and $(1 \rightarrow 3),(1 \rightarrow 4)-\beta$-D-glucans in vegetative cell walls of barley (Hordeum vulgare) using immunogold labelling. New Phytol., 154, 347-358.

Uknes, S., Mauch-Mani, B., Moyer, M., et al. (1992) Acquired resistance in arabidopsis. Plant Cell, 4, 645-656.

Underwood, W. (2012) The plant cell wall: A dynamic barrier against pathogen invasion. Front. Plant Sci., 3, 1-6.

Varrot, A., Schuelein, M., Fruchard, S., Driguez, H. and Davies, G.J. (2001) Atomic resolution structure of endoglucanase Cel5A in complex with methyl 4,4II,4III,4IV-tetrathio- $\alpha$ cellopentoside hihlights the alternative binding modes targeted by substrate mimics. Acta Crystallogr Sect D Biol Crystallogr, 57(11), 1739-1742. 
Viborg, A.H., Terrapon, N., Lombard, V., Michel, G., Czjzek, M., Henrissat, B. and Brumer, H. (2019) A subfamily roadmap for functional glycogenomics of the evolutionarily diverse Glycoside Hydrolase Family 16 (GH16). J. Biol. Chem., 16, jbc.RA119.010619.

Voigt, C.A. (2014) Callose-mediated resistance to pathogenic intruders in plant defense-related papillae. Front. Plant Sci., 5, 1-6.

Vuong, T. V. and Wilson, D.B. (2010) Glycoside hydrolases: Catalytic base/nucleophile diversity. Biotechnol. Bioeng., 107, 195-205.

Wan, J., Tanaka, K., Zhang, X.C., Son, G.H., Brechenmacher, L., Nguyen, T.H.N. and Stacey, G. (2012) LYK4, a lysin motif receptor-like kinase, is important for chitin signaling and plant innate immunity in Arabidopsis. Plant Physiol., 160, 396-406.

Wan, J., Zhang, X. and Stacey, G. (2008a) Chitin signaling and plant disease resistance. Mol. Plant Pathol., 6, 831-833.

Wan, J., Zhang, X.C., Neece, D., Ramonell, K.M., Clough, S., Kim, S.Y., Stacey, M.G. and Stacey, G. (2008b) A LysM receptor-like kinase plays a critical role in chitin signaling and fungal resistance in Arabidopsis. Plant Cell, 20, 471-481.

Wang, C., Wang, G., Zhang, C., Zhu, P., Dai, H., Yu, N., He, Z., Xu, L. and Wang, E. (2017) OsCERK1-Mediated Chitin Perception and Immune Signaling Requires Receptor-like Cytoplasmic Kinase 185 to Activate an MAPK Cascade in Rice. Mol. Plant, 10, 619-633. Available at: http://dx.doi.org/10.1016/j.molp.2017.01.006.

Werten, M.W.T., Bosch, T.J. van den, Wind, R.D., Mooibroek, H. and Wolf, F.A. de (1999) High-yield secretion of recombinant gelatins by Pichia pastoris. Yeast, 15, 1087-1096.

Willmann, R., Lajunen, H.M., Erbs, G., Newman, M.-A., Kolb, D., Tsuda, K., Katagiri, F., Fliegmann, J., Bono, J.-J., Cullimore, J.V., Jehle, A.K., Götz, F., Kulik, F., Molinaro, A., Lipka, V., Gust, A.A. and Nürnberger, T. (2011) Arabidopsis lysin-motif proteins LYM1 LYM3 CERK1 mediate bacterial peptidoglycan sensing and immunity to bacterial infection. Proc. Natl. Acad. Sci. U. S. A., 108, 19824-19829.

Winkler, A.J., Dominguez-Nuñez, J.A., Aranaz, I., Poza-Carrión, C., Ramonell, K., Somerville, S. and Berrocal-Lobo, M. (2017) Short-chain chitin oligomers: Promoters of plant growth. Mar. Drugs, 15, 1-21.

Wojtkowiak, A., Witek, K., Hennig, J. and Jaskolski, M. (2013) Structures of an active-site mutant of a plant 1,3-B-glucanase in complex with oligosaccharide products of hydrolysis. Acta Crystallogr. Sect. D Biol. Crystallogr., 69, 52-62.

Woodward, J.R. and Fincher, G.B. (1982) Substrate specificities and kinetic properties of two $(1 \rightarrow 3)$, (1 $\rightarrow 4)-\beta$-d-glucan endo-hydrolases from germinating barley (Hordeum vulgare). Carbohydr. Res., 106, 111-122.

Wu, S., Halley, J.E., Luttig, C., Fernekes, L.M., Darvill, A.G., Albersheim, P. and Barr, H. (2006) Gene Knockout Analysis , Purification, and Heterologous Expression. Society, 72, 986-993.

Xiang, T., Zong, N., Zou, Y., Wu, Y., Zhang, J., Xing, W., Li, Y., Tang, X., Zhu, L., Chai, J. and Zhou, J.-M. (2008) Pseudomonas syringae Effector AvrPto Blocks Innate Immunity by Targeting Receptor Kinases. Curr. Biol., 18, 74-80.

Xu, T., Zhu, T. and Li, S. (2016) $\beta$-1,3-1,4-glucanase gene from Bacillus vellezensis ZJ20 exerts antifungal effect on plant pathogenic fungi. World J Microbiol Biotechnol, 32(2):26.

Yamaguchi, K., Yamada, K., Ishikawa, K., Yoshimura, S., Hayashi, N., Uchihashi, K., Ishihama, N., Kishi-Kaboshi, M., Takahasi, A., Tsuge, S., Ochiai, H., Tada, Y., Shimamoto, K., Yoshioka, H. and Kawasaki, T. (2013) A receptor-like cytoplasmic kinase targeted by a plant 
pathogen effector is directly phosphorylated by the chitin receptor and mediates rice immunity. Cell Host Microbe, 13, 347-357. Available at: http://dx.doi.org/10.1016/j.chom.2013.02.007.

Yamaguchi, Y., Pearce, G. and Ryan, C.A. (2006) The cell surface leucine-rich repeat receptor for AtPep1, an endogenous peptide elicitor in Arabidopsis, is functional in transgenic tobacco cells. Proc. Natl. Acad. Sci. U. S. A., 103, 10104-10109.

Yin, Y., Mao, X., Yang, J., Chen, X., Mao, F. and Xu, Y. (2012) DbCAN: A web resource for automated carbohydrate-active enzyme annotation. Nucleic Acids Res., 40, 445-451.

Yu, X., Feng, B., He, P. and Shan, L. (2017) From Chaos to Harmony: Responses and Signaling upon Microbial Pattern Recognition. Annu. Rev. Phytopathol., 55, 109-137.

Zablackis, E., Huang, J., Müller, B., Darvill, A.G. and Albersheim, P. (1995) Characterization of the cell-wall polysaccharides of Arabidopsis thaliana leaves. Plant Physiol., 107, 1129-1138.

Zang, H., Xie, S., Zhu, B., Yang, X., Gu, C., Hu, B., Gao, T., Chen, Y. and Gao, X. (2019) Mannan oligosaccharides trigger multiple defence responses in rice and tobacco as a novel danger-associated molecular pattern. Mol. Plant Pathol., 20, 1067-1079.

Zhang, J., Li, W., Xiang, T., et al. (2010) Receptor-like cytoplasmic kinases integrate signaling from multiple plant immune receptors and are targeted by a Pseudomonas syringae effector. Cell Host Microbe, 7, 290-301. Available at: http://dx.doi.org/10.1016/j.chom.2010.03.007.

Zhang, J., Shao, F., Li, Y., Cui, H., Chen, L., Li, H., Zou, Y., Long, C., Lan, L., Chai, J., Chen, S., Tang, X. and Zhou, J.-M. (2007) A Pseudomonas syringae Effector Inactivates MAPKs to Suppress PAMP-Induced Immunity in Plants. Cell Host Microbe, 1, 175-185.

Zhang, L., Kars, I., Essenstam, B., Liebrand, T.W.H., Wagemakers, L., Elberse, J., Tagkalki, P., Tjoitang, D., van den Acjerceken, G. and van Kan, J.A.L. (2014) Fungal endopolygalacturonases are recognized as microbe-associated molecular patterns by the arabidopsis receptor-like protein RESPONSIVENESS TO BOTRYTIS POLYGALACTURONASES. Plant Physiol., 164, 352-364.

Zhang, W., Fraiture, M., Kolb, D., Löffelhardt, B., Desaki, Y., Boutrot, F.F.G., Tör, M., Zipfel, C., Gust, A.A. and Brunner, F. (2013) Arabidopsis RECEPTOR-LIKE PROTEIN30 and receptorlike kinase SUPPRESSOR OF BIR1-1/EVERSHED mediate innate immunity to necrotrophic fungi. Plant Cell, 25, 4227-4241.

Zhang, X.C., Wu, X., Findley, S., Wan, J., Libault, M., Nguyen, H.T., Cannon, S.B. and Stacey, G. (2007) Molecular evolution of lysin motif-type receptor-like kinases in plants. Plant Physiol., 144, 623-636.

Zhao, Z., Liu, H., Wang, C. and Xu, J.R. (2014) Correction to Comparative analysis of fungal genomes reveals different plant cell wall degrading capacity in fungi [BMC Genomics 14(2013) 274]. BMC Genomics, 15.

Zhong, R. and Ye, Z.H. (2015) Secondary cell walls: Biosynthesis, patterned deposition and transcriptional regulation. Plant Cell Physiol., 56, 195-214.

Zipfel, C., Kunze, G., Chinchilla, D., Caniard, A., Jones, J.D.G., Boller, T. and Felix, G. (2006) Perception of the Bacterial PAMP EF-Tu by the Receptor EFR Restricts AgrobacteriumMediated Transformation. Cell, 125, 749-760.

Zipfel, C., Robatzek, S., Navarro, L., Oakeley, E.J., Jones, J.D.G., Felix, G. and Boller, T. (2004) Bacterial disease resistance in Arabidopsis through flagellin perception. Nature, 428, 764-767. 


\section{Supplemental Material}

Table S1. Predicted motifs of GHs in Bgh.

\begin{tabular}{|c|c|c|}
\hline CWDE family & Predicted Motif & \\
\hline BluGen Protein Number & dbCAN & Motif Scan \\
\hline \multicolumn{3}{|l|}{ GH3 } \\
\hline BGH00678 & $\mathrm{GH} 3$ & - \\
\hline \multicolumn{3}{|l|}{ GH5 } \\
\hline BGH06688 & GH5_12 & $\begin{array}{l}\text { Cellulase Motif } \\
\text { Glycoside Hydrolase family } 5 \text { signature }\end{array}$ \\
\hline BGH06810 & GH5_9 & Bipartite nuclear localization signal profile \\
\hline BGH00680 & GH5_9 & Glycoside Hydrolase family 5 signature \\
\hline BGH00086 & GH5_9 & - \\
\hline \multicolumn{3}{|l|}{ GH13 } \\
\hline BGH00197 & $\begin{array}{l}\text { CBM48 } \\
\text { GH13_8 }\end{array}$ & $\begin{array}{l}\text { Alpha amylase, catalytic domain } \\
\text { Alpha amylase, C-terminal all-beta domain }\end{array}$ \\
\hline BGH00315 & GH13_40 & Alpha amylase, catalytic domain \\
\hline BGH000684000002001 & GH13_25 & Amylo-alpha-1,6-glucosidase \\
\hline BGH000061000002001 & GH13_40 & Alpha amylase, catalytic domain \\
\hline \multicolumn{3}{|l|}{ GH16 } \\
\hline BGH05640 & GH16 & Glycoside hydrolases family 16 motif \\
\hline BGH00719 & $\mathrm{GH} 16$ & $\begin{array}{l}\text { Glycoside hydrolases family } 16 \text { motif } \\
\text { Histidine rich profile }\end{array}$ \\
\hline BGH00720 & $\mathrm{GH} 16$ & Glycoside hydrolases family 16 motif \\
\hline BGH00726 & $\mathrm{GH} 16$ & Glycoside hydrolases family 16 motif \\
\hline BGH00729 & $\mathrm{GH} 16$ & $\begin{array}{l}\text { Glycoside hydrolases family } 16 \text { motif } \\
\text { Prokaryotic membrane lipoprotein lipid } \\
\text { attachment site profile }\end{array}$ \\
\hline BGH00731 & $\mathrm{GH} 16$ & Glycoside hydrolases family 16 motif \\
\hline BGH00732 & $\mathrm{GH} 16$ & $\begin{array}{l}\text { Glycoside hydrolases family } 16 \text { motif } \\
\text { Beta-glucan synthesis-associated protein } \\
\text { (SKN1) }\end{array}$ \\
\hline BGH01441 & $\mathrm{GH} 16$ & Glycoside hydrolases family 16 motif \\
\hline BGH05042 & $\mathrm{GH} 16$ & Glycoside hydrolases family 16 motif \\
\hline \multicolumn{3}{|l|}{ GH17 } \\
\hline BGH06298 & $\mathrm{GH} 17$ & Glycoside hydrolases family 17 motif \\
\hline
\end{tabular}




\begin{tabular}{|c|c|c|}
\hline BGH06777 & $\mathrm{GH} 17$ & Glycoside hydrolases family 17 motif \\
\hline BGH00219 & $\mathrm{GH} 17$ & Glycoside hydrolases family 17 motif \\
\hline BGH00220 & - & Glycoside hydrolases family 17 motif \\
\hline BGH00734 & $\mathrm{GH} 17$ & Threonine rich region \\
\hline BGH00736 & $\mathrm{GH} 17$ & - \\
\hline BGH05070 & $\mathrm{GH} 17$ & - \\
\hline BGH05207 & - & $\begin{array}{l}\text { Cytosolic fatty acid binding proteins signature } \\
\text { Prokaryotic membrane lipoprotein lipid } \\
\text { attachment site profile }\end{array}$ \\
\hline \multicolumn{3}{|l|}{ GH18 } \\
\hline BGH05372 & $\mathrm{GH} 18$ & $\begin{array}{l}\text { Glycoside hydrolases family } 18 \text { motif } \\
\text { Chitinases family } 18 \text { active site }\end{array}$ \\
\hline BGH00059 & $\mathrm{GH} 18$ & $\begin{array}{l}\text { Glycoside hydrolases family } 18 \text { motif } \\
\text { Chitin recognition or binding domain signature }\end{array}$ \\
\hline BGH00122 & $\mathrm{GH} 18$ & $\begin{array}{l}\text { Glycoside hydrolases family } 18 \text { motif } \\
\text { Chitinases family } 18 \text { active site } \\
\text { Chitin-binding type- } 1 \text { domain profile }\end{array}$ \\
\hline BGH00634 & $\mathrm{GH} 18$ & $\begin{array}{l}\text { Glycoside hydrolases family } 18 \text { motif } \\
\text { Chitinases family } 18 \text { active site }\end{array}$ \\
\hline BGH00329 & $\mathrm{GH} 18$ & $\begin{array}{l}\text { Glycoside hydrolases family } 18 \text { motif } \\
\text { Chitinases family } 18 \text { active site }\end{array}$ \\
\hline BGH00588 & $\mathrm{GH} 18$ & $\begin{array}{l}\text { Glycoside hydrolases family } 18 \text { motif } \\
\text { Chitinases family } 18 \text { active site } \\
\text { Chitin recognition or binding domain signature } \\
\text { and Chitin recognition protein }\end{array}$ \\
\hline BGH00738 & $\mathrm{GH} 18$ & $\begin{array}{l}\text { Glycoside hydrolases family } 18 \text { motif } \\
\text { Chitinases family } 18 \text { active site }\end{array}$ \\
\hline BGH00739 & $\mathrm{GH} 18$ & $\begin{array}{l}\text { Glycoside hydrolases family } 18 \text { motif } \\
\text { Chitinases family } 18 \text { active site }\end{array}$ \\
\hline BGH02531 & - & Serine-rich region profile \\
\hline \multicolumn{3}{|l|}{ GH31 } \\
\hline BGH06910 & GH31 & $\begin{array}{l}\text { Glycosyl hydrolases family } 31 \text { active site } \\
\text { Glycosyl hydrolases family } 31\end{array}$ \\
\hline \multicolumn{3}{|l|}{ GH37 } \\
\hline BGH006215000001001 & GH37 & - \\
\hline \multicolumn{3}{|l|}{ GH38 } \\
\hline BGH00758 & GH38 & - \\
\hline \multicolumn{3}{|l|}{ GH47 } \\
\hline BGH00762 & $\mathrm{GH} 47$ & Glycoside hydrolases family 47 motif \\
\hline $\mathrm{BGH} 00763$ & $\mathrm{GH} 47$ & Glycoside hydrolases family 47 motif \\
\hline
\end{tabular}




\begin{tabular}{|c|c|c|}
\hline BGH00764 & $\mathrm{GH} 47$ & Glycoside hydrolases family 47 motif \\
\hline BGH00768 & $\mathrm{GH} 47$ & Glycoside hydrolases family 47 motif \\
\hline \multicolumn{3}{|l|}{ GH55 } \\
\hline BGH00772 & GH55 & - \\
\hline \multicolumn{3}{|l|}{ GH61 / AA49 } \\
\hline BGH04794 & $\begin{array}{l}\text { AA9 } \\
\text { AA11 }\end{array}$ & Glycosyl hydrolase family 61 motif \\
\hline BGH02286 & AA11 & Proline-rich region profile \\
\hline \multicolumn{3}{|l|}{ GH63 } \\
\hline BGH02562 & GH63 & \\
\hline BGH004450000001001 & GH63 & $\begin{array}{l}\text { Endoplasmic reticulum targeting sequence } \\
\text { Mannosyl oligosaccharide glucosidase }\end{array}$ \\
\hline \multicolumn{3}{|l|}{ GH72 } \\
\hline BGH00774 & $\begin{array}{l}\text { GH72 } \\
\text { CBM43 }\end{array}$ & $\begin{array}{l}\text { Glycolipid anchored surface protein (GAS1) } \\
\text { X8 domain }\end{array}$ \\
\hline BGH00775 & GH72 & $\begin{array}{l}\text { Glycolipid anchored surface protein (GAS1) } \\
\text { Cellulase (glycosyl hydrolase family 5) }\end{array}$ \\
\hline BGH00776 & GH72 & Glycolipid anchored surface protein (GAS1) \\
\hline \multicolumn{3}{|l|}{ GH76 } \\
\hline BGH00778 & GH76 & Glycosyl hydrolase family 76 motif \\
\hline BGH00780 & GH76 & - \\
\hline BGH00782 & GH76 & Glycosyl hydrolase family 76 motif \\
\hline BGH00783 & GH76 & Glycosyl hydrolase family 76 motif \\
\hline BGH05252 & GH76 & Glycosyl hydrolase family 76 motif \\
\hline $\mathrm{BGH} 0006450$ & GH76 & Glycosyl hydrolase family 76 motif \\
\hline BGH00779 & GH76 & $\begin{array}{l}\text { Glycosyl hydrolase family } 76 \text { motif } \\
\text { Trp-Asp (WD) repeats signature }\end{array}$ \\
\hline \multicolumn{3}{|l|}{ GH78 } \\
\hline BGH000684000001001 & $\mathrm{GH} 78$ & - \\
\hline \multicolumn{3}{|l|}{ GH81 } \\
\hline BGH004744 & GH81 & - \\
\hline \multicolumn{3}{|l|}{ GH92 } \\
\hline BGH00795 & GH92 & Glycosyl hydrolase family 92 motif \\
\hline
\end{tabular}

${ }^{4} \mathrm{~A} A$ - Auxiliary Activity 


\begin{tabular}{lll}
\hline BGH006074000001001 & GH92 & $\begin{array}{l}\text { Glycosyl hydrolase family 92 motif } \\
\text { EF-hand calcium-binding domain }\end{array}$ \\
\hline GH93 & & \\
\hline BGH006353 & GH93 \\
GH74 & - \\
\hline
\end{tabular}


Table S2. In planta RPM values of $\mathrm{Bgh}$ GH families 5, 16, 17, 47 and 76 upon 6, 12, 18 and 24 hpi. The Arabidopsis triple mutant pen2 pad4 sag101 expressing MLA1 was infected with the Bgh isolate A6. Samples were taken upon 6, 12, 18 and 24 hpi, RNA was extracted and RNA sequencing was performed (Hacquard et al., 2013). RPM values were calculated from the raw data available at the National Center for Biotechnology Information Gene Expression Omnibus (GEO) database.

\begin{tabular}{|c|c|c|c|c|}
\hline \multirow{2}{*}{$\begin{array}{c}\text { GH family } \\
\text { BluGen Gen } \\
\text { Number }\end{array}$} & \multicolumn{4}{|c|}{ RPM Values (compatible interaction) } \\
\hline & $6 \mathrm{hpi}$ & $12 \mathrm{hpi}$ & $18 \mathrm{hpi}$ & $24 \mathrm{hpi}$ \\
\hline \multicolumn{5}{|l|}{ GH5 } \\
\hline Bgh06688 & 301 & 69 & 140 & 78 \\
\hline Bgh06810 & 3 & 8 & 15 & 21 \\
\hline Bgh00680 & 6739 & 5333 & 6786 & 6696 \\
\hline Bgh00086 & 375 & 329 & 1272 & 1098 \\
\hline \multicolumn{5}{|l|}{ GH16 } \\
\hline Bgh05640 & 67 & 459 & 919 & 611 \\
\hline Bgh00719 & 918 & 251 & 477 & 639 \\
\hline Bgh00720 & 6867 & 15403 & 6578 & 4777 \\
\hline Bgh00726 & 735 & 196 & 307 & 368 \\
\hline Bgh00729 & 1643 & 443 & 1147 & 1235 \\
\hline Bgh00731 & 1308 & 1453 & 1319 & 1111 \\
\hline Bgh00732 & 67 & 79 & 102 & 169 \\
\hline Bgh01441 & 3357 & 797 & 796 & 495 \\
\hline Bgh05042 & 31 & 125 & 361 & 2140 \\
\hline \multicolumn{5}{|l|}{ GH17 } \\
\hline Bgh06298 & 351 & 452 & 740 & 454 \\
\hline Bgh06777 & 16 & 1301 & 1053 & 606 \\
\hline Bgh00219 & 3 & 63 & 115 & 157 \\
\hline Bgh00220 & 98 & 1552 & 369 & 145 \\
\hline Bgh00734 & 906 & 470 & 727 & 595 \\
\hline Bgh00736 & 43 & 4409 & 5580 & 4357 \\
\hline Bgh05070 & 642 & 3873 & 1464 & 823 \\
\hline Bgh05207 & 49 & 3720 & 1770 & 1387 \\
\hline \multicolumn{5}{|l|}{ GH47 } \\
\hline Bgh00762 & 8983 & 3695 & 3885 & 4707 \\
\hline Bgh00763 & 2338 & 861 & 1116 & 870 \\
\hline Bgh00764 & 1324 & 1691 & 1033 & 939 \\
\hline Bgh00768 & 5992 & 4560 & 5743 & 7606 \\
\hline \multicolumn{5}{|l|}{ GH76 } \\
\hline Bgh00778 & 1619 & 464 & 757 & 868 \\
\hline Bgh00780 & 1060 & 1270 & 1881 & 1634 \\
\hline Bgh00782 & 450 & 1185 & 448 & 137 \\
\hline Bgh00783 & 50 & 37 & 46 & 27 \\
\hline Bgh05252 & 0 & 0 & 0 & 0 \\
\hline Bgh0006450 & 5650 & 204 & 316 & 340 \\
\hline
\end{tabular}

${ }^{5}$ Gene identification numbers were retrieved from www.blugen.org. 
A

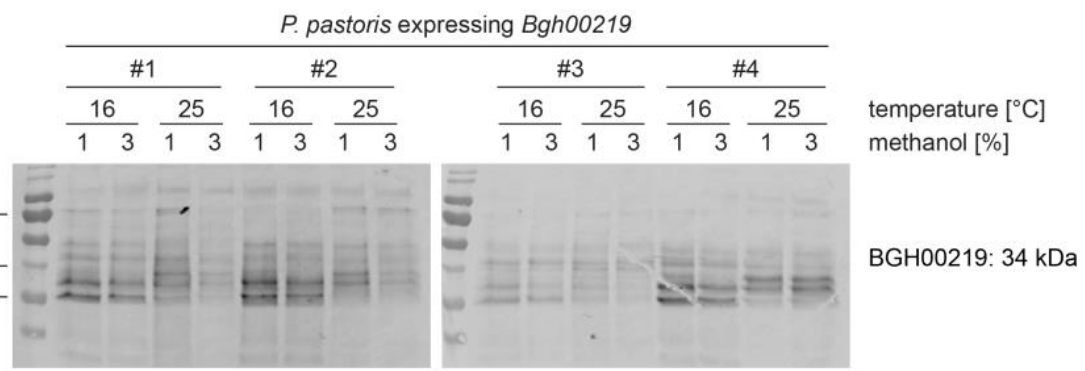

B

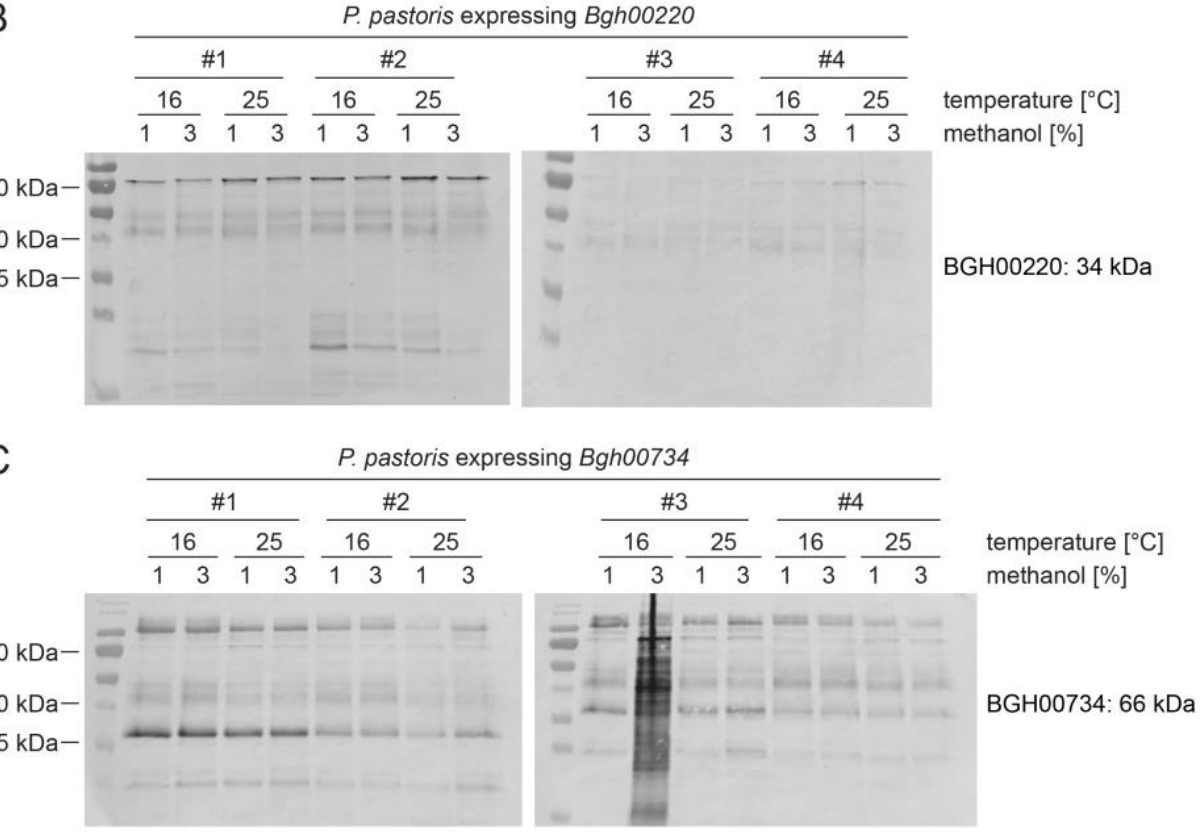

D

P. pastoris expressing Bgh00736

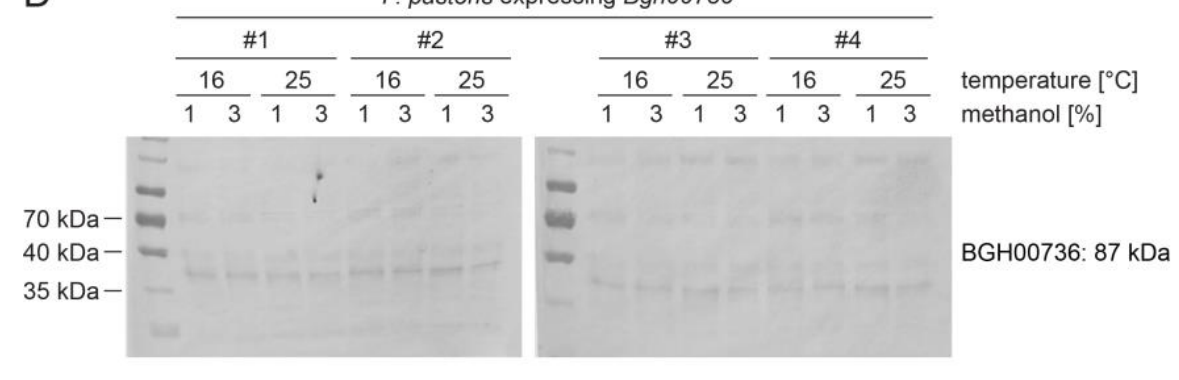

$\mathrm{E}$

P. pastoris expressing Bgh06777

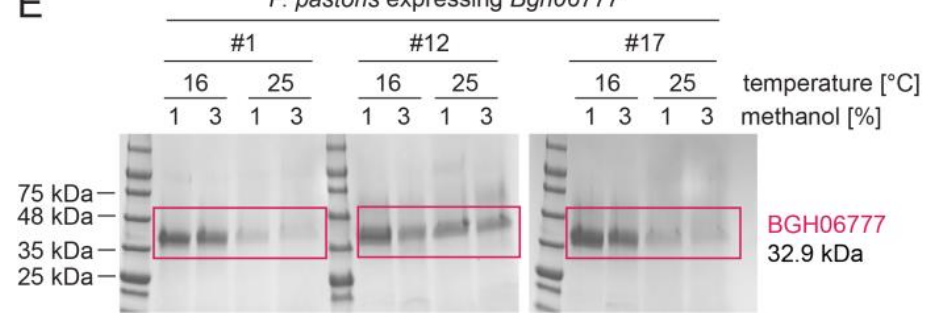

Figure S1. Small scale expression of Bgh GH17 genes. Three to four clones were tested for the expression of either Bgh00219 (A), Bgh00220 (B), Bgh00734 (C), Bgh00736 (D) or Bgh06777 (E). All clones were grown in YPD at $30^{\circ} \mathrm{C}$ overnight and then re-inoculated in BMGY and further incubated overnight. The pellet was resuspended in $\mathrm{BMMY}$ containing either $1 \%$ or $3 \%$ methanol to induce the expression and the culture was incubated at either $16^{\circ} \mathrm{C}$ or $25^{\circ} \mathrm{C}$ for three to five days. $1 \%$ or $3 \%$ methanol was added to the cultures every $24 \mathrm{~h}$. Upon 3 to 5 days, the supernatant of the cultures was analysed via SDS PAGE and Western Blot. To visualize the presence of the proteins, the membranes or the gel were directly stained with Coomassie Brilliant Blue. 


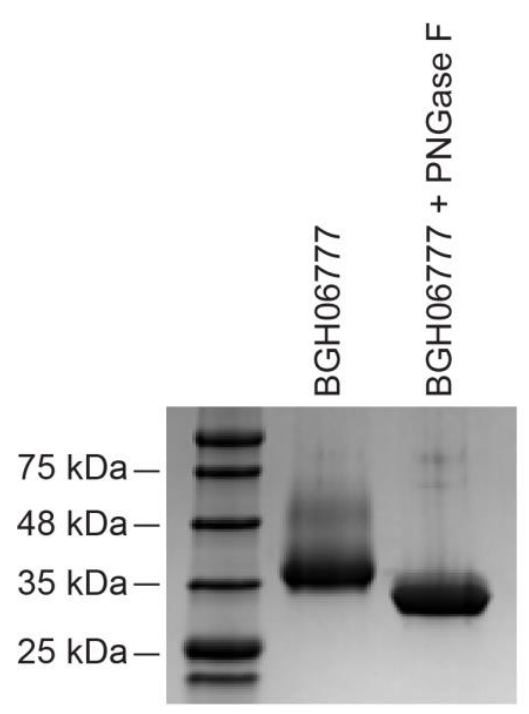

Figure S2. SDS PAGE analysis of recombinant BGH06777. $9 \mu \mathrm{g}$ recombinant protein before deglycosylation with PNGase F and $9 \mu \mathrm{g}$ BGH06777 after deglycosylation with PNGase F. To visualize the protein, the SDS gel was stained with Coomassie Brilliant Blue. 

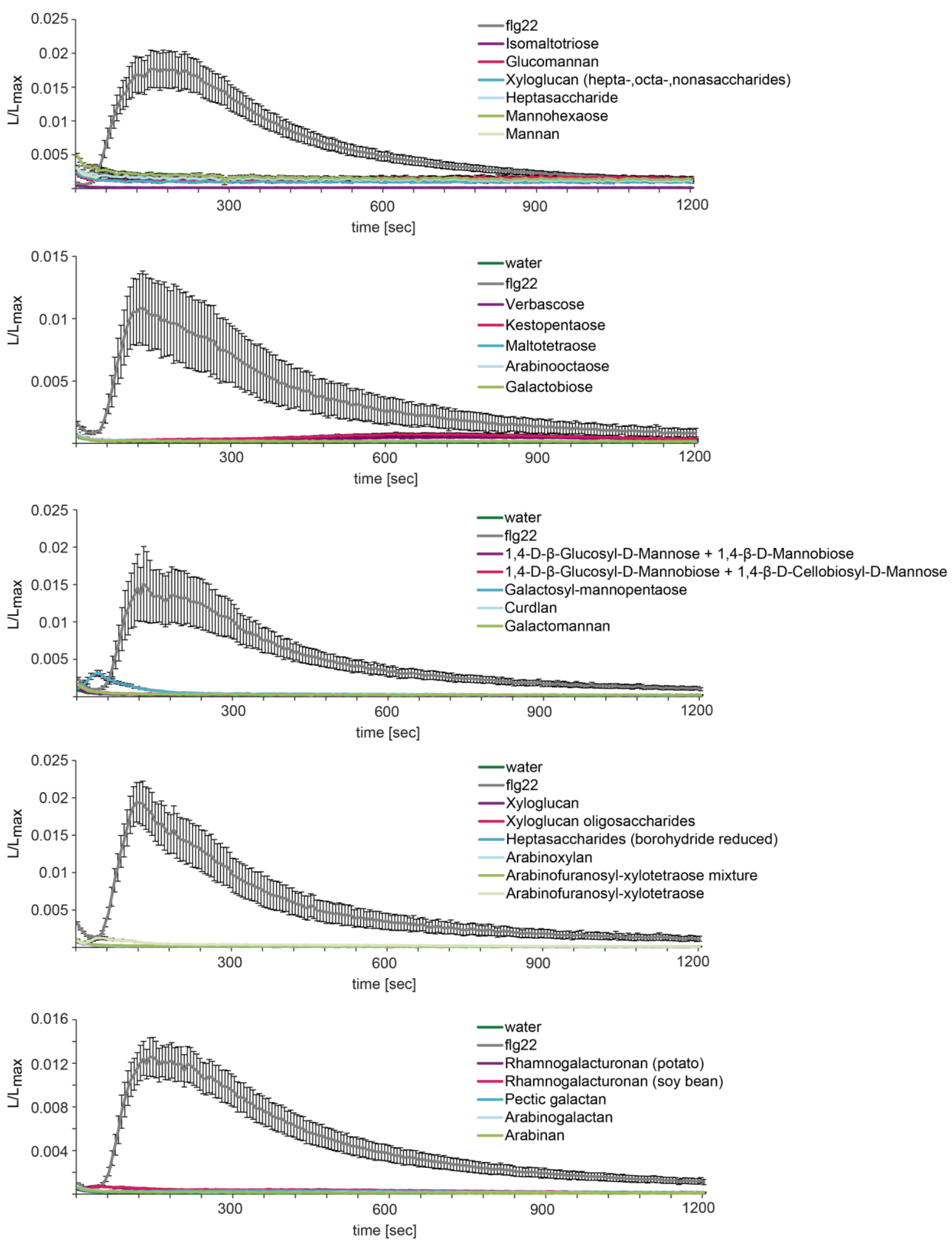

Figure S3. Influx of $\mathrm{Ca}^{2+}$ in Col-0 upon oligo- and polysaccharide treatment. 8-10-day old Arabidopsis Col-0 seedlings expressing the calcium sensor protein aequorin were treated with water, $50 \mathrm{nM}$ flg22 and $100 \mu \mathrm{g} \mathrm{ml}^{-1}$ of the respective oligosaccharide or polysaccharide as depicted. The $\mathrm{Ca}^{2+}$ elevation was measured every $6 \mathrm{sec}$ for $1200 \mathrm{sec}(\mathrm{L})$. To obtain the total remaining luminescence $\left(L_{\max }\right)$, the remaining aequorin was discharged by adding $\mathrm{CaCl}_{2}$ to each well and luminescence was recorded for $3 \mathrm{~min}$ in $6 \mathrm{sec}$ intervals. For normalization, the elicitor induced luminescence per $6 \mathrm{sec}(\mathrm{L})$ was divided by $L_{\max }$. The data shown represents the mean of 12 seedlings and error bars represent SEM. The experiments were performed once. 

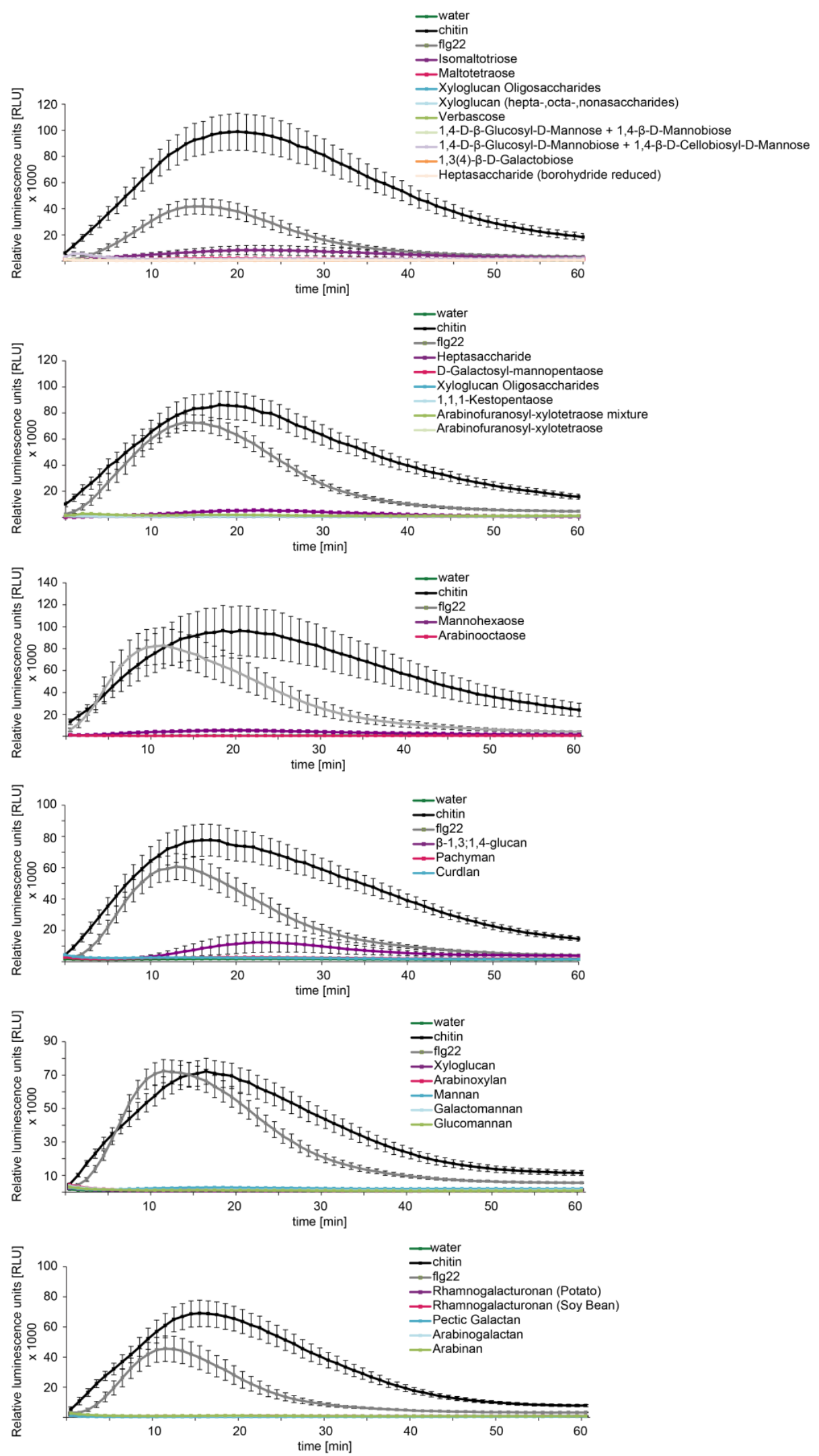

Figure S4. Generation of ROS in Col-0 upon oligo- and polysaccharide treatment. Leaf discs of 5-7 week old Arabidopsis Col-0 plants were treated with water, $100 \mathrm{\mu g} \mathrm{ml}^{-1}$ chitin, $100 \mathrm{nM}$ flg22 or $100 \mathrm{\mu g} \mathrm{ml}^{-1}$ of the respective oligo- or polysaccharide as indicated. Relative luminescence units (RLU) were recorded every minute for $60 \mathrm{~min}$. The shown data represent the mean of eight leaf discs with SEM. The experiment were performed once. 

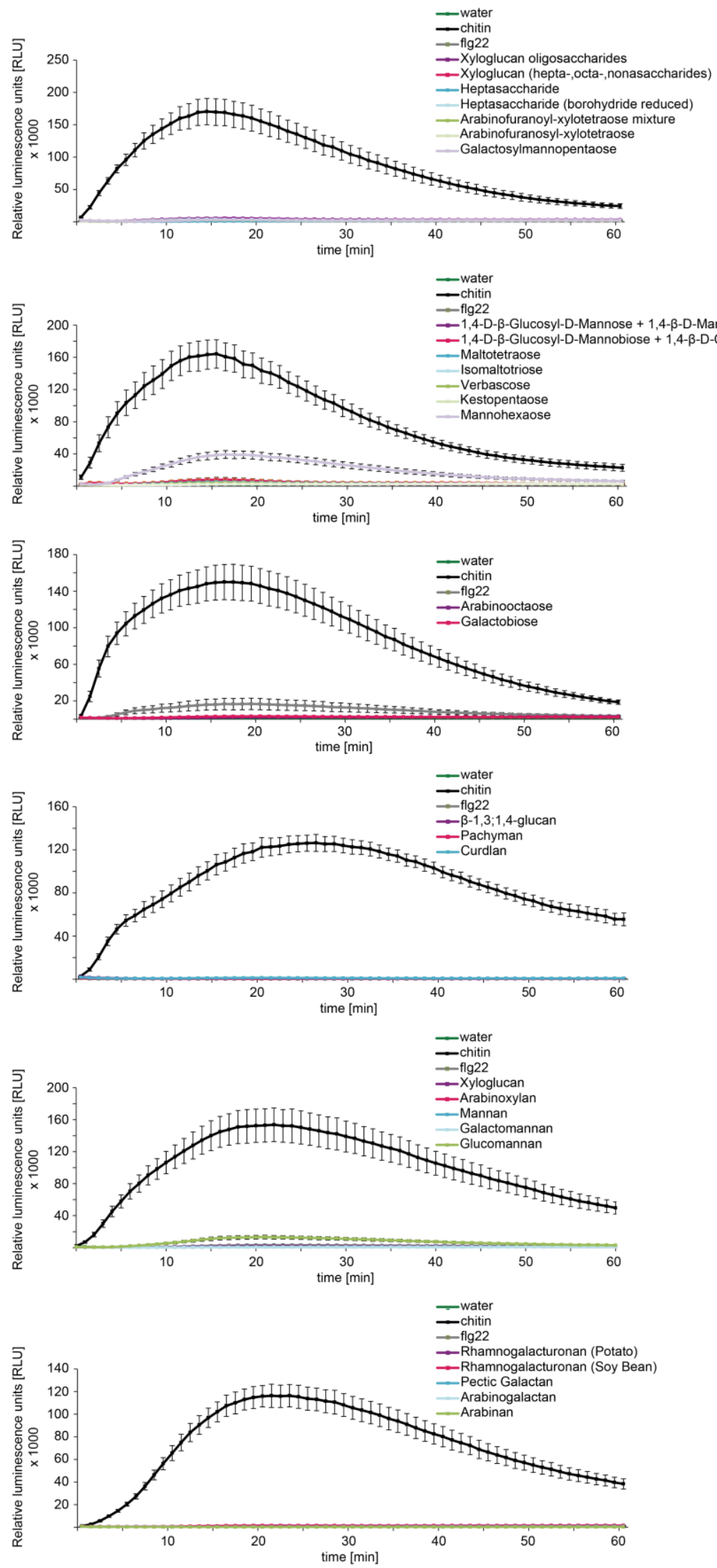

Figure S5. Generation of ROS in Ws-0 upon oligo- and polysaccharide treatment. Leaf discs of 5-7 week old Arabidopsis Ws-0 plants were treated with water, $100 \mathrm{\mu g} \mathrm{ml}^{-1}$ chitin, $100 \mathrm{nM}$ flg22 or $100 \mu \mathrm{g} \mathrm{ml}^{-1}$ of the respective oligo- or polysaccharide as indicated. Relative luminescence units (RLU) recorded every minute for $60 \mathrm{~min}$. The shown data represent the mean of eight leaf discs with SEM. The experiment was performed once. 

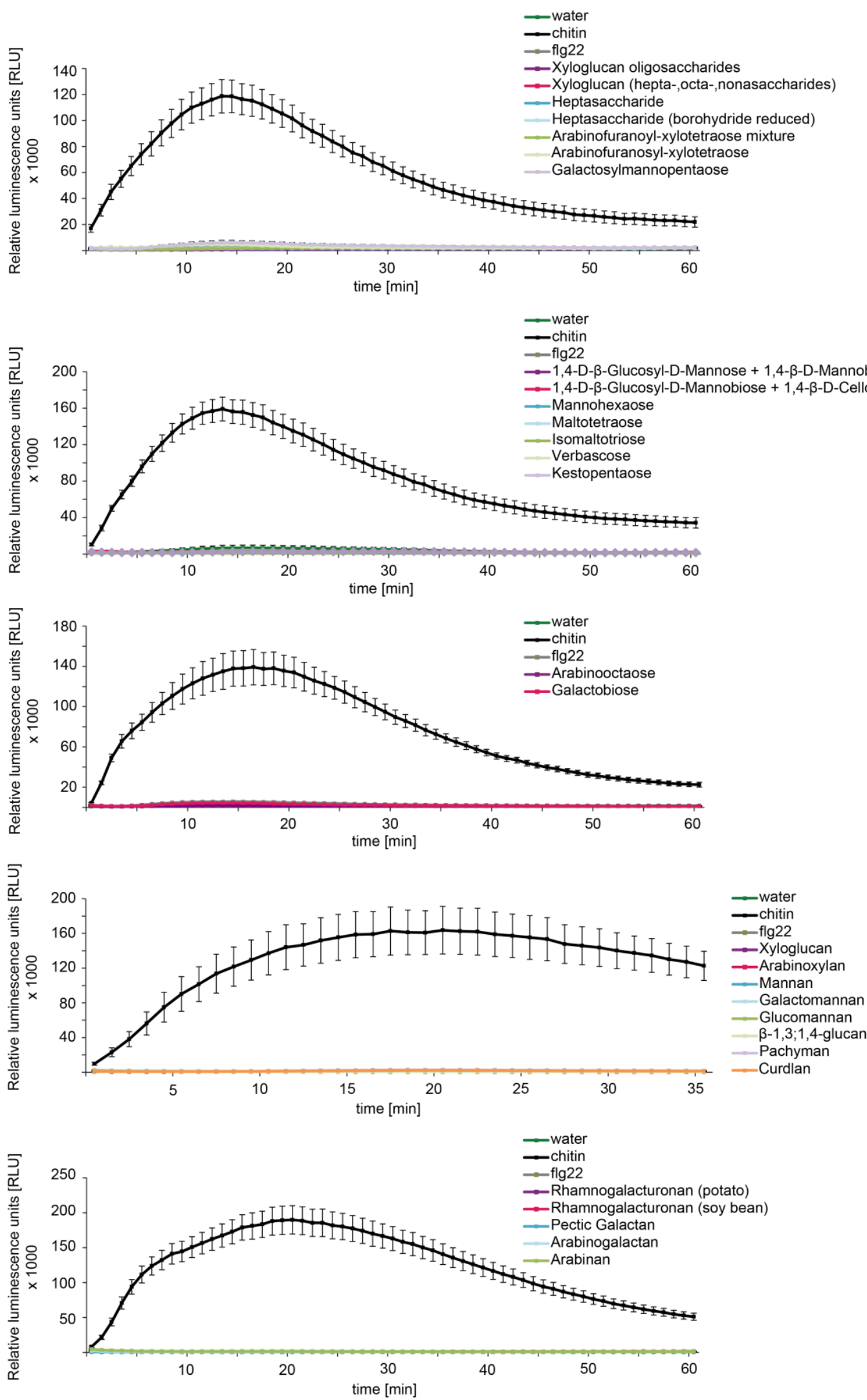

Figure S6. Generation of ROS in Ws-4 upon oligo- and polysaccharide treatment. Leaf discs of 5-7 week old Arabidopsis Ws-4 plants were treated with water, $100 \mathrm{\mu g} \mathrm{ml}^{-1}$ chitin, $100 \mathrm{nM} \mathrm{flg22} \mathrm{or} 100 \mu \mathrm{g} \mathrm{ml}^{-1}$ of the respective oligo- or polysaccharide as indicated. Relative luminescence units (RLU) were recorded every minute for 60 or $35 \mathrm{~min}$. The shown data represent the mean of eight leaf discs with SEM. The experiment was performed once. 


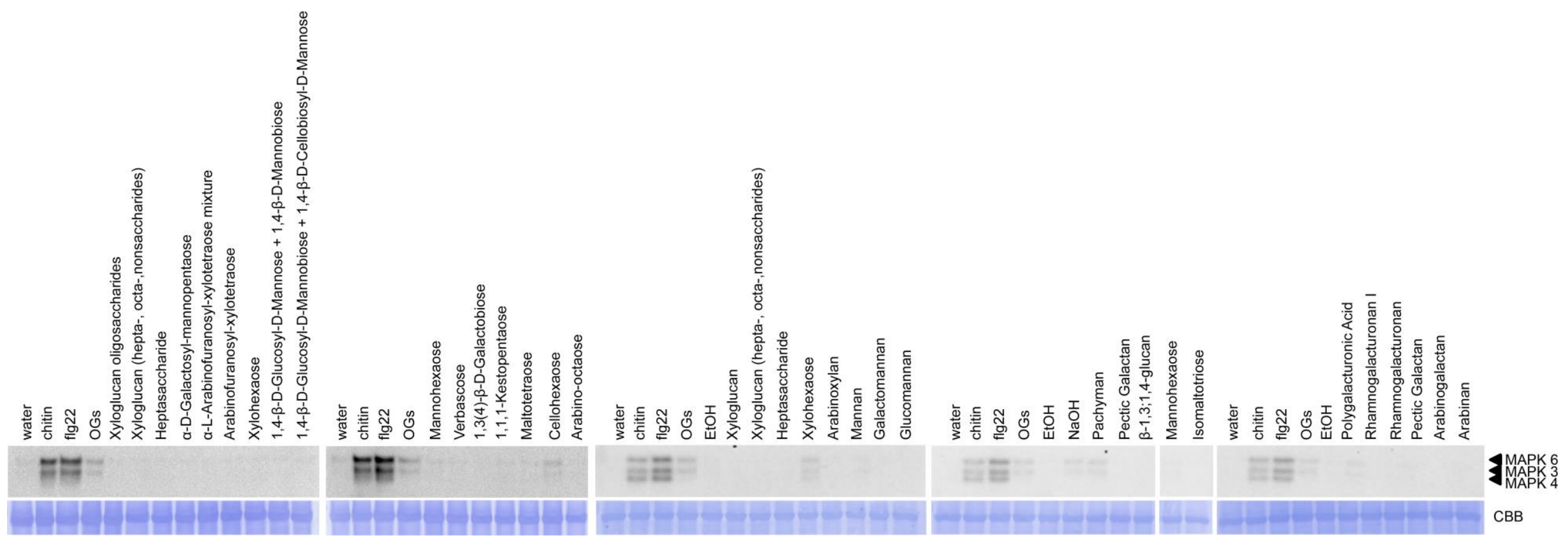

Figure S7. MAPK activation in Col-0 upon treatment with several oligo- and polysaccharides. 14-day old in-vitro grown Arabidopsis Col-0 seedlings were treated with water, $10 \mu \mathrm{g} \mathrm{ml}^{-1}$ chitin, $50 \mathrm{nM}$ flg22 or $10 \mu \mathrm{g} \mathrm{ml}-1$ of the respective carbohydrate for 12 min. Activation of MAPK was analysed via immunoblot analyses using the p44/42-antibody. Lower panel shows Coomassie Brilliant Blue (CBB) staining as loading control. The experiment was performed once or twice. 


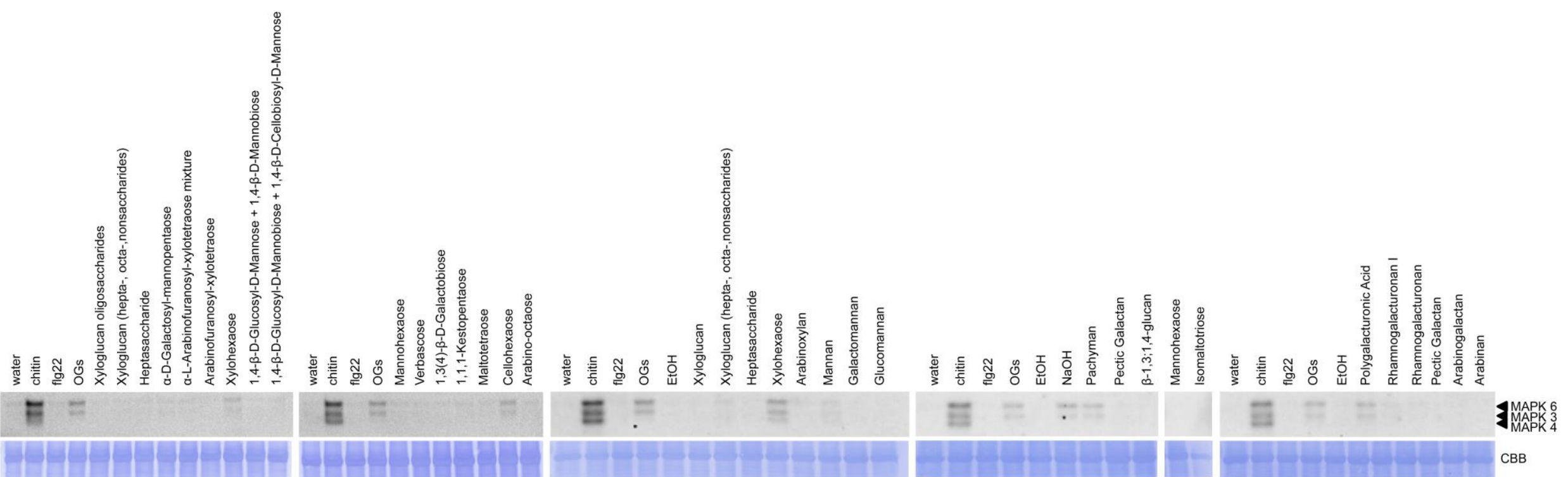

Figure S8. MAPK activation in Ws-0 upon treatment with several oligo- and polysaccharides. 14-day old in-vitro grown Arabidopsis Ws-0 seedlings were treated with water, $10 \mu \mathrm{g} \mathrm{ml}^{-1}$ chitin, $50 \mathrm{nM}$ flg22 or $10 \mu \mathrm{g} \mathrm{ml}$ - $^{-1}$ of the respective carbohydrate for 12 min. Activation of MAPK was analysed via immunoblot analyses using the p44/42-antibody. Lower panel shows Coomassie Brilliant Blue (CBB) staining as loading control. The experiment was performed once. 


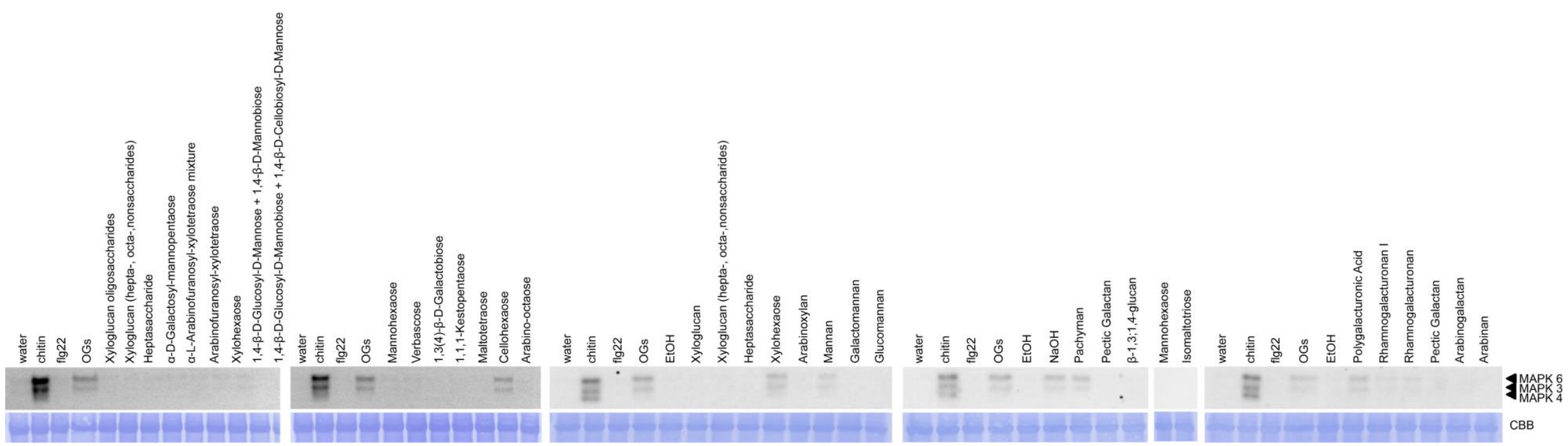

Figure S9. MAPK activation in Ws-4 upon treatment with several oligo- and polysaccharides. 14-day old in-vitro grown Arabidopsis Ws-4 seedlings were treated with water, $10 \mu \mathrm{g} \mathrm{ml}^{-1}$ chitin, $50 \mathrm{nM}$ flg22 or $10 \mu \mathrm{g} \mathrm{ml}{ }^{-1}$ of the respective carbohydrate for $12 \mathrm{~min}$. Activation of MAPK was analysed via immunoblot analyses using the p44/42-antibody. Lower panel shows Coomassie Brilliant Blue (CBB) staining as loading control. The experiment was performed once. 
A

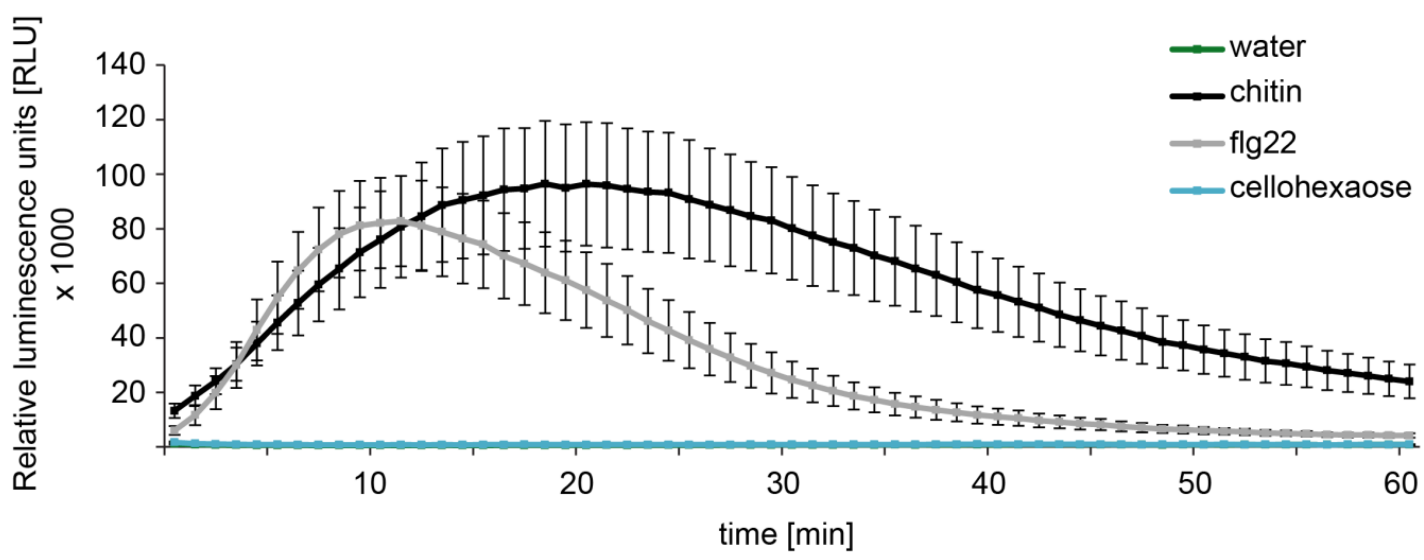

B

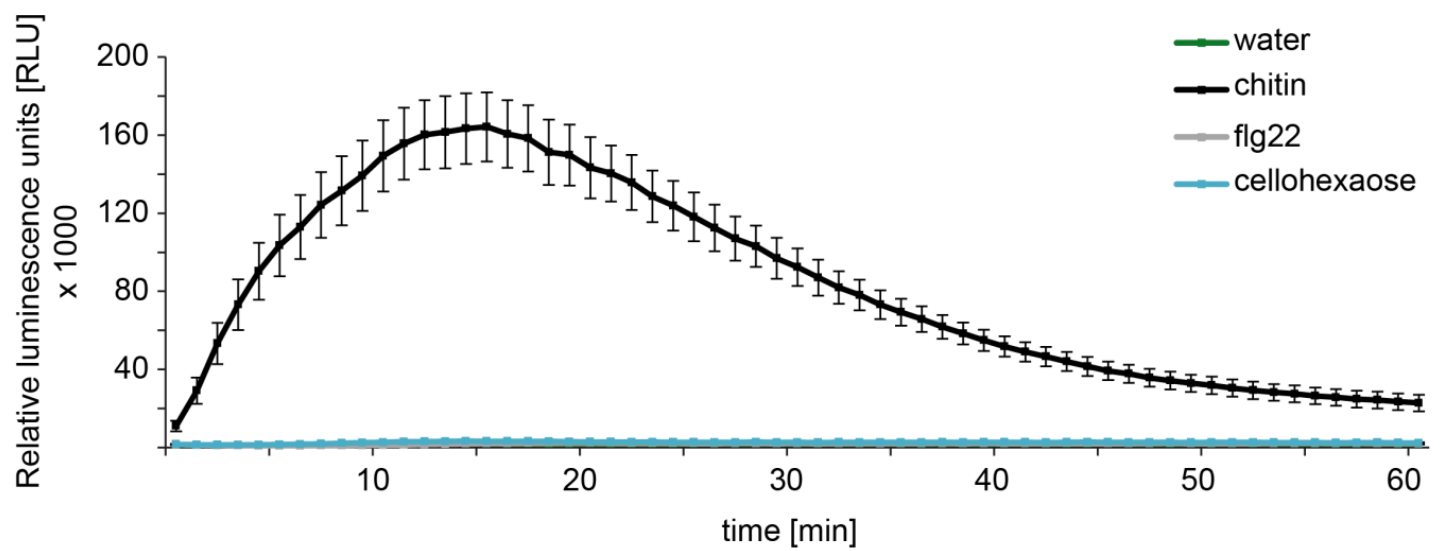

C

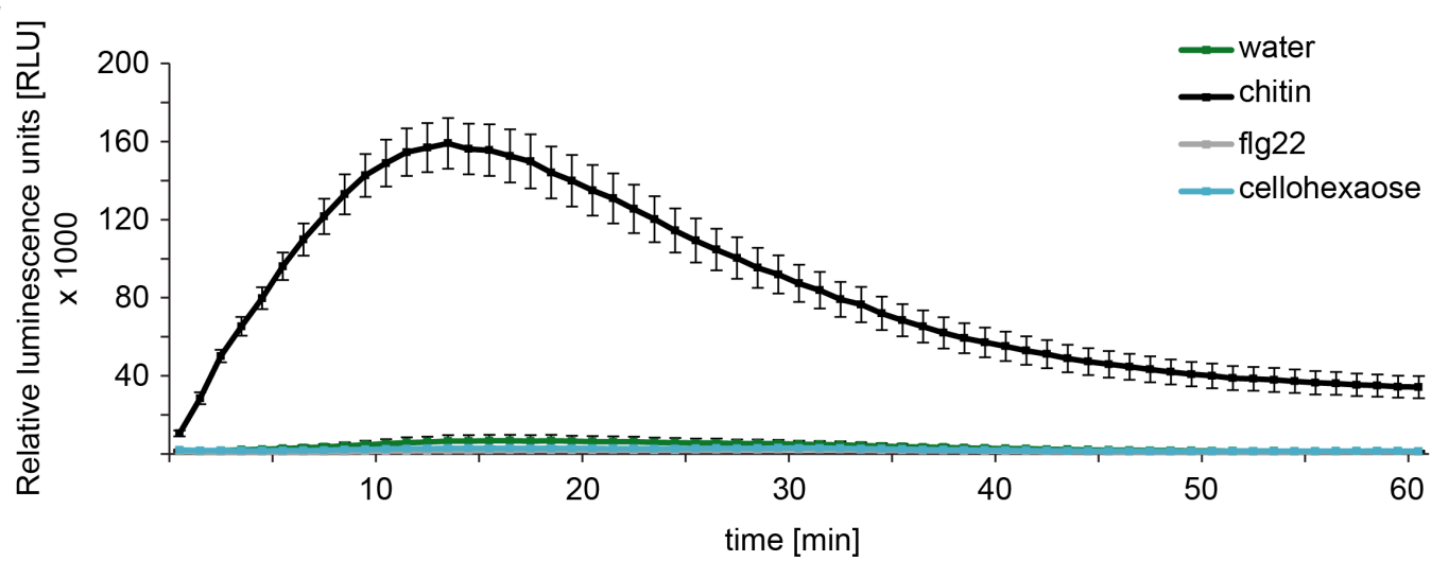

Figure S10. ROS burst generation in three Arabidopsis ecotypes upon cellohexaose treatment. Leaf discs of 5-7 week old Arabidopsis Col-0 (A), Ws-0 (B) or Ws-4 (C) plants were treated with water, $100 \mu \mathrm{g} \mathrm{ml}^{-1} \mathrm{chitin}$ $100 \mathrm{nM}$ flg22 or $100 \mathrm{~g} \mathrm{ml}^{-1}$ cellohexaose. Relative luminescence units (RLU) were recorded every minute for $60 \mathrm{~min}$. The shown data represent the mean of eight leaf discs with SEM. The experiment was performed once. 


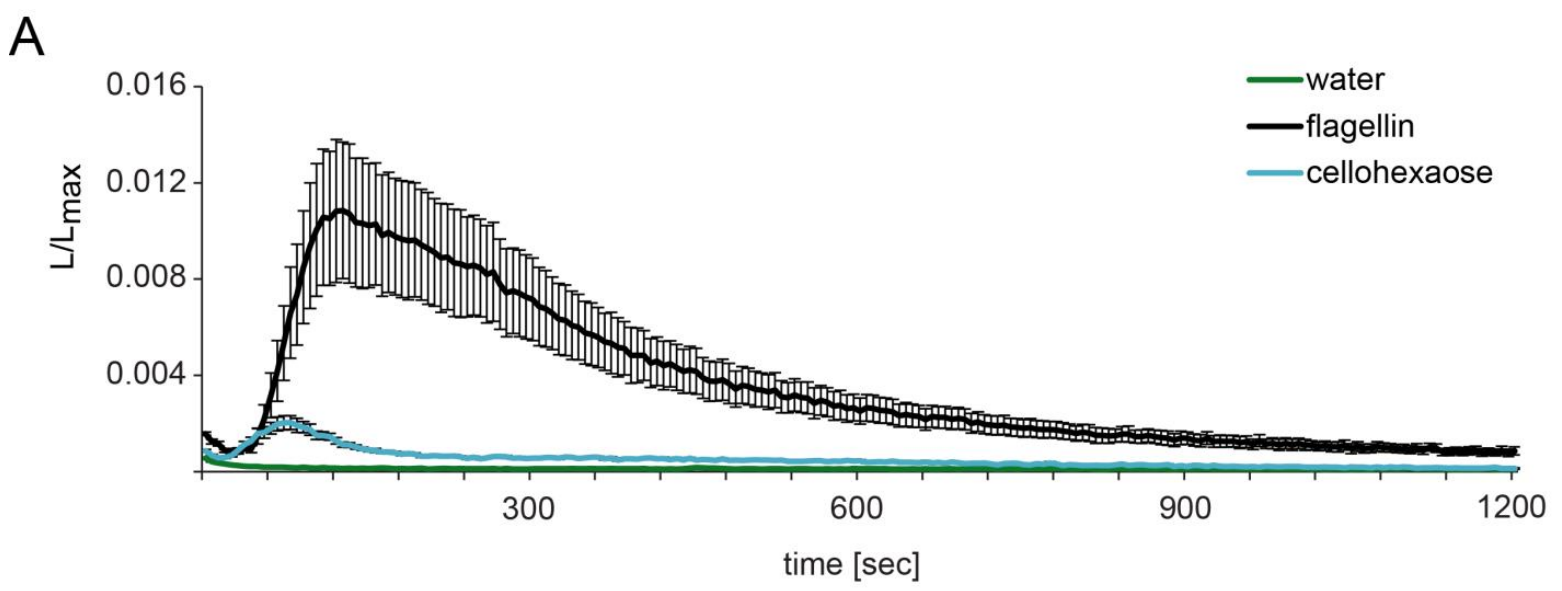

B

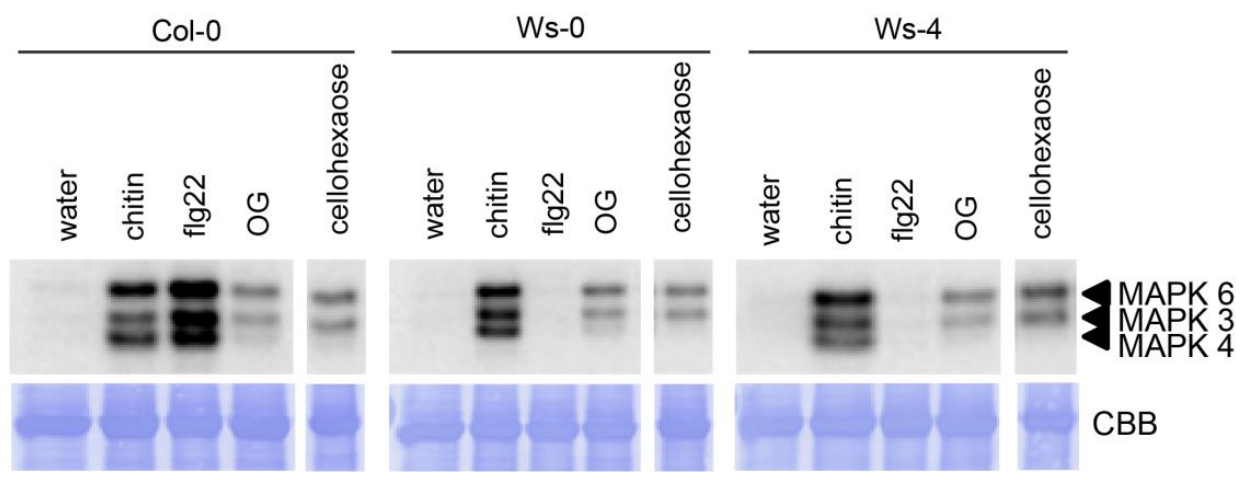

Figure S11. Activation of immune responses upon cellohexaose elicitation. (A) Elevation of intracellular $\mathrm{Ca}^{2+}$. 8-10 day old Arabidopsis seedlings expressing the $\mathrm{Ca}^{2+}$ sensor protein aequorin were treated with water, $100 \mathrm{nM}$ flg22 or $100 \mathrm{mg} \mathrm{ml}^{-1}$ cellohexaose. Elevation of $\mathrm{Ca}^{2+}$ was measured for $1200 \mathrm{sec}$ every $6 \mathrm{sec}$ (L). To obtain the total remaining luminescence $\left(\mathrm{L}_{\max }\right)$, the remaining aequorin was discharged by adding $\mathrm{CaCl}_{2}$ to each well and luminescence was recorded for $3 \mathrm{~min}$ in $6 \mathrm{sec}$ intervals. For normalization, the elicitor induced luminescence per $6 \mathrm{sec}(\mathrm{L})$ was divided by $L_{\max }$. The data shown represents the mean of 12 seedlings and error bars represent SEM. The experiment was performed once. (B) MAPK activation upon cellohexaose treatment. 14-day old in-vitro grown Arabidopsis Ws-0 and Ws-4 seedlings were treated with water, $10 \mu \mathrm{gl}^{-1} \mathrm{chitin}$ $50 \mathrm{nM}$ flg22 or $10 \mathrm{\mu g} \mathrm{ml}^{-1}$ cellohexaose for $12 \mathrm{~min}$. Activation of MAPK was analysed via immunoblot analyses using the p44/42-antibody. Lower panel shows Coomassie Brilliant Blue (CBB) staining as loading control. The experiment was performed twice with similar results. 
A

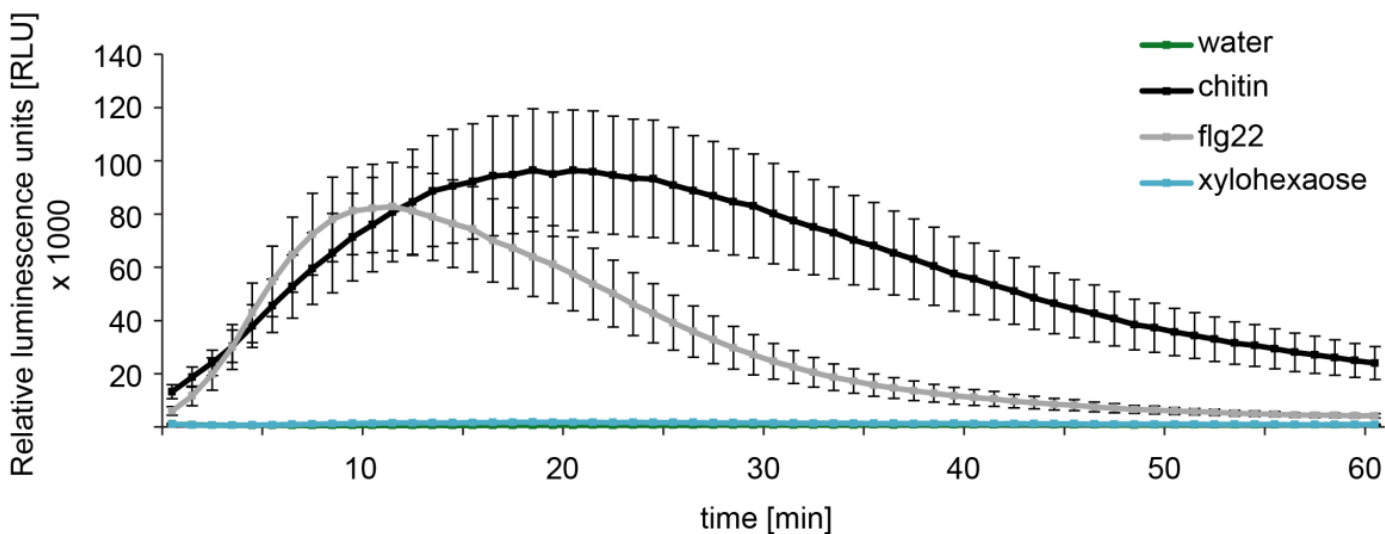

B

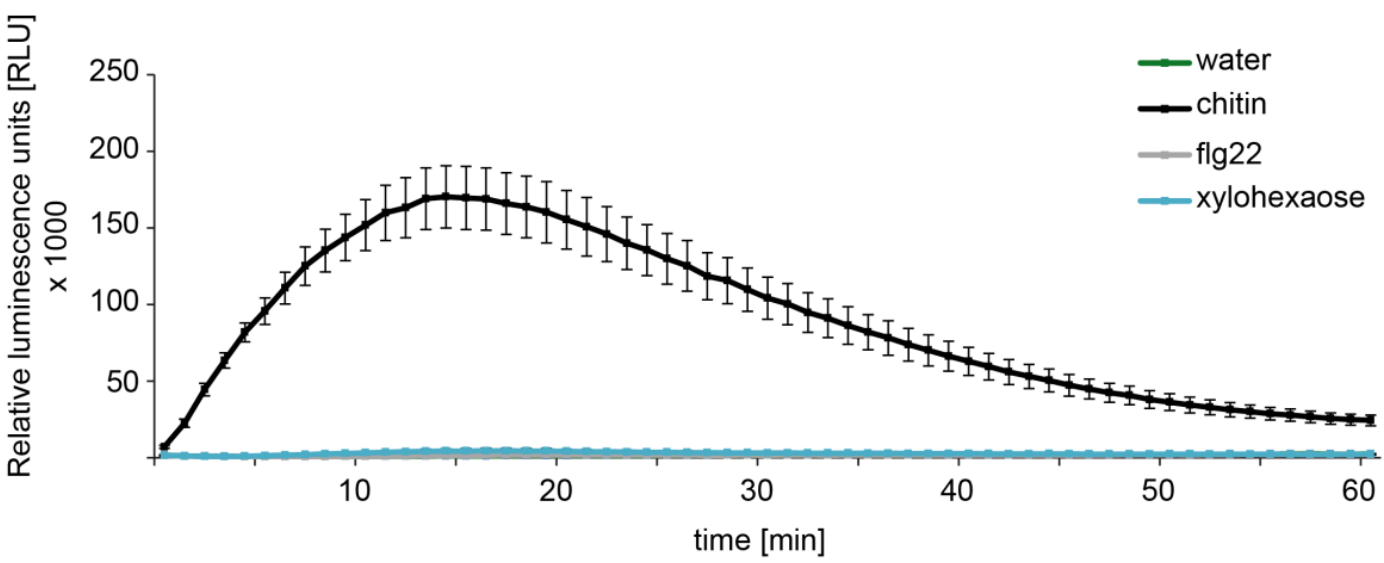

C

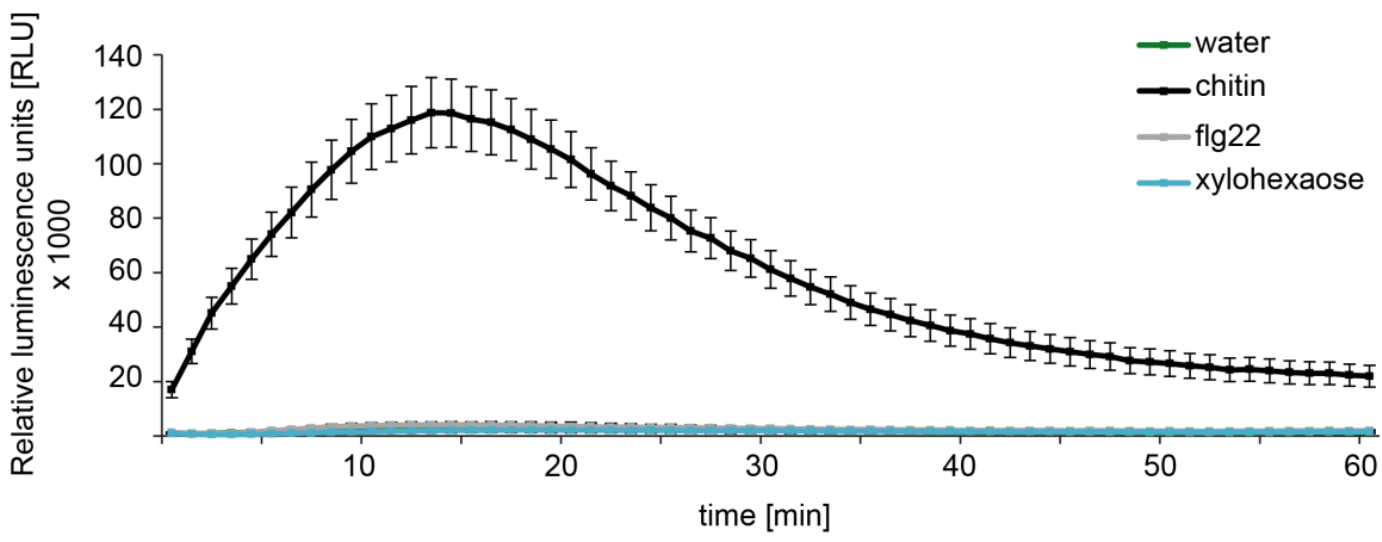

Figure S12. ROS burst generation upon xylohexaose treatment in different Arabidopsis ecotypes. Leaf discs of 5-7 week old Arabidopsis Col-0 (A), Ws-0 (B) or Ws-4 (C) plants were treated with water, $100 \mathrm{\mu g} \mathrm{ml}^{-1}$ chitin, $100 \mathrm{nM}$ flg22 or $100 \mu \mathrm{g} \mathrm{ml}^{-1}$ xylohexaose. Relative light units were recorded every minute for $60 \mathrm{~min}$. The shown data represent the mean of eight leaf discs with SEM. The experiment was performed once. 


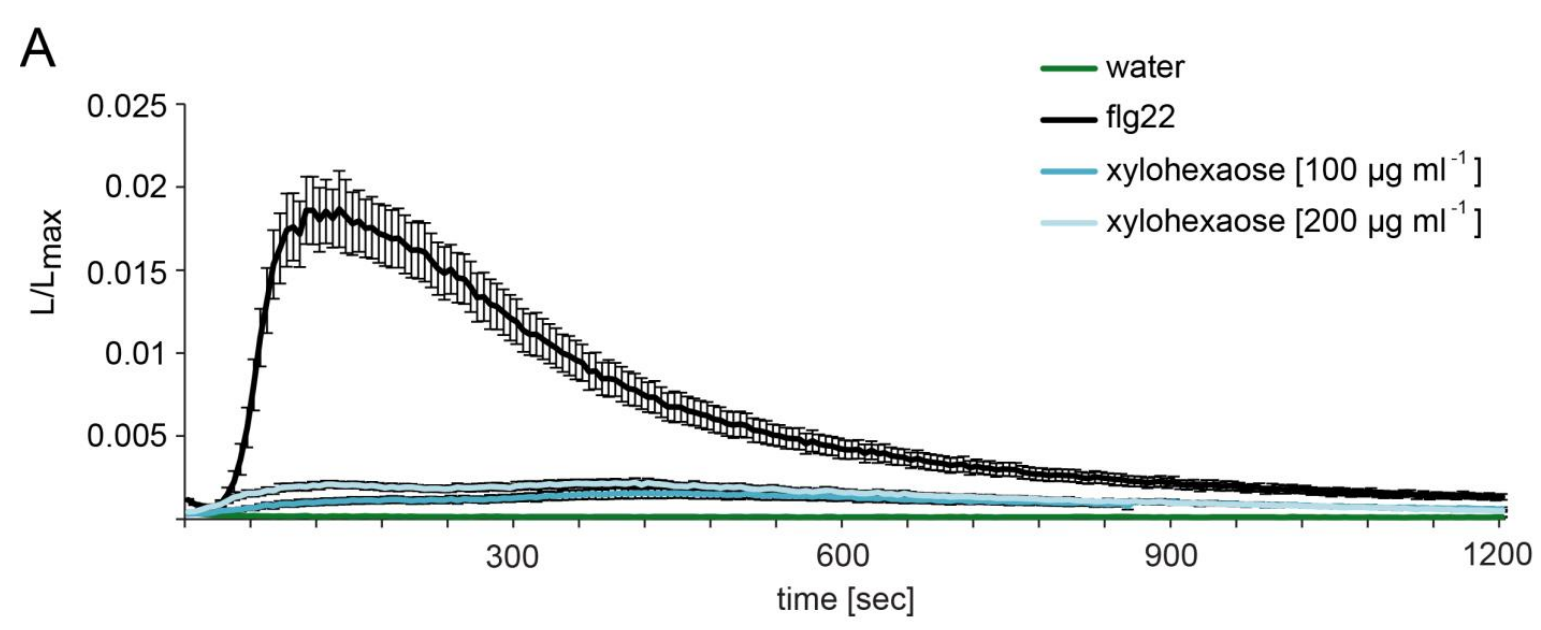

B
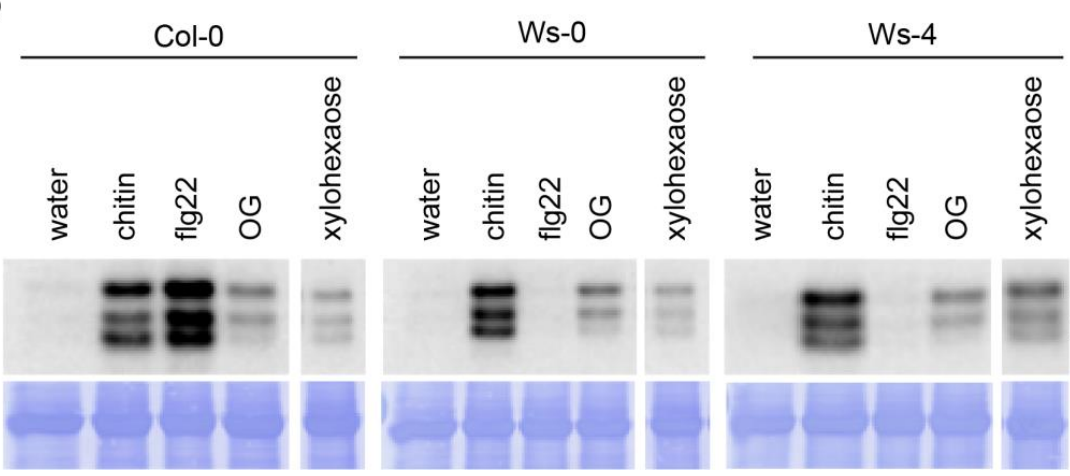

Figure S13. $\mathrm{Ca}^{2+}$ influx and MAPK activation upon xylohexaose elicitation. (A) Elevation of intracellular $\mathrm{Ca}^{2+}$. 8-10 day old Arabidopsis seedlings expressing the $\mathrm{Ca}^{2+}$ sensor protein aequorin were treated with water, $100 \mathrm{nM}$ flg22, $100 \mathrm{mg} \mathrm{ml}^{-1}$ xylohexaose or $200 \mu \mathrm{g} \mathrm{ml}^{-1}$ xylohexaose. Elevation of $\mathrm{Ca}^{2+}$ was measured for $1200 \mathrm{sec}$ every $6 \mathrm{sec}(\mathrm{L})$. To obtain the total remaining luminescence $\left(\mathrm{L}_{\max }\right)$, the remaining aequorin was discharged by adding $\mathrm{CaCl}_{2}$ to each well and luminescence was recorded for $3 \mathrm{~min}$ in $6 \mathrm{sec}$ intervals. For normalization, the elicitor induced luminescence per $6 \mathrm{sec}(\mathrm{L})$ was divided by $L_{\max }$. The data shown represents the mean of 12 seedlings and error bars represent SEM. The experiment was performed twice with similar results. (B) MAPK activation upon xylohexaose treatment. 14-day old in-vitro grown Arabidopsis Ws-0 and Ws-4 seedlings were treated with water, $10 \mu \mathrm{g} \mathrm{ml}^{-1}$ chitin, $50 \mathrm{nM}$ flg22 or $10 \mu \mathrm{g} \mathrm{ml}^{-1}$ xylohexaose for $12 \mathrm{~min}$. Activation of MAPK was analysed via immunoblot analyses using the p44/42-antibody. Lower panel shows Coomassie Brilliant Blue (CBB) staining as loading control. The experiment was performed twice with similar results. 
A

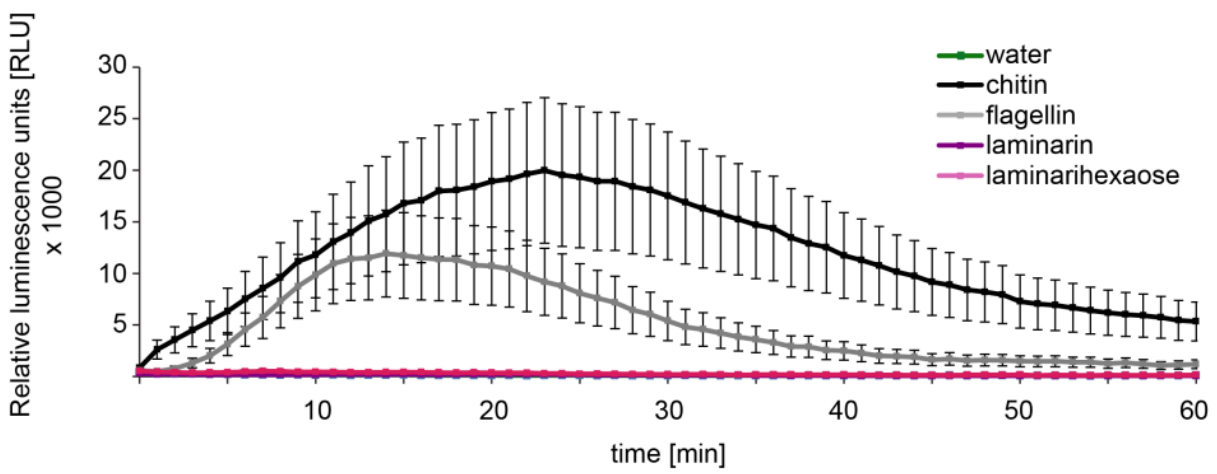

$\mathrm{B}$

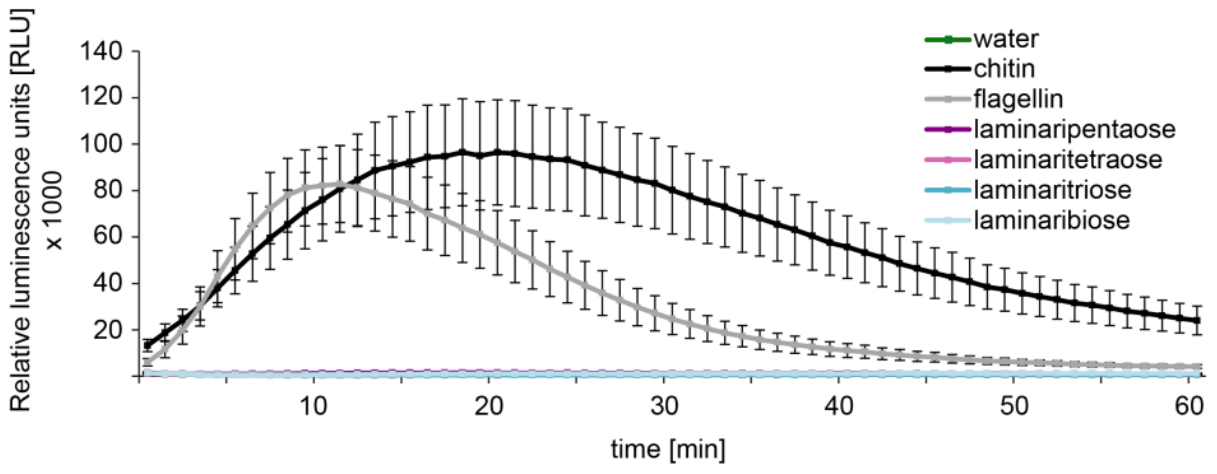

C

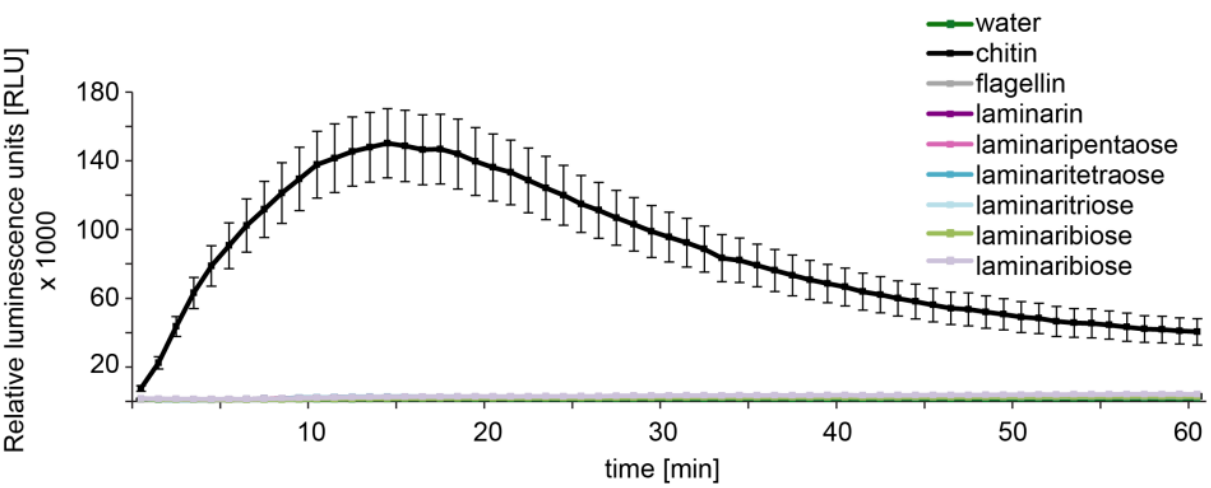

$\mathrm{D}$

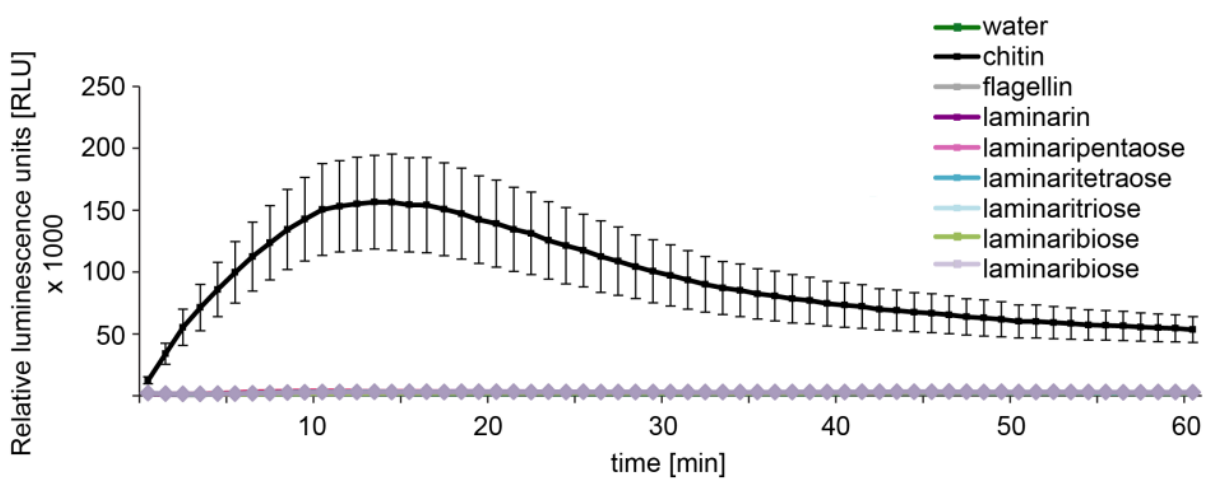

Figure S14. Generation of ROS in Arabidopsis ecotypes upon $\beta-1,3-$ glucan oligosaccharide treatment. Leaf discs of 5-7 week old Arabidopsis Col-0 (A and B), Ws-0 (C) or Ws-4 (D) plants were treated with water, $100 \mu \mathrm{g} \mathrm{ml}^{-1}$ chitin, $100 \mathrm{nM}^{\prime}$ flg22, $100 \mathrm{mg} \mathrm{ml}^{-1}$ laminarin or $100 \mathrm{\mu g} \mathrm{ml}^{-1} \beta$-1,3-glucan oligosaccharides of various lengths. Relative luminescence units (RLU) were recorded directly upon treatment every minute for $60 \mathrm{~min}$. The shown data represent the mean of eight leaf discs with SEM. The experiment was performed once. 


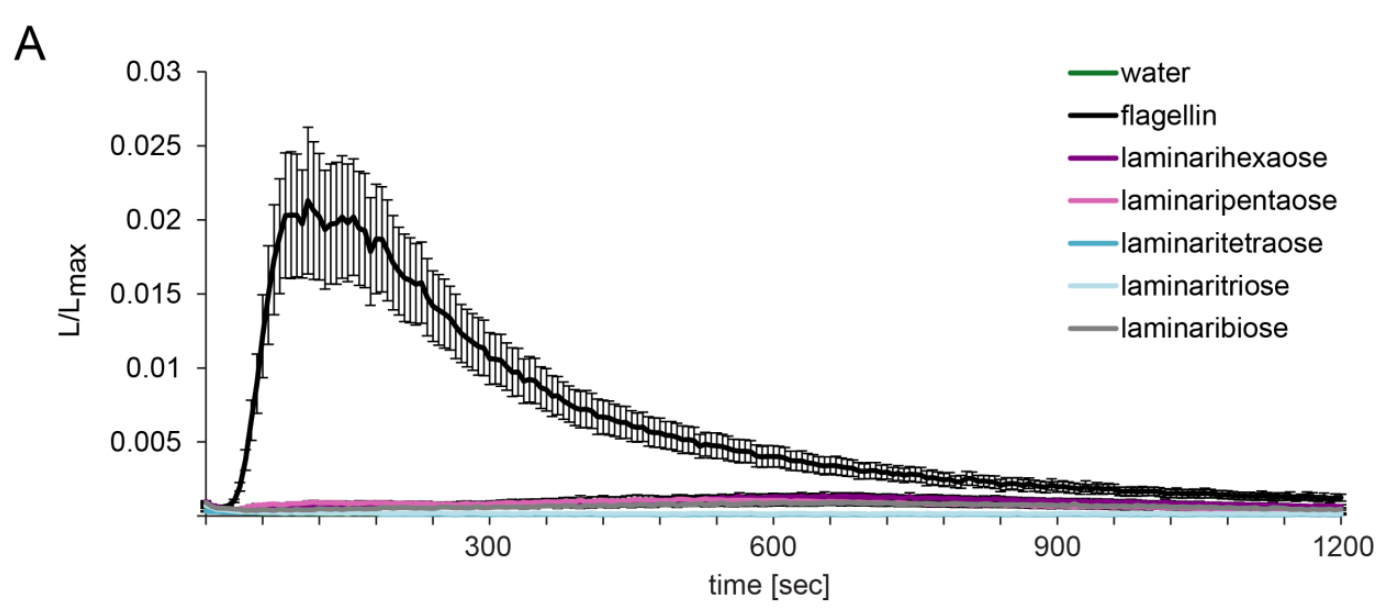

B
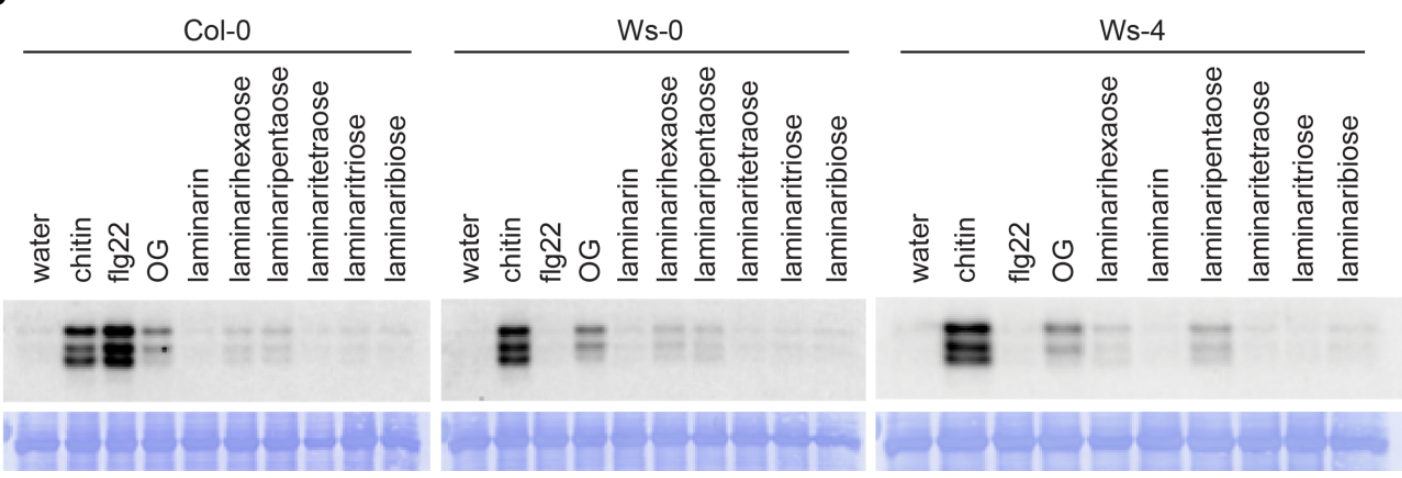

Figure S15. Activation of defence responses in Arabidopsis upon treatment with $\beta-1,3-g l u c a n s$. (A) Elevation of intracellular $\mathrm{Ca}^{2+}$. 8-10 day old Arabidopsis seedlings expressing the $\mathrm{Ca}^{2+}$ sensor protein aequorin were treated with water, $100 \mathrm{nM}$ flg22 or $100 \mu \mathrm{g} \mathrm{ml}^{-1}$ of the respective $\beta-1,3$-glucans. Elevation of $\mathrm{Ca}^{2+}$ was measured for $1200 \mathrm{sec}$ every $6 \mathrm{sec}(\mathrm{L})$. To obtain the total remaining luminescence $\left(\mathrm{L}_{\max }\right)$, the remaining aequorin was discharged by adding $\mathrm{CaCl}_{2}$ to each well and luminescence was recorded for 3 min in $6 \mathrm{sec}$ intervals. For normalization, the elicitor induced luminescence per $6 \mathrm{sec}(\mathrm{L})$ was divided by $L_{\max }$. The data shown represents the mean of 12 seedlings and error bars represent SEM. The experiment was performed once. (B) MAPK activation upon $\beta$-1,3-glucan oligosaccharide treatment. 14-day old in-vitro grown Arabidopsis Ws-0 and Ws-4 seedlings were treated with water, $10 \mu \mathrm{g} \mathrm{ml}^{-1}$ chitin, $50 \mathrm{nM}$ flg22 or $10 \mu \mathrm{g} \mathrm{ml}^{-1} \beta-1,3$-glucans of varying length for $12 \mathrm{~min}$. Activation of MAPK was analysed via immunoblot analyses using the p44/42-antibody. Lower panel shows Coomassie Brilliant Blue (CBB) staining as loading control. The experiment was performed three times with similar results. 


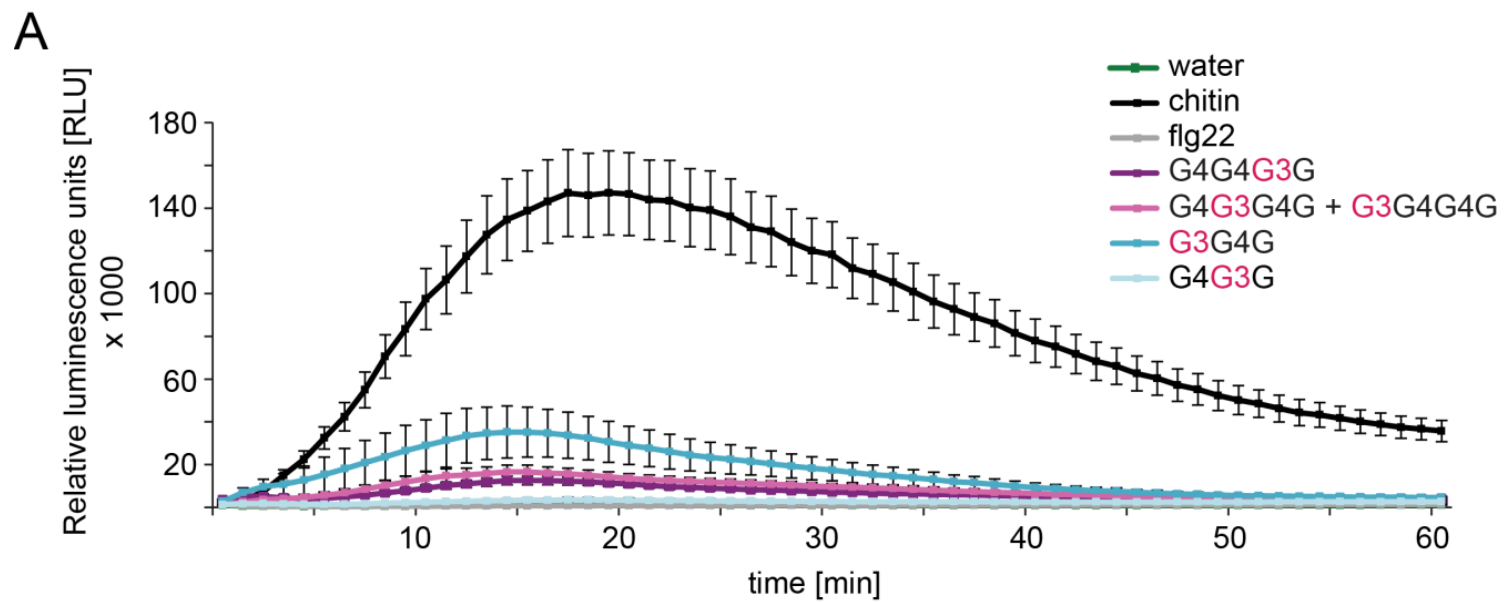

B

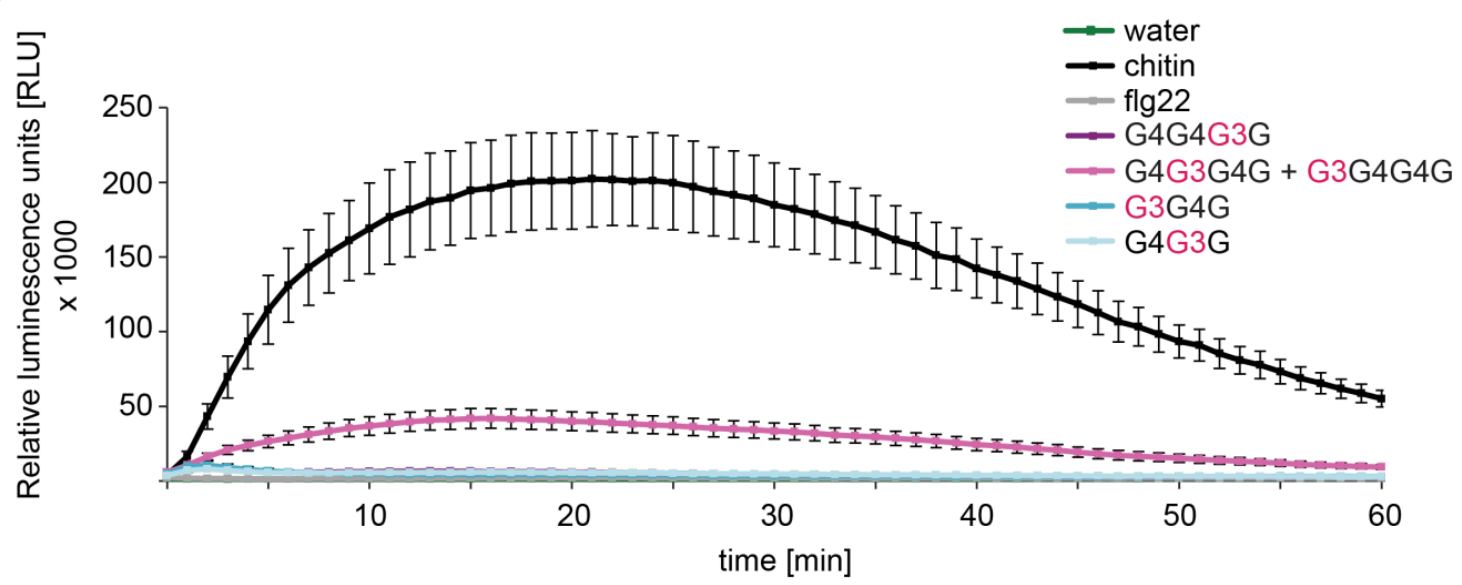

C

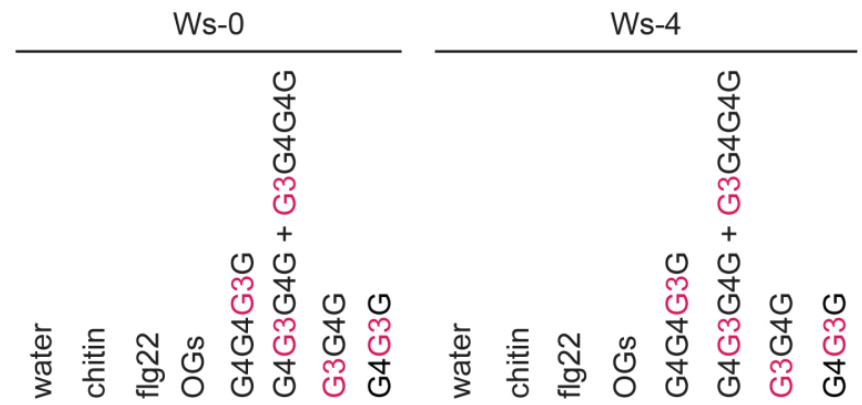

Figure S16. Generation of ROS and activation of MAPK in Ws-0 and Ws-4 upon elicitation with MLGs from Megazyme. (A) and (B). Generation of ROS. Leaf discs of 5-7 week old Arabidopsis Ws-0 (A) or Ws-4 (B) plants were treated with water, $100 \mathrm{\mu g} \mathrm{ml}^{-1}$ chitin, $100 \mathrm{nM}$ flg22 or $100 \mathrm{\mu g} \mathrm{ml}^{-1} \mathrm{MLG}$ oligomers. Relative light units (RLU) were recorded every minute for $60 \mathrm{~min}$. The shown data represent the mean of eight leaf discs with SEM. The experiment repeated two times with similar results. (C) MAPK activation upon MLG treatment. 14-day old in-vitro grown Arabidopsis Ws-0 and Ws-4 seedlings were treated with water, $10 \mu \mathrm{g} \mathrm{ml}^{-1}$ chitin, $50 \mathrm{nM}$ flg22 or $10 \mathrm{\mu g} \mathrm{ml}^{-1}$ MLG oligomers for $12 \mathrm{~min}$. Activation of MAPK was analysed via immunoblot analyses using the p44/42antibody. Lower panel shows Coomassie Brilliant Blue (CBB) staining as loading control. The experiment was repeated four times with similar results. 


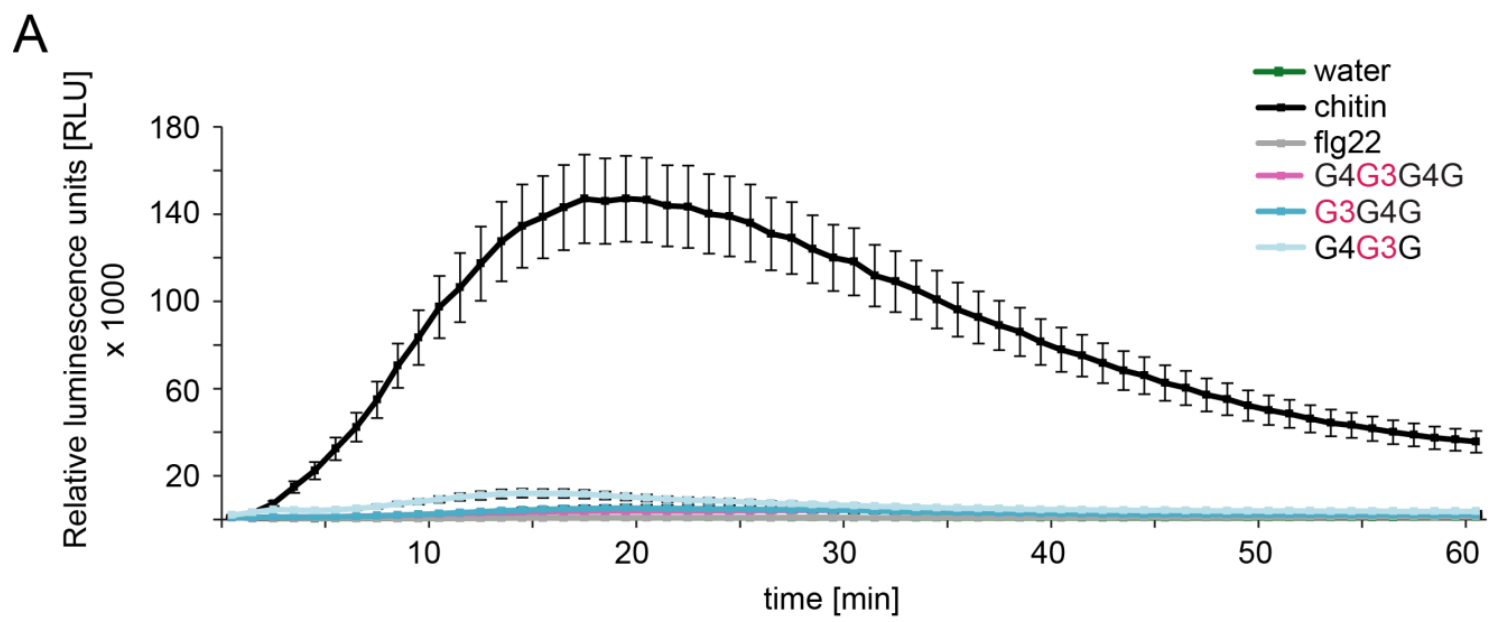

B

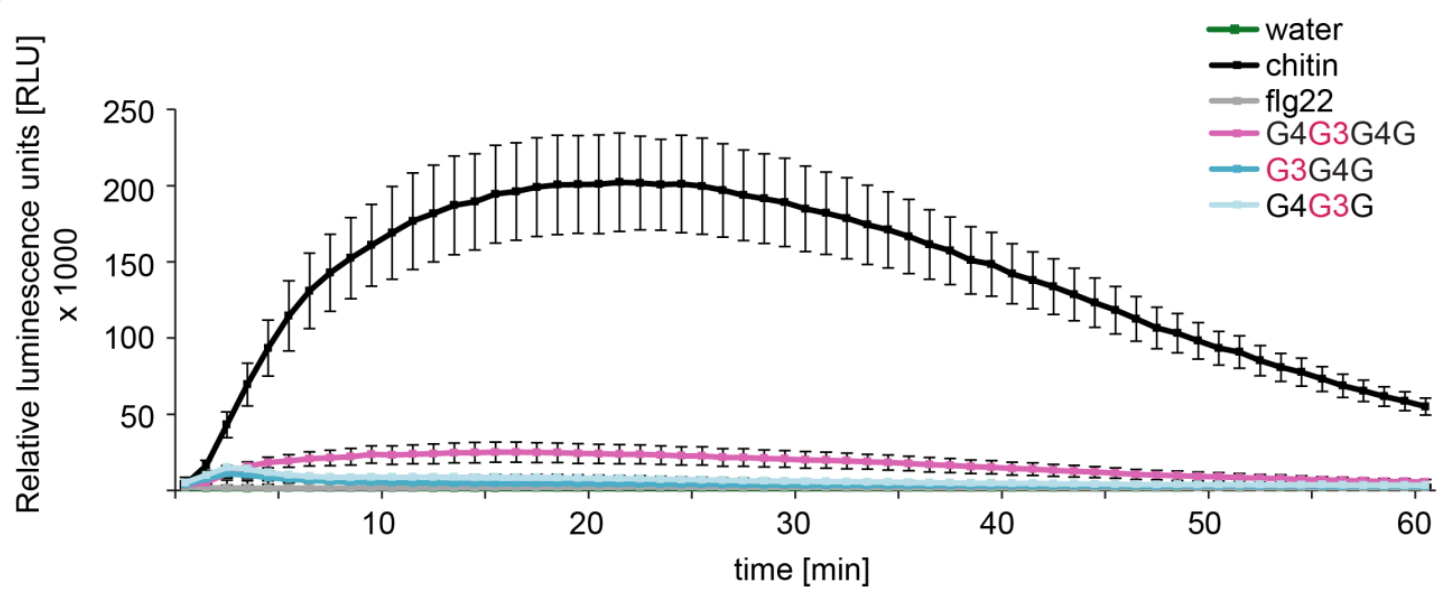

C
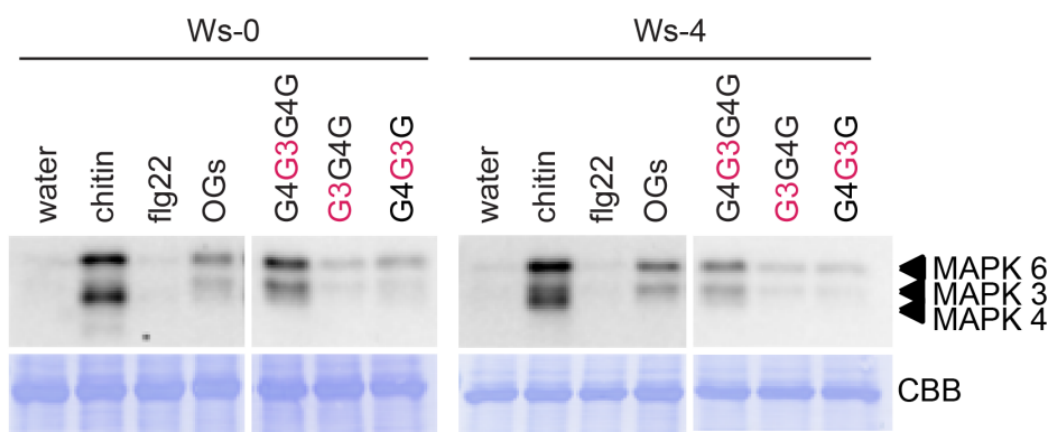

Figure S17. Activation of immune responses in Ws-0 and Ws-4 upon MLG oligomer treatment from Carbosynth. (A) and (B) Generation of ROS. Leaf discs of 5-7 week old Arabidopsis Ws-0 (A) or Ws-4 (B) plants were treated with water, $100 \mathrm{~g} \mathrm{ml}^{-1}$ chitin, $100 \mathrm{nM}$ flg22 or $100 \mathrm{\mu g} \mathrm{ml}^{-1} \mathrm{MLG}$ oligomers. Relative light units were recorded every minute for $60 \mathrm{~min}$. The shown data represent the mean of eight leaf discs with SEM. The experiment was repeated two times with similar results. (C) MAPK activation upon MLG treatment. 14-day old in-vitro grown Arabidopsis Ws- 0 and Ws-4 seedlings were treated with water, $10 \mu \mathrm{g} \mathrm{ml}^{-1}$ chitin, $50 \mathrm{nM}$ flg22 or $10 \mu \mathrm{g} \mathrm{ml} l^{-1} \mathrm{MLG}$ oligomers for $12 \mathrm{~min}$. Activation of MAPK was analysed via immunoblot analyses using the p44/42-antibody. Lower panel shows Coomassie Brilliant Blue (CBB) staining as loading control. The experiment was repeated four times with similar results. 

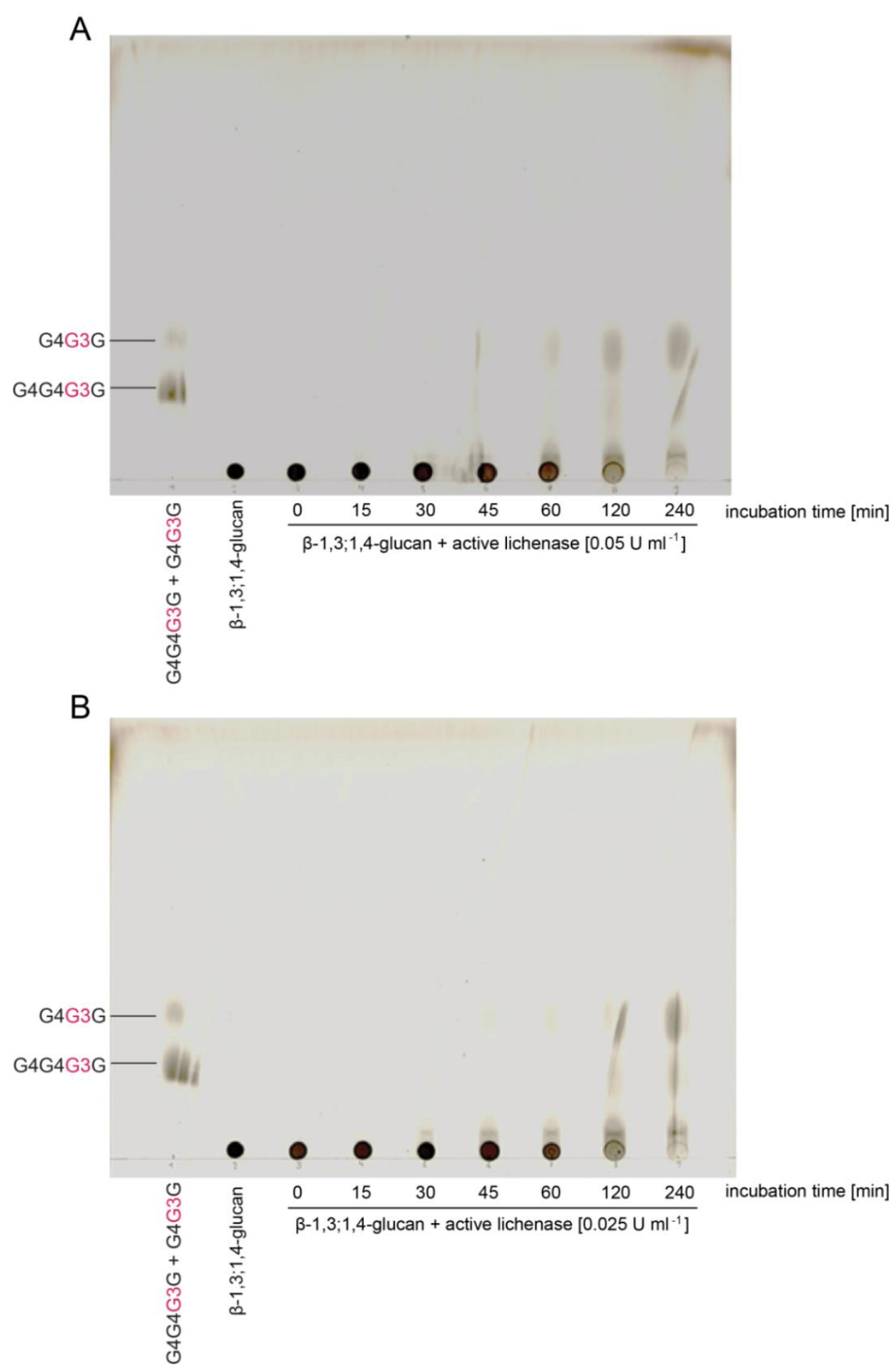

Figure S18. TLC of $\beta-1,3 ; 1,4-g l u c a n$ polymer hydrolysis. $10 \mathrm{mg} / \mathrm{ml}$ barley $\beta-1,3 ; 1,4-$ glucan polymer was dissolved in $100 \mathrm{mM}$ Sodium Phosphate buffer $(\mathrm{pH}=6.5)$ and incubated with either $(A) 0.05 \mathrm{U} \mathrm{ml}^{-1}$ lichenase or (B) $0.025 \mathrm{U} \mathrm{ml}^{-1}$ lichenase of $B$. subtilis. Upon $0,15,30,45,60,120$ and 240 min samples were taken and the enzyme was inactivated by incubating the hydrolysate for $15 \mathrm{~min}$ in boiling water. $10 \mu \mathrm{l}$ of the standards G4G4G3G + G4G3G (10 $\left.\mathrm{mg} \mathrm{ml}^{-1}\right)$, the $\beta-1,3 ; 1,4$-glucan polymer dissolved $100 \mathrm{mM}$ Sodium phosphate buffer $\left(10 \mathrm{mg} \mathrm{ml}^{-1}\right)$ or the respective hydrolysates was dropped onto the plate. Upon drying of the plate, it was put into a TLC running chamber containing TLC running buffer. The plate was developed by wetting it with $10 \%$ sulfuric acid in methanol and incubating on a heat plate at $99^{\circ} \mathrm{C}$ for $30-60 \mathrm{~min}$. 


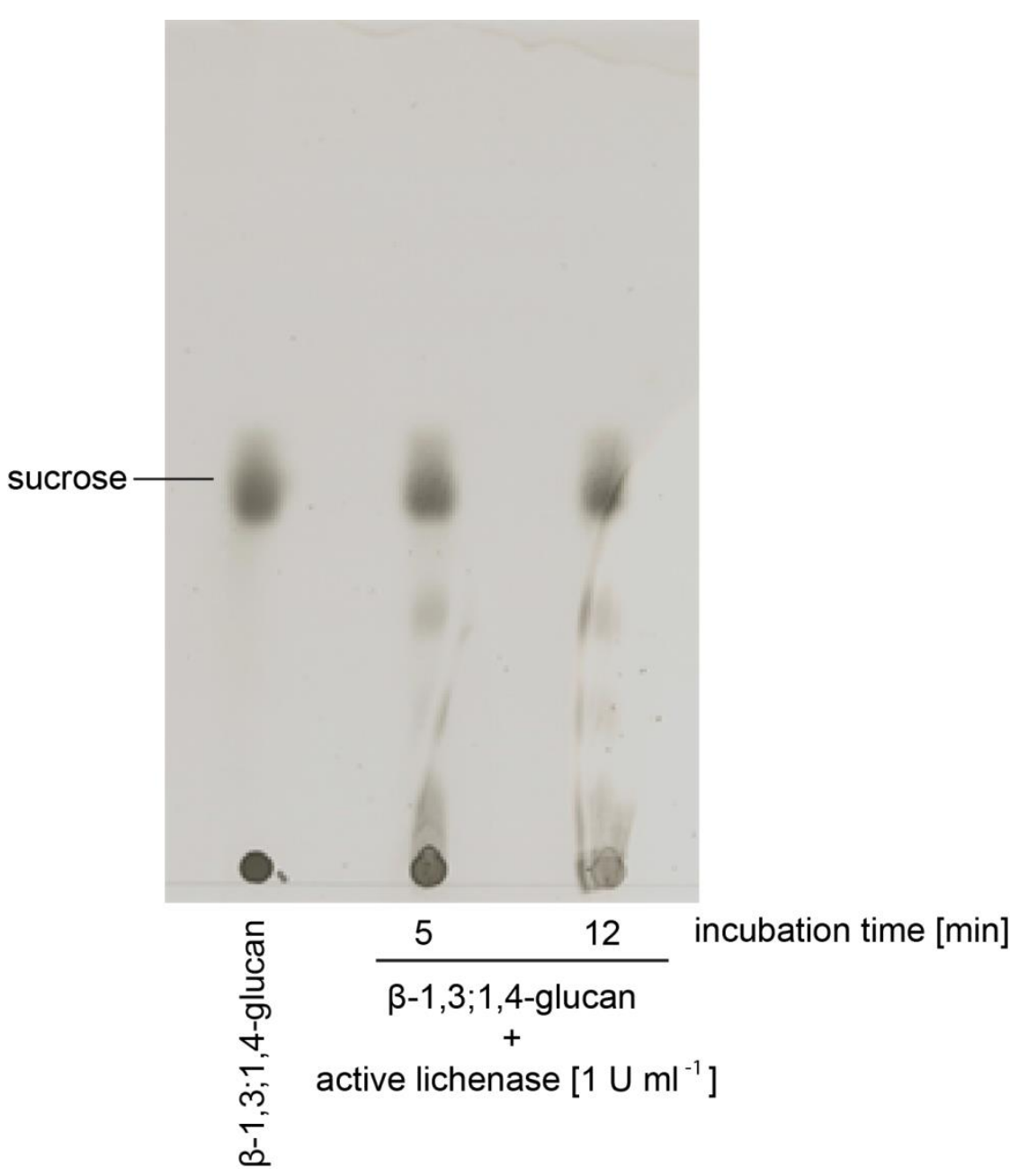

Figure S19. TLC of $\beta-1,3 ; 1,4-$ glucan polymer hydrolysis in $1 / 2 \mathrm{MS}+$ sucrose medium. $10 \mathrm{mg} / \mathrm{ml}$ barley $\beta-1,3 ; 1,4$-glucan polymer was dissolved in $1 / 2 \mathrm{MS}+$ sucrose medium and incubated with $1 \mathrm{U} \mathrm{ml}^{-1}$ lichenase of $B$. subtilis. Samples were taken upon 5 or $15 \mathrm{~min}$ and the enzyme was inactivated by incubating the hydrolysate for $15 \mathrm{~min}$ in boiling water. $10 \mu \mathrm{l} \beta-1,3 ; 1,4$-glucan polymer dissolved $1 / 2 \mathrm{MS}+$ sucrose medium (10 mg ml-1) or the respective hydrolysates was dropped onto the plate. Upon drying of the plate, it was put into a TLC running chamber containing TLC running buffer. The plate was developed by wetting it with $10 \%$ sulfuric acid in methanol and incubating on a heat plate at $99^{\circ} \mathrm{C}$ for $30-60 \mathrm{~min}$. 


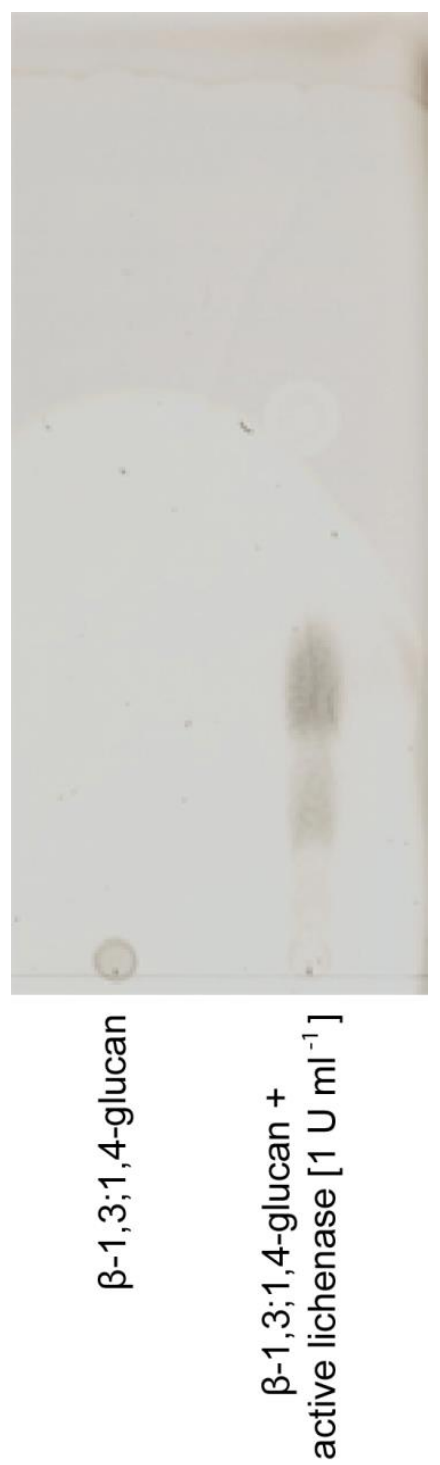

Figure S20. TLC of $\beta-1,3 ; 1,4-g l u c a n$ polymer hydrolysis for the forward and genetic screen. $10 \mathrm{mg} / \mathrm{ml}$ barley $\beta-1,3 ; 1,4$-glucan polymer was dissolved in $100 \mathrm{mM}$ Sodium phosphate buffer $(\mathrm{pH}=6.5)$ and incubated with $1 \mathrm{U} \mathrm{ml}^{-1}$ lichenase of $B$. subtilis. Upon $1 \mathrm{~h}$ incubation time, the enzyme was inactivated by incubating the hydrolysate for $15 \mathrm{~min}$ in boiling water. $10 \mu \mathrm{l}$-1,3;1,4-glucan polymer dissolved in $100 \mathrm{mM}$ Sodium phosphate buffer $\left(1 \mathrm{mg} \mathrm{ml}^{-1}\right)$ and the hydrolysate was dropped onto the plate. Upon drying of the plate, the plate was put into a TLC running chamber containing TLC running buffer. The plate was developed by wetting it with $10 \%$ sulfuric acid in methanol and incubating on a heat plate at $99^{\circ} \mathrm{C}$ for $30-60 \mathrm{~min}$. 

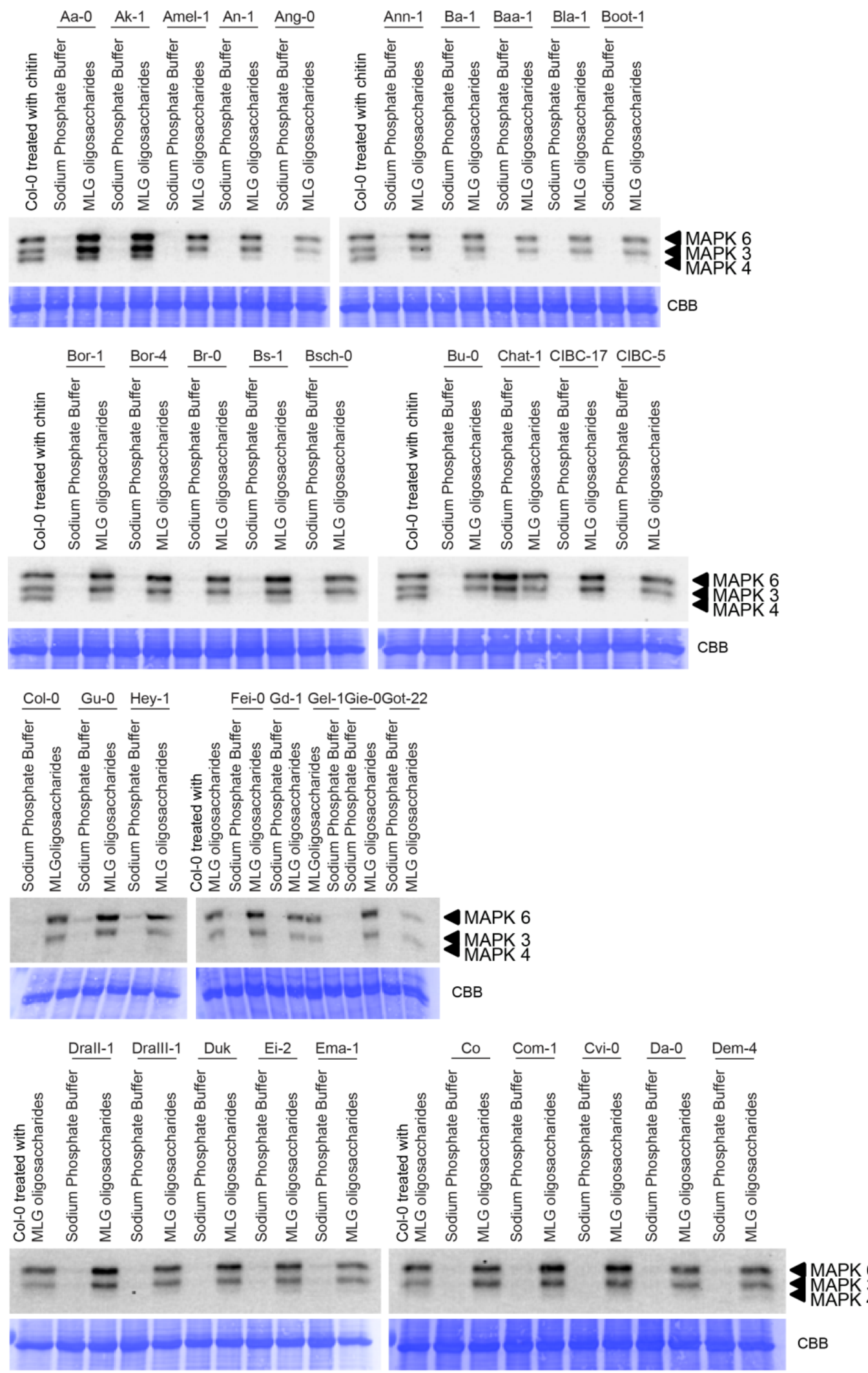

Figure S21. MAPK activation upon MLG treatment in different Arabidopsis ecotypes. 14-day old in-vitro grown Arabidopsis seedlings of various accessions were treated with $10 \mathrm{mM}$ Sodium Phosphate buffer or a 1:10 dilution of MLG oligosaccharides for $12 \mathrm{~min}$. Activation of MAPK6, MAPK3 and MAPK4 was analysed via Western Blot with the p44/42-antibody. Lower panel shows Coomassie Brilliant Blue (CBB) staining as loading control. The experiment was performed once. 

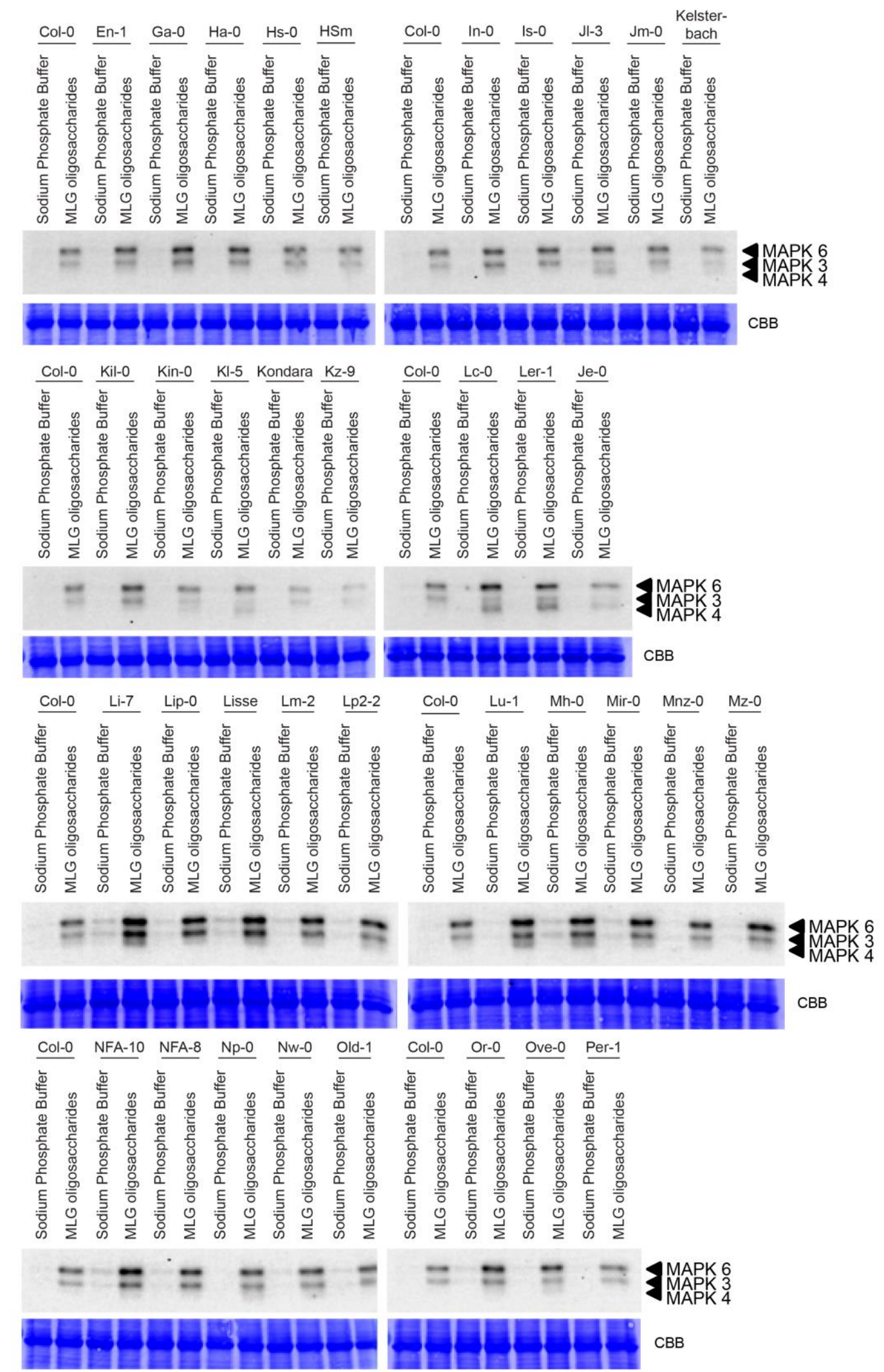

Figure S22. MAPK activation upon MLG treatment in various Arabidopsis accessions. 14-day old in-vitro grown Arabidopsis seedlings of various accessions were treated with $10 \mathrm{mM}$ Sodium Phosphate buffer or a 1:10 dilution of MLG oligosaccharides for $12 \mathrm{~min}$. Activation of MAPK6, MAPK3 and MAPK4 was analysed via Western Blot with the p44/42-antibody. Lower panel shows Coomassie Brilliant Blue (CBB) staining as loading control. The experiment was performed once. 


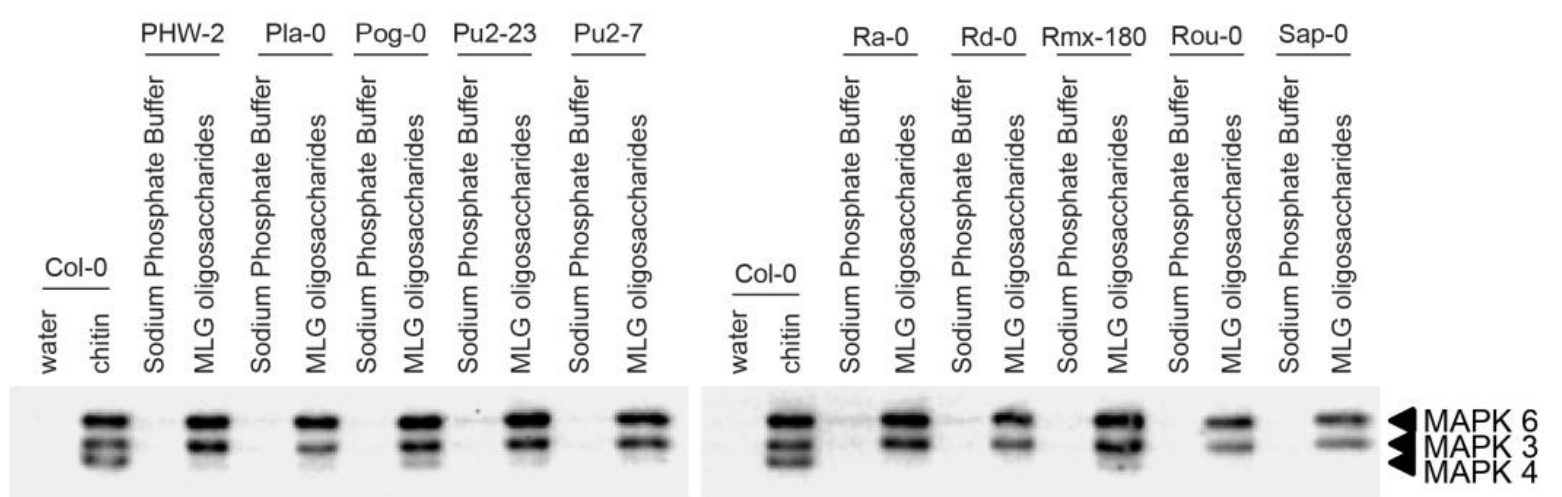

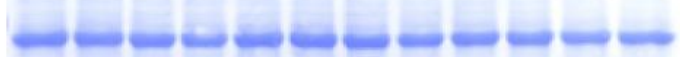

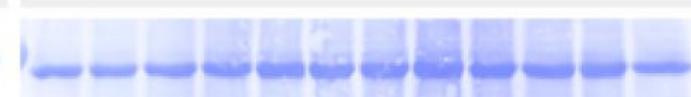

CBB

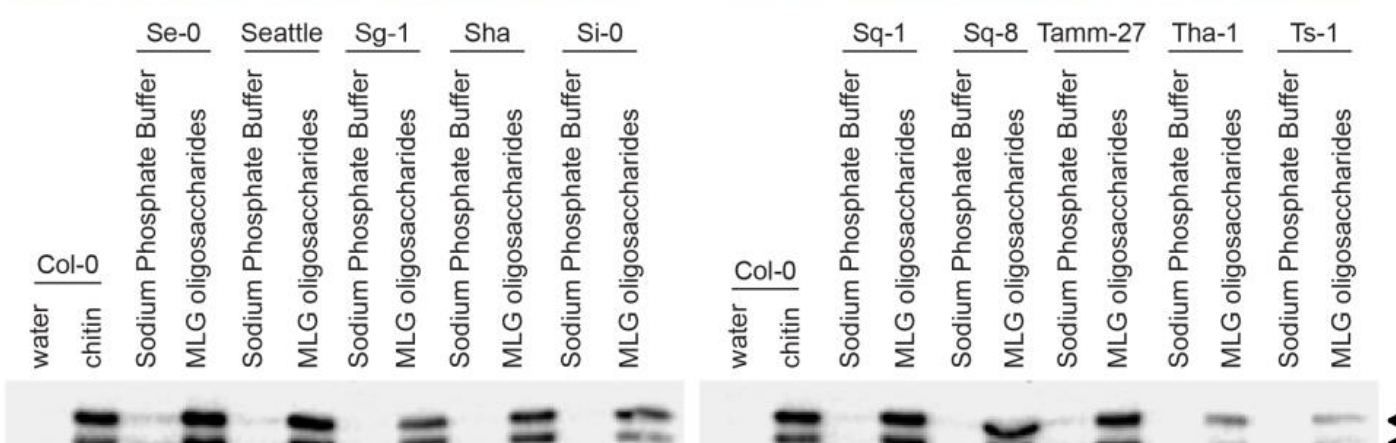

J脑A
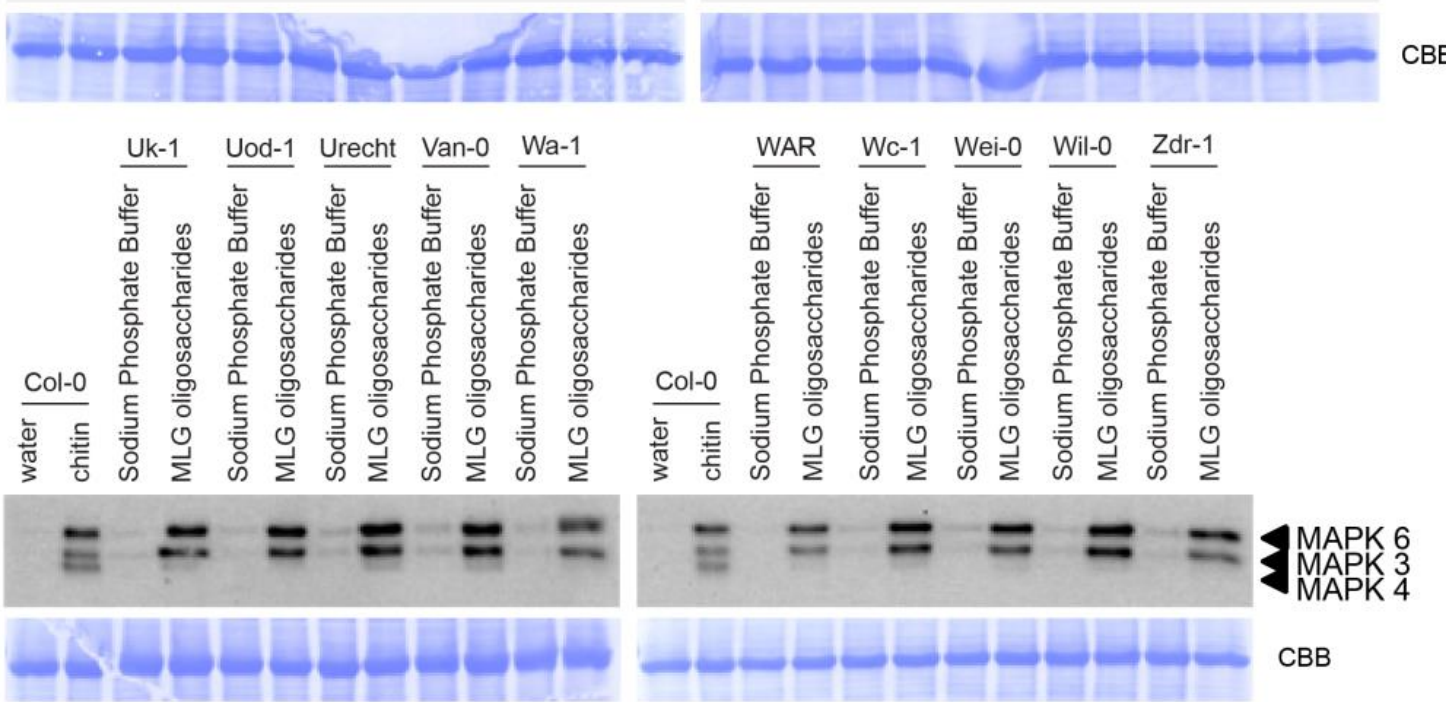

CBB

Figure S23. MAPK activation upon MLG treatment in various Arabidopsis ecotypes. 14-day old in-vitro grown Arabidopsis seedlings of various accessions were treated with $10 \mathrm{mM}$ Sodium Phosphate buffer or a 1:10 dilution of MLG oligosaccharides for 12 min. Activation of MAPK6, MAPK3 and MAPK4 was analysed via Western Blot with the p44/42-antibody. Lower panel shows Coomassie Brilliant Blue (CBB) staining as loading control. The experiment was performed once. 


\section{List of figures}

Figure 1. Schematic overview of the two-layered plant immune system ........................................... 2

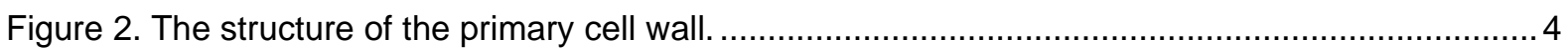

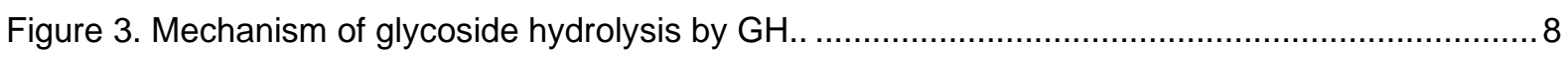

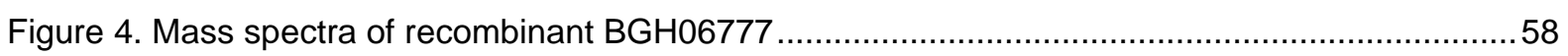

Figure 5. HPAEC-PAD chromatograms of $\beta$-1,3-glucan oligosaccharide hydrolysis by BGH06777 ...60

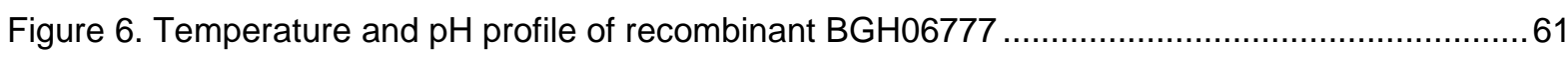

Figure 7. Michaelis-Menten model for an enzymatic reaction and the resulting equation....................62

Figure 8. Michaelis Menten plots of BGH06777 acting on $\beta$-1,3-oligosaccharides .............................63

Figure 9. Mass spectrometric analysis of the hydrolysis of laminarihexaose in presence of $\mathrm{H}_{2}{ }^{18} \mathrm{O}$ by

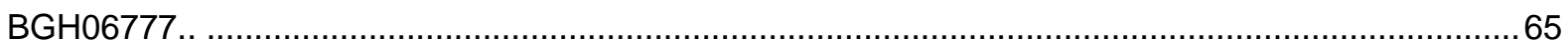

Figure 10. Activation of immune responses upon treatment with MLG oligomers in barley ................68

Figure 11. Activation of immune responses in Arabidopsis Col-0 by MLG oligomers from Megazyme..

Figure 12. Defence gene expression upon Megazyme MLG oligomer treatment in Arabidopsis Col-0

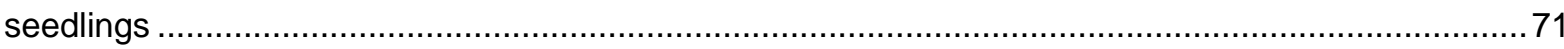

Figure 13. ROS burst generation and activation of MAPK in barley by MLG oligomers from Carbosynth.

Figure 14. Activation of immune responses in Arabidopsis Col-0 upon treatment with MLG oligomers

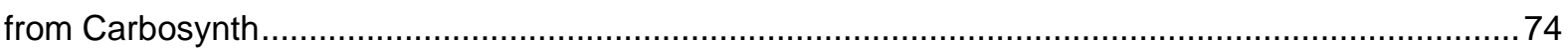

Figure 15. Expression of defence genes upon treatment with MLG oligomers from Carbosynth. .......75

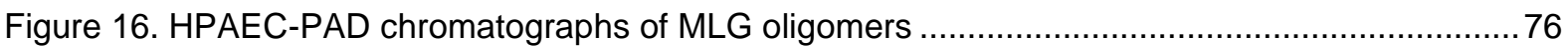

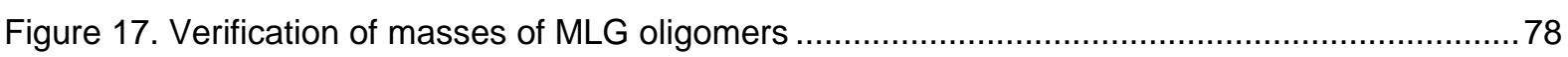

Figure 18. Schematic representation of a $\beta-1,3 ; 1,4$-glucan polymer and the obtained oligosaccharides upon treatment with $B$. subtilis lichenase

Figure 19. Calcium influx in Arabidopsis Col-0 upon treatment with MLG oligosaccharides ...............81 81

Figure 20. Activation of MAPK in Arabidopsis Col-0 upon treatment with MLG oligosaccharides ........82

Figure 21. Defence gene expression in Arabidopsis Col-0 upon treatment with MLG oligosaccharides. .83

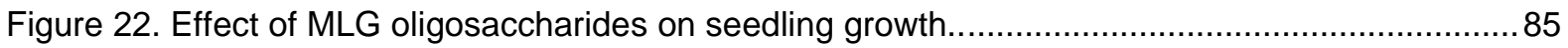

Figure 23. Activation of MAPK6 and MAPK3 in different LysM-RLKs and LysM-RLPs mutants upon MLG oligosaccharide treatment

Figure 24. Activation of MAPK6 and MAPK3 in fls2c and efr-1 upon MLG oligosaccharide treatment.

Figure 25. Activation of MAPK6 and MAPK3 in different co-receptor mutants upon MLG

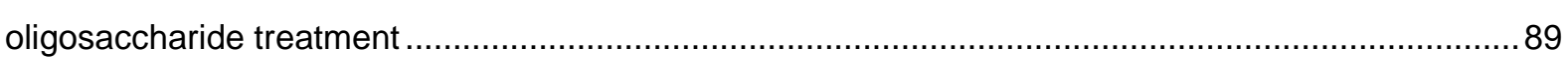

Figure 26. MAPK activation in the parental ecotypes of the MAGIC lines .........................................91 


\section{List of tables}

Table 1. List of T-DNA insertion and transgenic lines used in this work..........................................15

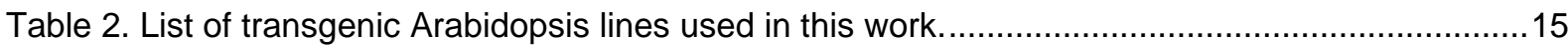

Table 3. List of Arabidopsis thaliana accessions used in this work. ................................................ 16

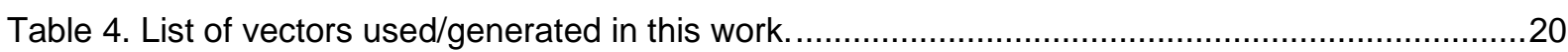

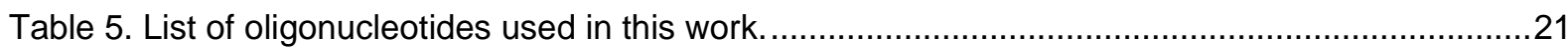

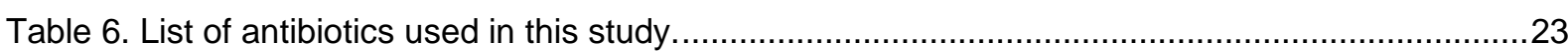

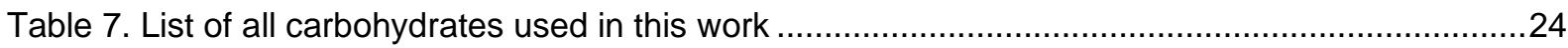

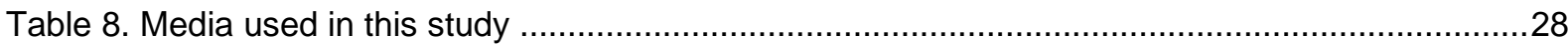

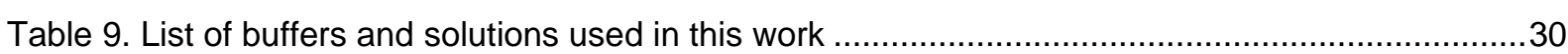

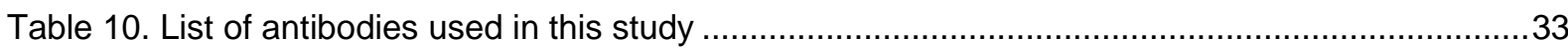

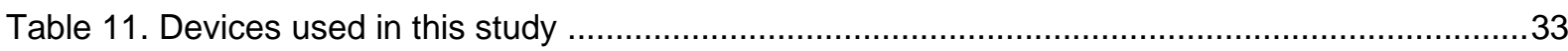

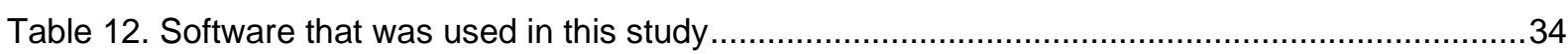

Table 13. General temperature profile for PCR with Q5 High Fidelity Polymerase.............................40

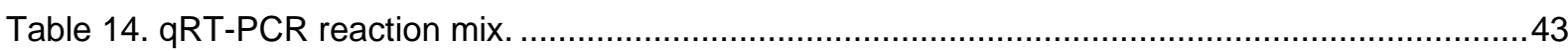

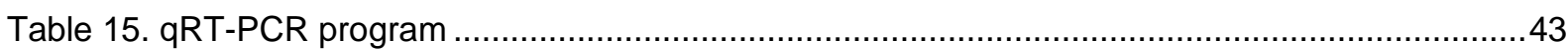

Table 16. Composition of SDS PAGE Gel buffers and mixes used in this study .................................46

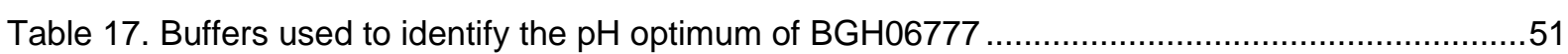

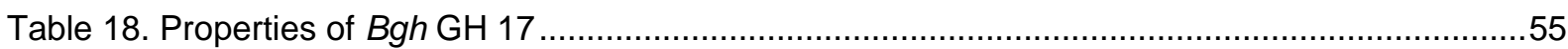

Table 19. Michaelis-Menten parameters for BGH06777 on $\beta$-1,3-oligosaccharides ...........................63

Table 20. Degree of ${ }^{18} \mathrm{O}$ labelled laminaripentaose (G3G3G3G) and laminaribiose (G3G) derived from hydrolysis of laminarihexaose (G3G3G3G3G3G) by BGH06777. 


\section{List of supplemental tables and figures}

Table S1. Predicted motifs of GH in Bgh. 126

Table S2. In planta RPM values of $B g h$ GH families 5, 16, 17, 47 and 76 upon 6, 12, 18 and 24 hpi.. 130

Figure $\mathrm{S} 1$. Small scale expression of $\mathrm{Bgh} \mathrm{GH} 17$ genes 132

Figure S2. SDS PAGE of recombinant BGH0677 133

Figure S3. Influx of $\mathrm{Ca}^{2+}$ in Col-0 upon oligo- and polysaccharide treatment 134

Figure S4. Generation of ROS in Col-0 upon oligo- and polysaccharide treatment...... 135

Figure S5. Generation of ROS in Ws-0 upon oligo- and polysaccharide treatment 136

Figure S6. Generation of ROS in Ws-4 upon oligo- and polysaccharide treatment..... 137

Figure S7. MAPK activation in Col-0 upon treatment with several oligo- and polysaccharides ..........138

Figure S8. MAPK activation in Ws-0 upon treatment with several oligo- and polysaccharides ..........139

Figure S9. MAPK activation in Ws-4 upon treatment with several oligo- and polysaccharides ..........140

Figure S10. ROS burst generation in three Arabidopsis ecotypes upon cellohexaose treatment.......141

Figure S11. Activation of immune responses upon cellohexaose elicitation ....................................142

Figure S12. ROS burst generation upon xylohexaose treatment in different Arabidopsis ecotypes ...143

Figure $\mathrm{S} 13 . \mathrm{Ca}^{2+}$ influx and MAPK activation upon xylohexaose elicitation

Figure S14. Generation of ROS in Arabidopsis ecotypes upon $\beta-1,3$-glucan oligosaccharide

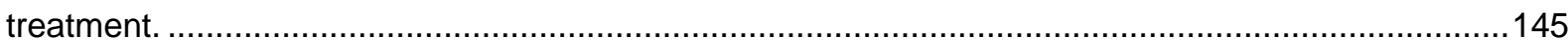

Figure S15. Activation of defence responses in Arabidopsis upon treatment with $\beta$-1,3-glucans.......146 Figure S16. Generation of ROS and activation of MAPK in Ws-0 and Ws-4 upon elicitation with MLGs from Megazyme

Figure S17. Activation of immune responses in Ws-0 and Ws-4 upon MLG oligomer treatment from

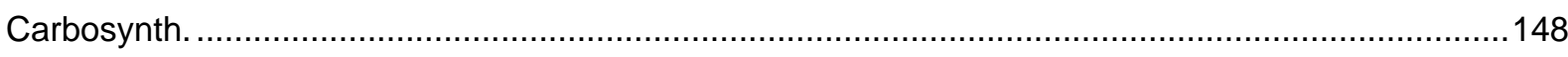

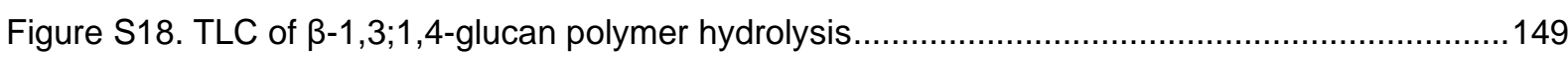

Figure S19. TLC of $\beta-1,3 ; 1,4$-glucan polymer hydrolysis in $1 / 2 \mathrm{MS}+$ sucrose medium ......................150

Figure S20. TLC of $\beta$-1,3;1,4-glucan polymer hydrolysis for the forward and genetic screen.............151

Figure S21. MAPK activation upon MLG treatment in different Arabidopsis ecotypes ......................152

Figure S22. MAPK activation upon MLG treatment in various Arabidopsis accessions ....................153

Figure S23. MAPK activation upon MLG treatment in various Arabidopsis ecotypes ........................154 


\section{Danksagung}

An dieser Stelle möchte ich mich bei Allen bedanken, die mich während meiner Promotion unterstützt und begleitet haben - sei es privat oder akademisch.

Zuerst möchte ich mich bei Prof. Dr. Volker Lipka dafür bedanken, dass er mir die Möglichkeit gegeben hat in seiner Abteilung an einem interessanten Projekt $z u$ arbeiten, das molekularbiologische aber auch biochemische Aspekte beinhaltete. Außerdem möchte ich mich für die vielen Diskussionen und die konstruktive Kritik während Meetings oder auch zwischendurch bedanken, die sehr zum Erfolg des Projektes beigetragen haben. Seine Begeisterung während dieser Diskussionen haben mich auch dazu angeregt eigene Ideen zu entwickeln und stets motiviert zu bleiben. Danke außerdem für die wertvolle und uneingeschränkte Unterstützung und das Vertrauen, das ich nicht nur während der Promotion erfahren habe, sondern auch während des gesamten Bachelor- und Masterstudiums. Seine Tür stand jederzeit offen und er hatte immer ein offenes Ohr für kleine und große Probleme oder Anliegen des Alltags oder im Labor. Außerdem war er auch immer für einen $S p a ß z u$ haben. Seine Art hat eine familiäre Atmosphäre in der Abteilung geschaffen und die Produktivität gefördert. Weiterhin möchte ich mich herzlich dafür bedanken, dass er mich immer gefördert und gefordert hat. Ich danke ihm außerdem für seine Funktion als Erstprüfer und Doktorvater sowie für die Begutachtung meiner Arbeit.

Ein großes Dankeschön geht auch an PD Dr. Till Ischebeck für die Übernahme des Koreferats. Außerdem möchte ich mich für die Bereitstellung der großen Kollektion an Arabidopsis Ökotypen bedanken ohne die der Screen nicht möglich gewesen wäre.

A great thanks goes also to Prof. Dr. Harry Brumer. He welcomed me warmly in Vancouver and in his lab so that it directly felt like home. His knowledge in enzymology was a great support in the promotion of this project and contributed to the success of this project. During my two stays but also during skype meetings, I benefitted greatly from his experience and learned a lot about carbohydrate enzymology. Thank you also for your patience in proof-reading this work and your constructive comments and suggestions.

Thanks also to both, PD Dr. Till Ischebeck and Prof. Dr. Harry Brumer for being part of my examination board and for your great suggestions and ideas during yearly discussions after the progress reports.

Bei Prof. Dr. Ivo Feußner, Prof. Dr. Gerhard Braus und Dr. Marcel Wiermer möchte ich mich bedanken, dass sie zusammen mit meinem Betreuungsausschuss meine Prüfungskomission bilden. 
Vielen Dank auch an Dr. Elena Petutschnig für die Betreuung während der Arbeit. Sie war eine große Hilfe bei der Durchführung vieler Experimente und hat mit vielen Ratschlägen und Vorschlägen das Projekt weiterentwickelt.

Des Weiteren möchte ich mich bei PD Dr. Thomas Teichmann und Dr. Hassan Ghareeb für die Ratschläge und Anregungen während der 2-wöchentlichen „Chitin-Meetings“ bedanken sowie für die Hillfe bei kleinen und großen Problemen.

Ein riesen Dankeschön geht auch an unsere herzensguten technischen Assistenten Gaby, Sabine, Ludmilla und Melanie für die wertvolle Hilfe bei Experimenten, die Bestellung von Labormaterial, das Bereitstellen von Puffern und das Aufräumen von Laborplätzen, aber auch das offene Ohr bei Problemen abseits der Arbeit. Danke auch an unsere fleißigen Gärtnerinnen Feli und Susanne für die Hilfe bei den vielen Siebearbeiten und die vielen Gespräche, die sich nicht nur um die Arbeit drehten. Ohne euch würde der Alltag im Labor nicht so reibungslos ablaufen - danke dafür!

Weiterhin möchte ich mich bei meinen studentischen Hilfskräften Melina Schwier, Esther Schneider und Julia Lechtenberg bedanken, die viele Stunden an der Sterilbank verbracht und mir damit sehr viel Arbeit abgenommen haben.

Ein riesiges Dankeschön geht auch an meine lieben Doktorandenkollegen Leonie, Dimitri, Julia, Mohammed, Chrissi, Lena, Mo, Mascha, Daniel und Denise. Danke für die aufmunternden Worte, wenn es einmal nicht so lief wie geplant, Plaudereien, die sich nicht um Wissenschaft drehten, Albernheiten und die wertvollen Ratschläge für Experimente. Ein besonderer Dank geht dabei an Leonie und Denise. Die Beiden sind während der Promotion gute Freundinnen von mir geworden vielen Dank, dass ihr mir immer mit Rat und Tat zur Seite standet.

Außerdem möchte ich mich bei der ganzen Abteilung für die familiäre Arbeitsatmosphäre und die vielen Aktivitäten außerhalb des Labors, die mit Freude angegangen wurden, bedanken.

I would also like thank people outside my lab who helped me with this project. Dr. Yann Mathieu and Maria Cleveland taught me how to transform Pichia pastoris, screen for transformants and to purify a protein. Nicholas McGregor showed me the biochemical methods to functionally characterize a glycoside hydrolase. A great thanks goes also to Namrata Jain, Gregory Arnal and Kazune Tamura who helped me with the analysis and hydrolysis of carbohydrates. A special thanks to Hila Behar who helped me with general things and became a close friend - I miss you.

Furthermore I would like to take this opportunity and thank the whole Brumer lab for welcoming me so warmly during my two stays. Everyone was so friendly and helpful that Vancouver and the lab felt directly like home - thank you! 
Weiterhin möchte ich mich auch bei der unseren Kollaborationspartnern am IPK in Gatersleben für die Bereitstellung der Genomdaten von Bgh und den vorläufigen Ergebnissen der HIGS Analysen herzlich bedanken. Die Bereitstellung dieser Daten haben sehr zum Erfolg dieser Arbeit beigetragen.

Ein großes Dankeschön gebührt auch allen Mitgliedern des IRTG PRoTECTs. Danke für die schönen Retreats auf der Burg Ludwigstein mit Lagerfeuer und Musik und in Vancouver. Danke für die vielen Treffen in denen wissenschaftliche, aber auch private Anliegen besprochen wurden. Danke für das gemeinsame Lachen, Aufregen und die Unterstützung. Es wird mir fehlen! Ein besonderer Dank geht dabei an Hila, Kevin, Jessi, Mimi, Daniel, Dimi und Denise - ihr habt die Zeit in Kanada zu etwas noch Besonderem gemacht und unsere vielen Erlebnisse werde ich nicht vergessen. A big thanks also to Jelena and Aswin who had always time for a little talk and were helpful in all regards outside and inside the lab.

Von Herzen möchte ich mich auch bei Ronja bedanken, die seit der Bachelorarbeit nicht nur eine Kommolitonin, sondern auch eine sehr gute Freundin ist (Du, ich und das Agrobakterium!). Danke für deine wunderbare Freunschaft, dein offenes Ohr und die großartige Unterstütztung seit dem Bachelor. Du bist es einfach.

Ein herzliches Dankeschön geht auch an meine Freunde außerhalb des Labors: Melissa, Lisa, Neele und Finn. Danke für die Ablenkung, Aufmunterung und Unterstützung trotz der großen Entfernung in den letzten Jahren!

Nicht zuletzt möchte ich mich herzlich bei meiner Familie für die moralische Unterstützung während der letzten Jahre bedanken. Besonders danke ich meinem Papa, meinem Bruder und meiner Schwägerin, die immer an mich geglaubt haben und ohne die ich es nicht geschafft hätte - danke! 